\title{
Iris Recognition: Robust Processing, Synthesis, Performance Evaluation and Applications
}

Jinyu Zuo

West Virginia University

Follow this and additional works at: https://researchrepository.wvu.edu/etd

\section{Recommended Citation}

Zuo, Jinyu, "Iris Recognition: Robust Processing, Synthesis, Performance Evaluation and Applications" (2011). Graduate Theses, Dissertations, and Problem Reports. 3485.

https://researchrepository.wvu.edu/etd/3485

This Dissertation is protected by copyright and/or related rights. It has been brought to you by the The Research Repository @ WVU with permission from the rights-holder(s). You are free to use this Dissertation in any way that is permitted by the copyright and related rights legislation that applies to your use. For other uses you must obtain permission from the rights-holder(s) directly, unless additional rights are indicated by a Creative Commons license in the record and/ or on the work itself. This Dissertation has been accepted for inclusion in WVU Graduate Theses, Dissertations, and Problem Reports collection by an authorized administrator of The Research Repository @ WVU.

For more information, please contact researchrepository@mail.wvu.edu. 


\title{
Iris Recognition:
}

\section{Robust Processing, Synthesis, Performance Evaluation and Applications}

\author{
by \\ Jinyu Zuo \\ Dissertation submitted to the \\ College of Engineering and Mineral Resources \\ at West Virginia University \\ in partial fulfillment of the requirements \\ for the degree of \\ Doctor of Philosophy \\ in \\ Electrical Engineering \\ Natalia A. Schmid, Ph.D., Chair \\ Donald A. Adjeroh, Ph.D. \\ Lawrence A. Hornak, Ph.D. \\ Xin Li, Ph.D. \\ Cun-Quan Zhang, Ph.D. \\ Lane Department of Computer Science and Electrical Engineering \\ Morgantown, West Virginia \\ 2011
}

Keywords: Biometrics, iris recognition, pattern recognition, image quality

Copyright 2011 Jinyu Zuo 


\begin{abstract}
Iris Recognition:

Robust Processing, Synthesis, Performance Evaluation and Applications

by

Jinyu Zuo
\end{abstract}

The popularity of iris biometric has grown considerably over the past few years. It has resulted in the development of a large number of new iris processing and encoding algorithms. In this dissertation, we will discuss the following aspects of the iris recognition problem: iris image acquisition, iris quality, iris segmentation, iris encoding, performance enhancement and two novel applications.

The specific claimed novelties of this dissertation include: (1) a method to generate a large scale realistic database of iris images; (2) a crosspectral iris matching method for comparison of images in color range against images in Near-Infrared (NIR) range; (3) a method to evaluate iris image and video quality; (4) a robust quality-based iris segmentation method; (5) several approaches to enhance recognition performance and security of traditional iris encoding techniques; (6) a method to increase iris capture volume for acquisition of iris on the move from a distance and (7) a method to improve performance of biometric systems due to available soft data in the form of links and connections in a relevant social network. 


\section{Acknowledgements}

My deepest gratitude is to my committee chair and advisor, Dr. Natalia A. Schmid, for giving me the opportunity to work with her. I have been amazingly fortunate to have an advisor who patiently taught me how to do research and gave me enough freedom to explore on my own. This dissertation would not be possible without her constant guidance, encouragement and support. I am also thankful to her for encouraging the use of correct grammar and consistent notation in my writings, and for carefully reading and commenting on countless revisions of published papers and this manuscript.

I would also like to thank Dr. Donald A. Adjeroh, Dr. Lawrence A. Hornak, Dr. Xin Li and Dr. Cun-Quan Zhang for being on my committee.

I have been fortunate to have had the opportunity to take courses with Dr. Natalia A. Schmid, Dr. Xin Li, Dr. Matthew C. Valenti and Dr. Tim McGraw, and their teachings have been essential to my understanding of the subject.

I thank the Exploratory Computer Vision Group of the IBM Thomas J. Watson Research Center for the wonderful experience of being an intern. Especially, I thank Dr. Nalini K. Ratha and Dr. Jonathan H. Connell for numerous helpful advices and inspiring discussions.

Next, I would also like to thank the students in the Statistical Signal Processing research lab with whom I have had the pleasure of working alongside. In particular, I would like to thank my colleagues Xiaohan Chen, Nathan D. Kalka and Francesco Nicolo, who have been incredible friends to me over the years.

Finally, I would like to express my gratitude to my family, for keeping me going through all the stresses.

The research work presented in this dissertation was supported in part by Center for Identification Technology Research (CITeR), Office of Naval Research, U.S. Army Research Laboratory (ARL) and IBM Research. 


\section{Contents}

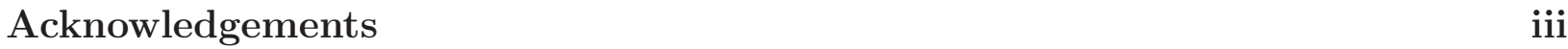

List of Figures $\quad$ vii

List of Tables $\quad$ xiv

1 Introduction 1

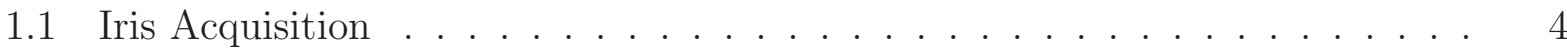

1.1.1 Successful Commercial Systems . . . . . . . . . . . . . . . 4

1.1 .2 Visible Light . . . . . . . . . . . . . . . . . . . . . . . . 4

1.1 .3 Long Distance . . . . . . . . . . . . . . . . . . . . . . . . 4

1.1.4 Generated Data . . . . . . . . . . . . . . . . . . . 5

1.2 Iris Quality . . . . . . . . . . . . . . . . . . . . 6

1.2.1 Selecting the Best Quality Iris Image . . . . . . . . . . . . 6

1.2 .2 Processing Iris Data . . . . . . . . . . . . . . . . . . 6

1.2.3 Adaptive Biometric Authentication . . . . . . . . . . . . . . . 6

1.3 Iris Segmentation . . . . . . . . . . . . . . . . . . . . 7

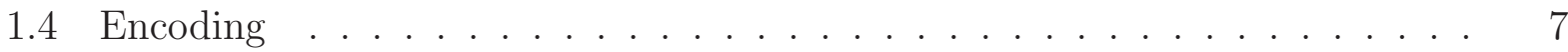

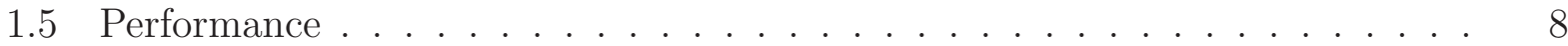

1.6 Applications . . . . . . . . . . . . . . . . . . . . . 9

1.7 Organization of this Dissertation $\ldots \ldots \ldots \ldots \ldots$

2 Synthetic Iris 11

2.1 Introduction . . . . . . . . . . . . . . . . . . . . . . . 11

2.2 Methodology . . . . . . . . . . . . . . . . . . . . 15

2.3 Performance Analysis: Image and Texture Level . . . . . . . . . . . . . . . . 22

2.3 .1 Visual Evaluation . . . . . . . . . . . . . . . . . . . . . . . . 22

2.3 .2 Texture Analysis . . . . . . . . . . . . . . . . . . . . . . 22

2.4 Performance Analysis: Decision Level . . . . . . . . . . . . . . . . . . . . 31

2.4.1 Verification Performance . . . . . . . . . . . . . . . . . . . . . 31

2.4.2 Analysis of Degrees of Freedom . . . . . . . . . . . . . . . . . 31

2.4.3 Performance Extrapolation . . . . . . . . . . . . . . . . . . . 34

2.5 Conclusion . . . . . . . . . . . . . . . . . . . . . . . . . . . . . . . . . . 39 
3 Multi-spectral Iris $\quad 41$

3.1 Introduction . . . . . . . . . . . . . . . . . . . . . . 41

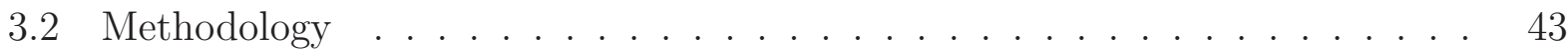

3.2.1 Multi-spectral Iris Dataset and Data used in Simulations . . . . . . . 43

3.2.2 Proposed Predictive Model . . . . . . . . . . . . . . . . . . . . . 44

3.2 .3 Neural Network . . . . . . . . . . . . . . . . . . . . . . . 45

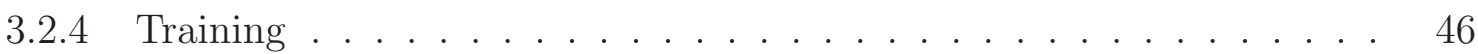

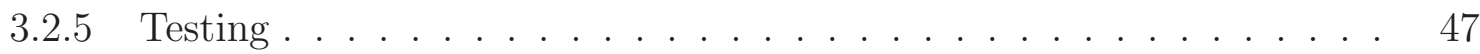

3.2.6 Model Selection Criterion . . . . . . . . . . . . . . . . . 47

3.2.7 Illustration of Predicted Results . . . . . . . . . . . . . . . . . . . . 48

3.3 Numerical Results . . . . . . . . . . . . . . . . . . . . . . . . . . . . . . . . . . . . . . . . . . . .

3.4 Conclusions and Future Work . . . . . . . . . . . . . . . . . . . 52

4 Iris Quality $\quad 55$

4.1 Introduction . . . . . . . . . . . . . . . . . . . . 55

4.2 Frame Selection . . . . . . . . . . . . . . . . . . . . 57

4.2 .1 MBGC Data . . . . . . . . . . . . . . . . . . . . . . . . . . . . . . .

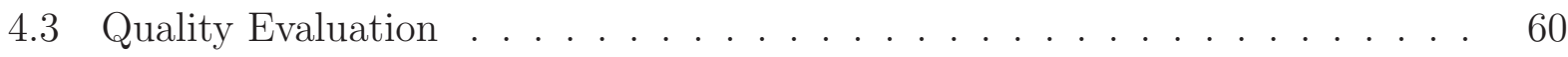

4.3.1 Segmentation Scores . . . . . . . . . . . . . . . 60

4.3 .2 Interlacing . . . . . . . . . . . . . . . . . . . 61

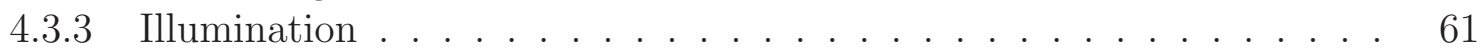

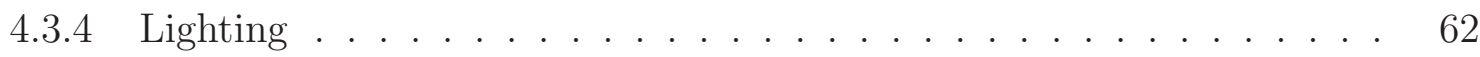

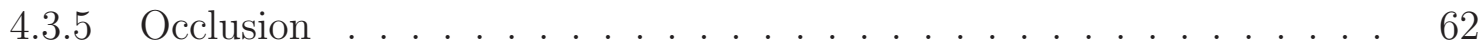

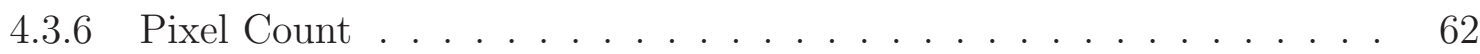

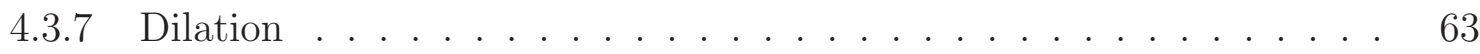

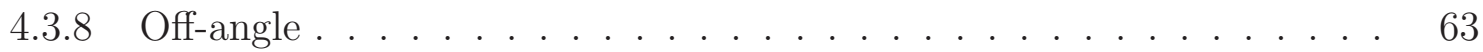

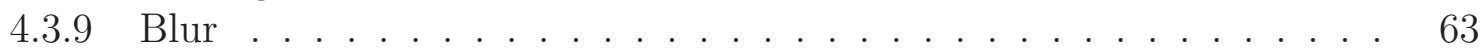

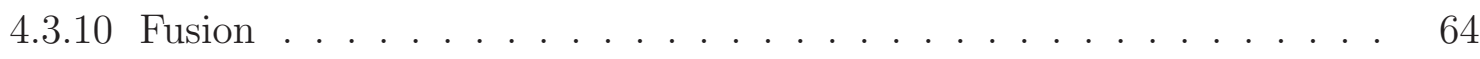

4.4 Results . . . . . . . . . . . . . . . . . . . . 65

4.5 Conclusions . . . . . . . . . . . . . . . . . 67

5 Iris Segmentation $\quad 68$

5.1 Introduction . . . . . . . . . . . . . . . . . . . 69

5.2 Effects of Degradations on Iris Segmentation . . . . . . . . . . . . . . . 71

5.3 Segmentation Methodology . . . . . . . . . . . . . . . . 74

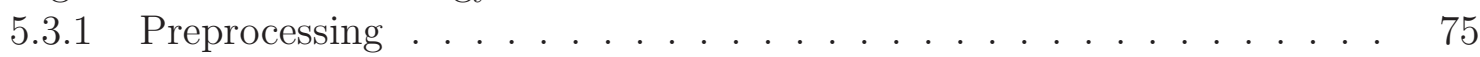

5.3.2 Pupil Segmentation . . . . . . . . . . . . . . . . 82

5.3 .3 Iris Segmentation . . . . . . . . . . . . . . . . . . . . . . . . . . . . . 91

5.3 .4 Occlusion Estimation . . . . . . . . . . . . . . . . . . . 96

5.3.5 Unwrapping . . . . . . . . . . . . . . . . . . . . . . . . . . . 98

5.4 Performance Evaluation . . . . . . . . . . . . . . . . . . 100

5.4.1 Databases and Parameters . . . . . . . . . . . . . . . 100

5.4 .2 Visual Evaluation . . . . . . . . . . . . . . . . . 101

5.4.3 Recognition Performance . . . . . . . . . . . . . . . 104

5.4.4 Automatic Algorithm for Evaluating the Precision . . . . . . . . . . . 107 
5.5 Conclusions and Future Work . . . . . . . . . . . . . . . . . . . 111

6 Iris Encoding 113

6.1 Local Ordinal Binary Extension . . . . . . . . . . . . . . . . . . . . . 113

6.1 .1 Introduction . . . . . . . . . . . . . . . . . . . . . 113

6.1.2 Ordinal Binary Coding and Combining Methodology . . . . . . . . . 114

6.1 .3 Simulated Camera Effects . . . . . . . . . . . . . . . . . . . . . 118

6.1.4 Recognition Performance . . . . . . . . . . . . . . . . . . . . . 121

6.2 Median Filter Based Iris Encoding Technique . . . . . . . . . . . . . . 122

6.2 .1 Introduction . . . . . . . . . . . . . . . . . . . . . 123

6.2 .2 Proposed Algorithm . . . . . . . . . . . . . . . . . . . 125

6.3 Cancelable Iris Biometric . . . . . . . . . . . . . . . . . . . . . . 126

6.3 .1 Introduction . . . . . . . . . . . . . . . . . . . 127

6.3 .2 Methodology . . . . . . . . . . . . . . . . . . . . . 128

6.4 Conclusions . . . . . . . . . . . . . . . . . . . . . . . . . . . . . 131

7 Adaptive Biometric Authentication 132

7.1 Introduction . . . . . . . . . . . . . . . . . . . . . . . . . 132

7.2 Methodology . . . . . . . . . . . . . . . . . . . . . . . . 133

7.2 .1 Quality of Sample $(\mathrm{QS}) \quad \ldots \ldots \ldots$

7.2 .2 Confidence in Scores (CS) . . . . . . . . . . . . . . . . . 135

7.2.3 Quality Sample and Template Features (QST) . . . . . . . . . 136

7.3 Experimental Results . . . . . . . . . . . . . . . . . . . . . . 137

7.3 .1 Neural Network . . . . . . . . . . . . . . . . . . . . . . . . 137

7.3 .2 QS evaluation . . . . . . . . . . . . . . . . . . . . . . . 138

7.3 .3 CS Evaluation . . . . . . . . . . . . . . . . . . . . . . . . . . . . 139

7.3 .4 QST Evaluation . . . . . . . . . . . . . . . . . . . . 139

7.4 Conclusions . . . . . . . . . . . . . . . . . . . . . . . . . . . 141

8 Future Directions and Preliminary Work 142

8.1 Active Iris Recognition . . . . . . . . . . . . . . . . . . . . . . . . . 142

8.1 .1 Introduction . . . . . . . . . . . . . . . . . . . . . . . . 142

8.1 .2 Scenarios . . . . . . . . . . . . . . . . . . . . . . . . . . 144

8.1 .3 Methodology . . . . . . . . . . . . . . . . . . . . . 145

8.1 .4 Discussions and Future Work . . . . . . . . . . . . . . . . . . 152

8.2 Social Connections for Improved Performance of Biometric Systems . . . . . 154

8.2 .1 Introduction . . . . . . . . . . . . . . . . . . . . . 154

8.2 .2 Methodology . . . . . . . . . . . . . . . . . . . 157

8.2 .3 Experiments . . . . . . . . . . . . . . . . . . . . . . . . . . . . . . 159

8.2 .4 Discussions . . . . . . . . . . . . . . . . . . . . . 167

9 Conclusion 168

9.1 Contributions . . . . . . . . . . . . . . . . . . . . . 168

$\begin{array}{ll}\text { References } & 170\end{array}$ 


\section{List of Figures}

1.1 Shown is an iris image captured using a Near-Infrared camera [1]. . . . . . . 2

1.2 Shown is the block diagram of a common iris recognition procedure [1]. . . . 3

1.3 Shown is an iris image captured using a visible light camera [1]. . . . . . . . 5

1.4 Shown are iris images captured at (a) 6, (b) 8 and (c) 10 feet. . . . . . . . 5

1.5 Shown is the organization of this dissertation and the relationship among its chapters. . . . . . . . . . . . . . . . . . 10

2.1 Shown is the cylindrical coordinate system. . . . . . . . . . . . 16

2.2 Shown is the step 1 of iris image synthesis. . . . . . . . . . . . . . . 17

2.3 Shown are the step $2-4$ of iris image synthesis. . . . . . . . . . . . . . . . 18

2.4 Iris images generated by varying "FIBER" parameters (crypts are associated with fibers). . . . . . . . . . . . . . . . . . . 20

2.5 Iris images generated by varying "COLLARETTE" parameters. . . . . . . . 20

2.6 Iris images generated by varying "BASIC" parameters. . . . . . . . . . . . . 21

2.7 Shown are three histogram plots of HDs obtained by varying parameters in one of three major parameter groups. The rightmost histogram (marked in the dark color) was obtained when images generated by varying "FIBER" parameters were compared. The histogram in the middle (marked in the gray color) was obtained by comparing images with random "COLLARETTE" parameters. The leftmost histogram (marked in the light color) was generated by involving images generated by varying a set of "BASIC" parameters. . . .

2.8 A gallery of synthetic iris images generated using model based, anatomy based approach. Iris 4 is a real iris image borrowed from the CASIA data set. . . .

2.9 Shown are three unwrapped and enhanced iris images. The images are samples from (a) CASIA data set, (b) WVU non-ideal iris data set, and (c) data set of synthetic irises generated using our model based approach. . . . . . . . . .

2.10 Original images (left column) and their real components of log-Gabor filtered results (right column). . . . . . . . . . . . . . . . .

2.11 The empirical histogram distributions (dashed lines) and their Bessel $\mathrm{K}$ form approximations (solid lines). . . . . . . . . . . . . . .

2.12 Original images. $I_{1}$ and $I_{2}$ are synthetic iris images, $I_{3}$ and $I_{4}$ are natural images and $I_{5}$ and $I_{6}$ are real iris images. . . . . . . . . . . . . . . .

2.13 The left and the right panels show the dendrogram clustering plot using the distance matrices $d_{I}$ and $d_{K L}$, respectively. . . . . . . . . . . . . . . 
2.14 The clustering results for all 153 images and 4 different filter or filter banks.

2.15 The relationships between different groups of images based on the texture analysis. . . . . . . . . . . . . . . . . . .

2.16 The original image and the other results when different quality factors are added (1: the original image; 2: shot noise; 3: rotation; 4:out-of-focus blur; 5 :motion blur; 6:low contrast). . . . . . . . . . . . . . . .

2.17 The left panel shows the histograms of genuine and imposter HDs characterizing the performance of synthetic iris images processed using the recognition system implemented by Libor Masek. The right panel shows the corresponding ROC curve. . . . . . . . . . . . . . . . . . . . . .

2.18 Shown are the empirical distribution of imposter HDs and the Binomial curve $(N=376)$ that provides the best fit. The HD scores were obtained without performing compensation for rotation.

2.19 The left panel shows the log scale Binomial Minimum Value density function that provided the best fit to the distribution of imposter HDs generated from synthetic data. The right panel compares the Binomial Minimum Value density function and the histogram distribution of HDs obtained from synthetic data. The temples used to perform experiments are of size 20-by-240. . . . .

2.20 The left panel shows the log scale Gumbel extreme value distribution function that provided the best fit to the distribution of imposter HDs generated from synthetic data. The right panel compares Gumbel extreme value distribution function and the histogram distribution of HDs obtained from synthetic data. The temples used to perform experiments are of size 20-by-240.

3.1 An illustration of false color image. . . . . . . . . . . . . . . . . . . . . 44

3.2 Pixel-based prediction. . . . . . . . . . . . . . . . . . . . . . . . . . . . . . . . . . . . . . . . 45

3.3 Neighborhood-based prediction. . . . . . . . . . . . . . . . 46

3.4 Block-diagram of the training procedure. . . . . . . . . . . . . . . . . . . . . . . . . . . . . . . 46

3.5 The block-diagram of the testing procedure. . . . . . . . . . . . . . 47

3.6 (a) The original NIR channel; (b) the R channel; (c) the G/B channel; and (d) the predicted NIR channel using (b) and (c) as the input to the predictive mapping function. . . . . . . . . . . . . . . .

3.7 Unwrapped iris templates: (a) R channel; (b) NIR channel; and (c) the predicted NIR channel. . . . . . . . . . . . . . . . . . . . . . . .

3.8 Unwrapped iris templates: (a) R channel; (b) NIR channel; and (c) the predicted NIR channel. . . . . . . . . . . . . . . . . . . . . . . .

3.9 Performance improvement due to the proposed predictive model. . . . . . . . .

3.10 Performance gains on genuine scores: (a) (Predicted NIR, NIR) vs. (R, NIR); (b) (NIR, NIR) vs. (Predicted NIR, NIR); and (c) (NIR, NIR) vs. (R, NIR).

3.11 The performance improvement due to the proposed predictive model when different segmentations for $\mathrm{R}$ and predicted NIR channels were used. . . . .

4.1 Iris detection for a single frame from the NIR face video: (a) the original frame (from MBGC dataset), (b) clear edges (c) possible iris areas, (d) final candidates, (e) the iris detection result, and (f) the cropped area. . . . . . 
4.2 Shown is the quality map for the video sequence displayed in part in Fig. 4.1. 60

4.3 Example of blur estimation (a) the cropped area, (b) the power spectrum and (c) the results of thresholding. . . . . . . . . . . . . . . .

4.4 ROC curves for ICE2005 dataset (a) selecting images using pupil segmentation score; (b) selecting images using dilation measure; and (c) selecting images using minus blur measure. . . . . . . . . . . . . . . . . . . . . .

4.5 ROC curves for ICE2005 dataset (a) selecting matching scores using interlacing measure; (b) selecting matching scores using illumination measure; (c) selecting matching scores using pixel count measure; and (d) selecting images using off-angle measure. . . . . . . . . . . . . . . . . . . . . . .

5.1 Segmentation of occluded iris images: (a) the original image $I_{1}$ (from WVU dataset), (b) correct segmentation for $I_{1}$, (c) the original image $I_{2}$ (from WVU dataset), (d) incorrect segmentation of $I_{2}$. The occlusions are due to eyelids.

5.2 Segmentation of occluded iris images: (a) the original image $I_{1}$ (from CASIA I dataset), (b) correct segmentation of $I_{1}$, (c) the original image $I_{2}$ (from CASIA I dataset), (d) incorrectly segmented $I_{2}$. The occlusions are due to eyelashes.

5.3 Segmentation of iris images containing strong specular reflections: (a) the original image $I_{1}$ (from CASIA III dataset), (b) correct segmentation of $I_{1}$, (c) the original image $I_{2}$ (from CASIA III dataset), (d) poor segmentation of $I_{2}$ due to the presence of strong specular reflections.

5.4 Segmentation of images with low contrast: (a) the original image $I_{1}$ (from CASIA II dataset), (b) correctly segmented $I_{1}$, (c) the original image $I_{2}$ (from CASIA II dataset), (d) fair segmentation of $I_{2}$. . . . . . . . . . . . . . . . .

5.5 Segmentation of unevenly illuminated images and images with low contrast: (a) the original image $I_{1}$ (from WVU dataset), (b) correct segmentation of $I_{1}$, (c) the original image $I_{2}$ (from WVU dataset), (d) incorrect segmentation of

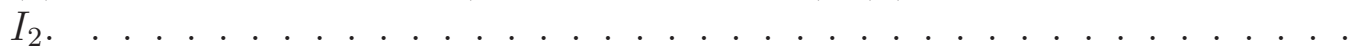

5.6 Segmentation of out-of-focused images: (a) the original image $I_{1}$ (from ICE dataset), (b) segmentation results for $I_{1}$, (c) the original image $I_{2}$ (from ICE dataset), (d) poor segmentation of $I_{2} \ldots \ldots \ldots \ldots$

5.7 Segmentation of motion blurred iris images: (a) the original image $I_{1}$ (from WVU dataset), (b) segmentation result for $I_{1}$, (c) the original image $I_{2}$ (from WVU dataset), (d) fair segmentation result for $I_{2} \ldots \ldots$. . . . . . . . . .

5.8 Segmentation of the off-angle iris: (a) the original frontal view image $I_{1}$ (from WVU Off-angle dataset), (b) segmentation result for $I_{1}$, , (c) the original 30 degree off-angle image $I_{2}$ (from WVU Off-angle dataset), (d) fair segmentation

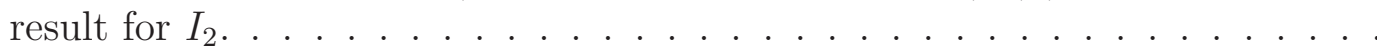


5.10 Inpainting steps: (a) the original image, (b) mask $k_{1}$ of the potential pupil area, (c) gaps in mask $_{p 1}$ are filled to obtain the mask mask $k_{p 2}$, (d) rough specular reflection mask $_{s}$ is obtained using the threshold $\gamma$, (e) expanded specular reflection mask $_{s}$, (f) combination of (c) and (e), (g) the intersection mask $_{p 2} \bigcap$ mask $_{s}$ of two masks, (h) result of initial inpainting, (i) refined inpainting result after intensity adjustment, (j) rough specular reflection mask $_{s 1}$ obtained using the threshold $\gamma$ applied to (i), (k) refined and expanded specular reflection mask $_{s 1}$, (l) final result of inpainting, (m) final specular reflection mask used to generate the template mask, (n) template mask. . . . . . . . .

5.11 In this example, six pupil candidates are selected based on a circular shape of bright regions. Then the intensity information is used to select the optimal candidate with the largest intensity value. . . . . . . . . . . . . .

5.12 "Spike" removal: (a) scanning the boundary, (b) "valley" type "spike", (c) "longhorn" type "spike". Dotted curves in (b) and (c) are the boundaries of the circles that are used to remove "spikes." . . . . . . . . . . . . . . .

5.13 "Spike" removal: (a) the original image, (b) the estimated pupil boundary, (c) removing the "spike", (d) the refined boundary after the "spike" removal.

5.14 Breaking unwanted connections: (a) the original image, (b) the connected mask, (c) the erosion result, (d) select the pupil region from the eroded result using the location information of the optimal candidate, (e) the dilated pupil region, (f) the result of intersecting (a) and (e). . . . . . . . . . .

5.15 Concavity removal: (a) the original image, (b) the initial concave pupil mask, (c) the convex hull result, (d) the result of ellipse fitting procedure using only unaffected pupil boundary.

5.16 Curve fitting: (a) the circle model, (b) the unrotated ellipse model, (c) the control point based model, (d) the rotated and translated ellipse model. . . .

5.17 Ellipse fitting: (a) the original image, (b) refined pupil mask, (c) specular reflection mask, (d) ellipse fitting result taking into account specular reflections, (e) the error (the XOR between ellipse fitting with the original refined pupil mask), (f) the edges detected near the refined pupil boundary, (g) selected partial boundary using 4 conditions listed above, (h) the new ellipse fitting result using selected partial boundary, (i) the final segmentation result. . . .

5.18 Image translation procedure: (a) the original image, (b) new image after translation and impainting, (c) enhanced image, (d) segmented iris, (e) unwrapped template, (f) unwrapped template mask. . . . . . . . . . . . .

5.19 A rotated ellipse (left) and its representation in a circular coordinate system.

5.20 (a) The original image showing an off-angle iris and (b) the same iris transformed into the circular coordinate system and unwrapped. The regions E1, E3, and E5 contain the true boundary between the iris and sclera and are used to find the parameters of the ellipse to be fitted into the iris. . . . . . .

5.21 The relationship between the off center distance in pixels and the off angle in degrees. . . . . . . . . . . . . . . . . . .

5.22 The result of segmentation (a) before contrast balancing and (b) after application of contrast balancing. . . . . . . . . . . . . . . . . 
5.23 Contrast balancing procedure: (a) the original image with results of pupil segmentation imposed onto the image, (b) the unwrapped image, (c) the iris boundaries are detected separately for left and right portions of the iris, (d) the result of the final iris detection with different contrast compensation weights added on the both sides. . . . . . . . . . . . . . . . .

5.24 The evolution of the occlusion mask: (a) the original image, (b) the result of the initial edge detection, (c) the edge information after smearing, (d) the result of combination of the mask and the outcome of iris segmentation, (e) the area selected based on connectivity information, and (f) the final result. .

5.25 The evolution of the occlusion mask: (a) the original image, (b) the result of the initial edge detection, (c) occlusion mask obtained without illumination preprocessing, (d) image after illumination compensation, (e) the result of horizontal edge detector, (f) occlusion mask obtained after application of illumination compensation. . . . . . . . . . . . . . . . .

5.26 Criteria used for visual evaluation of the proposed segmentation algorithm. .

5.27 Examples of: (a) good, (b) fair, (c) poor, and (d) bad segmentations. . . . .

5.28 Examples of correctly segmented iris images from WVU and WVU-OA datasets. 104

5.29 Segmentation of occluded iris images: (a) the original image (from WVU dataset), (b) circular fit due to a simple edge-based approach (dashed line) and elliptical fit due to our algorithm (solid line). . . . . . . . . . . . . .

5.30 ROC performance of (a) right and (b) left eye for ICE data. IrisBEE is a version of Masek's algorithm. IrisBEE composed of segmentation, encoding, and matching algorithms was used as a baseline algorithm in ICE 2005. The solid $\mathrm{ROC}$ curves on the left and right panels in the figure are obtained using the segmentation algorithm proposed in this chapter and encoding and matching algorithms of the IrisBEE. . . . . . . . . . . . . . . . . . . . 106

5.31 The distribution of the off-center distances for ICE dataset. . . . . . . . . . . 107

5.32 An example of large off-center distance image from ICE dataset (243265.tiff). 107

5.33 The steps performed by the automatic algorithm for evaluation of precision of iris segmentation: (a) the original image, (b) the unwrapped template, (c) the unwrapped mask, (d) the extended portion of unwrapped iris on the pupil side, (e) the result of edge detection on the pupil side, (f) the extended portion of unwrapped iris on the sclera side, (g) the result of edge detection on the sclera side. . . . . . . . . . . . . . . . . . . . .

6.1 LBP vs. LFOBC: (a) the intensity values of pixel $p_{0}$ and its neighbors, (b) LBP code: 11010000, (c) LFOBC code: 110100001000000000000110100000000110 , or $001011110111111111111001011111111001 \ldots \ldots \ldots \ldots$. . . . . . . .

6.2 LBP vs. LFOBC: (a) the pixel $p_{0}$ and its neighbors, (b) the calculation of LBP, (c) the calculation of LFOBC. . . . . . . . . . . . . . . . 117

6.3 Testing templates: (a) random pattern, (b) natural image, (c) iris template. 119

6.4 Performance comparison of different coding schemes for CASIA-3 dataset. . . 123 
6.5 The block diagram of median filtering based encoding: (a) the original iris pattern; (b) 2D median filter results; and (c) the binary code generated from the filtered results. . . . . . . . . . . . . . . . . . . . . . .

6.6 The block diagram of GRAY-COMBO: (a) the original iris pattern; (b) random shifting; (c) random split (in this case, just split to the top half and the bottom half); and (d) combine together (using plus here). . . . . . . . . .

6.7 The block diagram of BIN-COMBO: (a) the original iris pattern; (b) random shifting; (c) random split (in this case, just split to the top half and the bottom half); and (d) combine together (using XOR here).

6.8 The block diagram of GRAY-SALT: the original iris pattern and the random noise pattern are combined together to generate the new cancelable iris pattern.130

6.9 The block diagram of BIN-SALT: the original iris code and the random binary noise pattern are combined together to generate the new cancelable iris code.

7.1 Illustration of the Confidence in Scores (CS) . . . . . . . . . . . . . . . . 136

7.2 Combining quality vectors and templates. . . . . . . . . . . . . . 137

7.3 Performance improvement achieved by selecting only images with high QS values. . . . . . . . . . . . . . . . . . . . . .

7.4 Performance improvement by selecting only images with a quality value larger than a certain quantile. . . . . . . . . . . . . . . . .

7.5 Performance improvement achieved by selecting only matching scores with high CS values. . . . . . . . . . . . . . . . . . . .

7.6 Performance improvement by selecting only matching scores with a confidence level higher than a certain quantile. . . . . . . . . . . . . . . . . . . . 140

8.1 The setup at an airport. . . . . . . . . . . . . . . . . . . 145

8.2 A potential setup at a security check point. . . . . . . . . . . . . . . 145

8.3 The technology used in the Kinect ${ }^{\circledR}$. . . . . . . . . . . . . . . . . . . . . 146

8.4 The background (a) and its corresponding depth map (b). . . . . . . . . . . 147

8.5 Noise removal: (a) the color scene image, (b) the original depth map, (c) differences from the trained background, (d) the original foreground mask, (e) eroded mask where only parts of true subject are left and (f) the final foreground mask. . . . . . . . . . . . . . . . . . . .

8.6 Head detection: (a) the initial score based on the height, (b) the center of mass, (c) the penalty for the off-center, (d) the combination of (a) and (c), (e) the penalty for the edges and (f) the final head(forehead) candidate. . . .

8.7 Head detection when there are connections: (a) the color scene image, (b) the original depth map, (c) the narrow connection between two subjects and (d) separating subjects using vertical cuts. . . . . . . . . . . . . .

8.8 Head detection when there are overlaps: (a) the color scene image, (b) the original depth map, (c) all clear distance changes in the scene, (d) the expanded selection, (e) the "skeleton" and (f) the final successful head detection result.

8.9 The face detection result. . . . . . . . . . . . . . . . . . . . 151

8.10 The diagram of the demo. . . . . . . . . . . . . . . . . . 152

8.11 The robotic arm is following the position of the head in the live demo. . . . 153 
8.12 The visualization of a small social network. . . . . . . . . . . . . . . . 155

8.13 The social network match will support the results of biometric identification. 156

8.14 The flow chart of a biometric verification system relying on a social network. 158

8.15 The credit card application procedure. . . . . . . . . . . . . . . . 159

8.16 The flow chart of a social network assisted biometric verification. . . . . . . 160

8.17 The distribution of genuine and imposter scores obtained using 20,000 classes. 161

8.18 The Receiver Operating Characteristic(ROC) curve based on 20,000 classes. 161

8.19 The 3 possible outcomes in the identification scenario. . . . . . . . . . . . . 162

8.20 The trend of decreasing matching scores when the number of referrals increases. 164

8.21 The trend of increasing number of referrals when the verification threshold is decreasing. . . . . . . . . . . . . . . . . . 165

8.22 The hierarchical structure in the company. . . . . . . . . . . . . . . 165

8.23 The plot of the hierarchy network. . . . . . . . . . . . . . . 166

8.24 The number of referrals needed for each grade level to access class 5 files. . . 167 


\section{List of Tables}

$2.1 \quad$ Public iris databases . . . . . . . . . . . . . . . . . . . . . . . . 12

2.2 The Bessel K parameters estimated from images filtered by log-Gabor filter . 27

2.3 Degree of freedom . . . . . . . . . . . . . . . . . . . . 34

2.4 The threshold values and its effect on FAR. The results are obtained using Binomial Minimum Value distribution (with 208008 imposter comparisons) 38

2.5 HD threshold and its effect on FARs. The results are obtained using Gumbel extreme value distribution (with 208008 imposter comparisons) . . . . . . . 40

3.1 The results of three cross comparisons _ . . . . . . . . . . . 50

3.2 The results of three cross comparisons with imperfect relative segmentation of R and Predicted NIR channels . . . . . . . . . . . . . . . . . . 52

5.1 Parameter settings for different datasets . . . . . . . . . . . . . . . . 101

5.2 Success rate in segmentation . . . . . . . . . . . . . . . . . . . . . . 104

5.3 The results of manual performance evaluation . . . . . . . . . . . . . . 104

5.4 Complexity (resolution vs. running time (in seconds)) . . . . . . . . . . 106

5.5 Summary of decisions made by automatic algorithm . . . . . . . . . . . . . 110

5.6 Summary of decisions made by manual evaluation . . . . . . . . . . . . 110

5.7 Automatic vs. manual . . . . . . . . . . . . . . . . . . . . . . . 111

6.1 All possible Hamming distance outcomes for a pair of pixels $p_{0}$ and $p_{0}^{\prime}$ using log-Gabor binary encoding scheme . . . . . . . . . . . .

6.2 The new normalized Hamming distance calculation scheme for a pair of pixels $p_{0}$ and $p_{0}^{\prime}$ seamlessly integrate the ordinal binary codes into the log-Gabor binary code . . . . . . . . . . . . . . . . .

6.3 Recognition capabilities of five encoding techniques in the presence of random noise (case of uniform distributed noise and random textures . . . . . . . . 120

6.4 Recognition capabilities of five encoding techniques in the presence of random noise (case of uniform distributed noise and Brodatz textures) . . . . . . . . 121

6.5 Recognition capabilities of five encoding techniques in the presence of random noise (case of uniform distributed noise and iris textures) . . . . . . . . . 122

6.6 Performance comparison for out-of-focus blur . . . . . . . . . . . . . . . 122

6.7 Performance comparison for motion blur . . . . . . . . . . . . . . 123

6.8 Performance comparison of five encoding schemes using d-prime $\left(d^{\prime}\right)$ and EER 124 
7.1 Performance with/without quality factors . . . . . . . . . . . . . . 141

8.1 Number of classes for each case . . . . . . . . . . . . . . . . . . . 162

8.2 The average frequencies for different scenarios (threshold: 0.32 ) . . . . . 163

8.3 Thresholds for different classes . . . . . . . . . . . . . . . . . . . . . . . 164

8.4 Number of people at each level . . . . . . . . . . . . . . . . . . . . . . . . . . . . . . . . . . . . . . . . . . . .

8.5 The average number of referrals needed for each scenario . . . . . . . . . 167 


\section{Chapter 1}

\section{Introduction}

In ancient Greek, "bios" means life and "metron" means measure. Two words combine to form a portmanteau word, "biometrics", which means measuring some body's physical or behavioral characteristics, such as face, gait or voice, to recognize a person. In general sense, "biometrics" is related to "biometry" which is statistical method of processing any biological data. It is in the past 30-40 years that the meaning of biometrics narrowed down to few physiological or behavioral traits of subjects. These traits (or to be more precise, measurements of the traits) are processed, encoded and matched against similar data in a database. The system implementing this steps is called biometric system. Biometric systems can identify or verify individuals depending on requirements to the system. Verification systems confirm or reject identity of a single individual, while identification systems select the best matching subject (based on his biometric measurements) among a long list of subjects.

This dissertation is devoted to a single biometric known as iris. Iris biometric systems use the "colored ring of tissue around the pupil" to identify a subject [2]. Fig. 1.1 shows an iris image acquired using a NIR (Near-Infrared) illumination source and camera. The pupil region generally appears darker than the iris area unless "red-eye" effect is observed. This happens when the light source is too close to the sensor-pupil axis. The pupil area in this case appears as a white circle. Under NIR illumination iris shows a rich pattern of ridges, which we perceive as coming from projections of fibers and muscles. The central pupillary zone normally contains a more complex texture pattern than the outer ciliary zone. The border 


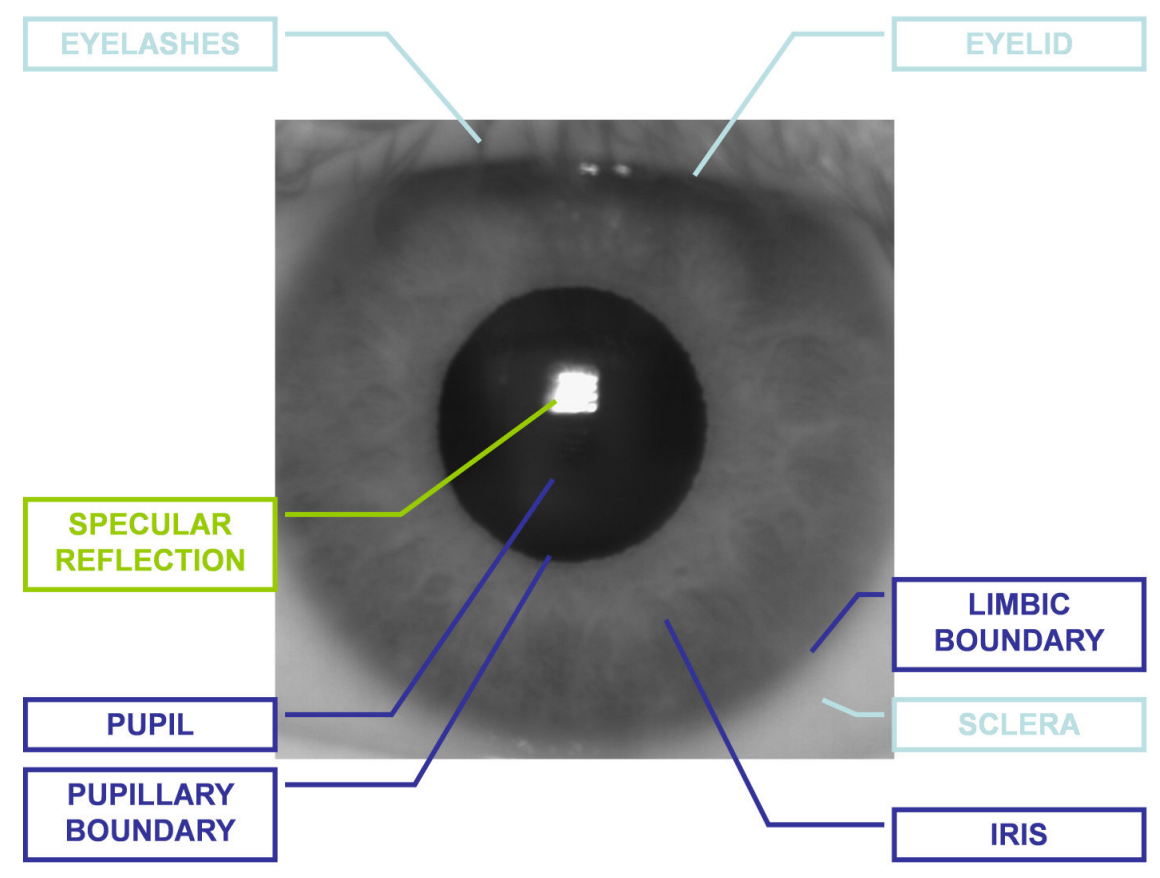

Figure 1.1: Shown is an iris image captured using a Near-Infrared camera [1].

between these two regions is called collarette. The iris texture is developed at embryonic stage of our development, and due to genetic and environmental influence it is believed to be unique for each individual and each eye [3]. The appearance of the iris is relatively constant during lifespan for most of individuals.

The idea of using iris pattern for recognition of individuals can be attributed to a prominent eye surgeon from St. Paul, Frank Burch, who in 1936 promoted the idea that the complex iris patterns could serve as a kind of optical fingerprint to recognize an individual. In 1987, two other ophthalmologists, Aran Safir and Leonard Flom were awarded a patent for using the iris as a means for human identification $[4,5]$. However only after the success of John Daugman's automated iris recognition algorithm, which was awarded a patent in 1995, iris recognition gains acceptance among researchers.

Compared to other biometrics, iris has a number of advantages: (1) it is an internal organ that is well protected against damage, and no contact is necessary when iris is scanned for a usable sample; (2) good quality iris images can be easily segmented; (3) the unique feature set extracted from irises is suitable for large-scale recognition.

Similar to other biometric systems, iris recognition systems can operate in two different 


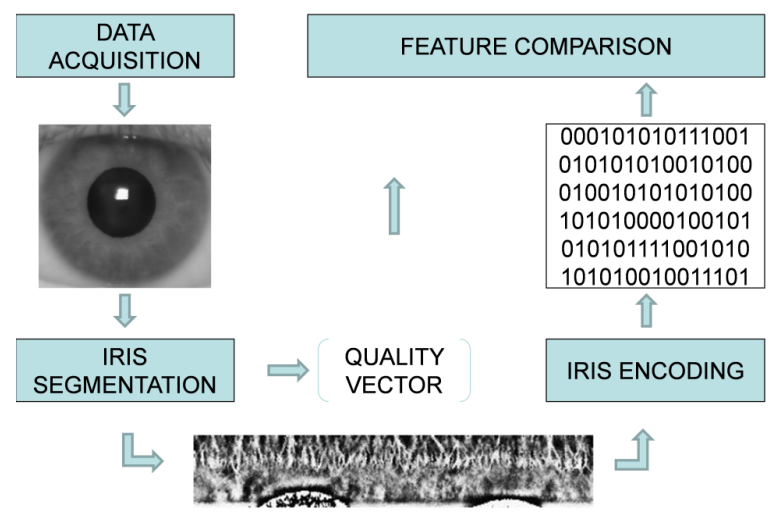

Figure 1.2: Shown is the block diagram of a common iris recognition procedure [1].

modes: "verification" and "identification." In a verification scenario, a person claims a particular identity, and the system uses his/her iris pattern to verify or reject the claim. Verification is performed by matching an iris sample acquired at the time of the claim against the iris sample stored in a database during enrollment procedure. If the two samples match well enough, the identity claim is verified, and if the two samples do not match well enough, the claim will be rejected.

In an identification scenario, an iris sample is presented without any associated identity claim. For example, a sample may be captured by a hidden camera. The task is to identify the unknown iris sample by comparing it to each entry in a database of enrolled irises. The set of previously enrolled samples with identity tags attached is often called a gallery, and the unknown iris sample is often called a probe. The closest match is used to identify the unknown identity.

Both modes of operation involve the same iris processing blocks shown in Fig. 1.2: (1) iris is captured using a NIR iris scanner, (2) quality of iris image or video is evaluated using a quality block, (3) iris area is then segmented from the background, (4) the segmentation result and its corresponding quality and segmentation scores are passed to the encoder for the generation of an informative and compact representation (called template), (5) finally, the extracted representation is fed to the matcher for the evaluation of the matching score. 


\subsection{Iris Acquisition}

Iris acquisition set up determines quality of the iris data. Different choices of the camera sensor, lens, illumination and capture distance will result in data of varying quality.

\subsubsection{Successful Commercial Systems}

Almost all commercial iris recognition systems are using NIR illumination sources and NIR cameras. This is because color cameras are not as effective as NIR cameras in terms of capturing the textural information in iris. Visually dark brown (almost black) irises do not display much color variation and thus contain almost no information useful for iris recognition.

Due to the requirement to be low cost iris recognition systems often involve inexpensive and thus low quality sensors and optics. This considerably limits capture distances as well as quality of captured data. A strong source of NIR illumination is another important component. However, pupil does not respond on the NIR illumination the same way as it responds on visible light. Therefore, safety issue becomes more restrictive in a system operating in NIR range. To protect the eye from overheating and thus from damage, the strength of the illumination is required not to exceed a predetermined threshold [6].

\subsubsection{Visible Light}

As was mentioned before, dark irises contain almost no useful information for iris recognition. However, a large number of surveillance systems rely on imaging in visible range. How to use the color and texture information in color iris images is an important and relatively new research topic [7].

\subsubsection{Long Distance}

Surveillance applications imply that iris information is captured from a distance longer than 2-3 feet, which is a typical distance for personal and security applications. Under this scenario, the quality of the iris image will be heavily degenerated because of the limitation of 


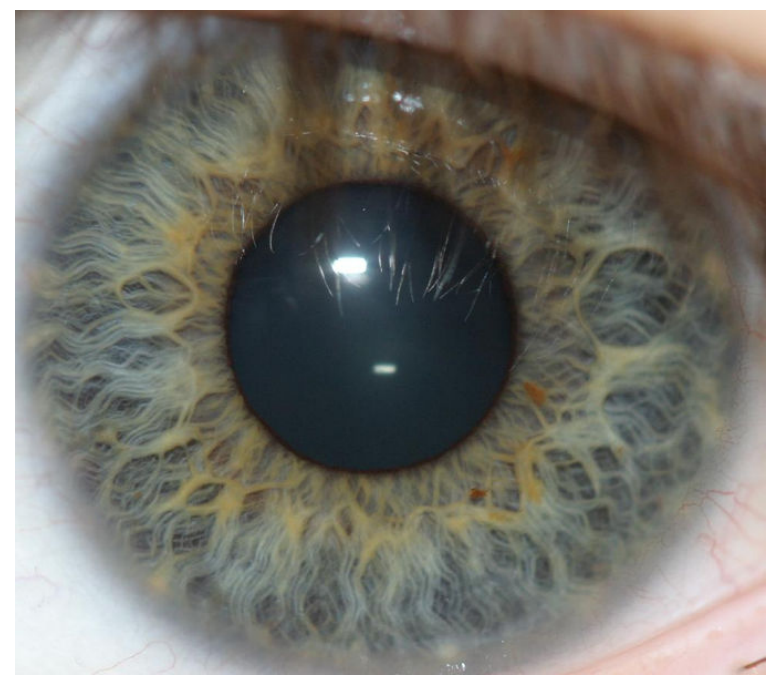

Figure 1.3: Shown is an iris image captured using a visible light camera [1].

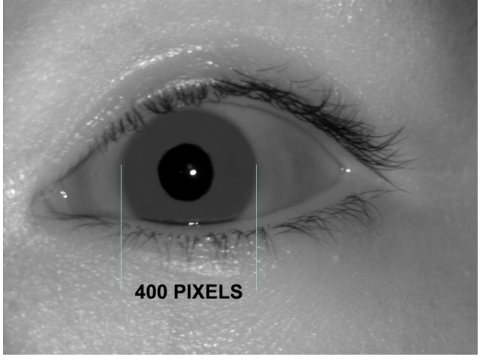

$(a)$

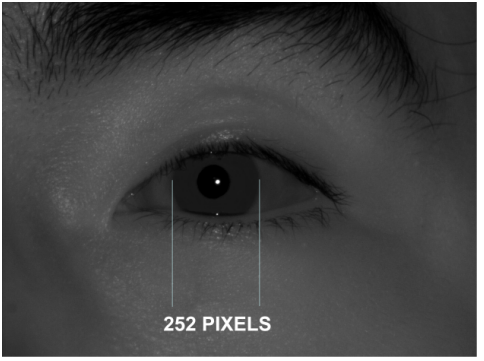

(b)

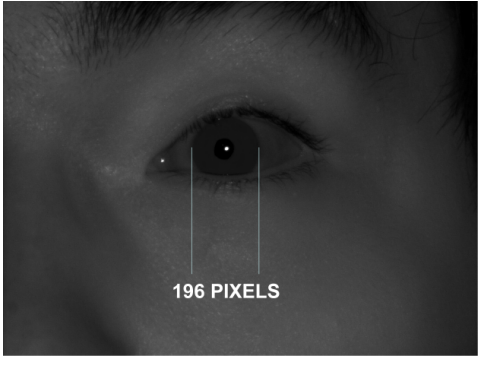

(c)

Figure 1.4: Shown are iris images captured at (a) 6, (b) 8 and (c) 10 feet.

the capture device (its aperture, depth of field, etc.) and thus, getting acceptable recognition performance will be very challenging.

\subsubsection{Generated Data}

Reliable testing of newly designed iris recognition algorithms requires a large amount of relevant data. These data have to be diverse both in terms of subject's presentation (distance,

angle of view, etc.) and in terms of environmental conditions. Since no a large dataset of iris images exists, which encompasses all these requirements, generation of synthetic data for testing could be a potential solution. A synthetic database is easy to access, avoids the privacy concerns and can be easily scaled up. However, because of the limitation of the 
algorithm, synthetic data can not exactly mimic the real situations. Therefore, synthetic data have to be used with caution.

\section{$1.2 \quad$ Iris Quality}

The performance of the system is always determined by the quality of the input data. We propose a number of potential approaches on how to evaluate the quality, how to select good quality data, and how to use the quality scores.

\subsubsection{Selecting the Best Quality Iris Image}

When the input of the system is a video sequence, frames with the best quality need to be selected. While the precise segmentation is time consuming and some quality factors are only available after the segmentation, only out-of-focus is used as the selecting criterion in the current implementation.

\subsubsection{Processing Iris Data}

Whenever the input image is selected, it can be enhanced and fully processed. At this stage, segmentation based quality factors, such as occlusion and dilation, can be evaluated. These quality scores will be passed to the matching block of iris recognition system to improve its recognition performance.

\subsubsection{Adaptive Biometric Authentication}

Some quality measurements can be also used as weak features in biometrics. Given enough training data and well designed quality factors, not only the recognition performance of the feature set but also the confidence of the matching score can be predicted. Therefore, predicted quality of data as well as quality measures can be used to enhance performance of biometric systems. 


\subsection{Iris Segmentation}

Iris segmentation is perhaps the most important processing step, since the precision of segmentation effects recognition performance. The region of interest needs to be precisely segmented from the background. In some cases it may not lead to a success. When the capture condition is assumed non-ideal or it is unknown, such as a surveillance scenario, the full set of enhancement technologies has to be involved to segment the iris. A series of image processing procedures will be used to compensate all possible low quality factors. At last a robust segmentation performance evaluation procedure is added to let the user know how good the segmentation result is.

\subsection{Encoding}

Encoding is performed to extract salient features representing an iris. This step ensures that data are compactly represented and also ensures that noise introduced by acquisition system is suppressed or removed.

Many different filters have been suggested to extract features: Gabor filters [8], modified Log-Gabor filters [9], Gaussian filter [10], Laplacian-of-Gaussian filter [11] [12], dyadic wavelet transform [13], discrete cosine transform [14] and wavelet packets [15] [16] [17]. Extracted features were quantized to two levels yielding binary codes. Techniques other than filter-based approaches can also be used to generate binary iris codes, for example, local histogram equalization and a quotient thresholding [18], [19], [20].

Instead of binary presentations, real-valued feature vectors can be generated using independent component analysis (ICA), principal component analysis (PCA), linear discriminant analysis (LDA), direct linear discriminant analysis (DLDA), local binary patterns (LBP) and even histograms [21] [22] [23] [24] [25] [26] [27].

From log-Gabor filter based binary iris code, we developed an encoding scheme, which incorporates ordinal information into the expended binary code. This expansion is especially useful for low quality area where only one out of two bits matches. Another encoding approach is based on median filters. Combinations of a series of median filters enhances iris 
texture and removes illumination variations at the same time.

The idea of "cancelable biometrics" originally proposed by Nalini Ratha and his colleagues was also applied to iris biometric. This is an approach to solve the privacy and security problems simultaneously.

\subsection{Performance}

Since biometric systems are a case of more general pattern recognition systems, all methods to evaluate performance of pattern recognition systems directly apply to analyze performance of biometric systems. The most common performance measures are summarized below.

Similarity scores (distance measures) are used to express the similarity (difference) between iris patterns. If Hamming distance is used, then the lower the score is, the higher is the similarity between two binary templates. Access to the system is granted only when the distance is lower than a certain threshold. Under the optimal conditions, genuine distances (scores of patterns from the same subject known by the system) should always be lower than the distances of impostors. If this would be true, a single threshold, that separates the two groups of distance metrics, could be used to differ between genuine users and impostors. Due to clutter, noise and errors, this assumption may fail for real world biometric systems. In some cases impostor patterns generate the values of the distance metric that are lower than the values of the distance metric generated by genuine patterns. In this case, because the threshold is already chosen as a system parameter, some classification errors may occur.

The "decidability" index is a single number performance measure, which tells how well the two distributions are separated [8]. If the means of the genuine distribution and the imposter distribution are $\mu_{1}$ and $\mu_{2}$, and their standard deviations are $\sigma_{1}$ and $\sigma_{2}$, then the decidability index, $d^{\prime}$, is defined as

$$
d^{\prime}=\frac{\left|\mu_{1}-\mu_{2}\right|}{\sqrt{\frac{\sigma_{1}^{2}+\sigma_{2}^{2}}{2}}} .
$$

The fraction of the falsely accepted patterns divided by the number of all impostor patterns is called False Acceptance Rate (FAR). The fraction of the number of rejected 
genuine patterns divided by the total number of genuine patterns is called False Rejection Rate (FRR). For both FAR and FRR, their values lies in between zero and one. Receiver operating characteristic (ROC) curve is defined as a plot of FRR versus FAR parameterized by threshold values. If histograms of genuine and imposter distance metrics overlap, the FAR and FRR curves plotted as functions of the threshold intersect at a certain point. The point of intersection is called Equal Error Rate (EER). The area under the ROC curve is another single point performance measure.

\subsection{Applications}

Iris recognition can be used in almost any biometric applications. However, the system needs to be designed according to the unique property of the iris biometric. How biometric systems can be combined with existing systems, and how a piece of additional information other than biometrics can be used to boost the system performance, are also illustrated in this dissertation.

\subsection{Organization of this Dissertation}

As illustrated in Fig. 1.5, the following chapters of this dissertation will be in the order of data processing: from data acquisition, segmentation, encoding to designing a matcher and applications. Ch. $2-7$ of this dissertation have been externally published. Newly developed and yet unpublished results are described in details in Ch. 8. Ch. 9 concludes the dissertation with a summary of contributions. 


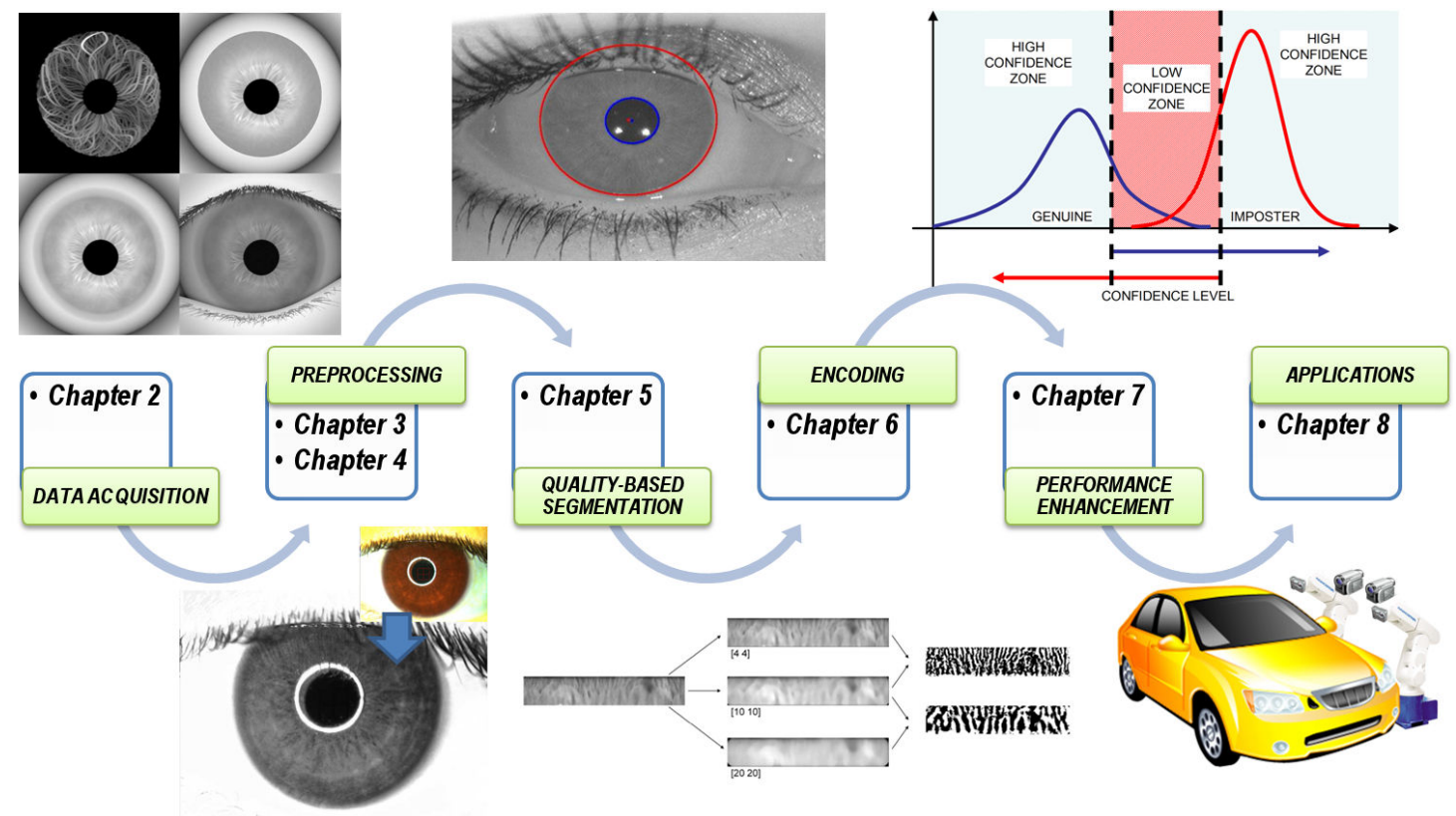

Figure 1.5: Shown is the organization of this dissertation and the relationship among its chapters. 


\section{Chapter 2}

\section{Synthetic Iris}

The coverage strength of iris biometric (also known as scalability) is an important unsolved problem. This is partially due to the lack of a large scale database of iris images, which can be used to evaluate performance of existing iris recognition algorithms. Our approach to solve the problem is summarized in the chapter below.

\section{$2.1 \quad$ Introduction}

Most existing iris biometric systems or algorithms claim to have exclusively high performance. However, since there are no publicly available large scale and even medium size data sets, neither of the algorithms has undergone extensive testing. There are several data sets of frontal view iris images presently available for public use [28], [29], [7], [30], [31]. Table 2.1 provides a brief summary of those databases.

Finally all algorithms should be tested on large real iris data sets to verify their performance. But the issues of security and privacy let both biometrics information providers and biometrics researchers think twice before they provide or publish biometrics information to the public, and then slow down the speed of collecting and publishing biometrics data, such as iris images, from volunteer. While biometrics technologies become much maturer than before, the probability that iris information is used in passport or credit card verification will be very high in the future. Then iris information, especially high resolution pictures, will become the most important personal information and should be safely protected. But it can 
Table 2.1: Public iris databases

\begin{tabular}{|c|c|c|c|c|}
\hline $\begin{array}{c}\text { Database } \\
\text { Name }\end{array}$ & $\begin{array}{c}\text { Database } \\
\text { Size }\end{array}$ & $\begin{array}{c}\text { \# of } \\
\text { Classes }\end{array}$ & $\begin{array}{c}\text { \# of Images } \\
\text { per Class }\end{array}$ & $\begin{array}{c}\text { Color or } \\
\text { Gray Scale }\end{array}$ \\
\hline CASIA-I & 756 & 108 & 7 & gray \\
\hline CASIA-III-device1 & 1200 & 60 & 20 & gray \\
\hline CASIA-III-device2 & 1200 & 60 & 20 & gray \\
\hline CASIA-III-Interval & 2655 & 396 & NA & gray \\
\hline CASIA-III-Lamp & 16213 & 819 & NA & gray \\
\hline CASIA-III-Twins & 3183 & 400 & NA & gray \\
\hline ICE2005 & 2953 & 244 & NA & gray \\
\hline MMU1 & 450 & 90 & 5 & gray \\
\hline MMU2 & 995 & 199 & 5 & gray \\
\hline WVU-off-angle & 560 & 140 & 4 & gray \\
\hline WVU & 2453 & 359 & NA & gray \\
\hline UBIRIS & 1877 & 241 & NA & color \\
\hline UPOL & 384 & 128 & 3 & color \\
\hline BATH-free & 1000 & 50 & 20 & gray \\
\hline
\end{tabular}

not be anticipated that all iris images in public databases will be properly used, even they are not tagged. Some people may use them to break into an iris biometric system by using an iris picture of a person who already enrolled in that biometric system, or just enroll into an iris biometric system as a new user for the purpose of hiding his or her own biometrics information. Compared to credit card or social security number, iris or other biometrics information also has a disadvantage: if you lose its privacy, you can not get a new one.

With the lack of data and the thirst for the performance evaluation, an optional solution to the problem of extensive algorithm testing is possible: synthetically generating a large scale data set of iris images. This chapter proposes a model based, anatomy based method to synthesize iris images. The purpose of this work is not to provide a large database of iris images that will replace the real world data, but rather provide an option to compare efficiency, limitations, and capabilities of newly designed iris recognition algorithms through their testing on this large scale synthetic iris data set.

We are aware of the advice by Mansfield and Wayman [32] to avoid adding synthetic data to the test set, or adding artificial noise to data for scenario testing in order to avoid the resulting bias that often makes the results difficult to interpret. Although controllable 
random parameters can be tuned to simulate a certain collected real iris database, the use of our data set can only be viewed as an option to get a quick estimate of recognition performance for a designed iris recognition system. Some synthetic data sets may provide optimistic or pessimistic bounds on recognition performance because of their bias compared to collected real iris images. Furthermore, the results of testing on synthetic data can always be verified against the results predicted by performance extrapolation methods using a small but real data set (see for example [33]).

The first methodology for generating synthetic iris images has been proposed by Cui et al. [34], where a set of principle components from downsampled real iris images was calculated using Principal Component Analysis (PCA) method. Principle components were further combined with weights to determine the center of each class. Disturbances were added to the center images to generate a number of low resolution iris images from the same iris class. Low resolution results were enhanced using a super-resolution method to get the high resolution iris images. Another method for the generation of synthetic iris images based on application of Markov Random Field has been recently developed at WVU [35] and offered as an alternative to the model based, anatomy based method described in this chapter. Lefohn et al. [36] developed an ocularist's approach using the computer vision technology for the purpose of both the ocular prosthetics and entertainment industries. In their work a set of textured layers was used to render each iris. Wecker et al. [37] combined characteristics of real irises to augment existing real iris databases. In their work a multi-resolution technique known as reverse subdivision was used to capture the necessary characteristics.

When generating synthetic iris images, the problem that one faces is to define a measure of "realism" and make the database suitable for biometrics testing. What is the set of requirements that a synthetic iris has to satisfy in order to be recognized and treated as a physically collected iris image? The conclusion could be: (i) each iris image should look like a real iris; (ii) the data set should have the similar statistical characteristics to those of real irises. Real iris patterns are so anatomically complex that it is nearly impossible to mathematically describe any particular one. Thus, standards of realism will be limited to some degree. However, simplified mathematical models of iris anatomical structures may be used for iris structure generation. 
In this work, an anatomy based model is adopted for the generation of iris images and the performance of synthetic irises is evaluated in three different levels. Extensive anatomical studies of the iris, including the study of ultra-structure images and high-resolution images [38] [39], structure and classification of irises due to iridology [40], and models available for the iris [34] [36] were conducted. As a result of observational study, a few common visual characteristics of irises have been found:

- Most iris images used in biometrics research are infrared images, which emphasize on structural texture more than color information and are more constant over time;

- Radial fibers constitute the basis for iris tissues, and dominate the structure information;

- A part of iris is covered by a semitransparent layer with a bumpy look and a few furrows which are caused by retractor muscles;

- Top layer's irregular edge also contributes to the iris pattern.

- The collarette part is raised and not always at the same position as the edge of the top layer;

Thus, the main frame of the iris pattern is formed by radial fibers, raised collarette, and a partially covered semitransparent layer with an irregular edge. At the same time, the difference of pixel values in an infrared iris image is not only the result of the iris structure information. It is also related to the material that the iris is composed of, surface color, and lighting conditions.

All those common features just can make each synthetic iris looks like a real iris. To simulate the stochastic characters, this work models the generation of synthetic irises to a image processing procedure controlled by random parameters. On one hand, each parameter needs to be limited in a certain range to keep the common iris features. On the other hand, each parameter should varies as much as it can to increase the randomness of the iris pattern.

We also try to model the quality factors that affect the performance of the iris recognition system. Quality factors will change both genuine and imposter distributions, and are very 
important for testing the robustness and the performance of the system. The quality factors that are simulated in the synthesis system include occlusion, shot noise, out-of-focus blur, motion blur, rotation, contrast and lighting.

The remaining part of the chapter is organized as follows. Sec. 2.2 introduces our methodology for generating iris images. Analysis and comparison of generated iris images with real iris images is performed in Sec. 2.3 and 2.4. Three levels for comparison are identified: (1) image level, (2) texture level, and (3) decision level. While Sec. 2.3 focuses on the comparison at the first two levels, Sec. 2.4 provides detailed analysis of generated iris images at the decision level. The work concludes with a summary in Sec. 2.5.

\subsection{Methodology}

In this work, the generation of an iris image can be subdivided into five major steps:

1. Generate continuous fibers in cylindrical coordinates $(Z, R$, and $\Theta)$, where the axis $Z \in$ $(-\infty, \infty)$ is the depth of the iris, $R \in[0, \infty)$ is the radial distance, and $\Theta \in[0,2 \pi)$ is the clockwise rotational angle with a zero value corresponding to the 3 o'clock position. Each fiber is a continuous 3D curve in this cylindrical coordinate system. Presently the synthesis system uses seven random parameters, denote them as $p_{i}, i=1,2 \ldots, 7$ to generate the projection of each continuous fiber onto the two-dimensional plane $(R, \Theta)$ and six random parameters, denote them as $p_{i}, i=8,9 \ldots, 13$ to generate the projection of each fiber onto $(R, Z)$ half plane. The following equations (2.1) and (2.2) describe the dependencies of fiber coordinates in $(R, \Theta)$ and $(R, Z)$ planes:

$$
\begin{gathered}
\Theta=p_{1} \cdot \sin \left(p_{2} \cdot\left(R+p_{3}\right)\right)+p_{4} \cdot \sin \left(p_{5} \cdot\left(R+p_{6}\right)\right)+p_{7} \cdot R^{2} \\
Z=p_{8}+p_{9} \cdot \sin \left(p_{10} \cdot\left(R+p_{11}\right)\right) \cdot \sin \left(p_{12} \cdot\left(R+p_{13}\right)\right)
\end{gathered}
$$

The fiber curve is further sampled in $R$ direction to obtain matrices of $\Theta$ and $Z$ coordinates. In order to simulate petal shape patterns, another strong dependence between $\Theta$ and $Z$ was added to the fibers in pairs. 


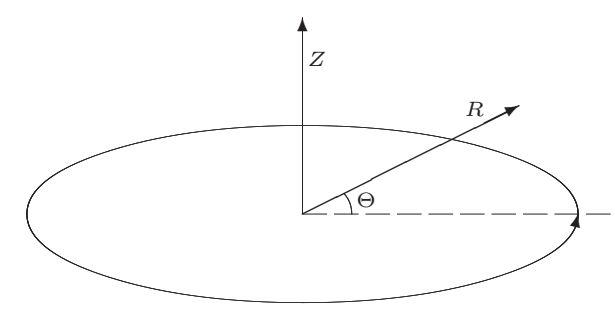

Figure 2.1: Shown is the cylindrical coordinate system.

Note that even with 13 random parameters, the generated fibers look smooth compared to real fibers. An additional option that our iris generator has is to add a "zigzag" disturbance to the original smooth functions (2.1) and (2.2). It is implemented by adding a bandwidth filtered result of a Gaussian random sequence to the original $\Theta$ and $Z$ values. Because the width of different fibers, even different parts of the same fiber, also should not be uniform, another same size matrix as $\Theta$ is added to provide width information for all sample points in $R$ direction. By this time, each fiber cannot be presented simply by several parameters anymore because of those additional totally random vibrations. It is believed that a more complex fiber model may provide a better synthetic result in terms of shape similarity and pattern randomness. But it still needs practical confirmation from anatomy aspect, such as fiber structure anatomy analysis.

2. Generated 3D fibers are then projected into a 2D polar space $(R, \Theta)$, and furthermore transformed to Cartesian pixel coordinates $(X, Y)$ forming a $2 \mathrm{D}$ frontal view fiber image. Since only top fibers can be seen in a $2 \mathrm{D}$ image, the gray value of each pixel in this $2 \mathrm{D}$ space is determined by the highest fiber at that point and equals to its $Z$ value. A set of basic B-spline functions in the polar coordinate system $(R, \Theta)$ is used to model the shapes of the pupil and the iris, that is, their deviation from a circular shape.

3. Add a top layer with an irregular edge. The edge of the top layer is modeled using the combination of cosine functions. The area where the basis images should be covered by the top layer is blurred to make the effect of a semitransparent top layer. The area of the collarette also is brightened to create the effect of a lifted portion of the iris. 


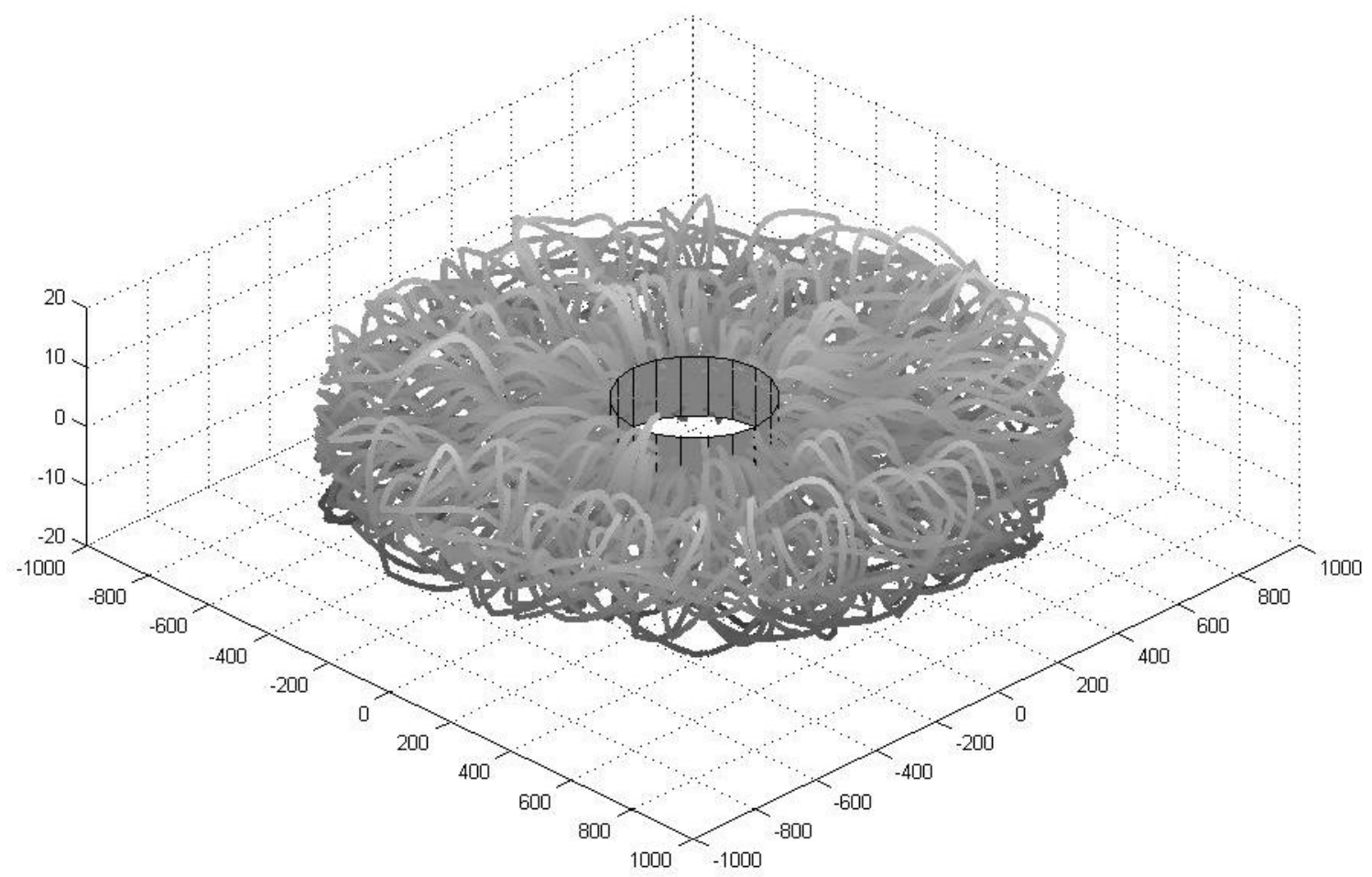

Figure 2.2: Shown is the step 1 of iris image synthesis.

4. Blur the root of the iris to make that area look continuous. Then add a smoothed Gaussian noise layer to make the top layer bumpy.

5. Based on a required degree of eyelid opening, draw two low frequency cosine curves for the eyelids. Then randomly generate the eyelashes. This step is used to simulate the occlusion which is coming from eyelids and eyelashes. Different open degrees of eyelids and different directions and amounts of eyelashes will result in different degrees of occlusion. Other simple quality factors, which effects both intra-class and inter-class distance, including shot noise (directly use a MATLAB function in the image processing toolbox), blur (simulate the out-of-focus blur or motion blur using a particular filter in MATLAB), contrast (adjust the contrast globally by compressing the range of the pixel values), specular reflections (adjust the brightness locally), can also be added in this step. More effects, such as off-angle (projective or affine transforms), can be incorporated in the final synthetic iris image. 


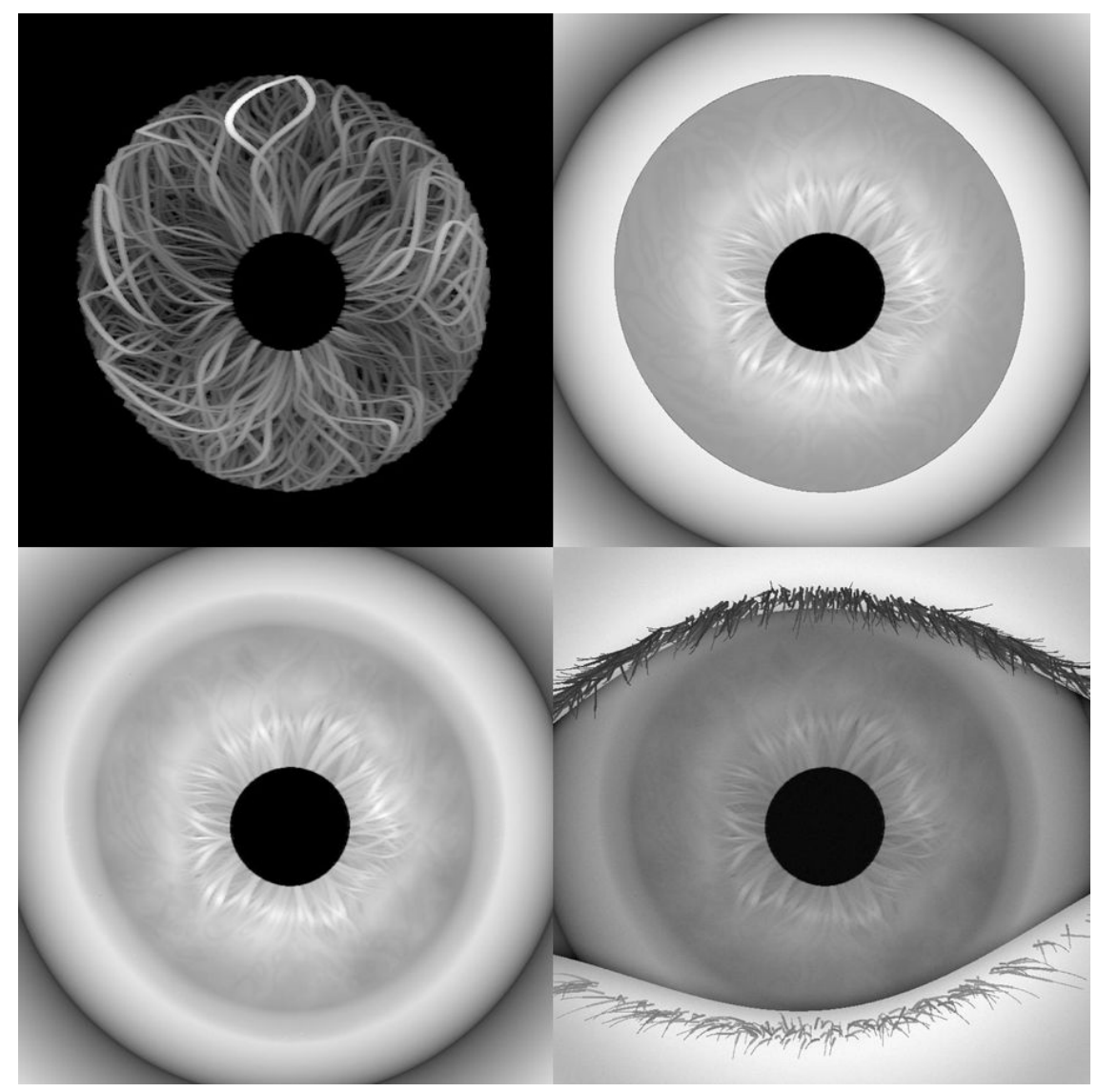

Figure 2.3: Shown are the step 2-4 of iris image synthesis. 
Fig. 2.2 and Fig. 2.3 demonstrate the whole generation procedure for a iris class. Iris images generated by the synthetic system are composed of a large number of fibers. The number may vary in the range from 500 to 2,500. This adds up to thousands of random variables that are used in the calculation of the fiber shape shown in Fig. 2.2. Besides of parameters used in the generation of fibers, the generation of the final iris image is also based on other 40 controllable random parameters including the average fiber size, the size of the pupil, the thickness of the iris, the thickness of the top layer, the cluster degree of fibers, the blur range of the iris root, the location of the collarette, the amplitude of the collarette, the span range of the collarette, the transparency parameter of the top layer, the net structure parameter, the angle and the size of two eyelids, the horizontal location of the eyeball compared to the eyelids, the number of crypts, and the direction and the amount of eyelashes. Each parameter follows uniform or Gaussian distribution on a prescribed interval. The range of each interval is tuned to ensure the appearance of synthetic irises close to the appearance of real irises.

Although every parameter has its contribution in the final result, the degrees of influence on the recognition performance are different for different parameters. To test the sensitivities of the parameters, the following experiments was conducted. All parameters were divided into three major groups: (i) parameters related to the fibers, labeled as "FIBER" (ii) parameters related to the collarette and the edge of the top layer, labeled as "COLLARETTE" and (iii) parameters responsible for other visual effects, such as blur, transparency and the bumpy texture of the top layer, labeled as "BASIC". Although the locations of the edge and the collarette are different, the parameters used to generate them are still assigned to the same group for the convenience. In the first experiment, we fixed all parameters in group (ii) and (iii) and varied parameters of the fiber structure. A set of 20 iris images was generated. Fig. 2.4 displays two sample iris images generated following this procedure. The recognition performance of those synthetic iris images was evaluated using Libor Masek's code [41] that is an implementation of the Gabor filter based method. Hamming Distance (HD) was calculated for each pair of generated iris images. The results of evaluation are shown in Fig. 2.7 (the rightmost histogram). In the second experiment, the parameters of fiber structure and the parameters in group (iii) were fixed, while the parameters that influence the generation 


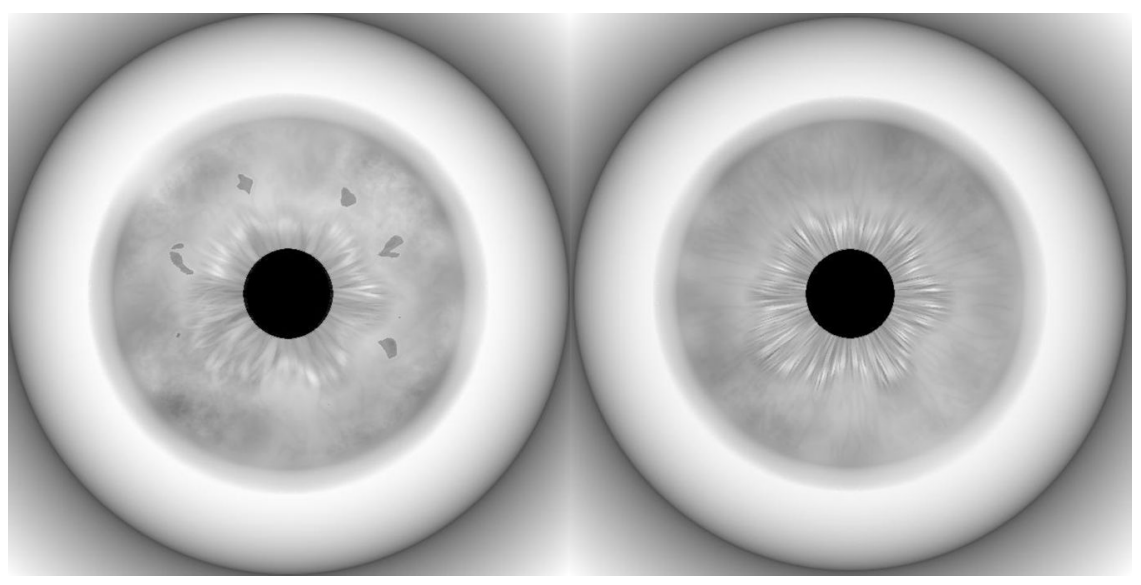

Figure 2.4: Iris images generated by varying "FIBER" parameters (crypts are associated with fibers).

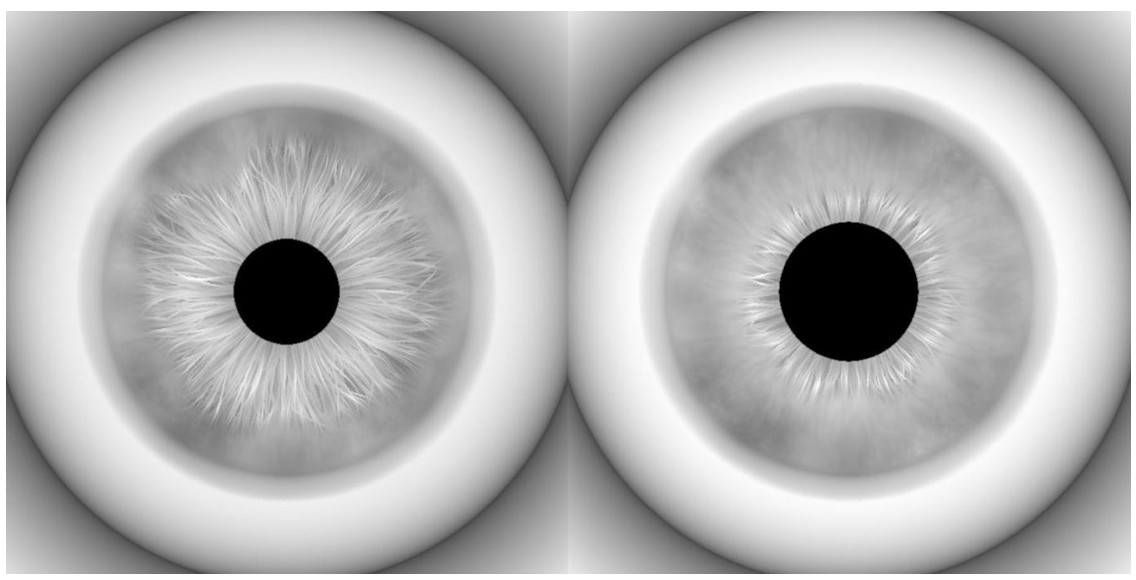

Figure 2.5: Iris images generated by varying "COLLARETTE" parameters.

of the collarette and the edge of the top layer were varied. Two sample iris images generated following this procedure are shown in Fig. 2.5. The histogram plot of HDs for this case is also displayed in Fig. 2.7 (the distribution histogram in the middle). In the last experiment, the parameters in group (i) and group (ii) were fixed, while the basic parameters were varied. A pair of generated iris images and the distribution of HDs for this case are shown in Fig. 2.6 and in Fig. 2.7 (the leftmost histogram), respectively.

From those experiments, it can be seen that the change of fibers dominate the change of synthetic iris pattern in terms of the recognition performance. The variation of the collarette and the edge of the top layer partially changes the iris pattern. However, these parameters do not influence the recognition performance as much as the fiber structure does. The external 


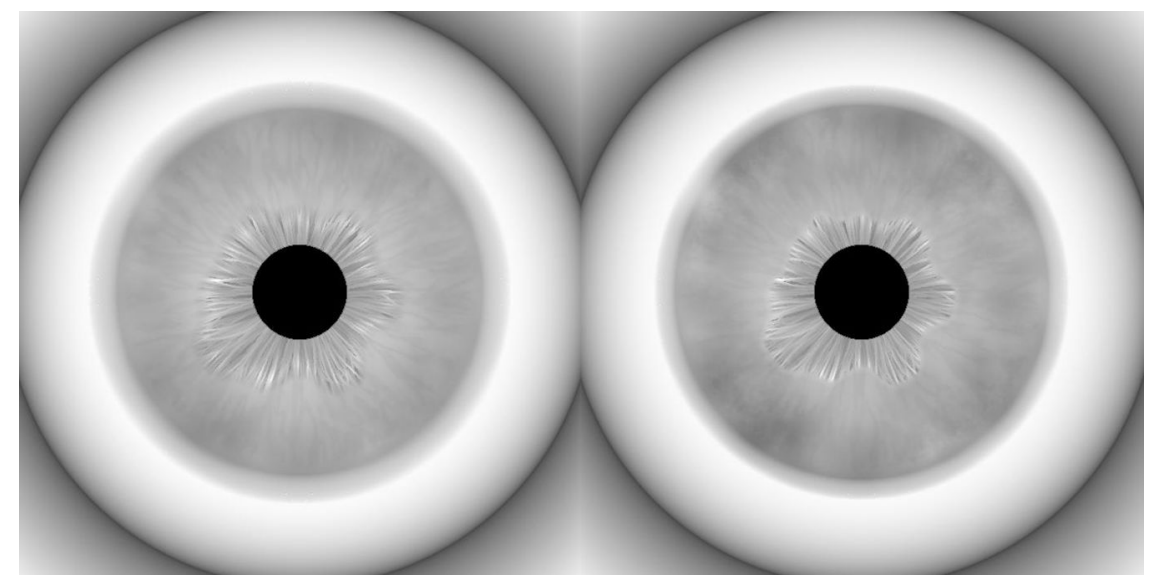

Figure 2.6: Iris images generated by varying "BASIC" parameters.

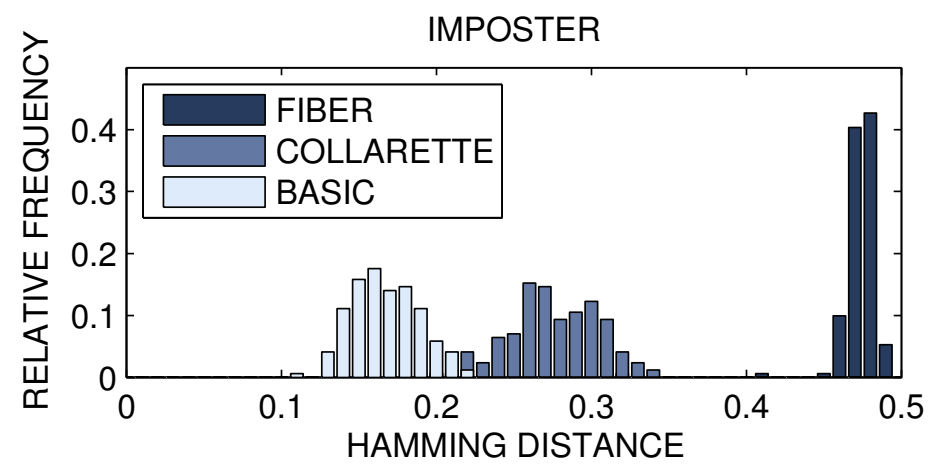

Figure 2.7: Shown are three histogram plots of HDs obtained by varying parameters in one of three major parameter groups. The rightmost histogram (marked in the dark color) was obtained when images generated by varying "FIBER" parameters were compared. The histogram in the middle (marked in the gray color) was obtained by comparing images with random "COLLARETTE" parameters. The leftmost histogram (marked in the light color) was generated by involving images generated by varying a set of "BASIC" parameters. 
effect parameters influence recognition performance the least of all parameters. In conclusion, our experimental results have shown that the recognition performance is extremely sensitive to the variation in the fiber structure and less sensitive to the variation of other parameters.

\subsection{Performance Analysis: Image and Texture Level}

Three levels are identified at which the similarity of synthetic and real iris images can be quantified. They are as follows: (i) global layout, (ii) features of fine iris texture, and (iii) recognition performance.

\subsubsection{Visual Evaluation}

A gallery of synthetic iris images generated using this anatomy based approach is shown in Fig. 2.8. To ensure that generated irises look like real irises, a few eyelids from CASIA data set were borrowed. Note that only one image in Fig. 2.8 is a real iris image, a sample from CASIA data set. It is placed among synthetic irises for the purpose of comparison. To further demonstrate that synthetic iris images look similar to real iris images, three processed iris images which come from different data sets are displayed in Fig. 2.9. They are unwrapped and enhanced to reduce the differences caused by different shapes or different degrees of contrast. The samples on the upper and middle panels are normalized images from CASIA and WVU non-ideal iris data sets. The sample on the lower panel is a normalized image from our data set of synthetic irises. Although it looks slightly over smoothed on the bottom portion of the image, the unwrapped synthetic iris image has all the major features of real iris images.

\subsubsection{Texture Analysis}

For texture analysis, features should be efficiently selected and compared. The original image is not robust enough for texture analysis. There are many filters that are suitable to extract proper features which can are used in the texture classification. But for the purpose of finding the similarity between synthetic and real irises, features can not be compared at 


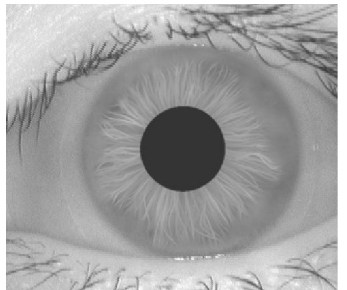

Iris 1

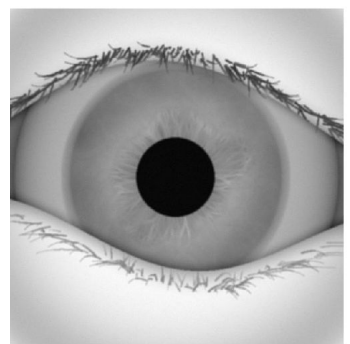

Iris 5

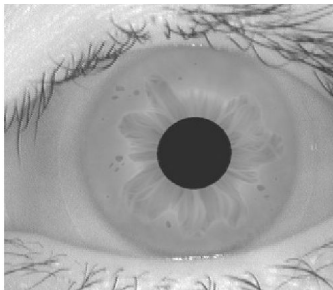

Iris 2

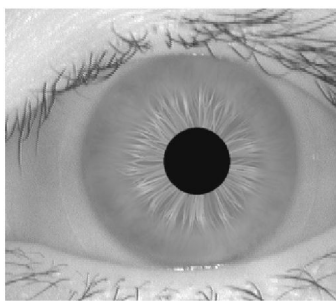

Iris 6

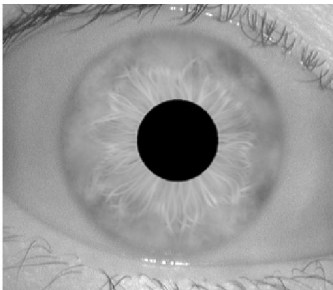

Iris 3

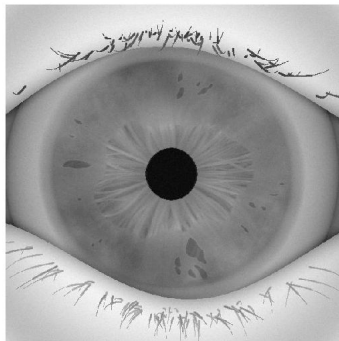

Iris 7

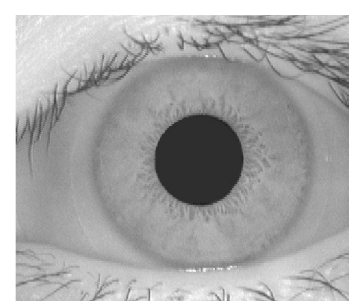

Iris 4

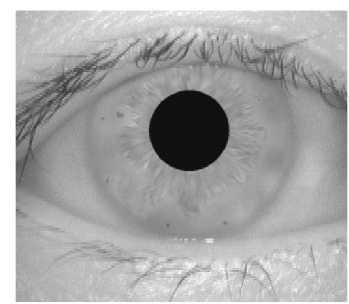

Iris 8

Figure 2.8: A gallery of synthetic iris images generated using model based, anatomy based approach. Iris 4 is a real iris image borrowed from the CASIA data set.

(a)

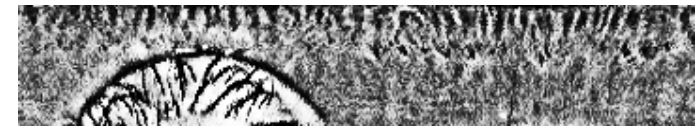

(b)

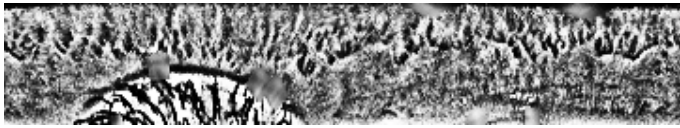

(c)

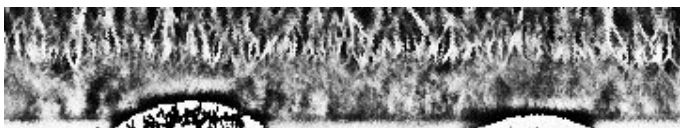

Figure 2.9: Shown are three unwrapped and enhanced iris images. The images are samples from (a) CASIA data set, (b) WVU non-ideal iris data set, and (c) data set of synthetic irises generated using our model based approach. 
the level of whole image any more. The locations of small features should be ignored. Even two images that both come from real but different iris classes, can be total different if they are compared pixel by pixel, or patch by patch. Because of this reason, the statistic model, such as the empirical distribution of the filter results, was adopted to analyze the similarity between synthetic and real irises at texture level.

Empirical probability density functions or empirical cumulative distribution functions can be directly compared. But those nonparametric methods are not very convenient to get the pairwise distance matrix. And as we know, filter results normally follow symmetric continuous distributions. If some parametric statistic models can be used to fit the distribution, then the difference between two distributions can be mapped to the difference of parameters, and even some closed forms, functions of parameters. Then the values of those close forms can be directly used in the clustering as features.

Bessel $\mathrm{K}$ form is a stochastic model that can be used to measure the image variability. As shown by Grenander and Srivastava [42], Bessel K forms parameterized by only two parameters: (1) the shape parameter $p$, and (2) scale parameter $c$, can provide good statistical fits to empirical distribution histograms of the filtered images. In this work, Bessel K forms were used to compare real and synthetic iris images against natural images.

Given an image $I$ and a filter $\mathcal{F}$, a filtered image $\mathcal{I}=I * \mathcal{F}$ is computed where $*$ denotes the 2-D convolution operation. Under the conditions stated in [42], the probability density function of the random variable $\mathcal{I}(\cdot)$ is approximated by

$$
f_{K}(x ; p, c)=\frac{1}{Z(p, c)}|x|^{p-0.5} K_{(p-0.5)}\left(\sqrt{\frac{2}{c}}|x|\right),
$$

where $K$ is the modified Bessel function, and $Z$ is the normalization given by

$$
Z(p, c)=\sqrt{\pi} \Gamma(p)(2 c)^{0.5 p+0.25} .
$$

The corresponding cumulative distribution function also can be calculated using

$$
F_{K}(x ; p, c)=\int_{-\infty}^{x} f_{K}(r ; p, c) d r .
$$

Then each image $I$ can be mapped to a point in the probability density space

$$
\mathcal{D}=\left\{f_{K}(x ; p, c) \mid p>0, c>0\right\} .
$$



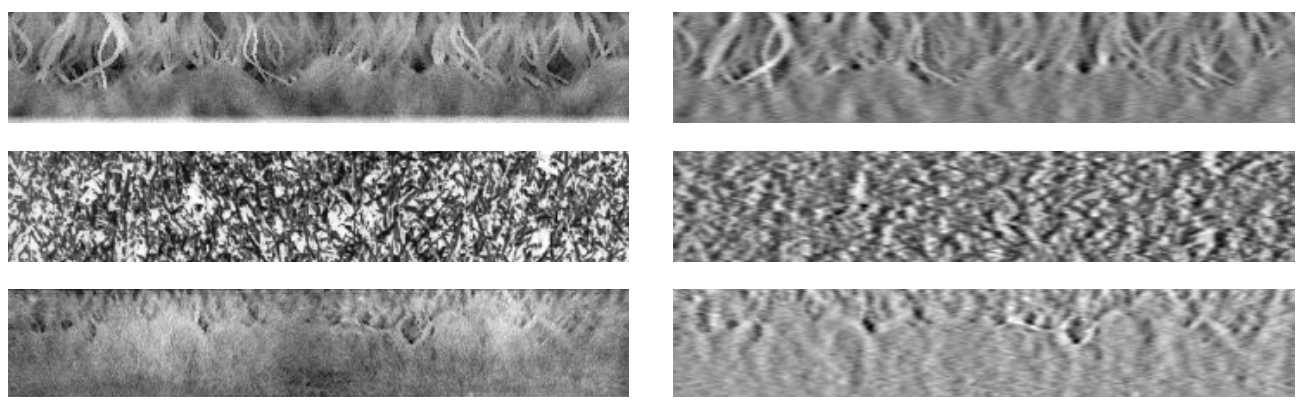

Figure 2.10: Original images (left column) and their real components of log-Gabor filtered results (right column).

The parameters $p$ and $c$ can be estimated from the observed data using

$$
\hat{p}=3 /(S K(\mathcal{I})-3) \text { and } \hat{c}=S V(\mathcal{I}) / \hat{p}
$$

where $S K$ is the sample kurtosis and $S V$ is the sample variance of the pixel values in $\mathcal{I}$. Since the moment-based estimator is suspect to outliers, observed quartiles can be used to estimate $p$ by

$$
\hat{p}=3 /(\hat{S K}(\mathcal{I})-3),
$$

where

$$
\hat{S K}(\mathcal{I})=\frac{q_{0.995}(\mathcal{I})-q_{0.005}(\mathcal{I})}{q_{0.75}(\mathcal{I})-q_{0.25}(\mathcal{I})}
$$

and $q_{x}(\cdot)$ is the quartile function that returns the $x$ quartile of a set of samples.

A normalized iris image from synthetic database, a natural image [43] and a normalized iris image from CASIA database are displayed in Fig. 2.10 left column. They were filtered using a log-Gabor filter designed by Masek [41]. The real components of filtered images are shown in the right column of Fig. 2.10. The distribution histograms and estimated Bessel K forms of these filtered images are displayed in Fig. 2.11. The real and imaginary components of each filtered image were mixed to get a single Bessel $\mathrm{K}$ form fit.

To compare real, synthetic iris images and natural images [43], initially 12 images were selected from each group. Table 2.2 presents a summary of the estimated parameters of Bessel $\mathrm{K}$ forms for all images that were filtered by log-Garbor filters. For parameter $p$, the range of values in the case of synthetic data set is similar to that in the case of CASIA data set, while is quite different from that in the case of natural images. 

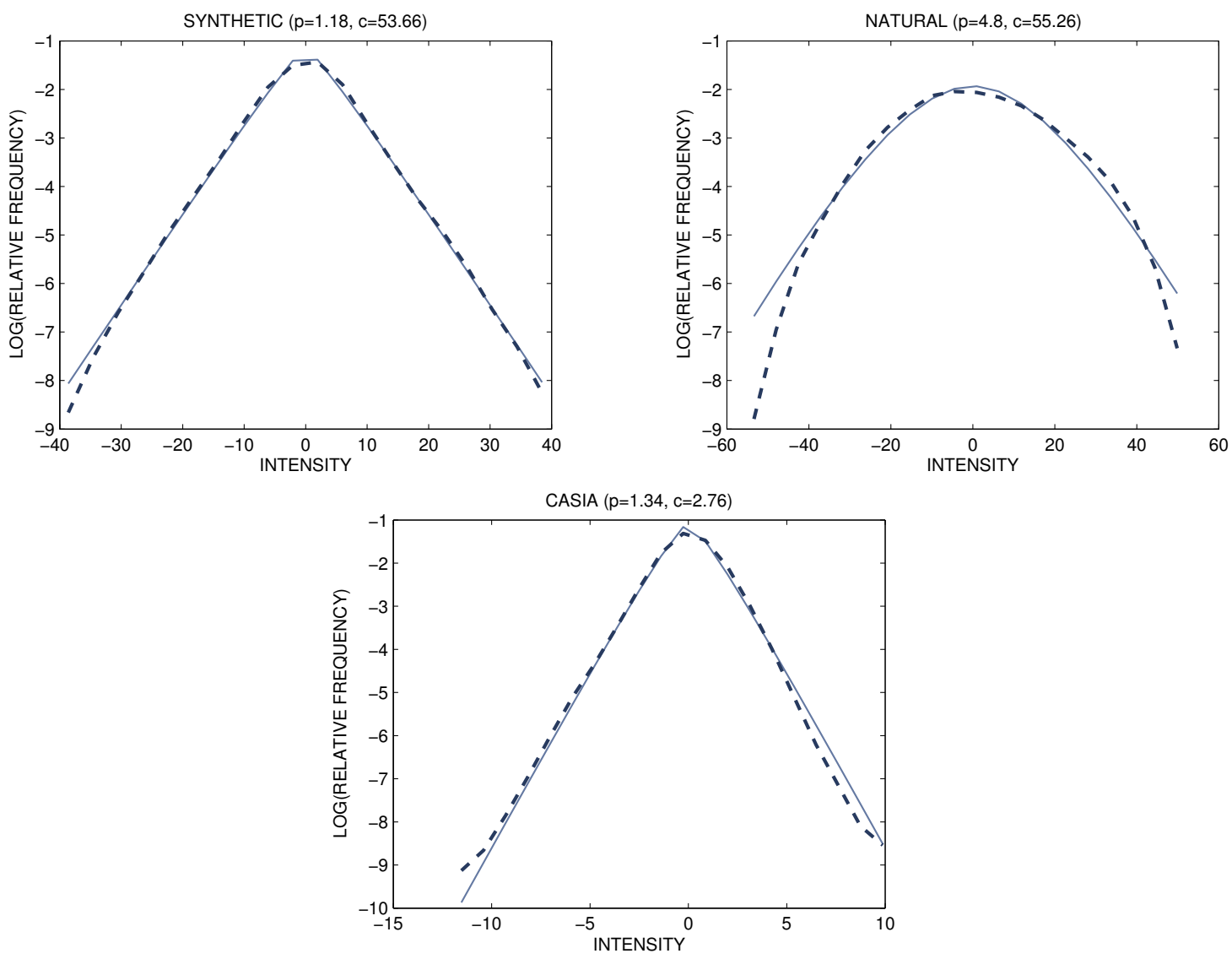

Figure 2.11: The empirical histogram distributions (dashed lines) and their Bessel K form approximations (solid lines). 
Table 2.2: The Bessel K parameters estimated from images filtered by log-Gabor filter

\begin{tabular}{|l|c|r||l|c|c||l|r|r|}
\hline Image & $\mathrm{p}$ & \multicolumn{1}{c||c}{} & Image & $\mathrm{p}$ & $\mathrm{c}$ & Image & $\mathrm{p}$ & \multicolumn{1}{c|}{$\mathrm{c}$} \\
\hline CA1 & 1.40 & 2.43 & SYN1 & 1.40 & 25.89 & NA1 & 0.52 & 226.12 \\
\hline CA2 & 1.17 & 2.23 & SYN2 & 0.88 & 24.77 & NA2 & 2.16 & 79.66 \\
\hline CA3 & 0.64 & 13.80 & SYN3 & 1.30 & 34.30 & NA3 & 15.47 & 22.85 \\
\hline CA4 & 1.32 & 3.21 & SYN4 & 1.05 & 29.07 & NA4 & 8.04 & 48.85 \\
\hline CA5 & 0.79 & 9.63 & SYN5 & 0.51 & 38.05 & NA5 & 2.20 & 122.42 \\
\hline CA6 & 0.82 & 4.84 & SYN6 & 0.70 & 33.57 & NA6 & 3.19 & 38.22 \\
\hline CA7 & 0.53 & 20.80 & SYN7 & 1.09 & 25.99 & NA7 & 1.54 & 159.39 \\
\hline CA8 & 1.07 & 2.58 & SYN8 & 0.65 & 46.46 & NA8 & 0.93 & 30.24 \\
\hline CA9 & 0.91 & 7.92 & SYN9 & 1.04 & 40.03 & NA9 & 4.80 & 55.26 \\
\hline CA10 & 0.99 & 3.78 & SYN10 & 1.69 & 13.68 & NA10 & 0.86 & 247.17 \\
\hline CA11 & 1.34 & 2.76 & SYN11 & 0.80 & 22.93 & NA11 & 8.51 & 33.68 \\
\hline CA12 & 0.91 & 3.54 & SYN12 & 1.18 & 53.66 & NA12 & 4.84 & 96.66 \\
\hline
\end{tabular}

To quantify the difference between two images according to their distributions of the filtered results, Three kinds of distances, (1) a pseudo-metric introduced by Srivastava [42], (2) the k-measure, between two Bessel K forms were used and (3) the integral of the difference between two cumulative distribution functions. The first one is given by

$$
d_{I}\left(I_{1}, I_{2}\right)=d\left(\mathcal{I}_{1}, \mathcal{I}_{2}\right)=\sqrt{\int_{-\infty}^{\infty}\left(f_{K}\left(x ; p_{1}, c_{1}\right)-f_{K}\left(x ; p_{2}, c_{2}\right)\right)^{2} d x}
$$

The second distance is given by

$$
d_{K L}\left(I_{1}, I_{2}\right)=D\left(f_{K}\left(x ; p_{1}, c_{1}\right) \| f_{K}\left(x ; p_{2}, c_{2}\right)\right)+D\left(f_{K}\left(x ; p_{2}, c_{2}\right) \| f_{K}\left(x ; p_{1}, c_{1}\right)\right),
$$

where

$$
D\left(f_{K}\left(x ; p_{1}, c_{1}\right) \| f_{K}\left(x ; p_{2}, c_{2}\right)\right)=\int_{-\infty}^{\infty} \log \left(\frac{f_{K}\left(x ; p_{1}, c_{1}\right)}{f_{K}\left(x ; p_{2}, c_{2}\right)}\right) f_{K}\left(x ; p_{1}, c_{1}\right) d x .
$$

And the last distance is given by

$$
d_{C D F}\left(I_{1}, I_{2}\right)=\int_{-\infty}^{\infty}\left|F_{K}\left(x ; p_{1}, c_{1}\right)-F_{K}\left(x ; p_{2}, c_{2}\right)\right| d x
$$

$f_{K}(\cdot)$ used in (2.5), (2.6) and (2.7) is the Bessel K probability density function introduced in (2.3). And $F_{K}(\cdot)$ used in (2.8) is the Bessel $\mathrm{K}$ cumulative distribution function introduced in $(2.4)$.

For $N$ images $\left\{I_{n}, n=1,2, \ldots N\right\}$ and a bank of filters $\left\{\mathcal{F}_{j}, j=1,2, \ldots J\right\}$, a set of filtered images $\left\{\mathcal{I}_{(n, j)}=I_{n} * \mathcal{F}_{j}, j=1,2, \ldots J\right\}$ are computed. And after estimating the parameter 
$p_{(n, j)}$ and $c_{(n, j)}$, each image is mapped to $J$ points in the density space. The corresponding distances also need to be changed from (2.5), (2.6) and (2.8) to

$$
\begin{gathered}
d_{I}\left(I_{1}, I_{2}\right)=\sum_{j=1}^{J} d\left(\mathcal{I}_{(1, j)}, \mathcal{I}_{(2, j)}\right), \\
d_{K L}\left(I_{1}, I_{2}\right)=\sum_{j=1}^{J}\left[D\left(f_{K(1, j)}|| f_{K(2, j)}\right)+D\left(f_{K(2, j)}|| f_{K(1, j)}\right)\right],
\end{gathered}
$$

and

$$
d_{C D F}\left(I_{1}, I_{2}\right)=\sum_{j=1}^{J} \int_{-\infty}^{\infty}\left|F_{K}\left(x ; p_{(1, j)}, c_{(1, j)}\right)-F_{K}\left(x ; p_{(2, j)}, c_{(2, j)}\right)\right| d x .
$$

where

$$
f_{K(n, j)}=f_{K}\left(x ; p_{(n, j)}, c_{(n, j)}\right),
$$

and

$$
F_{K(n, j)}=F_{K}\left(x ; p_{(n, j)}, c_{(n, j)}\right),
$$

Distances $d_{I}$ also can be calculated by

$$
d_{I}\left(I_{1}, I_{2}\right)=\sqrt{\left(\sum_{j=1}^{J}\left[d\left(\mathcal{I}_{(1, j)}, \mathcal{I}_{(2, j)}\right)\right]^{2}\right)} .
$$

Thus the entire procedure resulting in classification of texture images can be subdivided into five steps:

1. Convolve each image $I_{n}$ with each filter $\mathcal{F}_{j}$ included in the Filter Bank.

2. Estimate the parameters of Bessel $\mathrm{K}$ forms for each filtered image $\left\{\left(\hat{p}_{(n, j)}, \hat{c}_{(n, j)}\right), n=\right.$ $1,2, \ldots N, j=1,2, \ldots J\}$.

3. Drop the filters which resulted in a estimated values $\hat{p}_{(n, j)}<0.25$ (see [42] for details).

4. Calculate pairwise distance matrices $d_{I}, d_{K L}$ and $d_{C D F}$.

5. Use the distance matrices to perform the hierarchical clustering of the filtered images. 

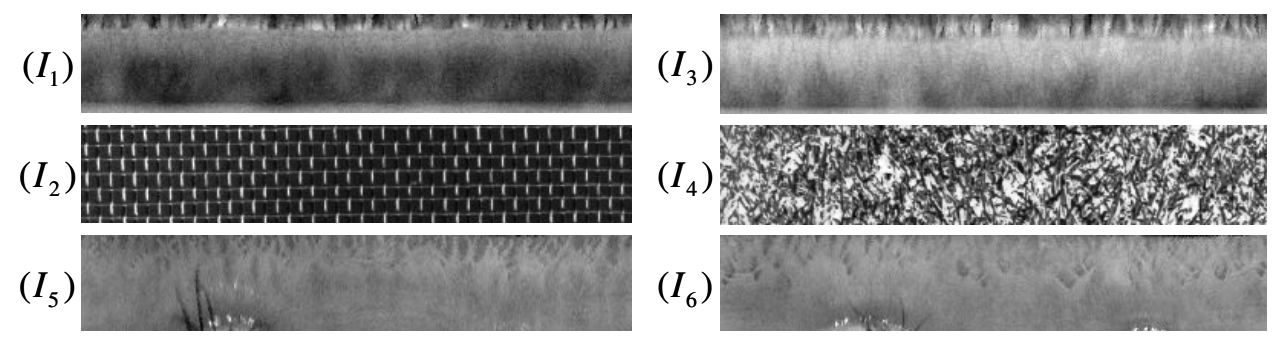

Figure 2.12: Original images. $I_{1}$ and $I_{2}$ are synthetic iris images, $I_{3}$ and $I_{4}$ are natural images and $I_{5}$ and $I_{6}$ are real iris images.
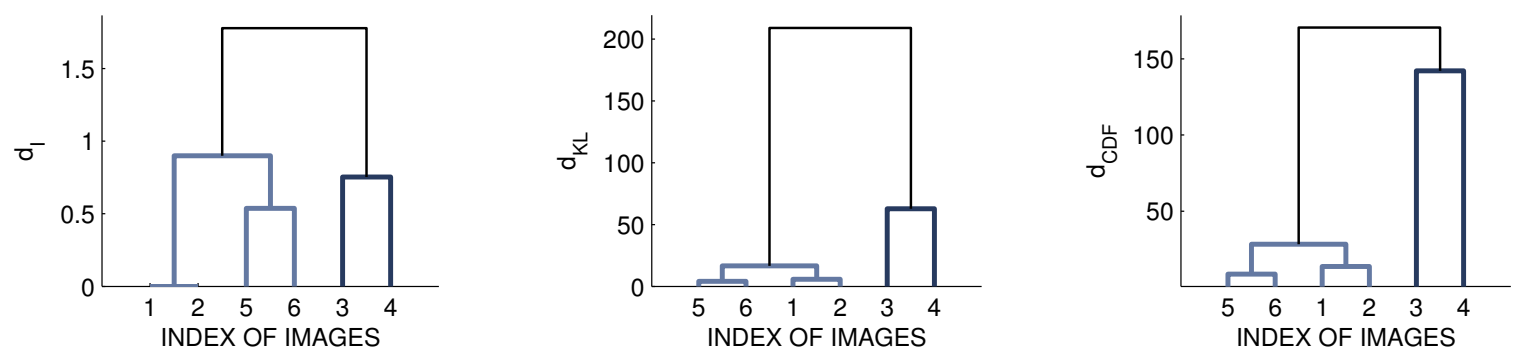

Figure 2.13: The left and the right panels show the dendrogram clustering plot using the distance matrices $d_{I}$ and $d_{K L}$, respectively.

For the purpose of texture analysis using different features, the log-Gabor filter used by Libor Masek [41], the Leung-Malik (LM) Filter Bank [44], the Maximum Response (MR) Filter Bank [44] and the Schmid (S) Filter Bank [44] were used respectively in the experiment. And 12 iris images from CASIA data set which have the least occlusion, total 111 natural texture images from Brodatz textures database [43] and 30 synthetic iris images were selected as the samples in the experiment.

To show the clear hierarchical clustering result, two images from each group were selected. Fig. 2.12 shows all 6 images. Normalized iris images $I_{1}$ and $I_{2}$ were selected from the data set of synthetic iris images. Images $I_{3}$ and $I_{4}$ were selected from the set of natural images and cropped to the same size as unwrapped iris images. And normalized iris images $I_{5}$ and $I_{6}$ were selected from CASIA data set. The left and the right panels in Fig. 2.13 display two dendrogram plots that summarize the clustering results based on the matrices $d_{I}, d_{K L}$ and $d_{C D F}$. Images are filtered by 42 valid filters selected from the LM Filter Bank.

Fig. 2.14 shows the clustering results using all 153 images with 4 different filter or filter banks. For the convenience, two classes, "IRIS" and "NON-IRIS", are used. The maximum 


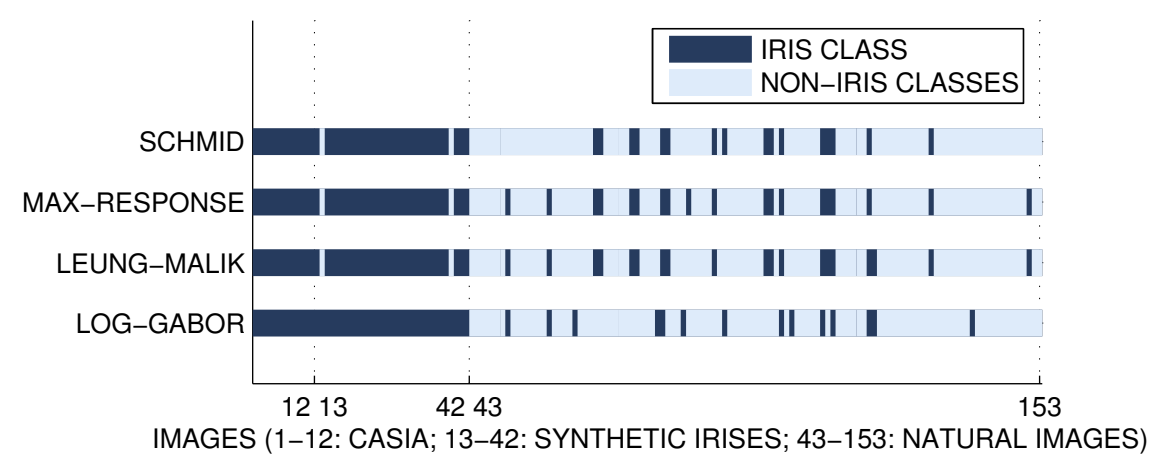

Figure 2.14: The clustering results for all 153 images and 4 different filter or filter banks.

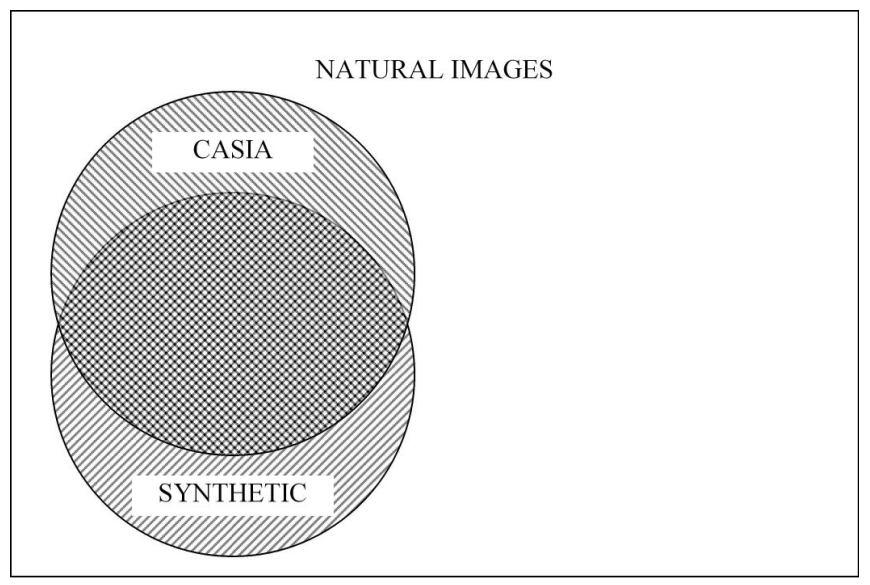

Figure 2.15: The relationships between different groups of images based on the texture analysis.

number of clusters was adjusted to make all CASIA images and most synthetic iris images clustered to the same "IRIS" class. From this graph, it can be seen that only a few of natural images, are clustered to the same class as CASIA real iris images. Compared with the large variety of the natural images, Both real irises and synthetic irises shows limited varieties, and they have a certain degree of overlap which varies a little bit on the different features extracted using different filters. The relationship between them can be explained by Fig. 2.15 . 


\subsection{Performance Analysis: Decision Level}

\subsubsection{Verification Performance}

For biometrics system, the most important statistic character is the identification or verification performance. To evaluate the performance of synthetic iris images from the recognition perspective, the Gabor filter based encoding technique by Libor Masek [41] [45] were used. The synthetic iris images characterizing 200 individuals, 2 iris classes per individual, 6 iris images per iris class including an ideal image (no effects added), a noisy image with additive shot noise, a rotated image (to simulate the roll movement), a out-offocus image, a smeared image (motion blur) and a low contrast image were generated. Those images are shown in Fig. 2.16 in the order from left to right and from top to bottom. The left panel in Fig. 2.17 shows the histograms of genuine and imposter HD distributions for the generated data. The d-prime $\left(d^{\prime}\right)$, a measure of separation between genuine and imposter matching score distributions (see [46] [8] for definition), is equal to 6.7493. The right panel in Fig. 2.17 shows the corresponding Receiver Operating Characteristic (ROC) curve. Note that both histogram plots and the ROC curve follow traditional shapes for iris recognition.

\subsubsection{Analysis of Degrees of Freedom}

False Accept Rate (FAR), one of the most important indicators of the security level for biometrics systems, is completely determined by the imposter distribution. In this and the following section the distributions of imposter HDs for synthesized and real iris data are analyzed.

In his work Daugman suggested to use a Binomial distribution as the best fit of empirical imposter data [46] [8], even when the bits in the binary iris code are not independent. The Binomial distribution of HDs is parameterized by the probability for each independent bit to be $1 p$ and the degrees of freedom $N$, and is given by the probability of each possible value $k$

$$
P(k)=\frac{N !}{k !(N-k) !} p^{k}(1-p)^{N-k}, k=0,2 \ldots, N .
$$




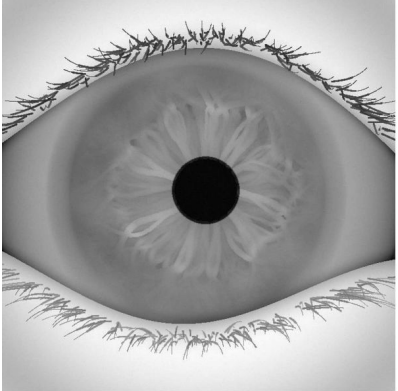

(1)

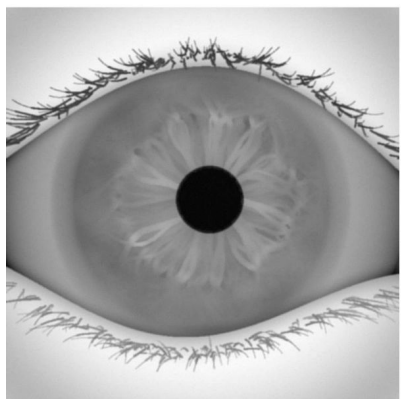

(4)

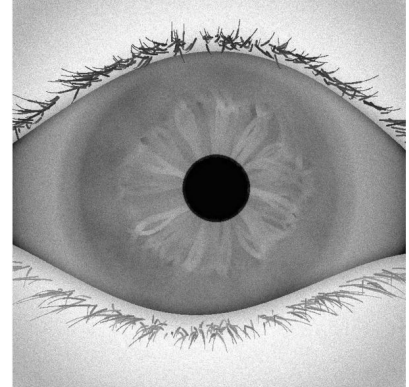

(2)

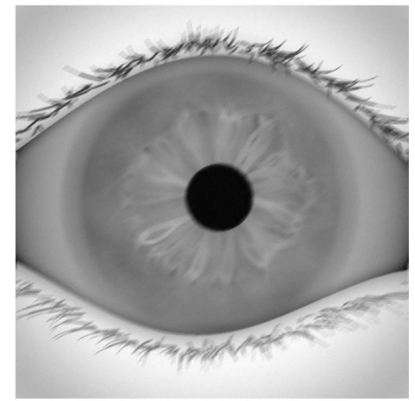

(5)

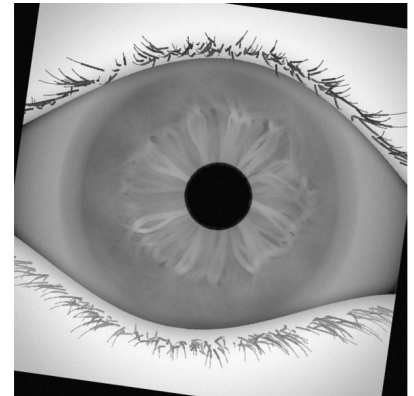

(3)

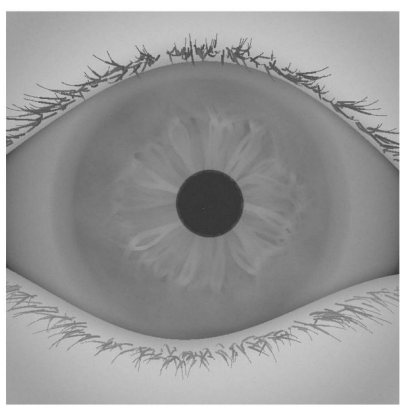

(6)

Figure 2.16: The original image and the other results when different quality factors are added (1: the original image; 2: shot noise; 3: rotation; 4:out-of-focus blur; 5:motion blur; 6:low contrast).
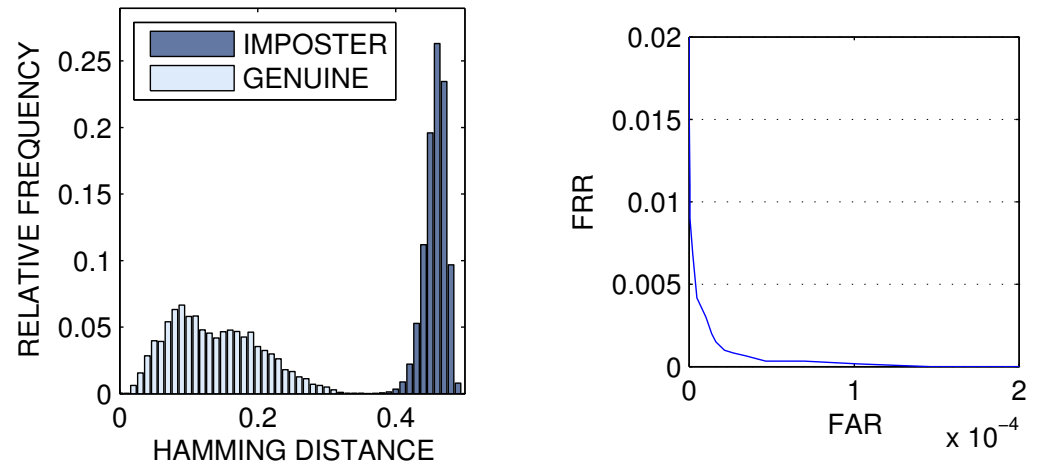

Figure 2.17: The left panel shows the histograms of genuine and imposter HDs characterizing the performance of synthetic iris images processed using the recognition system implemented by Libor Masek. The right panel shows the corresponding ROC curve. 
If it is expressed in the format of stair curve, the function changes to

$$
f_{B}(n)=P(\lfloor n+0.5\rfloor), n \in[-0.5, N+0.5]
$$

The normalized HDs follow the compressed distribution

$$
f_{0}(x)=N \times f_{B}(x \times N), x \in\left[-\frac{1}{2 N}, 1+\frac{1}{2 N}\right],
$$

with the mean $p$ and the variance $p(1-p) / N$. Scale $N$ is added to keep the integral equals to 1. In this case, as has been shown in [8], resulting Binomial distribution has reduced degrees of freedom. Here the same strategy and Binomial distribution were adopted to analyze the difference between synthetic and real iris data. Since the details of Daugman's system implementation are not available (the results of analysis are presented in [46] [8]), Masek's approach [41] [45] were used to processing iris image data. When the normalized templates of size $20 \times 240$ were used, each iris code has 9600 bits in total. No compensation for rotation (the registration of two iris codes) is performed when the distribution of the HDs is evaluated. The estimation of the degrees of freedom $\hat{N}$ were calculated using

$$
\hat{N}=\frac{\hat{p}(1-\hat{p})}{\hat{\sigma}^{2}},
$$

where $\hat{p}$ and $\hat{\sigma}^{2}$ were the sample mean and the sample variance for each set of imposter scores obtained from synthetic or real iris data. Fig. 2.18 shows the distribution of HDs for imposter scores obtained from synthetic data and its best Binomial distribution fit. The estimated value of degrees of freedom for synthetic irises is 376 .

The degrees of freedom for CASIA data set were further estimated using the same template size and the same Gabor filter. CASIA data set yielded 507 degrees of freedom. Note a very high number of degrees of freedom in templates from CASIA data set in this case. This phenomenon that CASIA data set has a higher degrees of freedom can be best explained by the presence of fine textures in the images from CASIA data set. Maybe it also partially caused by the shot noise. Those fine textures add more randomness of bits for the large size templates. Conversely, for the smaller template size, the effect of fine texture is removed due to averaging over large image patches. The major role of the randomness was assigned to the medium or large size features in this case. It appears that CASIA data set does not 


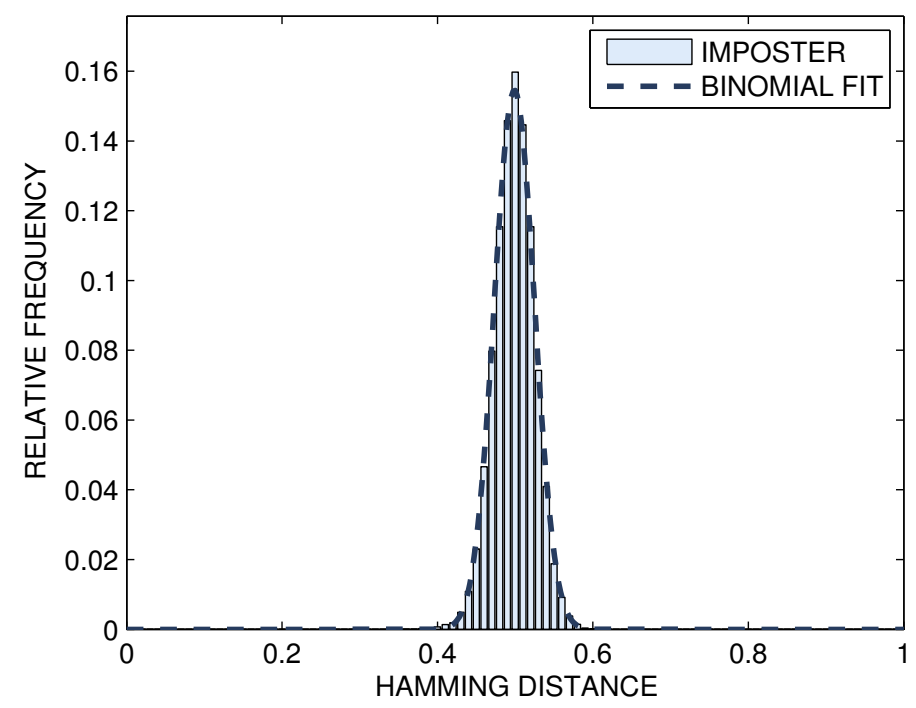

Figure 2.18: Shown are the empirical distribution of imposter HDs and the Binomial curve $(N=376)$ that provides the best fit. The HD scores were obtained without performing compensation for rotation.

have as many large features as the images in synthetic data set. The results of our analysis is summarized in Table 2.3.

Table 2.3: Degree of freedom

\begin{tabular}{|c|c|c|}
\hline Template Size & Synthetic Data Set & CASIA Data Set \\
\hline 8-by-128 & 187 & 128 \\
\hline 16-by-90 & 143 & 65 \\
\hline 32 -by-180 & 319 & 348 \\
\hline 48 -by-270 & 608 & 823 \\
\hline 64-by-360 & 1154 & 1391 \\
\hline 20 -by-240 & 376 & 507 \\
\hline
\end{tabular}

\subsubsection{Performance Extrapolation}

In this section, tails of imposter distributions is modeled using the theory of extremes, and the similarity in the behavior of extrapolated tails for synthetic and real iris data in a large scale identification system is demonstrated. As stated in Sec. 2.4.2, FAR is one of the most important characterizations of an iris recognition system. In traditional iris recognition systems the decision about the natures of two iris images is made based on 
comparing a test statistic (for example, a distance measured between two iris images) with a threshold, where the threshold has to be selected such that it satisfies designed security requirements. In Daugman's system the normalized HD, denoted by $D_{H}\left(C_{1}, I_{2}\right)$, between two binary iris codes $C_{1}$ and $C_{2}$ plays the role of the test statistic. Then the decision rule is given by

$$
\begin{aligned}
& \text { if }\left(D_{H}\left(C_{1}, C_{2}\right)>\gamma\right) \text {, decide Imposter, } \\
& \text { if }\left(D_{H}\left(C_{1}, C_{2}\right)<\gamma\right) \text {, decide Genuine, }
\end{aligned}
$$

where $\gamma$ is a threshold. To obtain the complete characterization of the designed system, FAR and False Rejection Rate (FRR) have to be evaluated for each value of $\gamma$ on the interval between two empirical means of the test statistics, the empirical mean under the Genuine assumption and the empirical mean under the Imposter assumption. However, if the purpose of performance evaluation is to estimate the probability of error and predict the error in a large scale identification system, the focus has to be placed only on the tails of Genuine and Imposter distributions. In terms of thresholds, it is a relatively narrow area. The idea of performance extrapolation based on tail model has been previously studied. For example, in his recent work [8] Daugman used Binomial Minimum Value distribution to fit the angle compensated imposter curve, and predicted the performance of identification system. Considering a simple verification scenario a one to one False Accept Rate $F A R_{1: 1}$ is gotten. For identification system with $M$ independent templates in the database, the expression for the total False Accept Rate $F A R_{1: M}$ is given by

$$
F_{1: M}(x)=1-\left[1-F_{1: 1}(x)\right]^{M},
$$

if each distinct comparison introduces a identical independent sample.

The rest of this section is organized as follows. In the first part of this section, a strategy similar to Daugman's was adopted for performance prediction of iris based identification systems and used the results of extrapolation as a measure of "realism" in comparing synthetic and real iris data. In the second part of the section, the theory of extremes was invoked. 


\section{Binomial Minimum Value Analysis}

From the compressed Binomial density function $f_{0}(x)$ which fitted into the empirical imposter distribution best under the condition that no rotation was compensated, the compressed Binomial cumulative distribution function $F_{0}(x)$ can be calculated using

$$
F_{0}(x)=\int_{0}^{x} f_{0}(r) d r
$$

where $f_{0}(x)$ is introduced in (2.9). If it is assumed that each rotation compensation will introduce an independent testament, the minimum value of the HDs will follow the distribution

$$
F_{m}(x)=1-\left[1-F_{0}(x)\right]^{m},
$$

which is called Binomial Minimum Value distribution [8]. In verification scenario, False Accept Rate $F A R_{1: 1}$ will be estimated using $F_{m}(x)$.

All normalized images in our experiments were transformed to have the same template size 20-by-240. Masek's code was further used to generate binary iris codes and calculate the imposter HDs. In our experiments, for each pair of iris templates, an alignment was performed by fixing one of them and shifting the other one in the range from -20 pixels to 20 pixels (corresponds to the rotation of the original iris image in the range of angles from -30 degrees to 30 degrees provided that the templates of size 20-by-240 are used). It is assumed that minimum HDs obtained using distinct pairs can be treated as independent and identically distributed in (2.12). To find the Binomial Minimum Value distribution that best fits an imposter distribution, two parameters, the degrees of freedom $N$ and the degrees of rotation compensation $m$, were adjusted, while another parameter the mean $p$ was fixed on 0.5 .

The empirical imposter distribution of HDs for synthetic data is formed using 2,872,800 imposter HD scores which come from 200 users, 2 classes per user and 6 images per class. The left panel in Fig. 2.19 shows the log scale plot of the Binomial Minimum Value distribution parameterized by $N=373$ and $m=11$ that provided the best fit to the imposter HDs obtained using synthetic data. The right panel in Fig. 2.19 shows the same plot but in linear scale.

For CASIA database, the Binomial Minimum Value distribution that provided the best fit to the empirical imposter HDs is described by $N=437$ and $m=14$, while for synthetic iris 

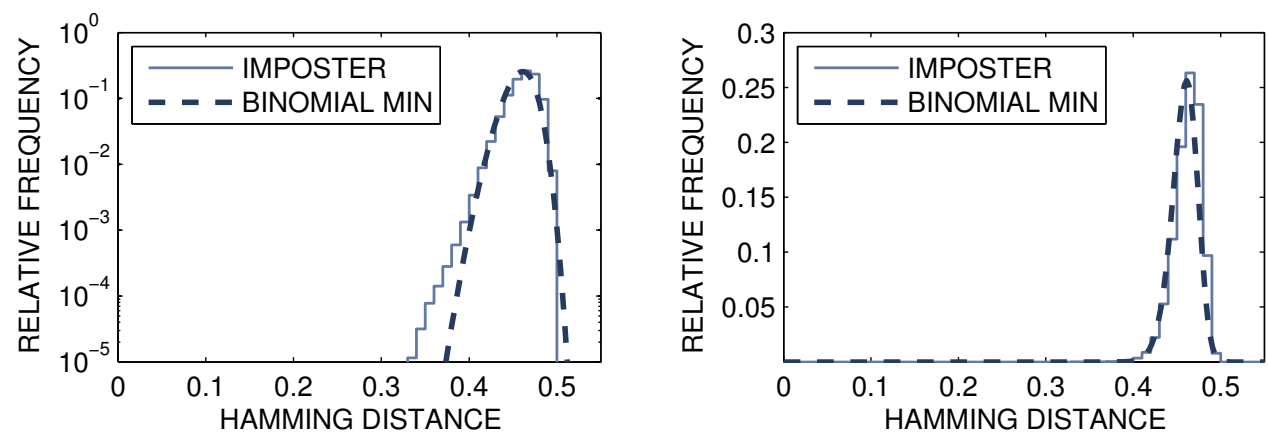

Figure 2.19: The left panel shows the log scale Binomial Minimum Value density function that provided the best fit to the distribution of imposter HDs generated from synthetic data. The right panel compares the Binomial Minimum Value density function and the histogram distribution of HDs obtained from synthetic data. The temples used to perform experiments are of size 20 -by-240.

with the same sample size, the distribution is described by $N=381$ and $m=11$. To obtain these results, 108 classes, 6 images per class from CASIA and synthetic iris data set were used. And this resulted in 208008 imposter comparisons for both of them. For synthetic data, first 208008 from total 2,872,800 imposter comparisons were directly selected. This subset of full data also resulted in a different parameter estimation as shown above. Table 2.4 summarizes the predicted results for the database size $M$ in the range from $10^{3}$ to $10^{8}$. The second column in this table displays the fixed values of $F A R_{1: M}$ for identification case. The third column displays the values of the corresponding $F A R_{1: 1}$ in verification case. The columns 4 and 5 show the extrapolated values of thresholds $\gamma$ obtained using templates of size 20-by-240 obtained using CASIA and Synthetic iris data set that resulted in $1 \% F A R_{1: M}$ for identification case. Using Binomial Minimum Value distribution, the threshold $\gamma$ can be directly calculated from $F A R_{1: 1}$ using

$$
\gamma=F_{m}^{-1}\left(F A R_{1: 1}\right)
$$

where $F_{m}(\cdot)$ is introduced in $(2.12)$.

\section{Extreme Value Analysis}

The results in Table 2.4 look encouraging. However, a careful analysis of the results indicates that the data do not exactly follow the original Binomial Minimum Value distribution. The assumption that different relative orientations used in the angle compensation 
Table 2.4: The threshold values and its effect on FAR. The results are obtained using Binomial Minimum Value distribution (with 208008 imposter comparisons)

\begin{tabular}{|c|c|c|c|c|}
\hline Database & Estimated & Estimated & \multicolumn{2}{|c|}{ HD Threshold $\gamma$} \\
\cline { 4 - 5 } Size $(M)$ & $F A R_{1: M}$ & $F A R_{1: 1}$ & CASIA & Synthetic \\
\hline $10^{3}$ & $1.0 \%$ & $1.0050 \times 10^{-5}$ & 0.3844 & 0.3780 \\
\hline $10^{4}$ & $1.0 \%$ & $1.0050 \times 10^{-6}$ & 0.3753 & 0.3675 \\
\hline $10^{5}$ & $1.0 \%$ & $1.0050 \times 10^{-7}$ & 0.3661 & 0.3570 \\
\hline $10^{6}$ & $1.0 \%$ & $1.0050 \times 10^{-8}$ & 0.3570 & 0.3465 \\
\hline $10^{7}$ & $1.0 \%$ & $1.0050 \times 10^{-9}$ & 0.3478 & 0.3386 \\
\hline $10^{8}$ & $1.0 \%$ & $1.0050 \times 10^{-10}$ & 0.3410 & 0.3307 \\
\hline
\end{tabular}

are independent does not hold in practice. In fact, as seen in Sec. 2.4.3, a larger number (almost doubled) of different relative rotation compensations were use to make sure finding of the best match. Different degrees of occlusion also add some uncertain factors to the normalization procedure of HDs. To obtain a better approximation of tails in the imposter distributions, we appeal to the extrapolation method based on extreme values. This method is often used to estimate tails of distributions from a few observed values. The theory of extreme values assumes that the tail distribution can be approximated by an empirical distribution obtained from independent tail (the minimum value) samples. This distribution is further approximated by a parametric extreme value distribution. The parameters of the extreme value distribution that provide the best fit into empirical data are obtained using the Maximum Likelihood (ML) estimation procedure.

The Type 1 (Gumbel) extreme value distribution [47] was used to approximate the empirical distribution. A Gumbel extreme value distribution is parameterized by two parameters, the location parameter $\mu$ and the scale parameter $\sigma$, and is given by

$$
f_{E}(x: \mu, \sigma)=\sigma^{-1} \exp \left(\frac{x-\mu}{\sigma}\right) \exp \left(-\exp \left(\frac{x-\mu}{\sigma}\right)\right) .
$$

Its corresponding cumulative distribution function is given by

$$
F_{E}(x: \mu, \sigma)=\int_{\infty}^{x} f_{E}(r: \mu, \sigma) d r,
$$

and used to estimate the False Accept Rate $F A R_{1: 1}$.

To find the distribution function that provides the best fit, the Maximum Likelihood estimates of the parameters $\mu$ and $\sigma$ were found by invoking the function "evfit" from 

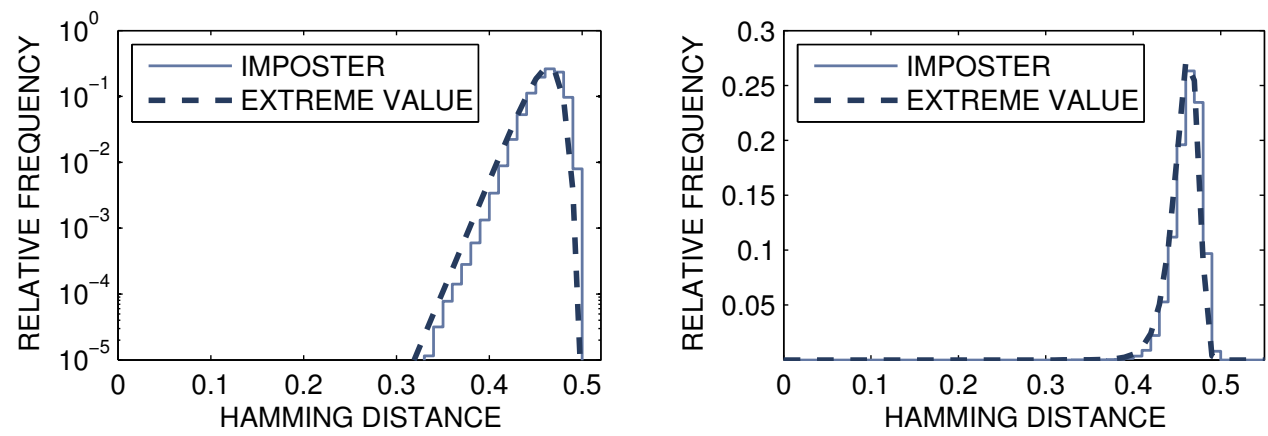

Figure 2.20: The left panel shows the log scale Gumbel extreme value distribution function that provided the best fit to the distribution of imposter HDs generated from synthetic data. The right panel compares Gumbel extreme value distribution function and the histogram distribution of HDs obtained from synthetic data. The temples used to perform experiments are of size 20-by-240.

the Statistics Toolbox in MATLAB and using them in the equation (2.14). For 2,872,800 imposter HDs of synthetic data, the estimated parameters $\mu$ and $\sigma$ are equal to 0.4643 and 0.0129, respectively. The left panel in Fig. 2.20 shows the plot of the Gumbel extreme value distribution with ML estimates in it for the case of synthetic data. Its y-axis is plotted on log scale. The right panel in Fig. 2.20 shows the same plot but in linear scale.

To evaluate the thresholds that result in $1 \% F A R_{1: M}$ for identification performance, the inverse of the function in the expression (2.14) for corresponding $F A R_{1: 1}$ were used. The extrapolation results are displayed in Table 2.5, which is organized as same as Table 2.4 such that the data in Tables 2.4 and 2.5 can be easily compared.For CASIA database, the Gumbel extreme value distribution that provided the best fit to the empirical imposter HDs is described by $\mu=0.4641$ and $\sigma=0.0113$, while for synthetic iris with the same sample size, the distribution is described by $\mu=0.4645$ and $\sigma=0.0128$.

\subsection{Conclusion}

This chapter proposed an anatomy based method for synthesizing iris images with the major purpose to provide the academia and industry with a large database of generated irises to test newly designed iris recognition algorithms. Since synthetic data are known to introduce a bias that is impossible to predict, the data have to be used with caution. We 
Table 2.5: HD threshold and its effect on FARs. The results are obtained using Gumbel extreme value distribution (with 208008 imposter comparisons)

\begin{tabular}{|c|c|c|c|c|}
\hline Database & Estimated & Estimated & \multicolumn{2}{|c|}{ HD Threshold $\gamma$} \\
\cline { 4 - 5 } Size $(M)$ & $F A R_{1: M}$ & $F A R_{1: 1}$ & CASIA & Synthetic \\
\hline $10^{3}$ & $1.0 \%$ & $1.0050 \times 10^{-5}$ & 0.3337 & 0.3169 \\
\hline $10^{4}$ & $1.0 \%$ & $1.0050 \times 10^{-6}$ & 0.3077 & 0.2873 \\
\hline $10^{5}$ & $1.0 \%$ & $1.0050 \times 10^{-7}$ & 0.2816 & 0.2577 \\
\hline $10^{6}$ & $1.0 \%$ & $1.0050 \times 10^{-8}$ & 0.2555 & 0.2282 \\
\hline $10^{7}$ & $1.0 \%$ & $1.0050 \times 10^{-9}$ & 0.2294 & 0.1986 \\
\hline $10^{8}$ & $1.0 \%$ & $1.0050 \times 10^{-10}$ & 0.2033 & 0.1690 \\
\hline
\end{tabular}

believe, however, that the generated data provide an option to compare efficiency, limitations, and capabilities of newly designed iris recognition algorithms through their testing on a large scale data set of generated irises.

The extensive performance comparison of iris images from real and synthetic data sets were performed. The comparison was quantified at three different levels: (i) global layout, (ii) features of fine iris textures, and (iii) recognition performance including performance extrapolation capabilities. 


\section{Chapter 3}

\section{Multi-spectral Iris}

This chapter is devoted to a different problem in the area of iris recognition. Different reflectivity properties of the iris tissue under different spectral illumination make the direct encoding and matching of a color image vs. a NIR image of an iris challenging. In this chapter, an adaptive method to predict NIR channel image from color iris images is introduced. Both visual inspection of the predicted image and the verification performance indicate that the adaptive mapping linking NIR image and color image is a potential solution to the problem of matching NIR images vs. color images in practice. When matched against NIR enrolled image the predicted NIR image achieves significantly high performance compared to the case when the same NIR image is matched against $R$ channel alone.

\subsection{Introduction}

Iris images captured in near infrared (NIR) band are traditional input to iris recognition system [4]. In recent years, however, biometricians turned their attention to iris images acquired in the visible band of electromagnetic spectrum [48]. This trend is supported by a variety of factors: (1) optical cameras in visible range are cheap and characterized by a very high resolution; (2) in terms of applications, most of security and surveillance camera systems (installed for monitoring various human activities) are visible range cameras. Apart from monitoring activities, these cameras may capture face or iris images, which further may be used to authenticate a suspicious or violent individual. Thus iris images acquired by the 
cameras in visible spectrum are query images that have to be compared against enrolled database of irises, which is traditionally formed from the iris images in the NIR range.

Although some publications are available on the topic (for example, publications involving UBIRIS dataset and West Virginia University (WVU) multi-spectral iris dataset), the investigation of matching NIR and visible range iris images has been very limited; and in general the problem of cross spectral matching remains unsolved.

Extracting object reflectivity information across multiple spectral bands is a scope of multi-spectral and hyper-spectral imaging [49,50]. For example, both the surface of the earth (a very general object category) [51] and human face or fingertip (a very specific object category) have different reflectivity responses in different spectral bands [52-54]. This spectral information (often called spectral signatures) can be used to solve many inference problems. It can be used to perform precise segmentation of ground regions with different reflectivity responses, to detect spectral anomalies, or perform classification based on object reflectivity function.

Similar to the application of multi-spectral imaging in geoscience, in biometrics we can use the spectral information to enhance cross spectral biometric matching. Spectral signatures of different regions of the iris, face or fingertip can be estimated from multi-spectral data. This estimate can further be used to predict the appearance of a given spectral representation of a biometric modality in a different spectral band. That is, the estimated relationship is used to map one spectral representation into another spectral representation.

To be more specific, a nonlinear adaptive model to predict the value of the NIR channel from a visible range iris image is proposed. The predicted value of the NIR channel is compared with real NIR iris images by using the classical log-Gabor filter-based algorithm [45]. The results of the performance evaluation are promising. The proposed approach outperforms NIR vs. R cross match comparison.

Recently, Burge and Monaco presented a model for approximating a NIR iris image using features derived from the color and structure of a color iris image in $[55,56]$. In their approach, first, the cluster analysis in the $L^{*} a^{*} b^{*}$ color space on the visible light channels of the multispectral iris images was employed to identify a set of representative color clusters. Next, for each of the clusters, a set of tuples $\left(L^{*}, a^{*}, b^{*}, \mathrm{NIR}\right)$ was constructed. This set 
is used to establish a relationship between the $L^{*} a^{*} b^{*}$ values and NIR values of registered images. The tuples are then augmented with local structural information derived from $k$ multiscale log-Gabor filters $G(w)$, where $w$ is the center frequency of filters. For each cluster, a functional mapping relating $\left(G\left(w_{1}\right), G\left(w_{2}\right), \ldots, G\left(w_{k}\right), L^{*}, a^{*}, b^{*}\right)$ and NIR is constructed using a supervised learning algorithm, which outputs an approximation to the NIR term using the the first $k+3$ terms as inputs.

In our approach, we use RGB color space (the traditional color space) as input to a nonlinear multivariate adaptive mapping and NIR image as output. Furthermore, the camera used for our experiments records three channels: R component, combined $\mathrm{G} / \mathrm{B}$ component and NIR component. Apart from this, Burge and Monaco have not demonstrated any numerical results in their publications.

The remainder of the chapter is organized as follows. Sec. 3.2 describes our proposed methodology. Sec. 3.3 presents numerical results. Conclusions and future work are discussed in Sec. 3.4.

\subsection{Methodology}

Since our methodology for performing iris cross spectral matching requires that images from different spectral bands be well registered, we will first introduce an existing database of multispectral iris which satisfies this assumption. Then we will present the proposed processing and matching methods.

\subsubsection{Multi-spectral Iris Dataset and Data used in Simulations}

Multi-spectral iris data collected at WVU (see [57] for detailed description of the dataset) are used to demonstrate the performance of the proposed method. MS3100 camera manufactured by Geospatial Systems, Inc. was employed for data acquisition. The MS3100 camera is a 3-chip Multispectral digital camera with $1.4 \mathrm{M}+$ pixels per sensor. The multispectral iris dataset collected at WVU involves data of 35 users (68 classes, 232 images). Each iris snapshot outputs data acquired by three different spectral channels: NIR, R and G/B. They are perfectly registered and synchronized and can be used to form a false-color image if those 
three channels are treated as traditional R, G, and B channels. A sample image with falsely mapped NIR into red, red into green and G/B into blue is provided in Fig. 3.1.

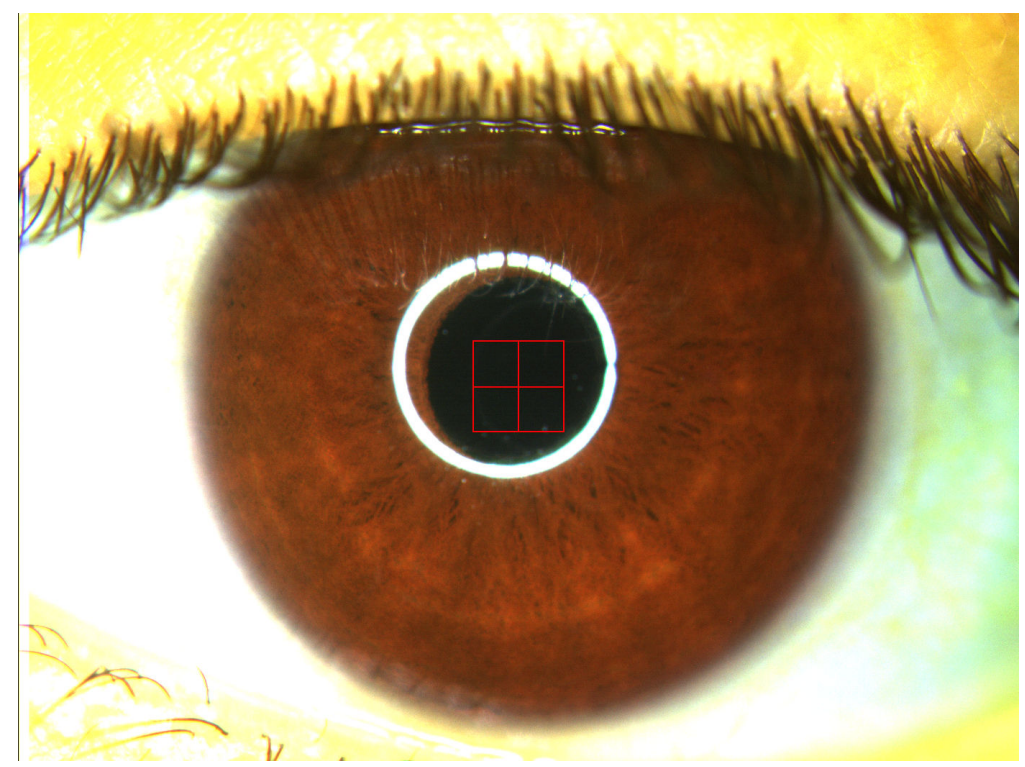

Figure 3.1: An illustration of false color image.

\subsubsection{Proposed Predictive Model}

The variations in human eye color are attributed to varying ratios of eumelanin produced by melanocytes in the iris. Based on the iris color, all irises can be placed in three broad groups: blue, brown, and green. Each group requires different predictive model. Thus, the models for the blue irises, brown irises and green irises have to be designed and trained separately. It has been empirically confirmed that a prediction model trained on a blue iris performs really well only on blue irises. To ensure that the models are equally well trained, sufficient amount of data for training and testing of each model has to be collected.

The predictive model is trained as follows. The problem of training predictive model can be stated as a nonlinear multivariate regression problem, where the predictive model establishes a relationship between input parameters and output parameters. As shown in Fig. 3.2, for each pixel in a single iris snapshot (composed of three channels R, G/B, and NIR) selected for training, the pair of parameters (value of $R$ channel, value of $G / B$ channel) 
form an input and a value of NIR channel form an output parameter. In our simulations described in later sections the predictive model is estimated using a neural network (NN). The type of $\mathrm{NN}$ and the number of hidden layers can be selected based on the amount and type of data involved. Once the mapping is estimated, it is used to predict the output parameter (NIR image) from the input parameters ( $R$ and G/B channels of a query image).

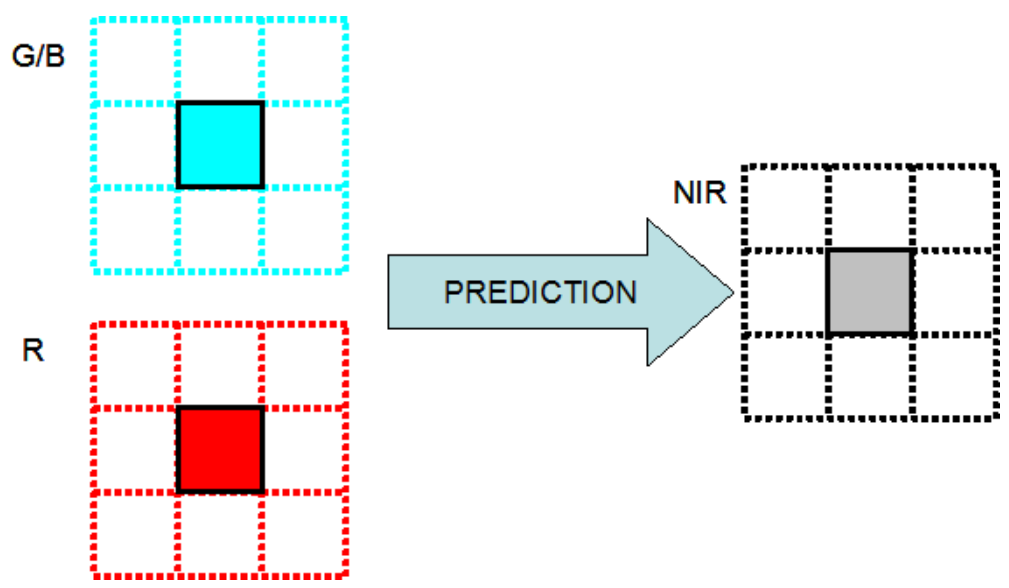

Figure 3.2: Pixel-based prediction.

To ensure reliable model for predictive mapping and robust matching, in place of using only two intensity value as the input parameter (as it is illustrated in Fig. 3.2) we also involve intensity values of the nearest 8-neighborhood. The new prediction strategy is illustrated in Fig. 3.3.

\subsubsection{Neural Network}

Here the nonlinear mapping is estimated using Feed Forward Neural Network (FFNN). The optimal selection of the number of hidden layers (here we selected two) was determined by trading off the complexity of the network versus its predictive performance. For the experiments cross matching multispectral iris images, the first hidden layer of the FFNN is composed of 16 neurons while the second layer is composed of 3 neurons. The training data are divided randomly in two subsets: a learning subset composed of $60 \%$ of training data and a validation subset made of remaining $40 \%$ of data. The training process stops when the mean square error drops below $10^{-5}$. The experimental results described below are obtained 


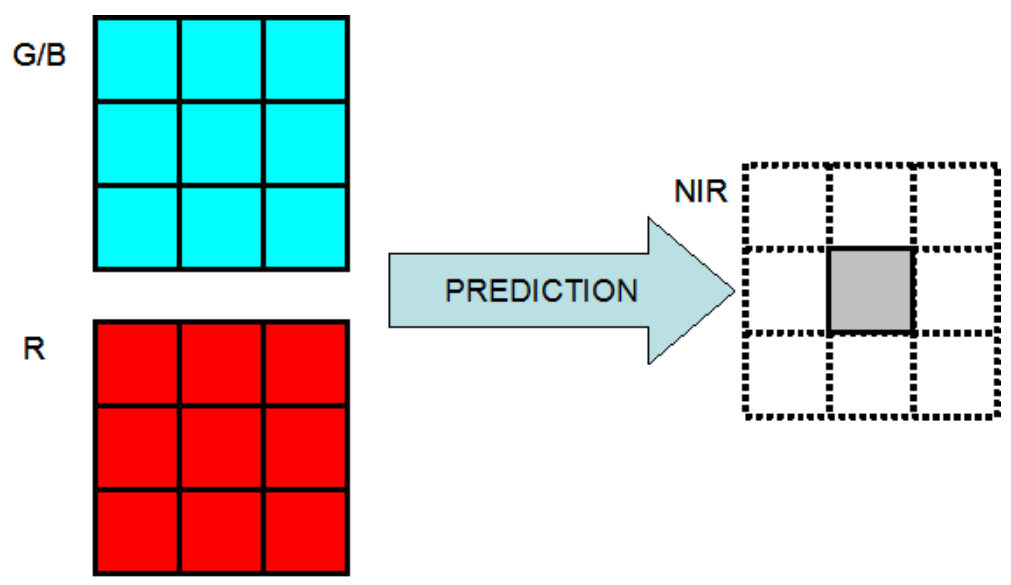

Figure 3.3: Neighborhood-based prediction.

using functions from Neural Network Toolbox in $M A T L A B^{T M}$.

\subsubsection{Training}

Each training image is first segmented to guarantee that only iris region is selected for training the proposed predictive model.

As shown in block-diagram in Fig. 3.4, training the predictive model requires a vector of 18 input parameters and a single output parameter. In our implementation, first 9 input parameters come from the $\mathrm{G} / \mathrm{B}$ channel and the following 9 input parameter come from the $\mathrm{R}$ channel.

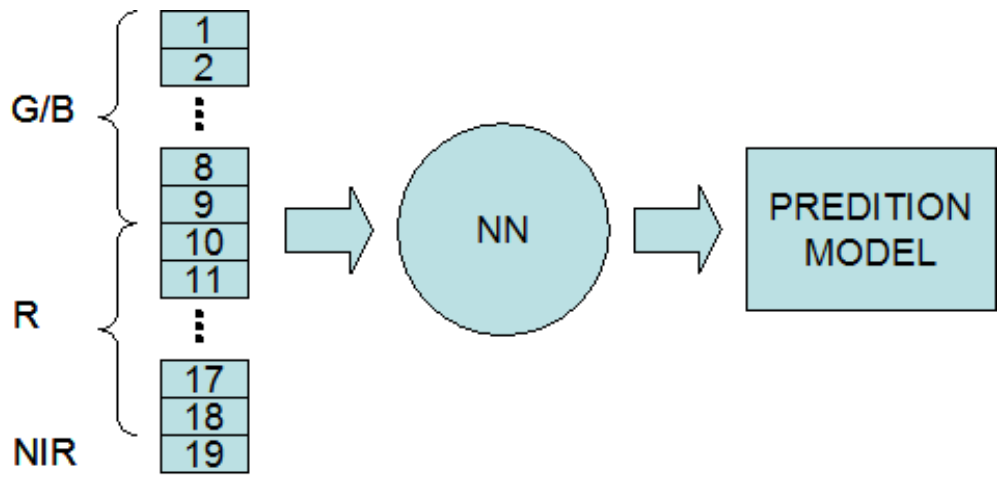

Figure 3.4: Block-diagram of the training procedure.

Since estimation of predictive model depends on initial condition, we run the training 
experiment a number of times and select the model, which produces the minimum genuine score. This score is formed by comparing predicted NIR data versus the true NIR data.

\subsubsection{Testing}

During testing the estimated mapping is used to predict NIR channel from the inputs ( $\mathrm{R}$ channel,G/B channel). The testing procedure is described in Fig. 3.5.

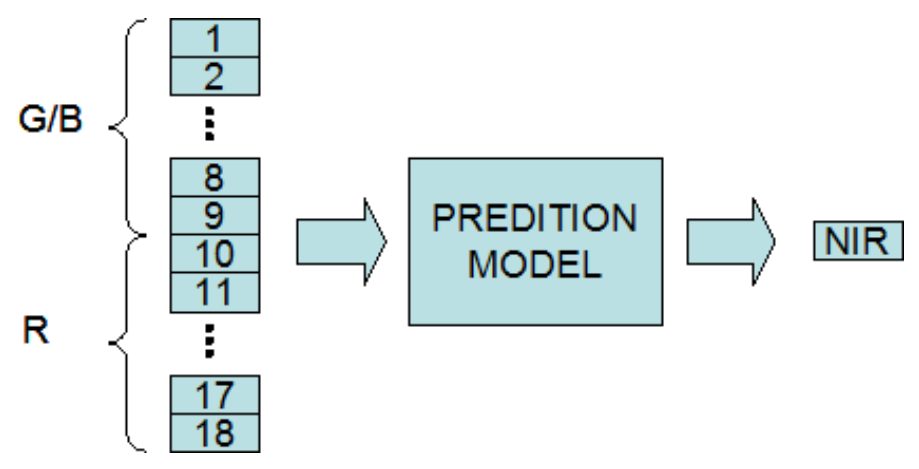

Figure 3.5: The block-diagram of the testing procedure.

\subsubsection{Model Selection Criterion}

Since different prediction models produce different results, the question of how to choose the best model was raised. There is a variety of theoretical and practical model selection criteria. In this work we used only data available to us, that is, information in $\mathrm{G} / \mathrm{B}$ and $\mathrm{R}$ channels. To select the best model the median intensity values of $\mathrm{G} / \mathrm{B}$ and $\mathrm{R}$ channels can be used. The medians are evaluated based on the entire available area within the images. The optimal predictive model $I$ is selected if it minimizes the following cost function:

$$
\left(\left(\text { Test }_{G / B}-\text { Train }_{G / B}^{i}\right)^{2}+\left(\text { Test }_{R}-\text { Train }_{R}^{i}\right)^{2}\right),
$$

where Test $_{G / B}$ and Test $_{R}$ are the median values of $\mathrm{G} / \mathrm{B}$ and $\mathrm{R}$ channels for the testing image

and $\operatorname{Train}_{G / B}^{i}$ and $\operatorname{Train}_{R}^{i}$ are the median values of $\mathrm{G} / \mathrm{B}$ and $\mathrm{R}$ channels for the $i$ th training image.

An alternative solution would be to involve several prediction models (in place of one). These models are then used separately to predict the output. The best predictive model is 
the one, which optimizes the verification performance. For example, in our implementation, for each testing image, 10 different prediction results were generated by 10 different predictive models. When a query visible light image is compared with NIR enrolled images, 10 different matching scores (Hamming Distances) for each NIR enrollment are recorded. The minimum score is selected as the best score. This approach is especially useful when dealing with a set of images of the same iris acquired under different environmental conditions.

\subsubsection{Illustration of Predicted Results}

An example showing an iris snapshot (its original 3 channels: R, G/B, and NIR) and a predicted NIR channel are shown in Fig. 3.6. From this example, it can be easily seen that the predicted NIR channel visually is very similar to the original NIR channel and substantially differs from the R and G/B channels. Note, however, due to the predictive model, which involves neighbors, that the predicted NIR channel is slightly over-smoothed compared to the original NIR channel.

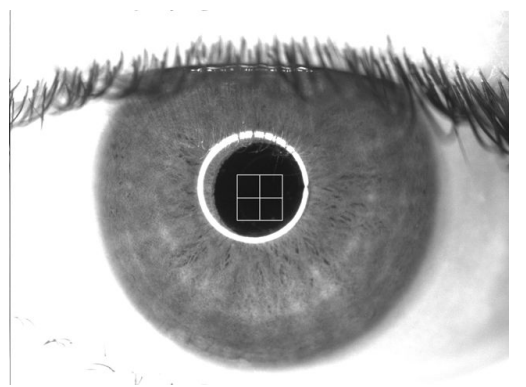

(a)

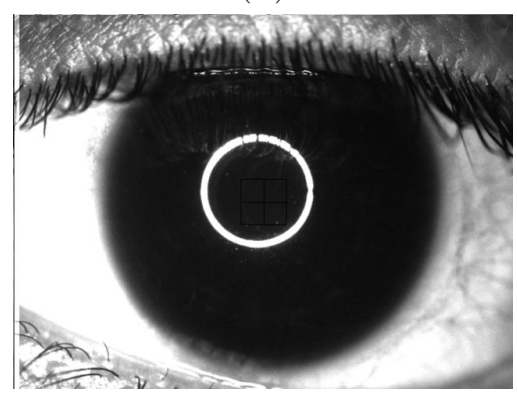

(c)

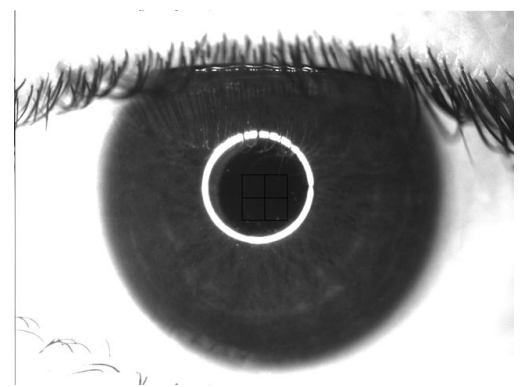

(b)

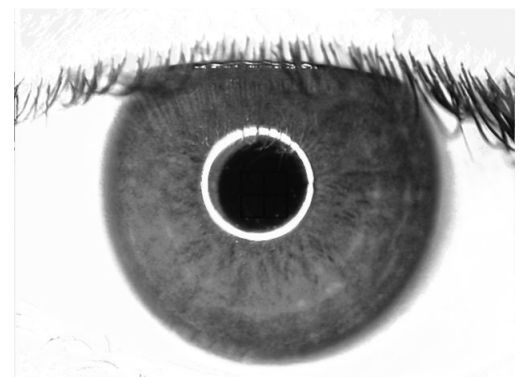

(d)

Figure 3.6: (a) The original NIR channel; (b) the R channel; (c) the G/B channel; and (d) the predicted NIR channel using (b) and (c) as the input to the predictive mapping function. 
For a close examination, the unwrapped iris patterns are displayed in Fig. 3.7 and 3.8.

(a)

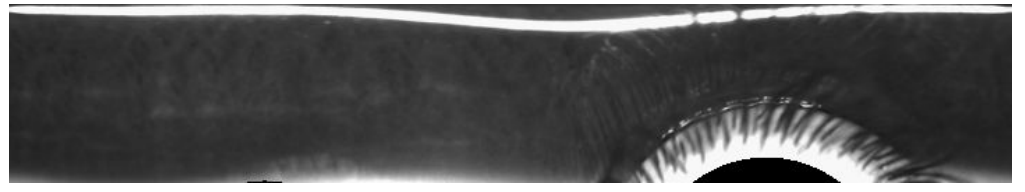

(b)

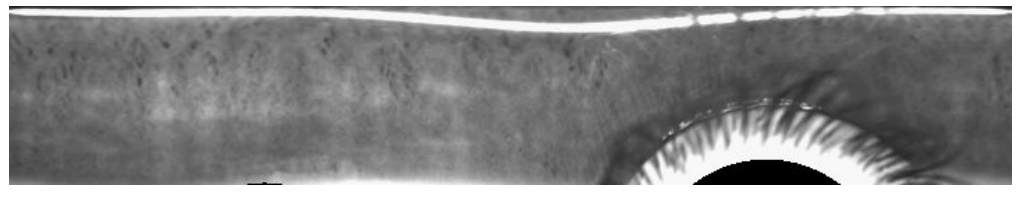

(c)

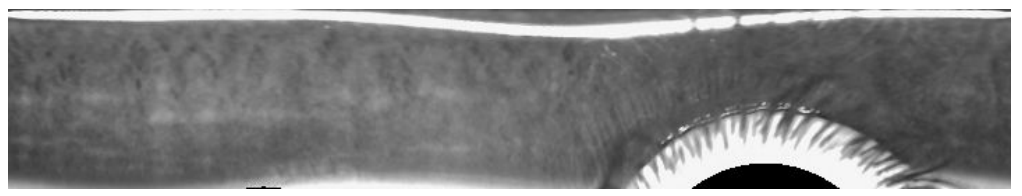

Figure 3.7: Unwrapped iris templates: (a) R channel; (b) NIR channel; and (c) the predicted NIR channel.

(a)

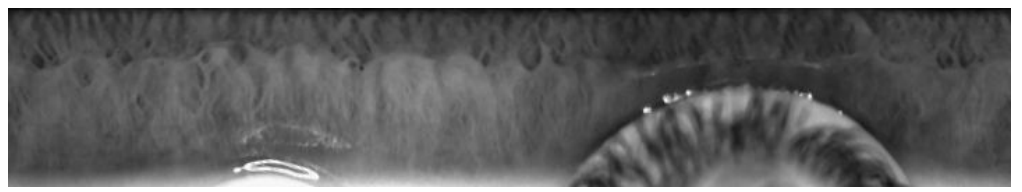

(b)

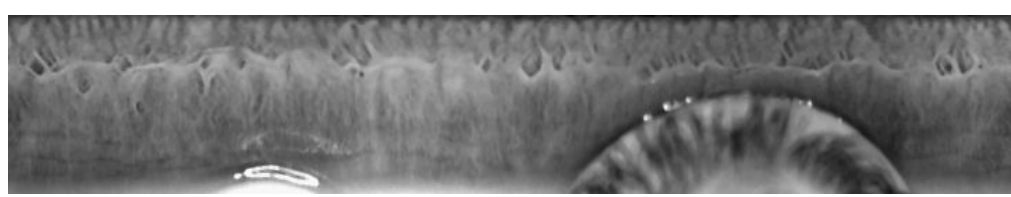

(c)

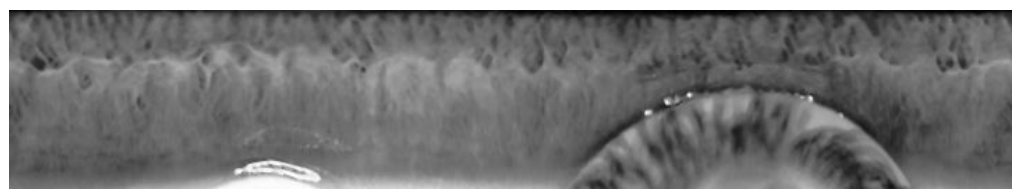

Figure 3.8: Unwrapped iris templates: (a) R channel; (b) NIR channel; and (c) the predicted NIR channel.

\subsection{Numerical Results}

To evaluate recognition performance of predicted NIR iris images, all iris images from the testing set were segmented using the robust iris segmentation algorithm developed by 
Zuo and Schmid [58]. The segmentation was applied to NIR channel data only. Then the extracted segmentation parameters were used to segment the other spectral channels.

After segmentation, the data were encoded using an enhanced version of the Masek's iris encoding algorithm (the details are provided in [59]). Three similarity matrices were formed: (1) the first matrix compared the true NIR imagery versus the true NIR imagery. This case is treated as the best achieved performance; (2) the second matrix compares the true NIR images versus predicted NIR data; and (3) the third matrix compares the true NIR versus the $\mathrm{R}$ channel. Note that $\mathrm{G}$ and $\mathrm{B}$ channels were not involved in performance evaluation. Compared to $\mathrm{G}$ and $\mathrm{B}$ channels, $\mathrm{R}$ iris image strongly resembles NIR iris image. When involving simple fusion rules at the match score level, the matching performance of $\mathrm{R}$ channel is much higher compared to the matching performance due to $\mathrm{G}$ and $\mathrm{B}$ channels. Therefore, the match score of $\mathrm{R}$ channel strongly dominates the decision making process. Here the comparison of NIR channel vs. $\mathrm{R}$ channel is treated as the baseline comparison. During numerical evaluations, 5 out of 150 testing images were discarded due to poor segmentation. The remaining data were used to plot receiver operating characteristic (ROC) curves.

The results of performance comparison are summarized in Fig. 3.9 and in Table 3.1. Fig. 3.9 shows the ROC curves for the three comparisons above. The ROCs are displayed as a plot of Genuine Acceptance Rate (GAR) versus False Acceptance Rate (FAR). Note the performance improvement due to involvement of the predictive model compared to the direct cross spectral matching of NIR data against R-channel data. The corresponding values of the average genuine scores, the average impostor scores, d-prime $\left(d^{\prime}\right)$ and Equal Error Rate (EER) are summarized in Table 3.1. It shows the improved average performance of the predicted NIR data compared to performance of R-channel.

Table 3.1: The results of three cross comparisons

\begin{tabular}{|c|c|c|c|c|}
\hline & $\begin{array}{c}\text { Mean of } \\
\text { Genuine Scores }\end{array}$ & $\begin{array}{c}\text { Mean of } \\
\text { Impostor Scores }\end{array}$ & $d^{\prime}$ & EER \\
\hline NIR vs. NIR & 0.0505 & 0.3986 & 10.3211 & 0 \\
\hline NIR vs. P_NIR & 0.0841 & 0.3825 & 8.7493 & 0.00034 \\
\hline NIR vs. R & 0.1177 & 0.4012 & 6.3913 & 0.00051 \\
\hline
\end{tabular}

Results in Table 3.1 indicate that the improved performance is due to the reduction of 


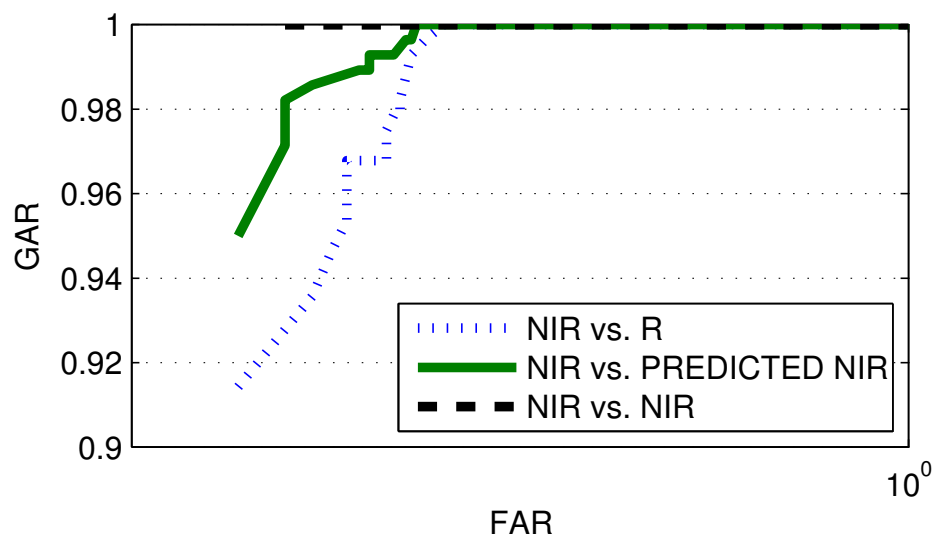

Figure 3.9: Performance improvement due to the proposed predictive model.

values of genuine scores. When comparing two cases (Predicted NIR, NIR) genuine comparison and (R, NIR) genuine comparison, in 267 out of 280 cases (Predicted NIR, NIR) comparison outperforms (R, NIR) comparison. Similarly, when comparing two cases (NIR, NIR) genuine comparison and (Predicted NIR, NIR) genuine comparison, in 271 out of 280 cases (NIR, NIR) comparison outperforms (Predicted NIR, NIR) comparison. However, few outliers are observed in both cases. We introduce gain (loss) of the performance for these genuine comparisons and define it as the difference between the matching scores of one probe image and the matching score of another probe image compared to the same NIR target image. The cumulative gain (loss) of performance can be visualized through plotting histograms of performance gains on genuine comparisons (see Fig. 3.10).

The prediction also was proven to be robust with respect to imperfect segmentation. We conducted the following experiment on all 150 images. All segmentations of $\mathrm{R}$ channel and predicted NIR channel were slightly distorted in radial direction. The segmentation of NIR iris images was not modified. The ROC curves for these experiments are displayed in Fig. 3.11. Few statistics for the same case are summarized in Table 3.2. Overall, the performance dropped slightly, but the relative position of the ROC curve of predicted NIR versus the true NIR remained unchanged. 
Table 3.2: The results of three cross comparisons with imperfect relative segmentation of $\mathrm{R}$ and Predicted NIR channels

\begin{tabular}{|c|c|c|c|c|}
\hline & $\begin{array}{c}\text { Mean of } \\
\text { Genuine Scores }\end{array}$ & $\begin{array}{c}\text { Mean of } \\
\text { Impostor Scores }\end{array}$ & $d^{\prime}$ & EER \\
\hline NIR vs. NIR & 0.0671 & 0.4093 & 7.9266 & 0.0069 \\
\hline NIR vs. P_NIR & 0.0967 & 0.3907 & 6.8603 & 0.0131 \\
\hline NIR vs. R & 0.1300 & 0.4118 & 5.5570 & 0.0137 \\
\hline
\end{tabular}

\subsection{Conclusions and Future Work}

We have demonstrated that a well designed predictive mapping, which is used to map color image into Predicted NIR image, is a promising approach to improve cross spectral iris recognition. Application of a well designed predictive mapping, as shown here, resulted in $10 \%$ improvement when color image is mapped into NIR image first and then compared against a NIR image. This improvement is measured with respect to $R$ channel versus NIR image comparison (which is treated as the worst possible comparison).

Similar experiment have been performed on multispectral face images. The results, unfortunately, are not as promising as the results for iris biometrics. Designing a new predictive mapping to improve performance of cross spectral face recognition is ongoing work in our lab. 
(a)

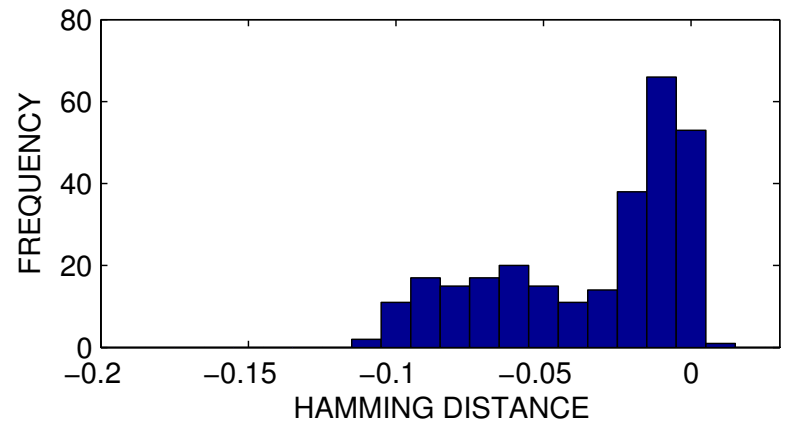

(b)

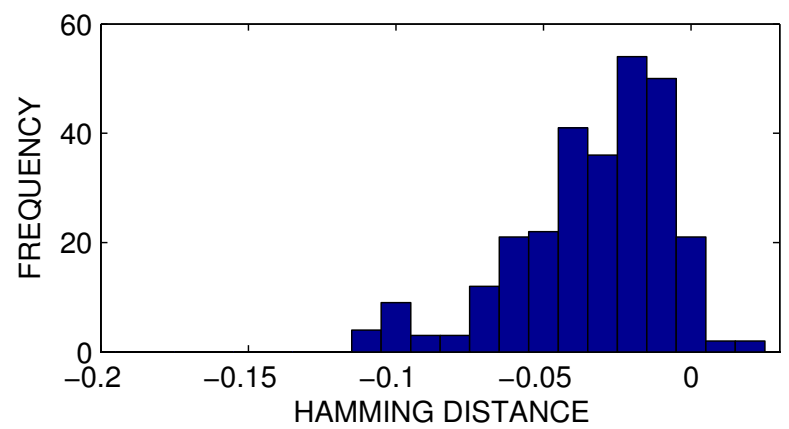

(c)

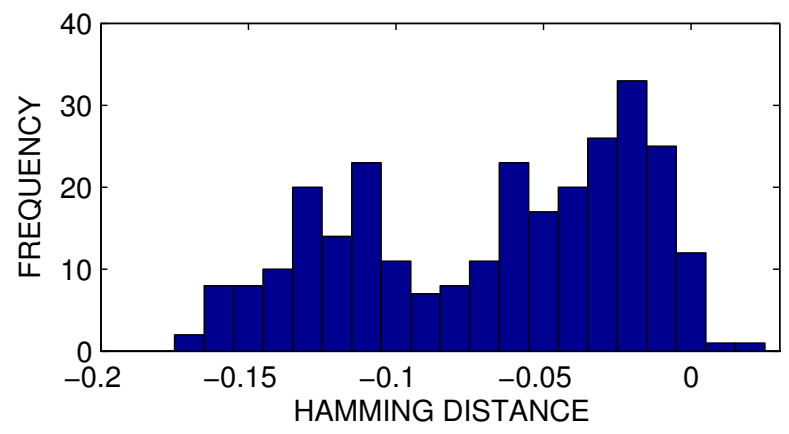

Figure 3.10: Performance gains on genuine scores: (a) (Predicted NIR, NIR) vs. (R, NIR); (b) (NIR, NIR) vs. (Predicted NIR, NIR); and (c) (NIR, NIR) vs. (R, NIR). 


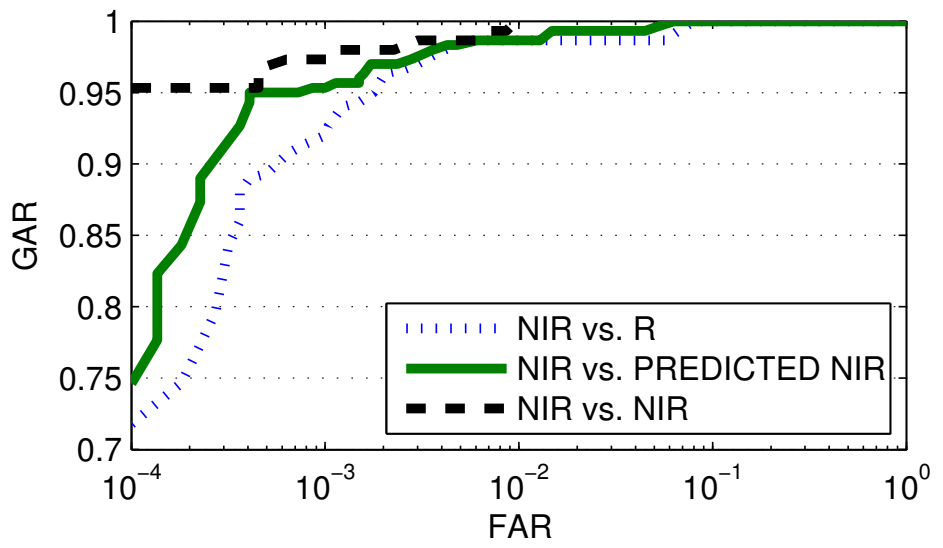

Figure 3.11: The performance improvement due to the proposed predictive model when different segmentations for $\mathrm{R}$ and predicted NIR channels were used. 


\section{Chapter 4}

\section{Iris Quality}

This chapter focuses on measures of iris sample quality for iris video. In the field of iris recognition, evaluation of quality of images has a number of important applications. These include image acquisition, enhancement, and data fusion. Iris image quality metrics designed for these applications are used as figures of merit to quantify degradations or improvements in iris images due to various image processing operations.

\subsection{Introduction}

Iris image quality assessment is an important research thrust recently identified in the field of iris biometrics [60], [61], [62]. This research is tightly related to the research on nonideal iris. Its major role is to determine, at the stage of data acquisition or at the early stage of processing, what amount of information for the purpose of processing, recognition, and fusion an image contains. Is it useful enough for performing further processing steps or should be discarded? Is it informative enough for being combined with other images and result in improved recognition performance? The quality metrics play an important role in automated biometric systems for three reason: (1) system performance (segmentation and recognition), (2) interoperability, and (3) data enhancement.

Image quality assessment plays an important role in automated biometric systems. Low quality images may have poor lighting, defocus blur, off-angle, and heavy occlusion, which have a negative impact on even the best available segmentation algorithms. Even with perfect 
segmentation, information losses due to distortions of iris texture or iris image intensity may cause serious problems for encoding and matching algorithms. At the same time, an image of good quality (as it is predicted by an image quality measure or based on visual evaluation) may not be a good iris biometric sample, as it may result in a low matching score when the encoded iris image is compared against an enrolled iris sample from the same iris class. Therefore, iris biometric quality should not be limited to iris image quality.

Previous work on iris image quality can be placed into two categories: local and global analyses. Zhu et al. [63] evaluate quality by analyzing the coefficients of particular areas of iris's texture by employing discrete wavelet decomposition. Chen et al. [64] classify iris quality by measuring the energy of concentric iris bands obtained from 2-D wavelets. Ma et al. [65] analyze the Fourier spectra of local iris regions to characterize out-of-focus and motion blur and occlusions. Zhang and Salganicaff [66] examine the sharpness of the region between the pupil and the iris. Daugman [8] and Kang and Park [67] characterize quality by quantifying the energy of high spatial frequencies over the entire image region. Belcher and $\mathrm{Du}[68]$ propose a clarity measure by comparing the sharpness loss within various iris image regions against the blurred version of the same regions. The major feature of these approaches is that the evaluation of iris image quality is reduced to the estimation of a single $[8,64,66,67]$ or a pair of factors [65], such as out-of-focus blur, motion blur, and occlusion.

Iris quality should not be limited to one or two quality factors. All factors that will affect recognition performance should be counted as iris quality factors. A broader range of physical phenomena that can be observed in nonideal iris imagery was characterized by Kalka et al. [69], [70]. The proposed factors include out-of-focus and motion blur, occlusion, specular reflection, illumination, off-angle, and pixel count. The strength of the phenomena and its influence was evaluated through modified or newly designed iris quality metrics. Based on the extensive analysis carried out by the authors of [69] and [70], these factors affect the segmentation and ultimately recognition performance of iris recognition systems.

This chapter elaborates on the factors in [70] and introduces new factors that can be used to evaluate near-infrared (NIR) video and image quality. The main contributions of this chapter are as follows. (1) A fast global quality evaluation procedure for selecting the 
best frames from a video or an image sequence is introduced. (2) A number of new local quality measures for the iris biometrics are introduced. The performance of these quality measures is carefully evaluated. Since performance of iris recognition systems is evaluated in terms of the distributions of matching scores and recognition probability of error, from a good iris image quality metric it is also expected that its performance is linked to the recognition performance of the biometric recognition system.

\subsection{Frame Selection}

Modern iris capture devices are often equipped with an algorithm performing selection of high quality frames. Each frame can be further segmented, and the final quality score can be used to select the frame. However, complex segmentation procedure can not follow the frame rate. Then a simple quality factor will be preferred for this purpose. For instance, Daugman suggested using the focus measure to select the best iris frames [8]. For a single value quality score, local maximum on the time axis can be used to select the best quality frames from a NIR iris video.

However, for images captured at a distance, it will not be good to use the whole image to evaluate the quality factor because of the short DOF (Depth of Field). The iris area is just a small part of the frame and there may be two irises exist in a same image. The iris detection and the quality analysis will be done at the same time.

\subsubsection{MBGC Data}

Building on the challenge problem and evaluation paradigm of ICE (Iris Challenge Evaluation) 2005 and ICE 2006 [71], the Multiple Biometric Grand Challenge (MBGC) for the first time introduced middle distance near-infrared (NIR) face video (may have one or both irises in each frame) [72]. A system which is similar to the system described in [6] was used for the data collection. On a good quality frame, 8 clear specular reflections can be found close to the center of the pupil. This feature can be used to detect the iris area and measure the level of focus.

For a NIR iris video, first the iris region is detected and cropped. Then the best quality 
cropped area is selected by using the focus level. To deal with two irises in the same frame, a quality map is used to find local quality maxima which may be attributed to left and right irises or to a falsely detected iris.

\section{Iris Detection}

The iris detection is based on the assumption that a strong specular reflection exists inside of the iris area (including pupil). Typically, the intensity value of the specular reflection is very high compared to the intensity value of the iris area. Therefore, the gradient information can be used to locate the eyes within a frame. This is accomplished as follows.

1. Two 2D-order statistic filter, one returning the maximum intensity value and the other returning the minimum intensity value over a neighborhood of size $3 \times 3$, are applied locally. The difference of the intensity values within each $3 \times 3$ neighborhood are compared to a threshold, denote it as $\gamma_{1}$.

2. A threshold $\gamma_{2}$ corresponding to the value of iris intensity is evaluated based on the histogram of the considered frame.

3. The areas of the frame simultaneously satisfying conditions in (1) and (2) are selected as the potential candidates for being the iris area.

4. Since the previous steps may generate a large number of candidates, an additional geometric information is involved to rule out false iris regions. We use the minimum distance between the eyes as a means to deal with false iris regions.

An iris detection example is provided in Fig. 4.1.

\section{Quality Map}

Once the Iris Detector returns the coordinates of the iris location in a frame, a quick evaluation of iris image quality is performed. Following the Daugman's procedure for selecting the best iris frames [8], we evaluate the level of blur in detected iris region. Processing and encoding every frame in a video is a time-intensive operation. An alternative solution is to select few frames containing high quality iris regions and use them for iris recognition. 


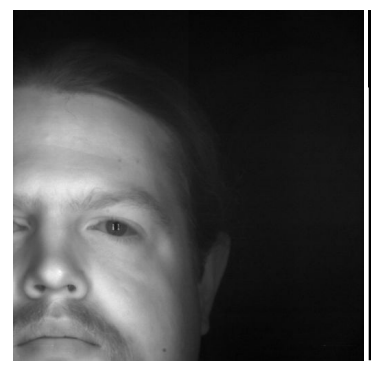

(a)

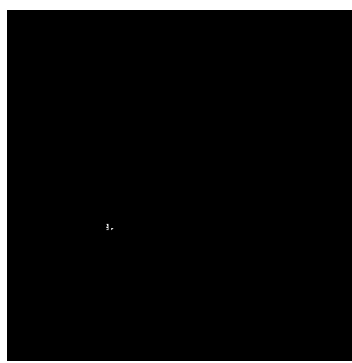

(d)

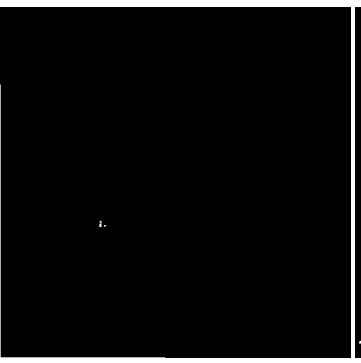

(b)

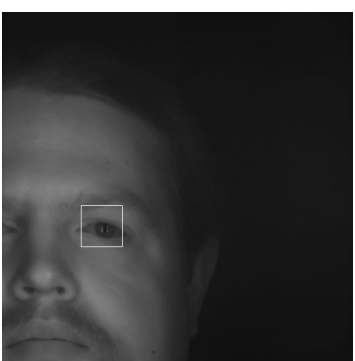

(e)

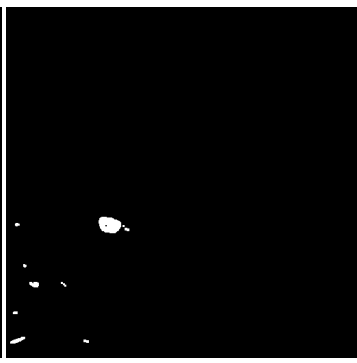

(c)

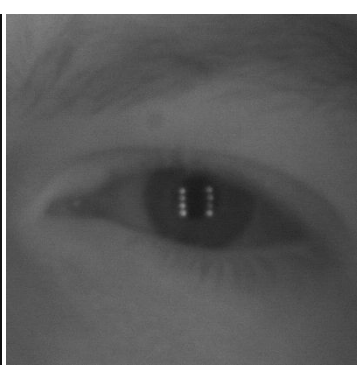

(f)

Figure 4.1: Iris detection for a single frame from the NIR face video: (a) the original frame (from MBGC dataset), (b) clear edges (c) possible iris areas, (d) final candidates, (e) the iris detection result, and (f) the cropped area.

We introduce a global quality map that provides relative information about the quality of the iris regions in different frames.

When each frame is processed, the best quality iris area candidate for each location will be updated. If the current frame have a better candidate than all frames before at that location, then the corresponding quality value and frame number will be updated to the values of the current frame. If the current frame is a worse candidate than the last frame at that location for the first time, then the last frame may be a local maximum in time domain for that location. Corresponding iris area from the last frame will be selected for further examination. After the video is processed, a global quality map with the best quality iris area marked at every location also is finished. At this time, a local maximum of the quality map will be found (these are local maxima in the spatial domain and the global maxima in the time domain), and corresponding iris areas will be selected for further processing. 


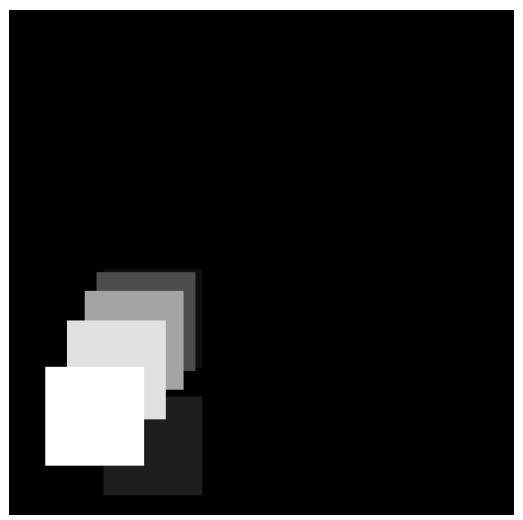

Figure 4.2: Shown is the quality map for the video sequence displayed in part in Fig. 4.1.

\subsection{Quality Evaluation}

After few video frames are selected based on the video quality map, the detected iris regions are segmented and encoded. Simultaneously, local iris quality factors are evaluated. These quality factors can be later used to enhance performance of an iris recognition system alone or of a multimodal system with iris being one of the modalities. In the following subsection a set of new individual iris biometric quality factors are introduced and procedures to evaluate them are described. The factors are segmentation scores, interlacing, illumination, lighting, occlusion, pixel count, dilation, off-angle, and blur.

\subsubsection{Segmentation Scores}

Since the most of local iris quality measures are applied to segmented iris images, the metrics evaluating the precision of the segmentation should be given a higher priority compared to other factors. Two segmentation scores $Q_{p_{-} \text {seg }}$ and $Q_{i_{-s e g}}$ introduced in [73] can be used as two distinct quality metrics related to the segmentation itself. These metrics analyze the gradient values along the pupil and limbic boundaries. Larger value of the measures indicate more precise segmentation. 


\subsubsection{Interlacing}

Poor interlacing is a disturbing artifact. Interestingly, a poorly interlaced image may result in a high focus score in spite of strong defocus of either even or odd lines. These artifacts should be detected. If there is a large difference between odd and even lines then there must be a clear motion related interlacing effect. The image may be either discarded, or divided to two sub-images: odd rows and even rows. The difference between odd rows and even rows Inter ${ }_{1}$ can be calculated as

$$
\text { Inter }_{1}=\frac{\sum_{i=1: 2: m-1}\left(\sum_{j=1: n}\left|I_{(i, j)}-I_{(i+1, j)}\right|\right) * 2}{m * n}
$$

for an image $I$ with $m$ rows and $n$ columns. The function must be normalized by subtracting Inter $_{2}$ calculated using odd or even rows only

$$
\text { Inter }_{2}=\frac{\sum_{i=1: m-2}\left(\sum_{j=1: n}\left|I_{(i, j)}-I_{(i+2, j)}\right|\right)}{(m-2) * n}
$$

resulting in

$$
Q_{\text {interlacing }}=\text { Inter }_{1}-\text { Inter }_{2} .
$$

Note that the high values of $Q_{\text {interlacing }}$ indicate poor interlacing.

\subsubsection{Illumination}

The contrast of the image is mainly determined by the level and strength of the illumination. The illumination level is the mean intensity value of the iris area:

$$
Q_{\text {illumination }}=\frac{\sum_{\text {unaffected iris area }} I_{(i, j)}}{\sum_{\text {unaffected iris area }} 1} .
$$

To get a more precise estimation of this factor, only unaffected (by occlusion or specular reflections) area is considered. The value that $Q_{\text {illumination }}$ can take ranges from 0 to 255 . This factor can be affected by the color of the iris. Large values of the measure indicate high illumination value. 


\subsubsection{Lighting}

Sided or uneven illumination of the iris often results in performance degradation. Illumination pattern can be treated as a low frequency signal that distorts encoded iris images. The variance of the mean intensity evaluated over small blocks is proposed as a measure of the uneven illumination. The calculation of the lighting factor is similar to the procedure described in [70], but without normalization to $[0,1]$. Note that bad lighting condition is characterized by a large value of the metric.

\subsubsection{Occlusion}

This attribute measure how much of the iris is occluded by other objects such as eyelid, eyelashes and specular reflections. The proposed metric evaluates the percentage of the unoccluded area in the final unwrapped template

$$
Q_{\text {ocllusion }}=\frac{\sum_{(i, j):} 1}{\sum_{(i, j)} 1} 1
$$

where $M$ is the binary unwrapped noise mask of the unwrapped iris template where true (1) means information at that location can not be used. The usage of the percentage can reduce the correlation between this quality factor and the resolution factor. This quality factor is similar to the pixel count factor in [70]. Large values of the metric indicate smaller occlusions.

\subsubsection{Pixel Count}

To distinct it from the occlusion factor, pixel count finds the total iris area even it is affected by occlusions, that is,

$$
Q_{\text {pixel_count }}=\sum_{\text {iris area }} 1 .
$$

Large values of the metric correspond to high pixel counts. 


\subsubsection{Dilation}

The dilation factor measures the degree of the pupil dilation. The high the dilation of the pupil, the high the compression of the iris texture and the less information is available for iris recognition. The value of the dilation factor is calculated by taking the ration of $Q_{\text {pixel_count }}$ and the total iris and pupil area

$$
Q_{\text {dilation }}=\frac{\sum_{\text {iris area }} 1}{\sum_{\text {iris area }} 1+\sum_{\text {pupil area }} 1} .
$$

The value $Q_{\text {dilation }}$ takes is between 0 and 1 . This factor also affects pixel count. Note that small pupil dilations are characterized by large values of the metric.

\subsubsection{Off-angle}

This factor measures the relative orientation of the iris with respect to the camera. Assuming that the frontal view iris has a circular shape, the off-angle view becomes an ellipse. The off-angle quality factor is a ratio of the two main axes of the ellipse fitted into the iris boundary. These values are obtained after the iris has been segmented.

$$
Q_{o f f-a n g l e}=\frac{b}{a}
$$

where $b$ is the minor axis and $a$ is the major axis of an ellipse. Note that the large values of the metric indicate that that the image is close to frontal view.

\subsubsection{Blur}

Both motion and defocus blurs are treated simultaneously. The proposed method uses spectral components of an iris image and involves a number of preprocessing steps.

First, the area of the interest is selected based on the segmentation result. After the parameters of the ellipse such as the ellipse center $\left(x_{i}, y_{i}\right)$, the major axis $a$ and the minor axis $b$ fitted into the iris region are obtained, we set $250 \%$ length of the major axis $a$ as the size of the window and select the iris center $\left(x_{i}, y_{i}\right)$ as the window center. 
Then a small median filter is applied to denoise the image. To compensate the resolution difference, every area of the interest is normalized to $151 \times 151$. This size is approximately selected based on the acceptable iris resolution: 120 pixels across the iris. Then a 2D FFT transform is carried to this image in order to extract the frequency information $P$

$$
P=\log _{10}\left|F F T\left(I_{\text {crop }}\right)\right|,
$$

where $I_{\text {crop }}$ is the cropped iris area after the denoising and the normalization.

After the power distribution of $P$ is analyzed, its central area is used to calculate the proper threshold. Currently we select the average power of a centered 13 pixel diamond shaped area (distance to the center of the power spectrum is less than 4) as the threshold $\gamma$

$$
\gamma=\frac{\sum_{\left((i-76)^{2}+(j-76)^{2}\right)<16} P_{(i, j)}}{13 * 1.5} .
$$

Then the number of location with a higher power value than the threshold is counted. If the number of locations is large, then the power distribution is flat. As the pupil area usually contributes a large number of low frequency component, an adjustment part involving the dilation information is added. Then the final expression for the $Q_{b l u r}$ becomes:

$$
Q_{\text {blur }}=\left(\sum_{P_{(i, j)}>\gamma} 1\right) *\left(1+Q_{\text {dilation }}^{6}\right) .
$$

An example illustrating some steps in evaluation of the blur quality score is provided in Fig. 4.3. This image results in the final blur score 5953.6.

Larger values of the metric correspond to a smaller amount of blur.

\subsubsection{Fusion}

The quality factors (metrics) can be used individually or combined into a single score through a simple static or an adaptive rule. Among static rules the simple sum rule is a computationally efficient method. More complex (adaptive) rules such as Bayesian, DempsterShafer, weighted Sum, or any previously designed fusion strategy to combine classifiers can also be used to combine quality metrics into a single score. These rules are more fundamental and flexible, but require intensive computations.

Our current task is to come up with a super-combination scheme. 


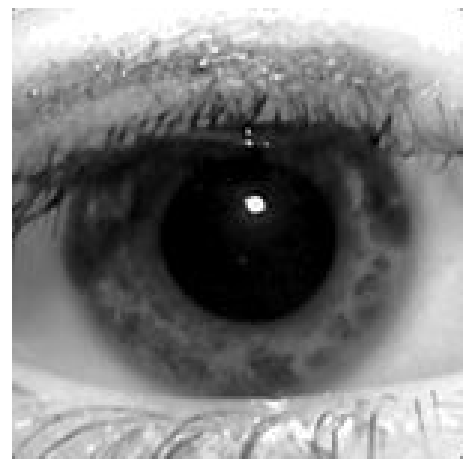

(a)

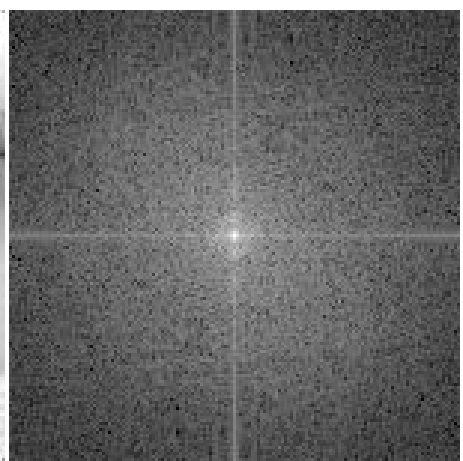

(b)

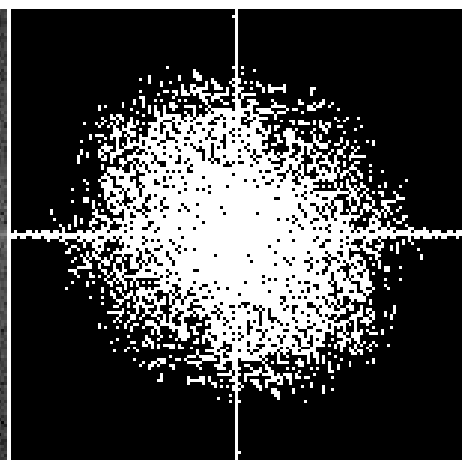

(c)

Figure 4.3: Example of blur estimation (a) the cropped area, (b) the power spectrum and (c) the results of thresholding.

\subsection{Results}

All experiments were performed using ICE 2005 dataset [74]. The enhancement, encoding and matching procedures followed Daugman's implementation. Since performance of any iris recognition system is evaluated in terms of the distributions of matching scores and recognition probability of error, from a good iris quality measure it is also expected that its performance is linked to the recognition performance of the recognition system.

We perform a number of experiments. For each individual factor, the ICE 2005 dataset was used to form three subsets of images. The first subset was composed of the entire ICE dataset. To form the second and the third subsets, we involved the distribution of values of a selected quality factor. The second set included all images with the value of selected quality factor exceeding 0.75 th qauntile. The third set was composed of all images with the value of selected quality factors exceeding 0.9 th quantile.

The panes in Fig. 4.4 and Fig. 4.5 each displays three Receiver Operating Characteristic sets obtained using data in subsets 1,2 , and 3 . Note that all results can be placed into those based on a relative quality score (in our case, it is the difference of two quality values for two distinct images) and those based on an absolute measure. Examples of relative measures include interlacing, illumination, pixel count and off-angle (Fig. 4.5). The other measures were used as absolute.

From Fig. 4.4 and Fig. 4.5 regardless of the type of the measure, the difference between 
ROCs formed from the three subsets of ICE 2005 dataset are quite noticeable. This indicates that each individual factor proposed in this work does influence recognition performance of a Gabor filter-based system.

(a)

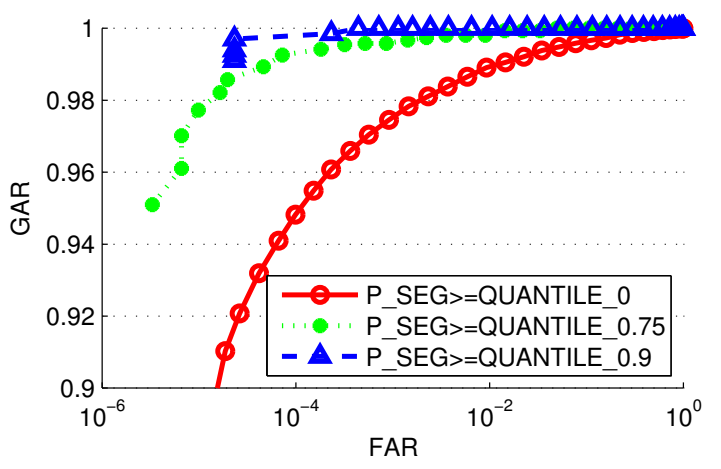

(b)

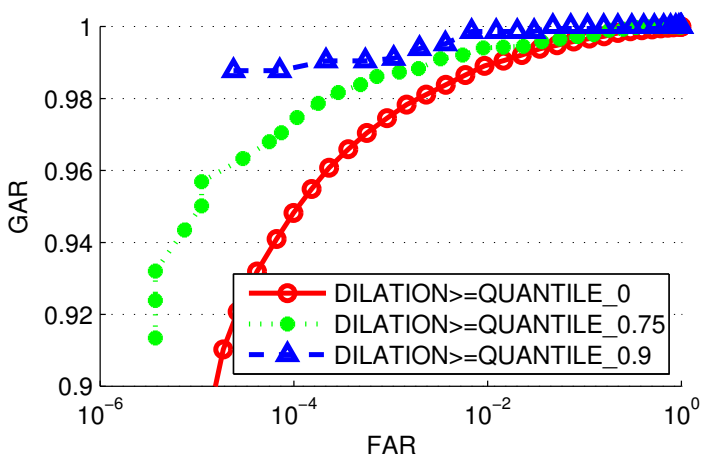

(c)

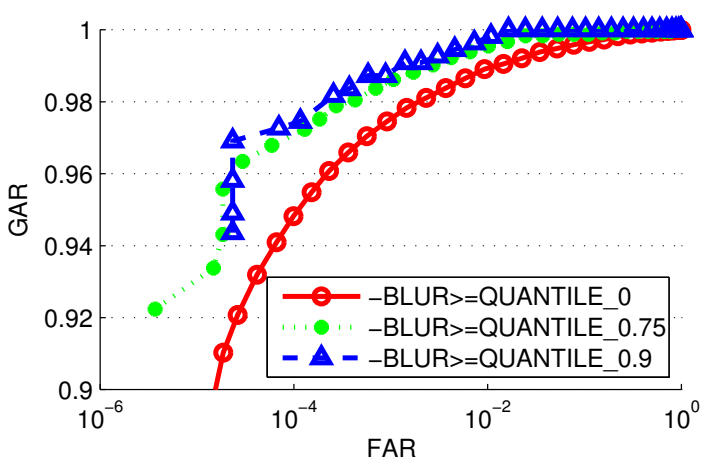

Figure 4.4: ROC curves for ICE2005 dataset (a) selecting images using pupil segmentation score; (b) selecting images using dilation measure; and (c) selecting images using minus blur measure. 


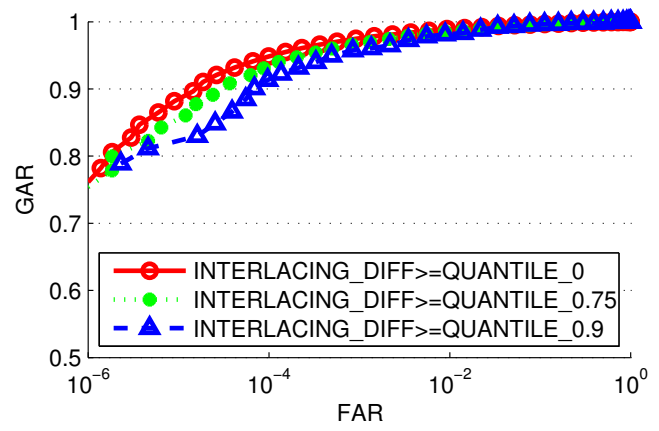

(a)

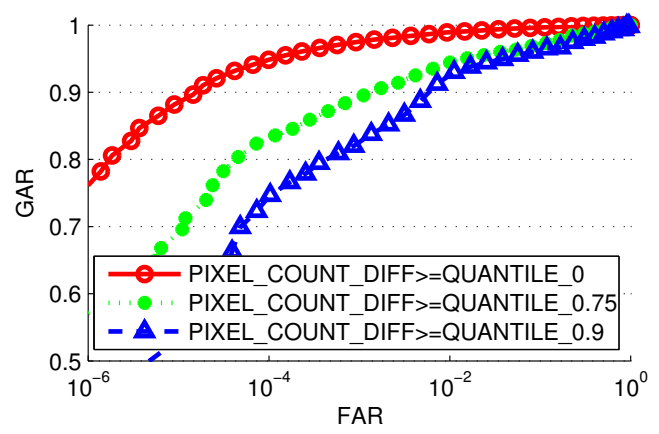

$(c)$

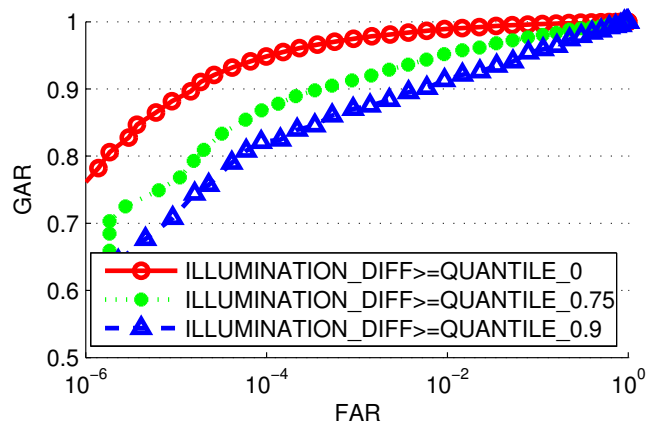

(b)

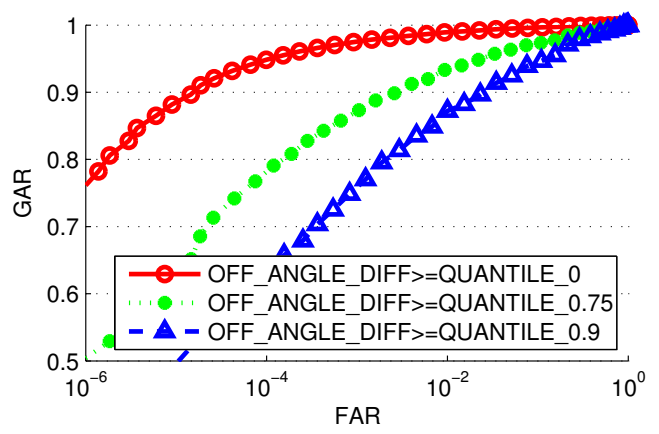

$(d)$

Figure 4.5: ROC curves for ICE2005 dataset (a) selecting matching scores using interlacing measure; (b) selecting matching scores using illumination measure; (c) selecting matching scores using pixel count measure; and (d) selecting images using off-angle measure.

\subsection{Conclusions}

This work proposed a number of new absolute and relative (global and local) quality measures for iris video. The performance of the proposed measures was evaluated by analyzing the relationship between the quality of iris images and verification performance of the system (in terms of ROC curves). These relationships indicate that proposed quality measures, when evaluated individually, do substantially influence recognition performance.

The importance of each individual quality factor, evaluation of the degree of their correlation and designing a super-combination rule for the proposed factors is the ongoing work in our lab [75]. 


\section{Chapter 5}

\section{Iris Segmentation}

Since segmentation of iris images is one of the most important preprocessing steps performed by an iris recognition system, we devote this entire Chapter to the design and analysis of a robust iris segmentation method. Iris biometric is one of the most reliable biometrics with respect to performance. However, this reliability is a function of the ideality of the data. One of the most important steps in processing non-ideal data is reliable and precise segmentation of the iris pattern from remaining background. In this chapter, a segmentation methodology that aims at compensating various nonidealities contained in iris images during segmentation is proposed. The virtue of this methodology lies in its capability to reliably segment non-ideal imagery that is simultaneously affected with such factors as specular reflection, blur, lighting variation, occlusion and off-angle images. We demonstrate the robustness of our segmentation methodology by evaluating ideal and non-ideal datasets, namely Chinese Academy of Sciences iris data version 3 Interval subdirectory (CASIA III INT) [76], Iris Challenge Evaluation (ICE) data [74], West Virginia University (WVU) data [77], and WVU Off-angle data [78]. Furthermore, we compare our performance to that of our implementation of Camus and Wildes's algorithm [79], and Libor Masek's algorithm [41]. We demonstrate considerable improvement in segmentation performance over the former mentioned algorithms. 


\section{$5.1 \quad$ Introduction}

Current iris recognition algorithms that have potential for high recognition performance require highly constrained subject presentation. When this constraint is removed or unavailable, such as in surveillance applications or "iris at a distance", the quality of the data may be degraded by heterogenous factors [80]. These factors, such as specular reflection, lighting variation, blur, off-angle, and occlusion negatively impact segmentation and recognition performance as verified by Kalka et al [70], [69] and Chen et al [64] in their works on iris image quality.

Classically, pupil and iris regions have been localized using efficient integro-differential operators as defined by Daugman [46]. This operator remains in use today for the majority of commercial systems. Wildes [81] introduced pupil and iris localization by use of edge detection which is followed by a circular Hough transform. A number of recent approaches, based on variants of the former methodology that utilize the Hough transform have been developed (for example, [74,82], [83]). Other algorithms employ morphological operators [84] and ellipse fitting $[85,86]$ to cope with the non-circular shape of the pupil/iris. One limitation concerning these algorithms is that design and testing was developed for ideal data. This may result in significant degradation of performance when the algorithms are applied to non-ideal imagery.

There has been some work focusing on non-ideal iris, specifically off-angle [22,87], or less constrained environments [6]. However, these works mostly focus on bringing off-angle iris images into frontal view in order to involve iris recognition algorithms designed for frontal view ideas irises, rather than dealing with the segmentation aspects related to this factor. Proença and Alexandre [80] identify a more noise tolerant feature set, consisting of pixel location and intensity, which is then used to construct an edge map followed by a circular Hough transform for localization. They demonstrate good results when evaluating the UBIRIS dataset [7]. One disadvantage with this approach is that inexact localization can result from off-angle images because of the circular fit. There are many other publications that focus on particular aspects related to nonideal iris and processing of nonideal iris images. We placed them in three broad categories: (1) unique approaches (for example, [88], [89] 
and [90]. The authors of the latter paper claim their optimal partitioning based algorithm is robust to poor illumination, blur, occlusion and eye glasses; (2) papers dealing with estimation of occlusions (for example, [91], [92], [93]); (3) papers that are dealing with specular reflections (for example, [91], [93]). The most recent trend in designing robust segmentation techniques is to apply nonparametric approaches such as active contours to describe the iris and pupil boundaries [94,95]. In [95], besides active contour fitting on the boundaries and generalized coordinates, new off-angle image solution called "Fourier based trigonometry", a statistical eyelash detection method, and a score normalization method are introduced.

In this work, we propose a methodology for automatic segmentation of iris images that sequentially compensates non-ideal factors present in poor quality iris images. This work is inspired by our previous (see [70] and [69]) and current work on iris image quality. Iris image quality is determined by a set of quality factors including motion and defocus blur, contrast, unbalanced illumination, occlusion, off-angle, and other factors. Factors are estimated for each iris image individually. A separate procedure to evaluate a specific quality metric is designed. The final result is presented both in the form of a vectors of individual quality values and in the form of a single number obtained by applying Dempster-Shafer combination criterion [96]. The fact that quality metrics can be estimated individually laid a foundation for this work.

This chapter describes a sequence of procedures and steps that are intended to compensate detected nonidealities in iris images in order to successfully segment nonideal iris images. The following compensation steps are applied. Similar to many other segmentation methods we first detect and eliminate specular reflections through the application of hard thresholding and Partial Differential Equation (PDE)-based inpainting, respectively. Then we localize the pupil by invoking diverse information available in the image involving pupil location, intensity, and shape. We further propose a technique to eliminate occlusions resulting from the overlap of long eyelashes with the area of the pupil. This step ultimately influences segmentation of the pupil area, which is performed next. Both the pupil and iris in an iris image are segmented by fitting a rotated ellipse, an ellipse parameterized by the parameters of main axes, center point, and the angle of rotation. During the iris segmenta- 
tion, we apply a contrast balancing procedure to ensure correct segmentation of bright and shadowed portions of the iris. Simultaneously with iris boundary we estimate the occlusion mask by applying an edge detector and slightly smearing the mask to ensure connected edges.

The rest of this chapter is organized as follows. Section 5.3 describes our segmentation methodology for a pupil and iris localization. This section also lists preprocessing steps required for successful segmentation in the presence of non-idealities. Section 5.4 illustrates the performance of our segmentation algorithm using non-ideal data such as CASIA III INT (CASIA for Institute of Automation, Chinese Academy of Sciences) [76], ICE [71,74], WVU [77] and WVU off-angle datasets [78]. Finally, a summary and conclusions are presented in Section 5.5.

\subsection{Effects of Degradations on Iris Segmentation}

In this section we empirically demonstrate how various degrading factors influence iris image segmentation. We manually select a number of images with strong degradations due to a single factor. Selected images are segmented using our robust segmentation technique designed to deal with degraded iris images [58]. Fig. 5.1-5.8 provide examples of degraded images and the results of their segmentation. Images are selected from four large public iris datasets: Chinese Academy of Sciences iris dataset version 1, 2 and 3 (CASIA) [76], Iris Challenge Evaluation (ICE) dataset [74], West Virginia University (WVU) dataset [77], and WVU Off-angle dataset [78]. Each figure presents two iris images, a relatively clean (ideal) image and a strongly degraded image, but mainly by a single degrading factor. Typically, an image with no or light degeneration is segmented perfectly. The other image characterized by strong degeneration due to a single factor cannot be well segmented. A subjectively evaluated ground truth for fair or incorrect segmentation is provided using dotted line. 

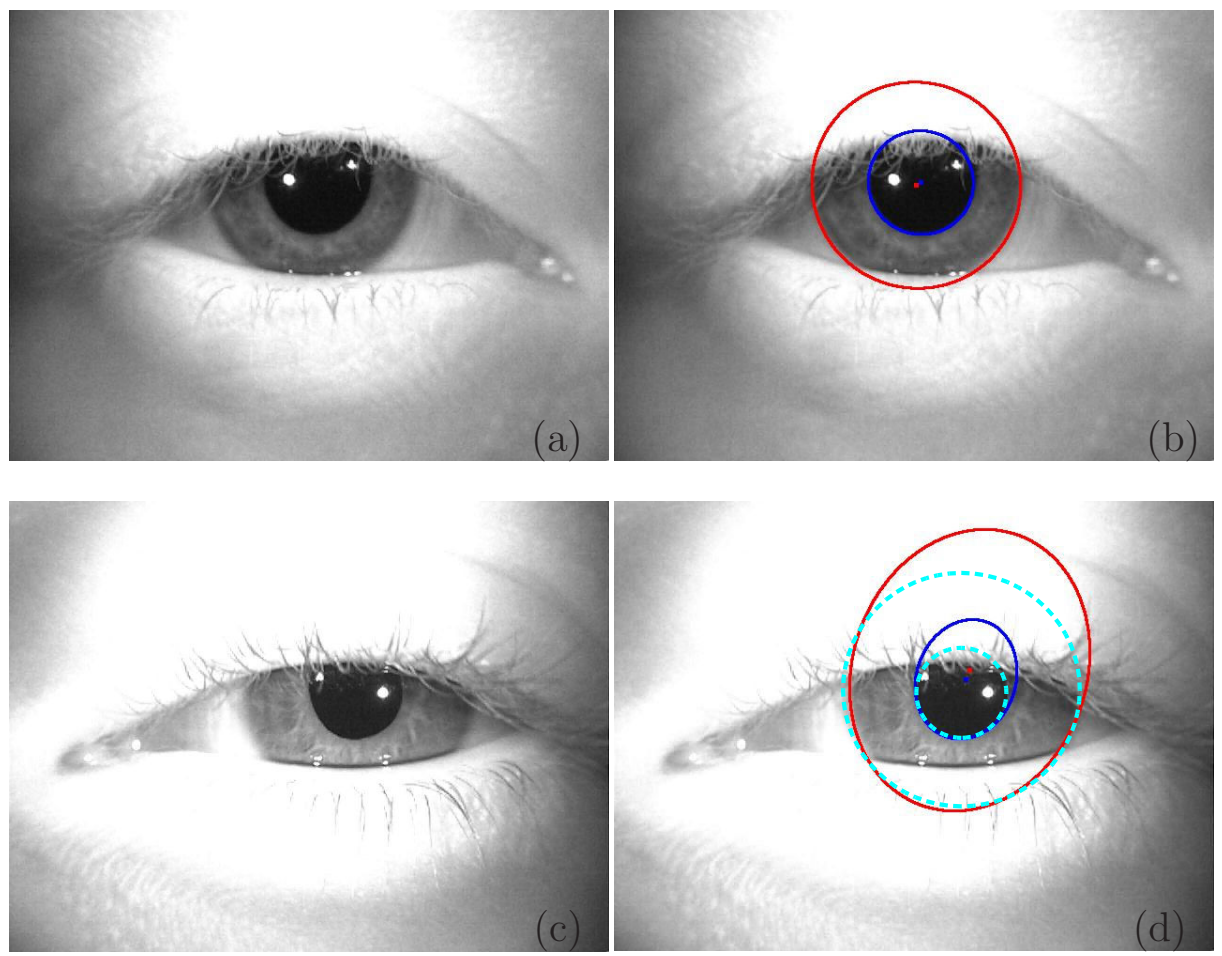

Figure 5.1: Segmentation of occluded iris images: (a) the original image $I_{1}$ (from WVU dataset), (b) correct segmentation for $I_{1}$, (c) the original image $I_{2}$ (from WVU dataset), (d) incorrect segmentation of $I_{2}$. The occlusions are due to eyelids.

Fig. 5.1 illustrates the degradation due to eyelid occlusion. Note that the second image $I_{2}$ is not segmented well due to dominating occlusion of a large portion of pupil and limbic boundaries.

In Fig. 5.2 through Fig. 5.8, pairwise examples are images of the same iris acquired under different conditions. Fig. 5.2 demonstrates another type of occlusion caused by eyelashes. In the second image $I_{2}$, the contrast between iris and sclera is very low. In addition, very long eyelashes form a "fake limbic boundary," which results in incorrect segmentation.

As illustrated in Fig. 5.3, strong specular reflections may damage the line of the pupil boundary. In this example, the damaged pupil area is relatively large compared to the size of the pupil. Not only specular reflections but also low contrast (Fig. 5.4) and poor illumination (Fig. 5.5) may contribute to the problem of estimating the pupil boundary.

In the examples illustrated in Fig. 5.4 and Fig. 5.5, careful visual evaluation of the images may result in a carefully evaluated ground truth. In the following two examples presenting 

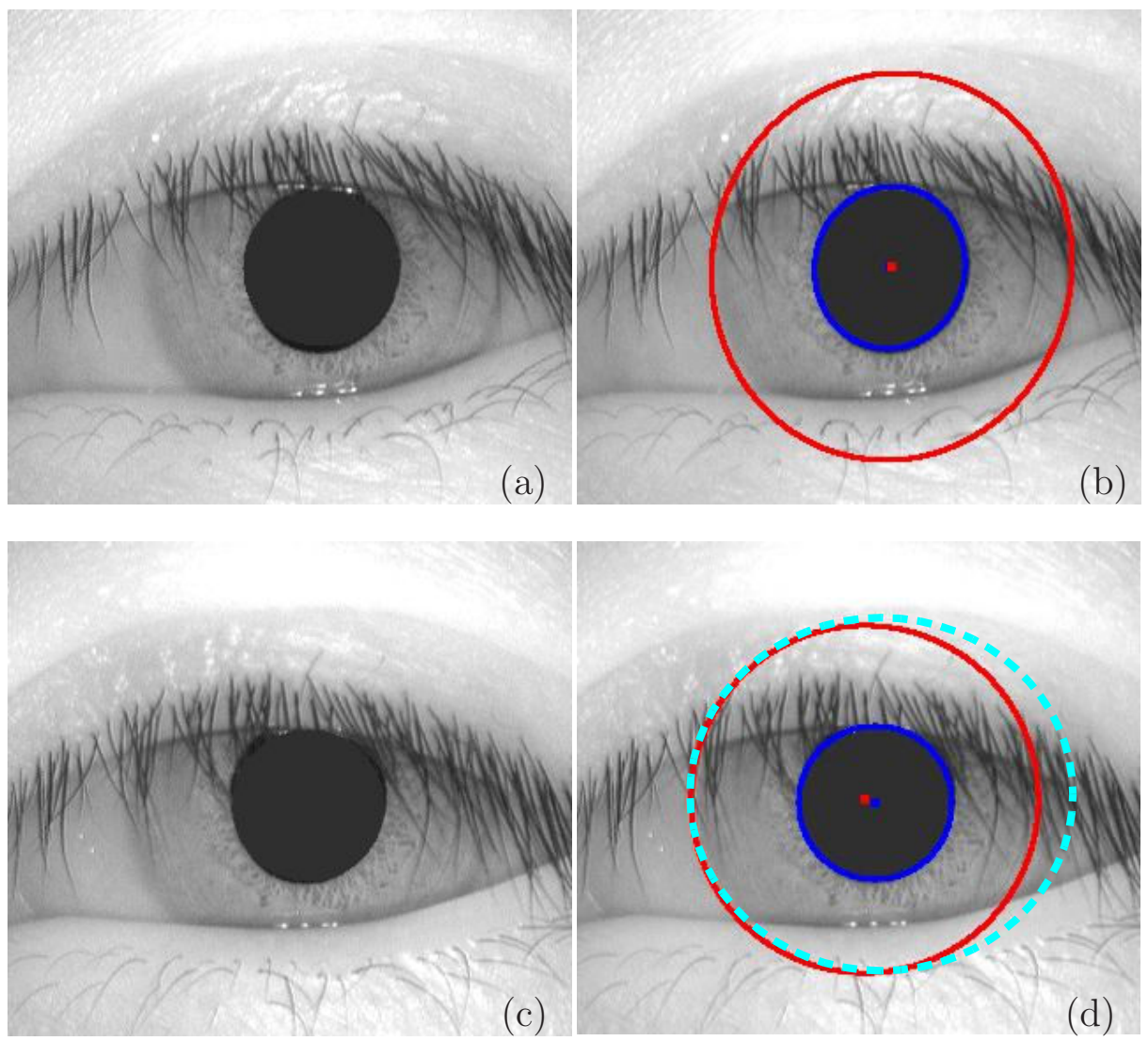

Figure 5.2: Segmentation of occluded iris images: (a) the original image $I_{1}$ (from CASIA I dataset), (b) correct segmentation of $I_{1}$, (c) the original image $I_{2}$ (from CASIA I dataset), (d) incorrectly segmented $I_{2}$. The occlusions are due to eyelashes.

the cases of strong out-of-focus (Fig. 5.6) and motion (Fig. 5.7) blurs, no segmentation ground truth can be found. The subjective judgement in these cases does not allow to state that the visually best choice is the best choice in recognition sense.

The last example presents the case of the off-angle images (Fig. 5.8). This example illustrates that iris images with a strong off-angle may cause a large error in estimating the angle of rotation, provided that an ellipse fitting procedure is adapted to find the limbic boundary.

In the presence of strong degradations, the most robust segmentation algorithm will fail to produce a correct result. Therefore, after the segmentation an automatic iris recognition system has to perform evaluation of the quality of segmentation in order to rule out images with failed segmentation. The examples above illustrate that incorrect segmentation leads to 

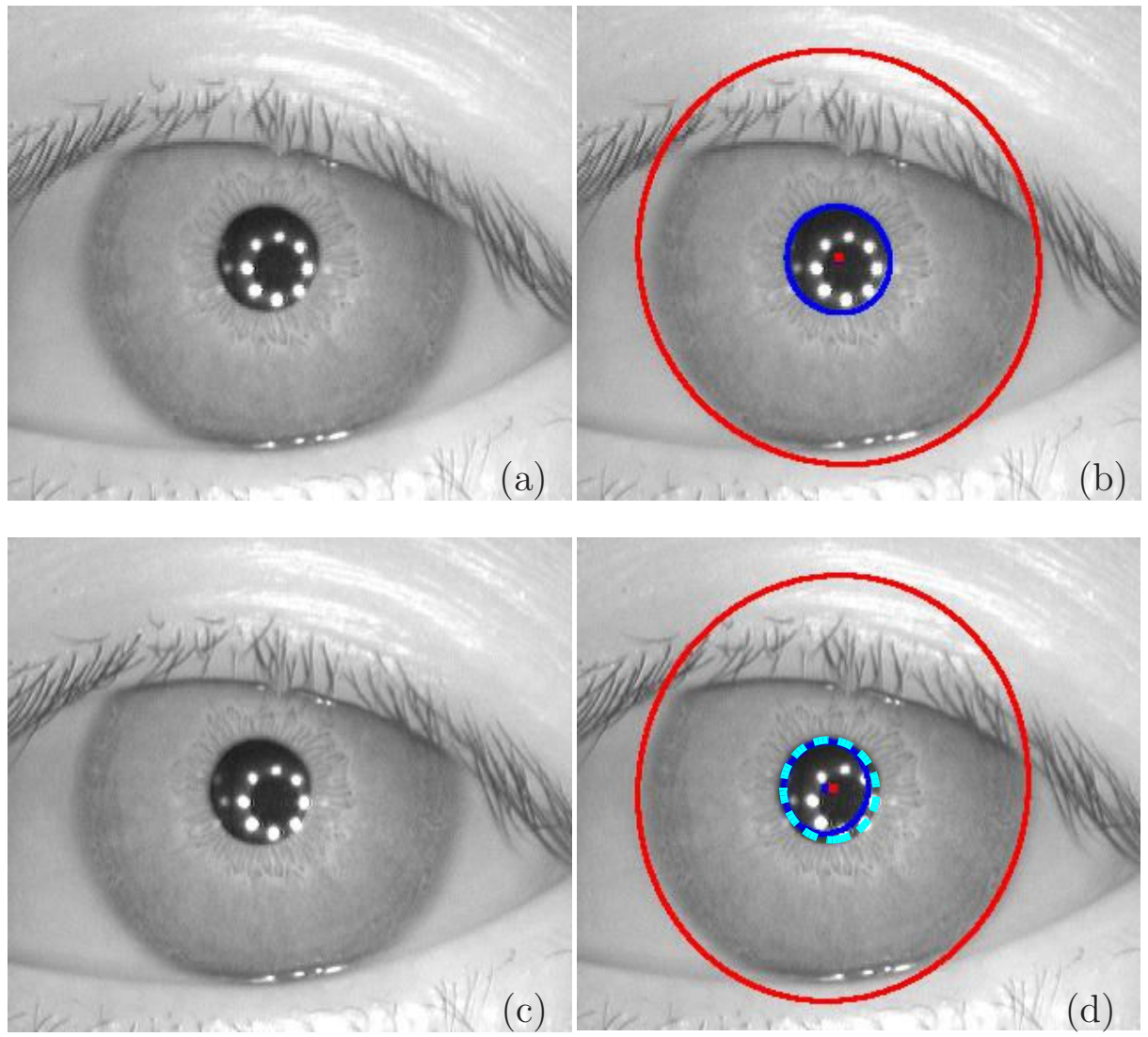

Figure 5.3: Segmentation of iris images containing strong specular reflections: (a) the original image $I_{1}$ (from CASIA III dataset), (b) correct segmentation of $I_{1}$, (c) the original image $I_{2}$ (from CASIA III dataset), (d) poor segmentation of $I_{2}$ due to the presence of strong specular reflections.

a certain offset between the true boundary and the estimation. In most cases, no continuous clear edges exist that follow incorrectly estimated boundaries. If both the pupil boundary and the limbic boundary are almost correctly estimated, then there must be long clear edges observed along the unaffected boundaries. Therefore, the edges along the estimated boundaries can be used to evaluate the precision (accuracy) of iris segmentation.

\subsection{Segmentation Methodology}

Traditionally, iris segmentation algorithms perform a number of steps. Ours includes: (A) preprocessing; (B) pupil segmentation; (C) iris segmentation; (D) occlusion estimation and (E) unwrapping. A general block-diagram of the steps in the proposed segmentation 

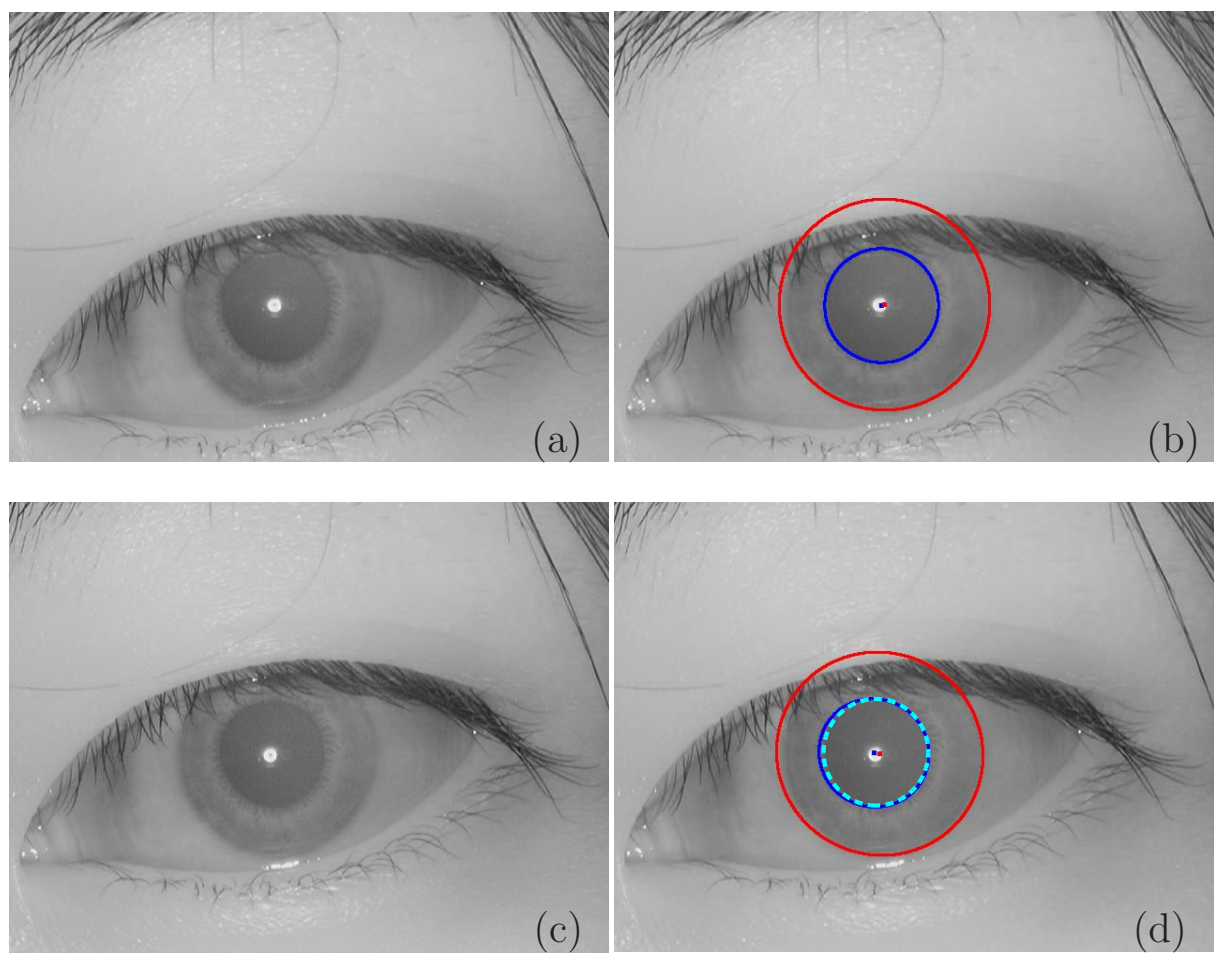

Figure 5.4: Segmentation of images with low contrast: (a) the original image $I_{1}$ (from CASIA II dataset), (b) correctly segmented $I_{1}$, (c) the original image $I_{2}$ (from CASIA II dataset), (d) fair segmentation of $I_{2}$.

algorithm is shown in Fig. 5.9. Although some of those steps appear to be similar to steps in traditional iris segmentation algorithms, each step in Fig. 5.9 targets compensation of a specific nonideality and includes a procedure for compensation of the nonideality in addition to traditional processing. The sections below provide details of each step.

\subsubsection{Preprocessing}

The preprocessing of iris images consists of detection of specular reflections, their removal and denoising of images.

\section{Inpainting of Specular Reflections}

Due to high intensities of specular reflections and large gradient values along their boundaries, if not successfully removed, specular reflections substantially degrade performance of an intensity- and gradient-based segmentation algorithm. The details of preprocessing are 

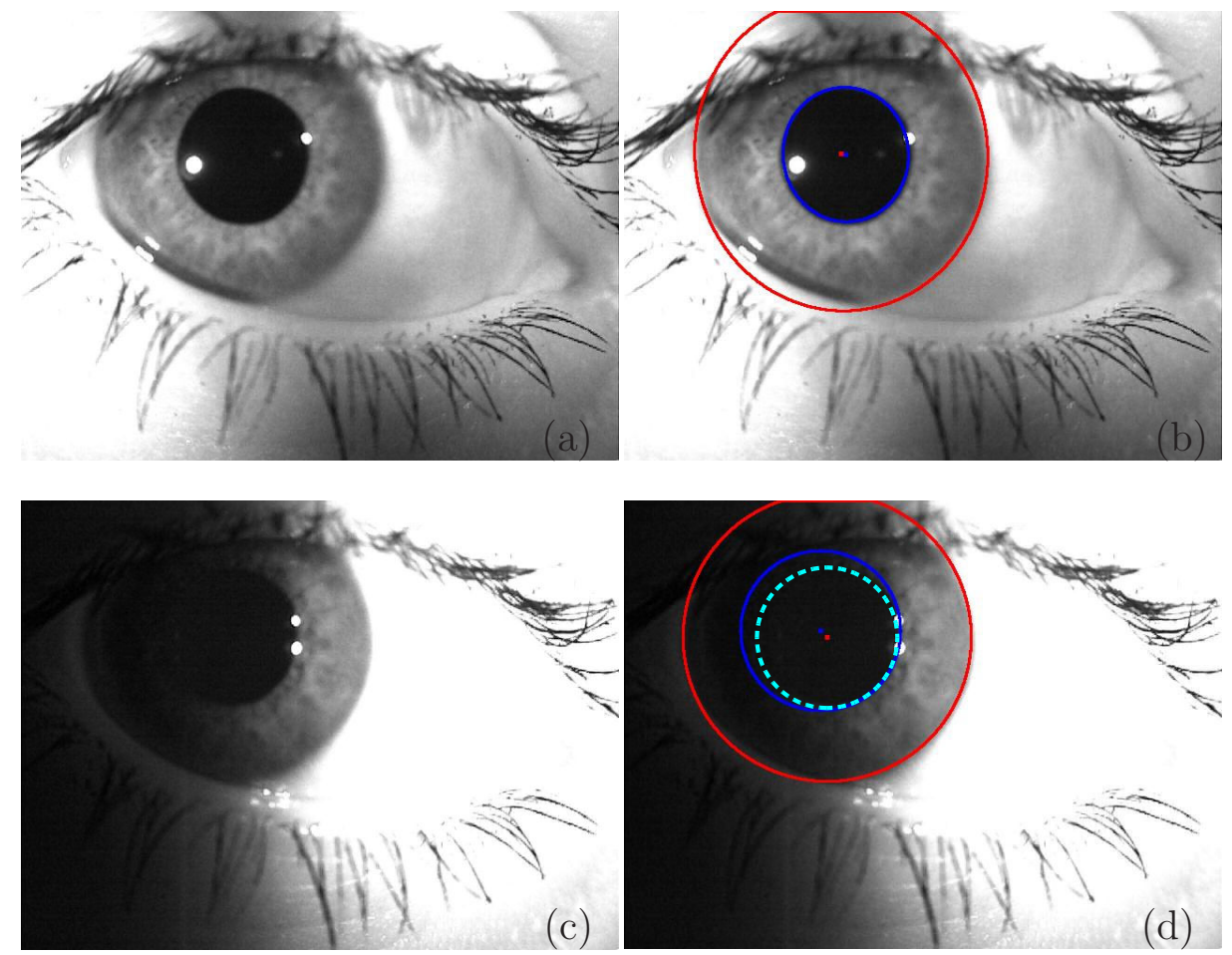

Figure 5.5: Segmentation of unevenly illuminated images and images with low contrast: (a) the original image $I_{1}$ (from WVU dataset), (b) correct segmentation of $I_{1}$, (c) the original image $I_{2}$ (from WVU dataset), (d) incorrect segmentation of $I_{2}$.

provided below.

Let $A\left(n_{1}, n_{2}\right)$ be an image intensity at a location $\left(n_{1}, n_{2}\right)$. In our approach specular reflections are localized through application of a thresholding function, $\gamma\left(n_{1}, n_{2}\right)$. An intensity value of a pixel $\left(n_{1}, n_{2}\right)$ is considered as a specular reflection if the intensity value $A\left(n_{1}, n_{2}\right) \geq \gamma\left(n_{1}, n_{2}\right)$. For specular reflections within the area of the pupil, a threshold of value $\gamma_{1}$ is used. The value of this threshold influences the result of inpainting, which is important for the intensity-based pupil area selection described in the following subsection. For specular reflections inside of the iris area, a threshold $\gamma_{2}>\gamma_{1}$ is used to avoid attributing well illuminated iris areas to specular reflections. To identify a potential pupil area we introduce another threshold $\tau$. In our implementation, $\gamma_{1}$ and $\tau$ are assigned the same value. 

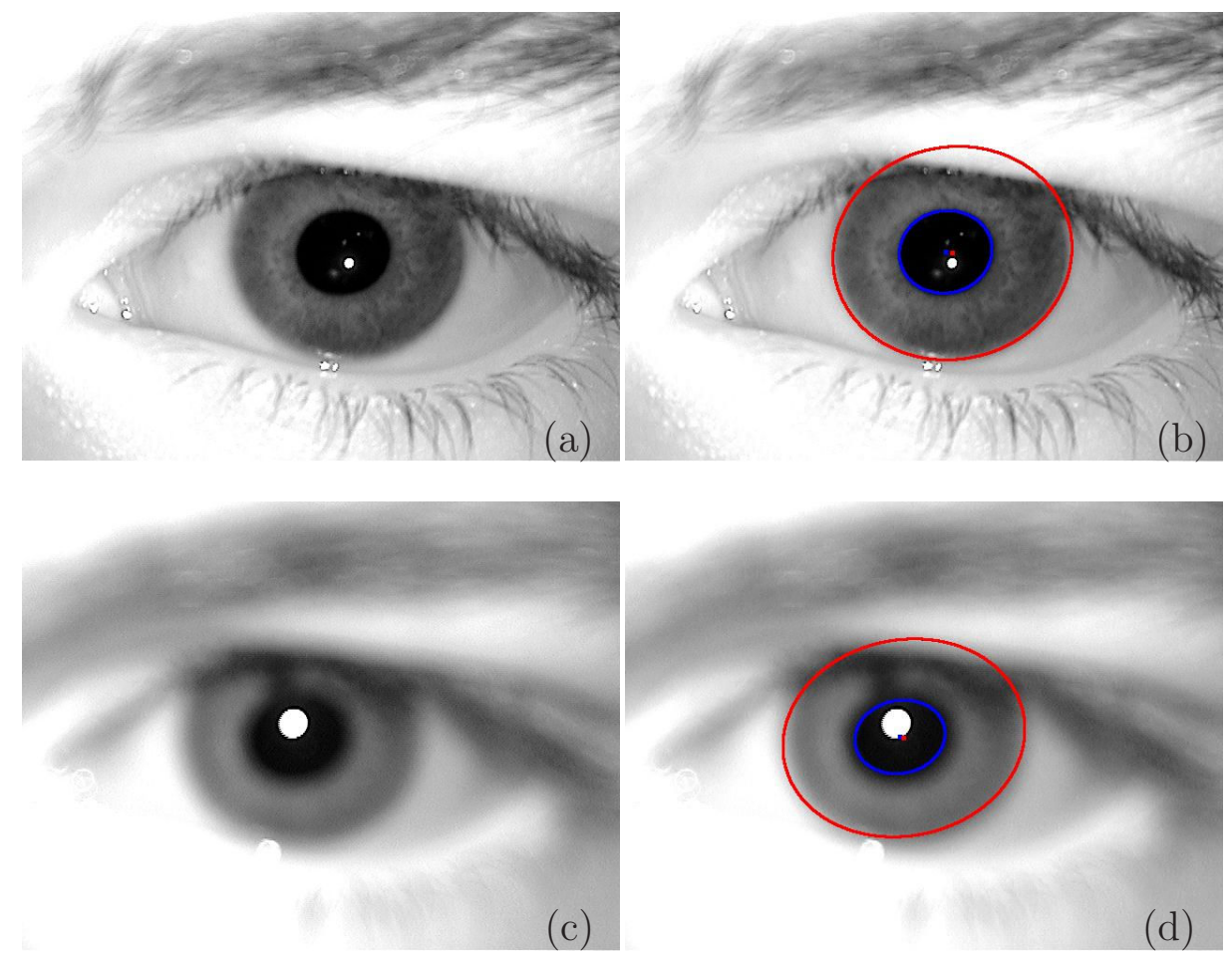

Figure 5.6: Segmentation of out-of-focused images: (a) the original image $I_{1}$ (from ICE dataset), (b) segmentation results for $I_{1}$, (c) the original image $I_{2}$ (from ICE dataset), (d) poor segmentation of $I_{2}$.

Therefore, the final thresholding function $\gamma\left(n_{1}, n_{2}\right)$ is described as:

$$
\gamma\left(n_{1}, n_{2}\right)=\left\{\begin{array}{l}
\gamma_{1} \text {, pixel }\left(n_{1}, n_{2}\right) \text { is located inside of } \\
\text { the potential pupil area determined by } \tau, \\
\gamma_{2}, \text { otherwise, }
\end{array}\right.
$$

where parameters $\gamma_{1}, \gamma_{2}$ and the maximum pupil intensity value $\tau$ have to be preset. These parameters depend on the type of the sensor used to acquire images and the environment. The procedure results in a rough masking of the regions of specular reflections. Localized specular reflections are further refined based on the shape and connectivity properties of masked areas. Although a set of tuned parameters used to process data to demonstrate performance of the proposed segmentation algorithm in Sec. 5.4 is suitable for a number of iris capture devices operating in near-IR range, our specular reflection detector has to be recalibrated, that is, $\gamma_{1}, \gamma_{2}, \tau$ have to be evaluated, if new capture devices or new environmental set ups are used. The parameters can be easily estimated from a small set of sample iris images when capture device or environmental setup change. 

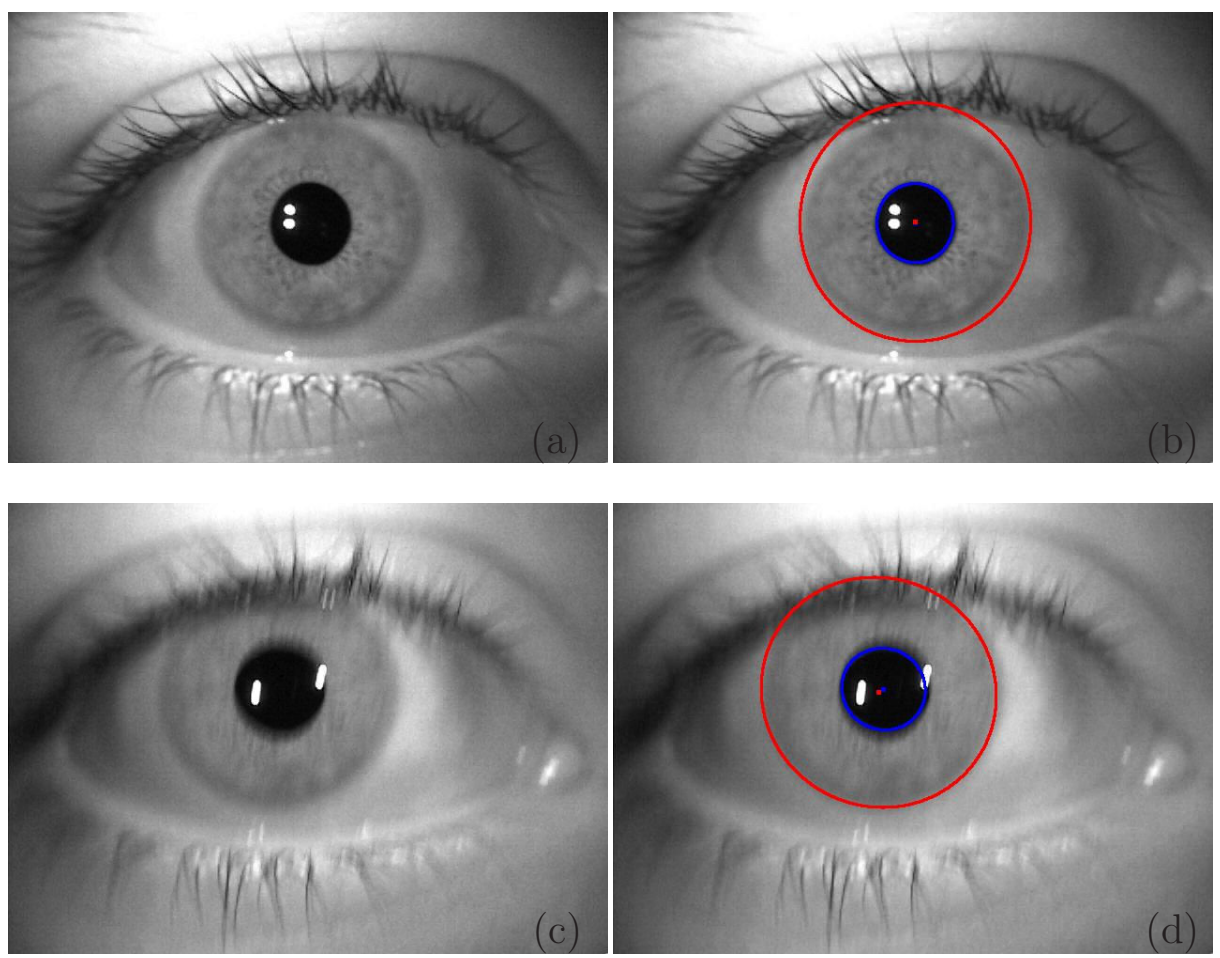

Figure 5.7: Segmentation of motion blurred iris images: (a) the original image $I_{1}$ (from WVU dataset), (b) segmentation result for $I_{1}$, (c) the original image $I_{2}$ (from WVU dataset), (d) fair segmentation result for $I_{2}$.

Once specular reflections are detected, they have to be treated individually based on their location in the image. A special care has to be taken of the specular reflections located inside the pupil. For this purpose, the specular reflections located inside of the possible pupil regions are inpainted first. To compensate for the missing data, a sparse partial differential equation (PDE)-based inpainting procedure [97] is applied to refined localized specular reflections. After inpainting, additional intensity adjustment needs to be integrated to ensure that the inpainted areas have similar intensity values as unaffected pupil areas. To adjust the intensity value, possible unaffected pupil regions have to be detected. It is assumed that they are close to the specular reflections but have a relatively low average intensity value. Once the unaffected pupil regions are detected, 0.1 quantile and 0.5 quantile intensity values of various unaffected pupil regions are used to upper and lower bound the intensity values of inpainted regions.

The complete inpainting procedure includes the following steps: 

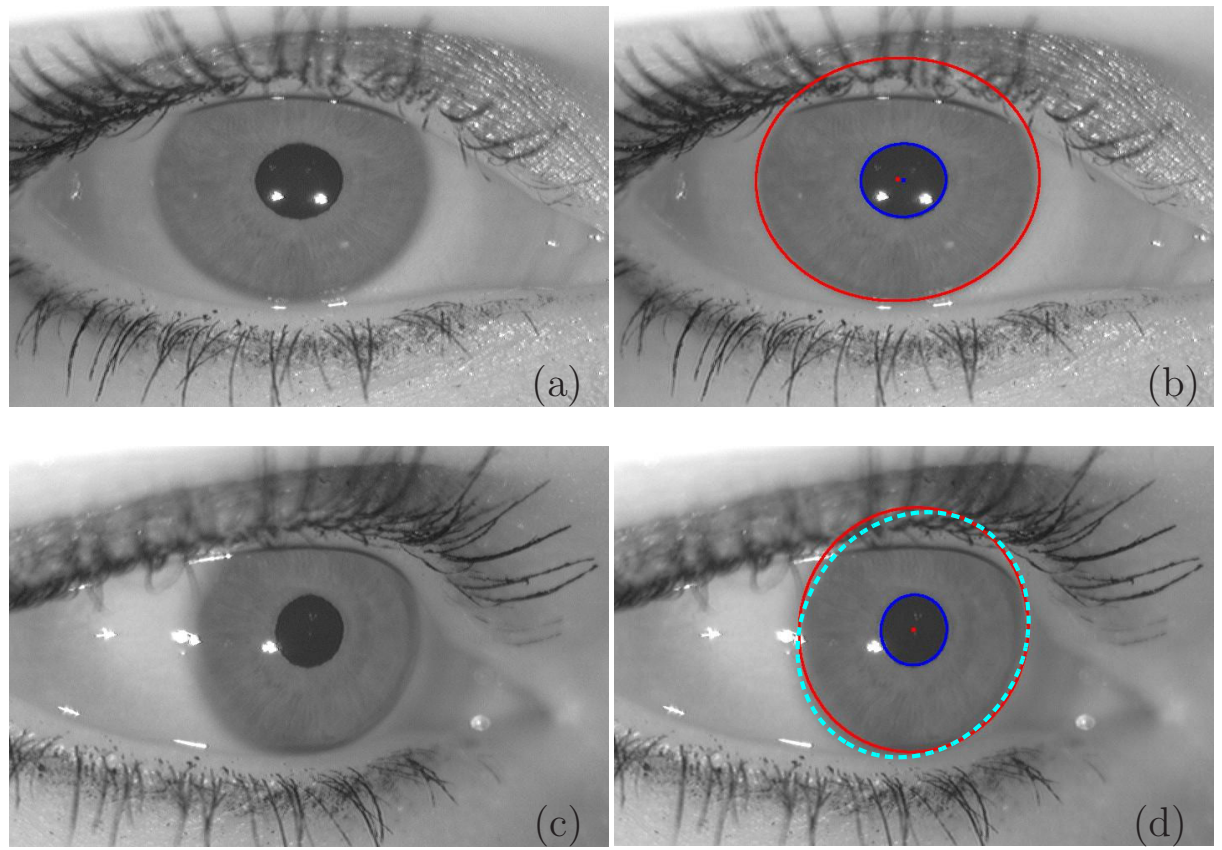

Figure 5.8: Segmentation of the off-angle iris: (a) the original frontal view image $I_{1}$ (from WVU Off-angle dataset), (b) segmentation result for $I_{1}$, , (c) the original 30 degree off-angle image $I_{2}$ (from WVU Off-angle dataset), (d) fair segmentation result for $I_{2}$.

- A potential pupil area, $\operatorname{mask}_{p 1}$, is identified using the maximum value of pupil intensity, $\tau$, as a threshold. An enlarged mask mask $k_{22}$ is obtained after closing unfilled regions.

- Potential regions containing specular reflections, mask $_{s}$, are selected using the thresholding procedure (6.1). The regions are further refined using relationship between neighboring points. Specular reflection areas are selected using a threshold $\gamma$.

- The mask $_{s}$ is dilated to include all possible affected regions.

- The intersection of mask $_{p 2}$ and mask $_{s}$ is selected to be inpainted region inside of the pupil denoted as $\operatorname{mask}_{p 3}$.

- The area mask $_{p 3}$ is inpainted, and the intensity values in the region are adjusted using the intensity information of the region mask $_{p 1} \bigcap$ mask $_{s}$, the intersection of mask $_{p 1}$ and mask $_{s}$.

- The region of potential specular reflections, mask $_{s 1}$, is updated by applying the thresholding procedure (6.1) to the inpainted image. The resulting mask is again refined using 


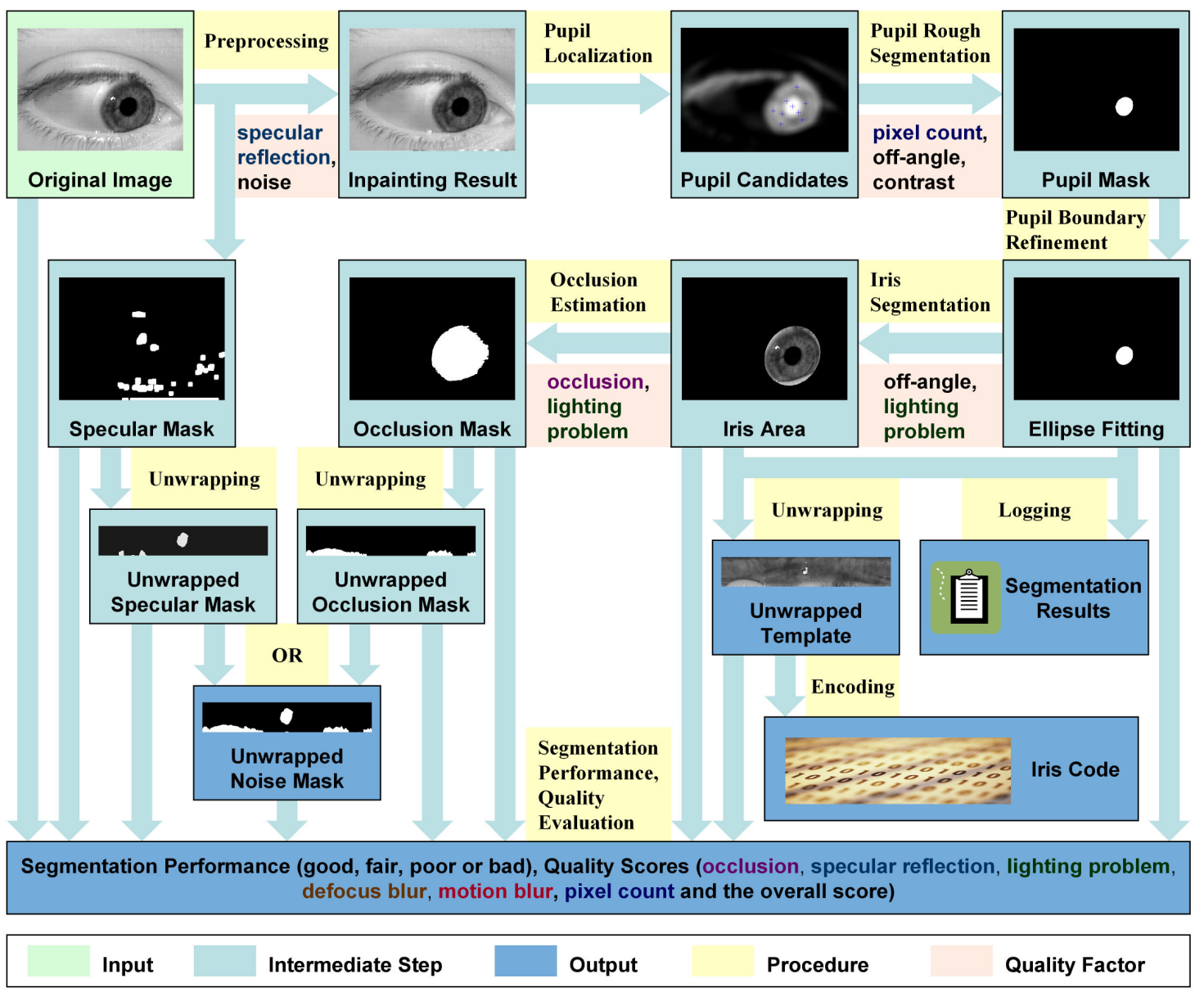

Figure 5.9: Block diagram of the iris segmentation procedure. 
the relationship between neighboring points.

- The mask $_{s 1}$ is expanded to include all possible affected regions.

- The region selected by mask $_{s 1}$ is inpainted.

An example of the inpainting process is shown in Fig. 5.10. The purpose of inpainting is

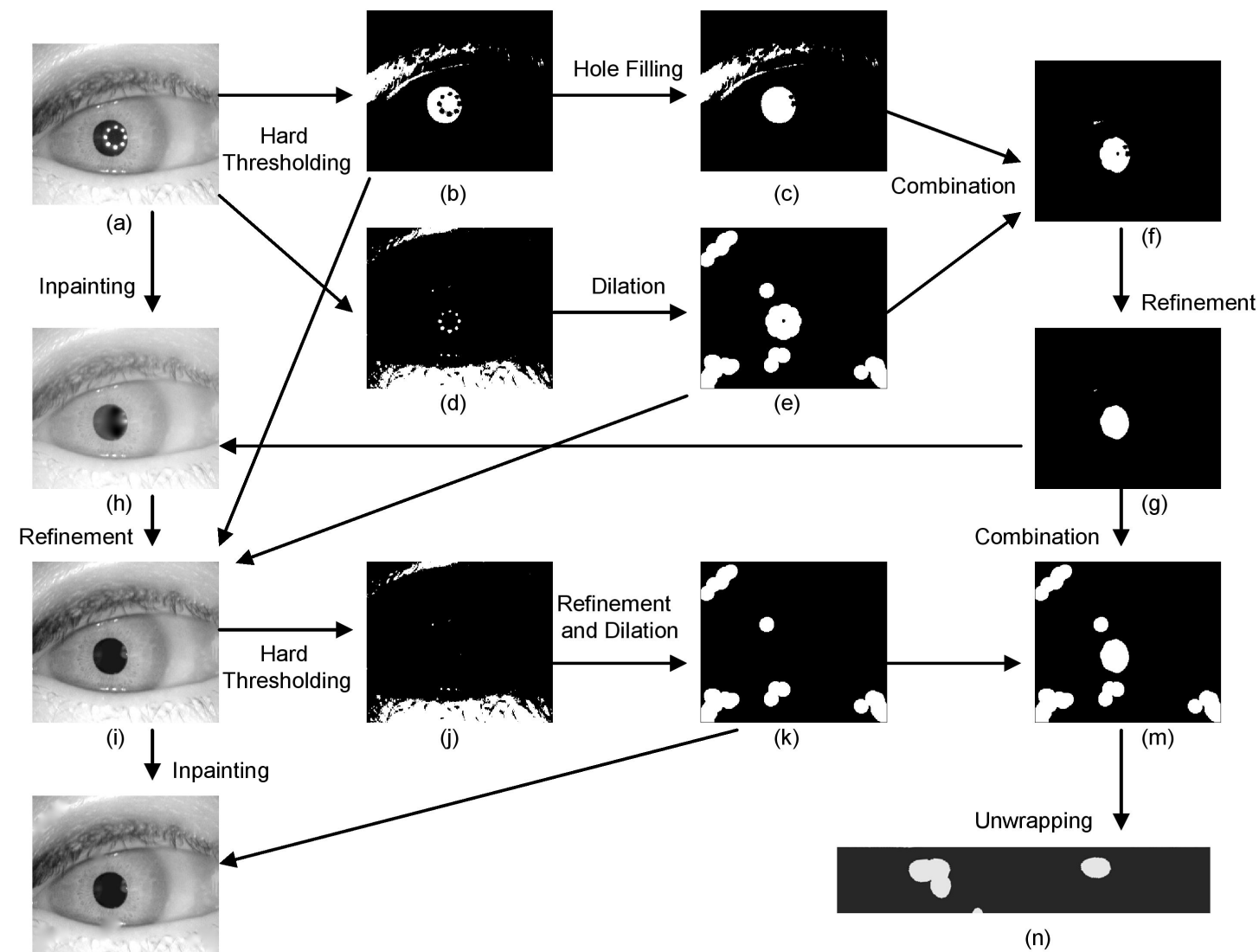

(I)

Figure 5.10: Inpainting steps: (a) the original image, (b) mask $k_{p 1}$ of the potential pupil area, (c) gaps in mask $_{p 1}$ are filled to obtain the mask mask $k_{p 2}$, (d) rough specular reflection mask is obtained using the threshold $\gamma$, (e) expanded specular reflection mask $k_{s}$, (f) combination of (c) and (e), (g) the intersection mask $_{p 2} \bigcap$ mask $_{s}$ of two masks, (h) result of initial inpainting, (i) refined inpainting result after intensity adjustment, $(\mathrm{j})$ rough specular reflection mask $k_{s 1}$ obtained using the threshold $\gamma$ applied to $(\mathrm{i}),(\mathrm{k})$ refined and expanded specular reflection mask $_{s 1}$, (l) final result of inpainting, (m) final specular reflection mask used to generate the template mask, (n) template mask.

not to make the image more pleasing, or to recover damaged information; it is intended to simplify the pupil segmentation procedure and increase a success rate of segmentation. 
An additional factor that can be detected and removed during the initial stage of preprocessing is the additive noise. Its influence on the iris boundary detection and estimation of occlusions is due to high absolute gradient values present in noisy images.

\section{Denoising}

To eliminate the effect of noise we choose to work with a two-dimensional adaptive Wiener filter. The values of the mean, $\mu$, and variance, $\sigma^{2}$, are estimated locally first for each pixel $\left(n_{1}, n_{2}\right)$ in the original image $A$ :

$$
\begin{gathered}
\mu=\frac{1}{N M} \sum_{n_{1}, n_{2} \in \eta} A\left(n_{1}, n_{2}\right), \\
\sigma^{2}=\frac{1}{N M} \sum_{n_{1}, n_{2} \in \eta} A^{2}\left(n_{1}, n_{2}\right)-\mu^{2},
\end{gathered}
$$

where $\eta$ is the $N$-by- $M$ local neighborhood of each pixel in the image A. In our current implementation $N$ and $M$ are set to 5 . Then the denoised image $B$ is given by:

$$
B\left(n_{1}, n_{2}\right)=\mu+\frac{\sigma^{2}-v^{2}}{\sigma^{2}}\left(A\left(n_{1}, n_{2}\right)-\mu\right) .
$$

where $v^{2}$ is the average of all locally estimated variances.

The result of noise removal is especially noticeable in low resolution images (for instance, images from CASIA I dataset). The noise removal often improves quality of unwrapped images.

After the preprocessing step is complete, the image is submitted for segmentation.

\subsubsection{Pupil Segmentation}

The pupil segmentation is composed of a number of steps. This includes (1) contrast stretching, a step that ensures clarity of the boundary between the pupil and iris; (2) smoothing step that removes extreme values without reducing sharpness of the image; (3) pupil localization that finds a potential location of the pupil; (4) rough segmentation of the pupil; (5) refinement of a roughly segmented pupil; (6) ellipse fitting into the pupil boundary; and (7) evaluation of precision of pupil segmentation. The rest of the section provides details of the steps above. 


\section{Contrast Enhancement (Normalization)}

Iris images acquired under nonideal experimental set up often have low contrast, especially along the boundary between pupil-iris and iris-sclera. To enhance the contrast we implement the following procedure:

$$
B\left(n_{1}, n_{2}\right)=\min \left(255, \frac{255\left(A\left(n_{1}, n_{2}\right)-\min (A)\right)}{\max \left(128, q_{0.4}(A)\right)-\min (A)}\right),
$$

where $A$ is the image before the contrast enhancement, $B$ is the image after the contrast enhancement, $\min ()$ is the function that returns the minimum value, $\max ()$ is the function that returns the maximum value, and $q_{x}()$ is the function that returns the $x$ quantile of image intensities [98].

Contrast enhancement is a necessary procedure that ensures the correct pupil segmentation. The noise and the other strong disturbances caused by the near pupil sphincter muscle and eyelashes are eliminated by performing a smoothing step described below.

\section{Smoothing}

To smooth the enhanced image we apply a median filter [99]. The value of an output pixel is determined by the median of the pixels in the neighborhood, rather than the mean. The median is much less sensitive to outliers compared to the mean. Median filtering removes these outliers without reducing the sharpness of the image. The size of the median filter depends on the resolution of the image to be smoothed. As the pupil segmentation procedure is intensity and gradient based, the smoothing of the image is important for the images with low contrast or with noisy pupil area. It also helps the detection of limbic boundary, since a large window median filter can remove misleading edges that can be observed inside of the iris area. This step may not be useful for good quality images, but does not alter performance in this case.

\section{Pupil Localization}

After image is enhanced and denoised, it is further subjected to pupil localization procedure. Two assumptions are made that are required to hold under both ideal and non-ideal settings: 
- The pupil area has the smallest intensity values in the image. Note that this assumption can be reversed in order to address the "red eye effect."

- The shape of the pupil can be described relatively well by a circle or ellipse.

The first assumption is valid under condition that the amount of the light reflected back from the pupil is relatively small. The light is typically reflected from the cornea's convex surface. This is valid under the ideal setting. The second assumption is valid both under ideal and non-ideal settings.

To locate the pupil we use two steps: (1) pupil candidates are selected based on the circular shape and (2) the best pupil candidate is selected based on intensity information and location with respect to the image center.

Potential candidates are selected by applying a circular Hough transform. When evaluating non-ideal data, eyelashes and strong specular reflections can introduce "fake" pupil candidates. A number of candidates can be removed by introducing a smoothing step prior to applying the Hough transformation.

After candidates have been selected, intensity and location information is used to find the optimal candidate. An adjusted intensity value for each candidate is generated based on another justifiable but relatively weak assumption: the pupil is normally located near the center of the iris images. The adjusted intensity value $w_{i}$ of a candidate $i$ is calculated based on convolved intensity value and its distance from the image center. Mathematically this is described as follows:

$$
w_{i}=\frac{I_{i}^{\prime}}{1+3 d_{i}^{2}},
$$

where $d_{i}$ is the distance between the candidate and the image center, and

$$
I^{\prime}=(255-I) * \mathbf{1}_{n \times n},
$$

is the result of the convolution of the inverted image $255-I$ with a square matrix of all ones of size $n \times n$. The value of $n$ is selected based on the size of the image. $I_{i}^{\prime}$ is the intensity value of a candidate at the location $i$. The candidate with the largest adjusted intensity is selected as the optimal candidate. Fig. 5.11 is an illustration of this process. Once the 

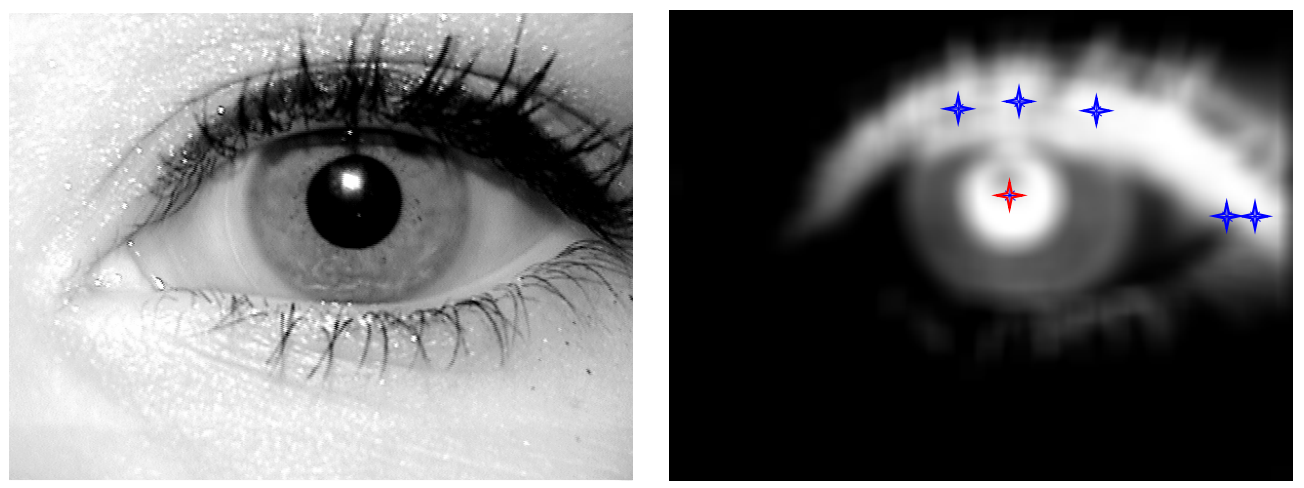

Figure 5.11: In this example, six pupil candidates are selected based on a circular shape of bright regions. Then the intensity information is used to select the optimal candidate with the largest intensity value.

optimal candidate is selected, the pupil can be roughly segmented using similarity of the intensity values.

In some cases, the assumptions stated earlier may not hold. The method described above will help to consistently select the true pupil candidate. When the true pupil candidate is located near the boundary, the candidate can be correctly selected if no other pupil like area exists (with low intensity and circular shape) within the image. In this case the true candidate will return the highest adjusted value $w_{i}$ in spite of a higher value of penalty $d_{i}$. The assumption that the shape of the pupil can be described relatively well by a circle or ellipse, may not hold true for heavily occluded iris images or images with strong off-angle. Again, the true pupil candidate can be correctly selected if no other pupil like area exists (with low intensity and circular shape). Circular Hough transform is tolerant to partial occlusions or imperfect circular boundaries. In this case the true pupil area will be among candidates for pupil center and will return the highest adjusted value $w_{i}$ due to the lowest average intensity of the surrounding area.

\section{Rough Segmentation of the Pupil}

A $5 \times 5$ neighborhood around the optimal candidate is used to identify the initial minimum and maximum intensities used for searching the pupil area. This intensity range is refined based on the rough estimation of the pupil shape and the gradient information along the region boundary. The pupil area is assumed to be relatively well described by a circular 
shape and have a clear boundary. We further present a summary of the evaluation criteria:

- The size of the selected area should be larger than a threshold. In our experiments, we use 100

- The average gradient value along the boundary has to be evaluated. The true pupil boundary returns the maximum gradient value.

- The difference between the original shape and the ellipse fitted into the area have to be less than a threshold, we use 13\%. This threshold may need tuning for some databases to achieve the best performance.

After the rough evaluation of the pupil boundary we perform a few processing steps to compensate for eyelash occlusion and specular reflections on the pupillary boundary.

\section{Refinement of the Pupil Boundary}

Eyelashes can introduce "spike" like shapes along the boundary region. These "spike" like regions can be removed by analyzing the boundary locally. Fig. 5.12 is an illustration of this procedure. The center of mass for the rough segmentation region is used as an approximation to the pupil center. Then the boundary is scanned to find possible "spikes". There are two kind of "spikes"; we call them "valley" type and "longhorn" type. The first type can be detected using the distance from the boundary points to the center. A valley is detected whenever the distance as a function of the orientation angle achieves a local minimum. The second type of "spikes" can be detected using angular information. Whenever the angle is decreasing first, then increasing and then decreasing again, the spike observed in this case is of "longhorn" type. Detected "spikes" are removed by using a large circle of radius equal to the twice the distance from the cutting point to the center. An example of "spike" removal is provided in Fig. 5.13. Note while the iris image in Fig. 5.13 is of relatively high quality in terms of iris texture, the iris area is heavily occluded by eyelashes that cause problems for an intensity based pupil segmentation.

In the case when dark long eyelashes cross the pupil area, the estimation of pupil center and the following boundary refinement will be incorrect. To remove the unwanted eyelashes, 


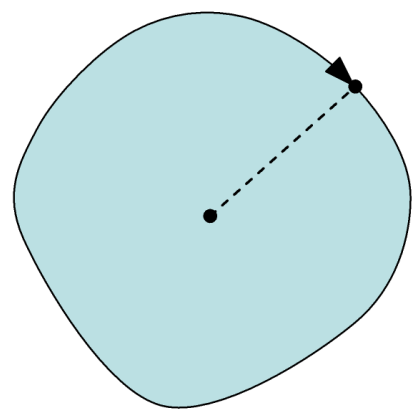

(a)

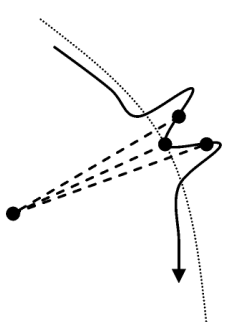

(b)

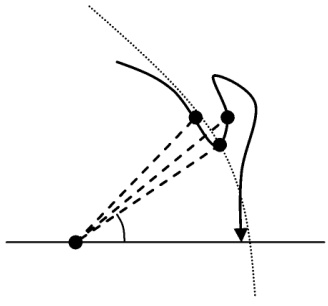

(c)

Figure 5.12: "Spike" removal: (a) scanning the boundary, (b) "valley" type "spike", (c) "longhorn" type "spike". Dotted curves in (b) and (c) are the boundaries of the circles that are used to remove "spikes."

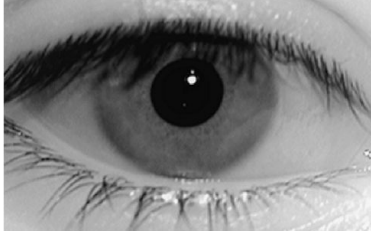

(a)

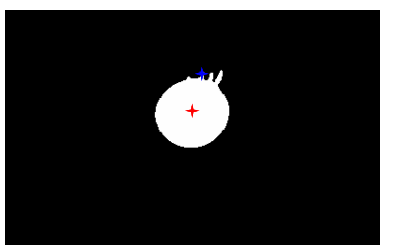

(b)

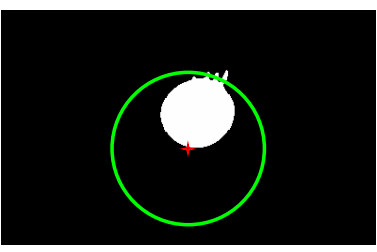

(c)

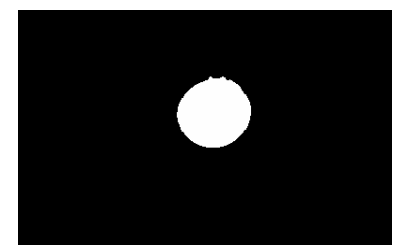

(d)

Figure 5.13: "Spike" removal: (a) the original image, (b) the estimated pupil boundary, (c) removing the "spike", (d) the refined boundary after the "spike" removal.

we manipulate the mask implementing dilation and erosion. The erosion breaks thin connections but it also affects other regions of the pupil. To compensate for the erosion, we dilate the area around the estimated pupil location. The dilated pupil mask is combined with the original mask to keep the original boundary instead of smoothed new boundary. The procedure is illustrated in Fig. 5.14.

Strong specular reflections along the pupil boundary or light colored eyelashes may cause the rough pupil boundary to become concave. We calculate the convex hull with respect to the pupil boundary in order to reduce this problem. An illustration of this process is provided in Fig. 5.15. As the pupil boundary affected by the specular reflections does not contain reliable information about the boundary, the following ellipse fitting procedure uses only unaffected pupil boundary. Unaffected pupil boundary can be easily selected using a mask of specular reflections formed during the preprocessing stage. 


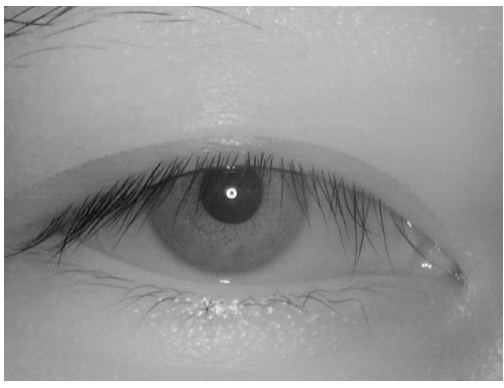

(a)

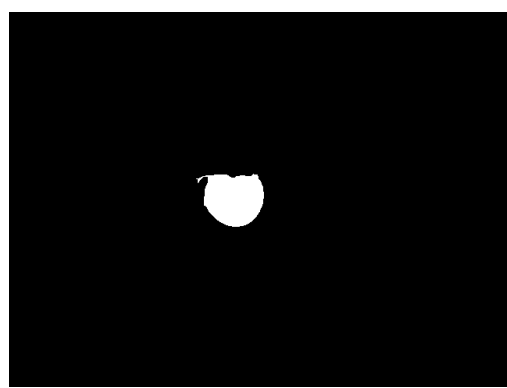

(d)

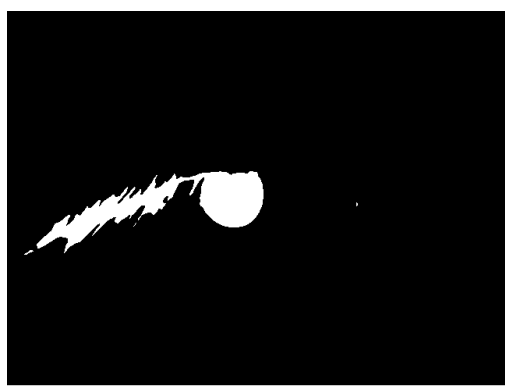

(b)

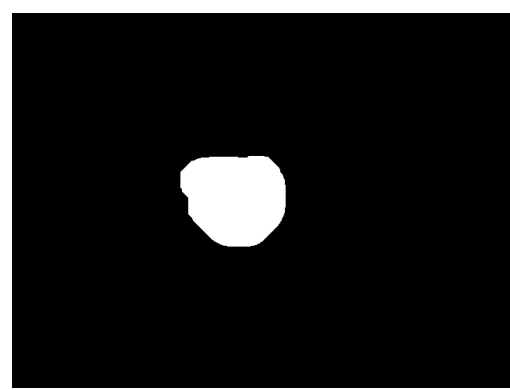

(e)

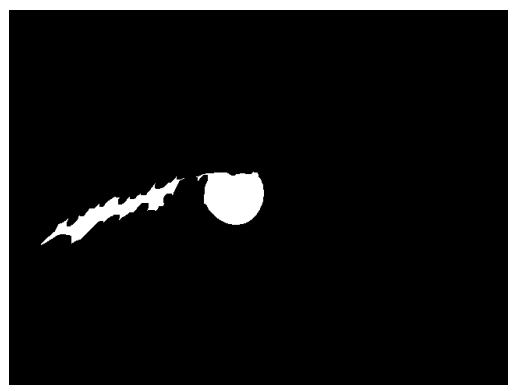

(c)

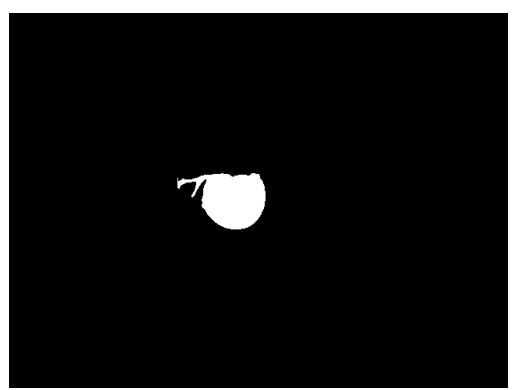

(f)

Figure 5.14: Breaking unwanted connections: (a) the original image, (b) the connected mask, (c) the erosion result, (d) select the pupil region from the eroded result using the location information of the optimal candidate, (e) the dilated pupil region, (f) the result of intersecting (a) and (e).

\section{Ellipse Fitting}

Once the boundary (or partial boundary) is determined, we apply a contour fitting procedure. There are several possible methods that can be used to accomplish the goal [46] [8], [81], [94], [100]. A graphical description of few available methods is provided in Fig. 5.16.

Classical iris segmentation techniques, such as Daugman's original segmentation algorithm [46], [8], assume a circular model for the pupil. That model has the advantage of being simple: only three parameters need to be estimated. The method provides relatively good results when applied to frontal view images. However, circular models do not describe the pupil boundary well if the iris is off-angle. In this case a more complex model is needed. For instance, an ellipse [81] can be used in place of the circle. This results in an additional parameter to evaluate. More complex models, such as b-spline-based or other nonparametric models [94] can be used to describe the boundary between the pupil and the iris. Those models, however, are sensitive to noise and occlusion if contour evolution is not well controlled. 


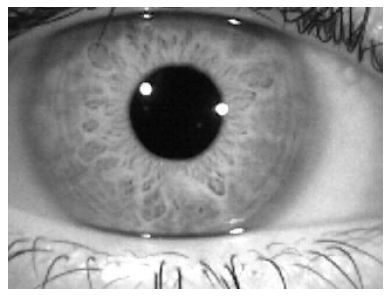

(a)

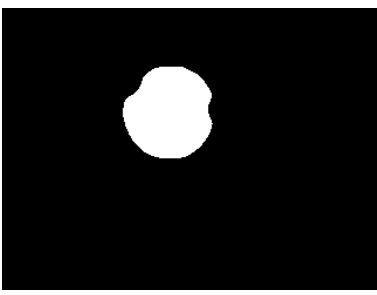

(b)

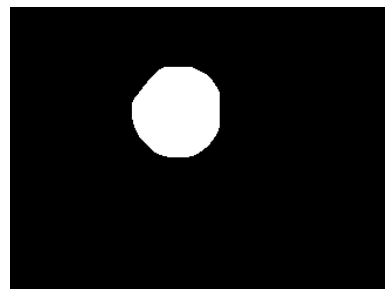

(c)

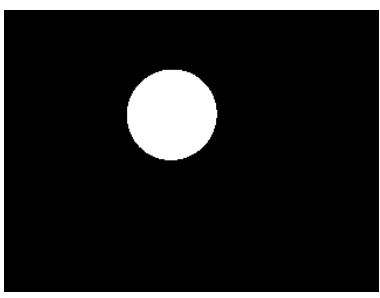

(d)

Figure 5.15: Concavity removal: (a) the original image, (b) the initial concave pupil mask, (c) the convex hull result, (d) the result of ellipse fitting procedure using only unaffected pupil boundary.

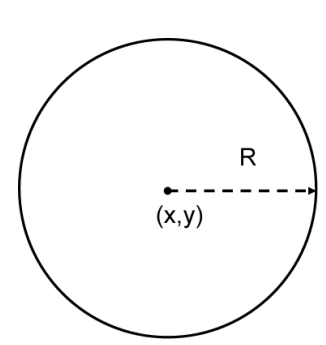

(a)

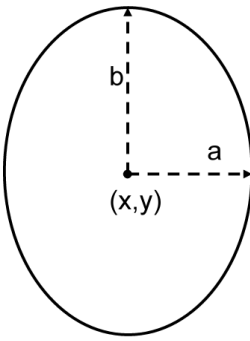

(b)

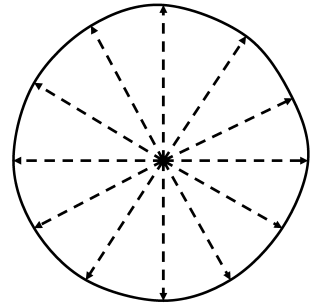

(c)

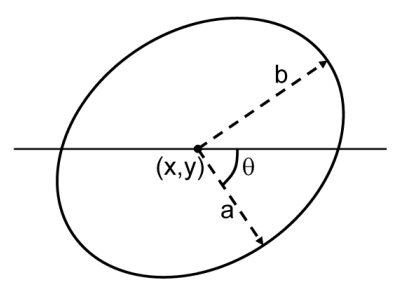

(d)

Figure 5.16: Curve fitting: (a) the circle model, (b) the unrotated ellipse model, (c) the control point based model, $(\mathrm{d})$ the rotated and translated ellipse model.

Therefore correct b-spline-based shape can not be estimated using a partial boundary. Models based on rotated and translated ellipse work well both for frontal and non-frontal iris and require only five parameters to estimate. Mathematically a rotated ellipse is described as:

$$
\begin{gathered}
\frac{\left(\left(x-x_{p 0}\right) \cos \phi+\left(y-y_{p 0}\right) \sin \phi\right)^{2}}{a^{2}}+ \\
\frac{\left(-\left(x-x_{p 0}\right) \sin \phi+\left(y-y_{p 0}\right) \cos \phi\right)^{2}}{b^{2}}=1,
\end{gathered}
$$

where $\left(x_{p 0}, y_{p 0}\right)$ is the center of the pupil and $\phi$ is the angle of rotation. If the pupil boundary is distorted by specular reflections, then a partial boundary is selected using only those points which lie outside the specular reflection mask generated during the inpainting procedure. Because of occlusions caused by the eyelids, top or/and bottom parts of boundary should be avoided. The summary of the conditions for selection of the partial boundary are as follows:

- The part should not be located inside of specular reflection mask. 
- The part should have a clear edge (high gradient value).

- It should be continuous (not crossed by eyelashes).

- It should be verified that the edge is a pupil edge and not an occlusion related edge.

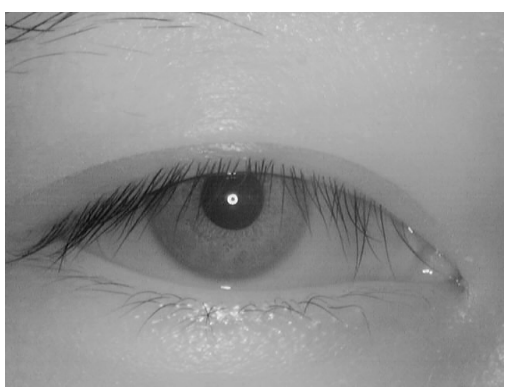

(a)

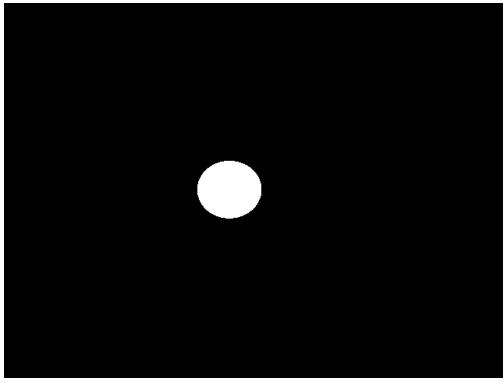

(d)

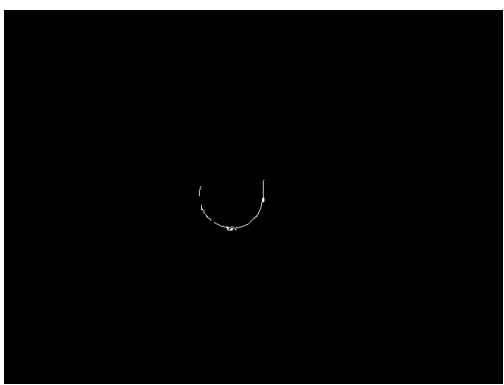

$(\mathrm{g})$

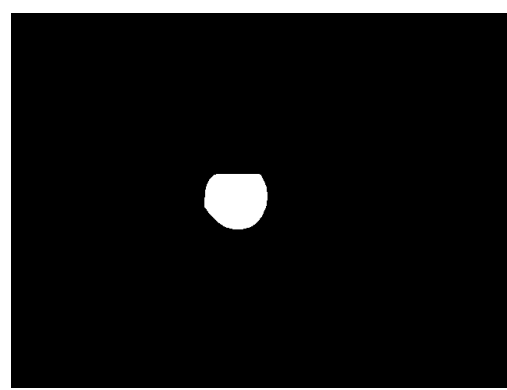

(b)

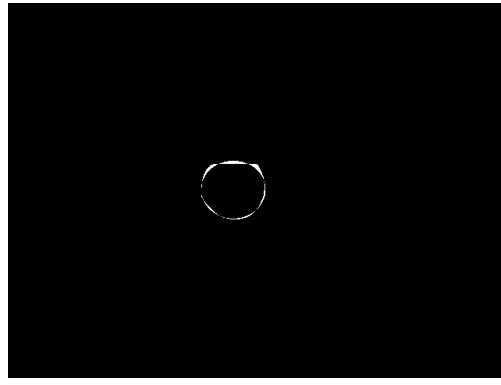

(e)

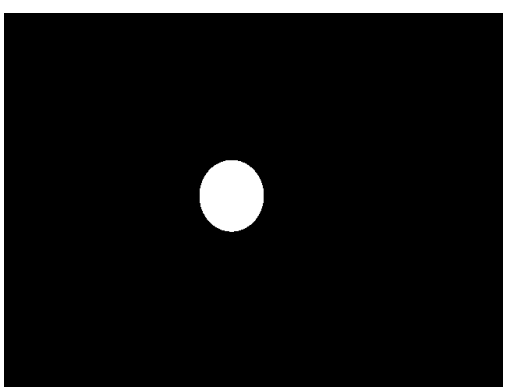

(h)

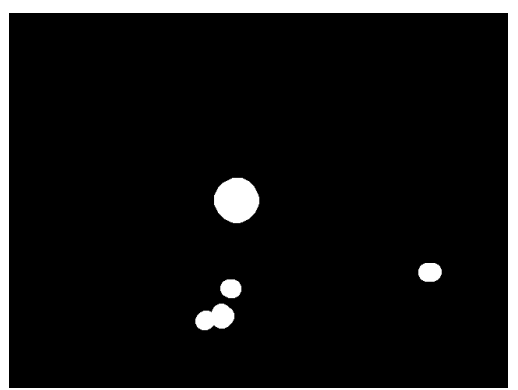

(c)

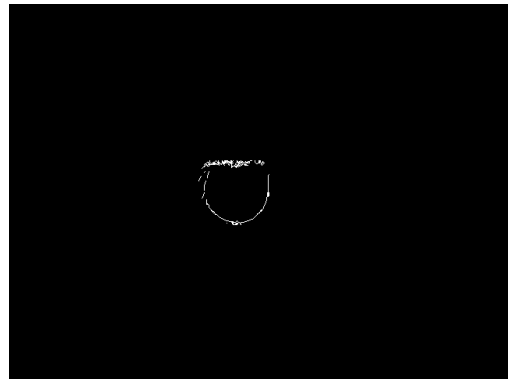

(f)

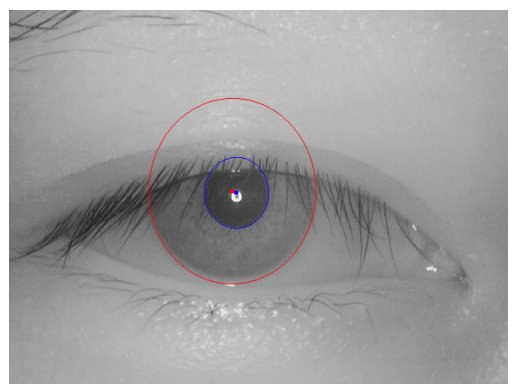

(i)

Figure 5.17: Ellipse fitting: (a) the original image, (b) refined pupil mask, (c) specular reflection mask, (d) ellipse fitting result taking into account specular reflections, (e) the error (the XOR between ellipse fitting with the original refined pupil mask), (f) the edges detected near the refined pupil boundary, (g) selected partial boundary using 4 conditions listed above, (h) the new ellipse fitting result using selected partial boundary, (i) the final segmentation result. 


\section{Evaluation of the Pupil Segmentation}

The goodness of the pupil boundary estimation is verified using two criteria:

- The error between the refined pupil mask and the ellipse fitted into the boundary should be small.

- If only a small part of the pupil remains unoccluded, sections of the pupil boundary have to be well separated.

If the evaluation returns a low score value, then the algorithm uses a blind circle fitting procedure. The initial pupil segmentation result is used to limit the search space by providing an approximate center of the pupil and approximate radius of the circle. This approach works well on frontal view iris images with heavily distorted pupil area.

\subsubsection{Iris Segmentation}

We assume that the shapes of the iris and the pupil are similar and apply the equation (5.2) to segment the iris region. Other assumptions that reduce computational cost can be made. They include the assumption of the same angle $\phi$ and the same ratio of the ellipse parameters $a$ and $b$. These assumptions are especially useful when the iris is heavily occluded or it is strongly rotated.

\section{Image Translation and Inpainting}

To ensure that the search of the limbic boundary is performed over a broad range of parameters describing the ellipse, the image of an iris is translated such that the center of the pupil is aligned with the center of the image. The blank area formed due to the image shift is inpanted to avoid introduction of new edges. An example of an image containing a partial iris is shown in Fig. 5.18. 


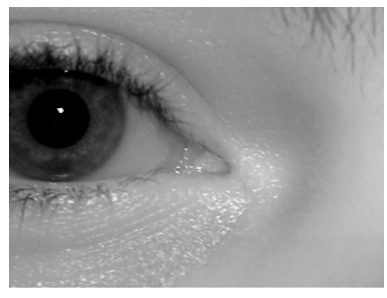

(a)

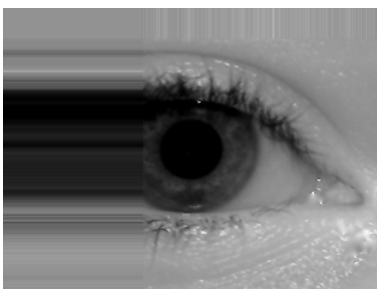

(b)

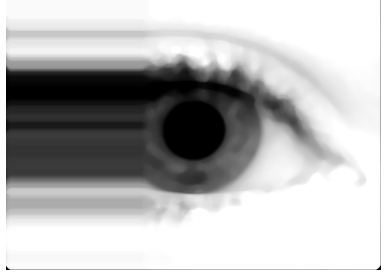

(c)

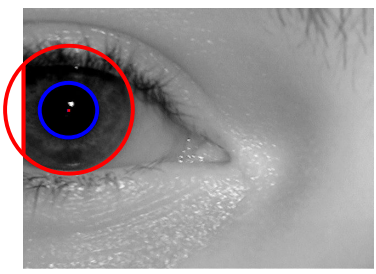

(d)

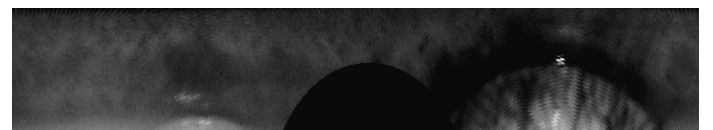

(e)

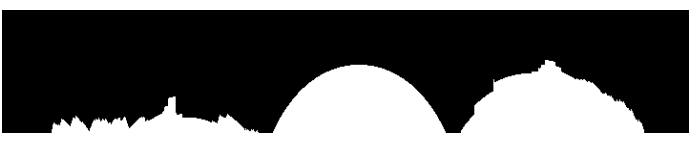

(f)

Figure 5.18: Image translation procedure: (a) the original image, (b) new image after translation and impainting, (c) enhanced image, (d) segmented iris, (e) unwrapped template, (f) unwrapped template mask.

\section{Directional Edge Detector}

We use a method similar to the Daugman's edge detector based on integro-differential operator [46]. Define a space of rotated ellipses as:

$\left\{\left(r, \phi, x_{i 0}, y_{i 0}\right): \frac{\left(\left(x-x_{i 0}\right) \cos \phi+\left(y-y_{i 0}\right) \sin \phi\right)^{2}}{(a r)^{2}}+\frac{\left(-\left(x-x_{i 0}\right) \sin \phi+\left(y-y_{i 0}\right) \cos \phi\right)^{2}}{(b r)^{2}}=1\right\}$,

where the center of the iris $\left(x_{i 0}, y_{i 0}\right)$ is limited to the neighborhood of the center of the pupil $\left(x_{p 0}, y_{p 0}\right), a$ and $b$ are the parameters of the ellipse fitted into the pupil, the scale factor $r$ is limited to be in the range $[1.2,3.5]$, and the parameter $\phi$ is set to be the angle of rotation of the pupil.

Then the problem of finding the rotated ellipse providing the best fit is reduced to the following optimization problem:

$$
\max _{r,\left(x_{i 0}, y_{i 0}\right)}\left[\frac{1}{L\left(r, x_{i 0}, y_{i 0}\right)} \oint_{B\left(r, x_{i 0}, y_{i 0}\right)} F(\nabla(I(x, y))) d s\right],
$$

where $B\left(r, x_{i 0}, y_{i 0}\right)$ is the partial boundary of the ellipse $(5.3), L\left(r, x_{i 0}, y_{i 0}\right)$ is the length of $B\left(r, x_{i 0}, y_{i 0}\right)$, and $F(\nabla(I(x, y)))$ is a function of the image gradient $\nabla(I(x, y))$ detailed below. The function $L\left(r, x_{i 0}, y_{i 0}\right)$ is the normalization given by:

$$
L\left(r, x_{i 0}, y_{i 0}\right)=\oint_{B\left(r, x_{i 0}, y_{i 0}\right)} 1 d s .
$$


Function $F(\nabla(I(x, y)))$ returns adjusted gradient values in the normal direction to the iris boundary between the sclera and the iris region. Use of this function results in reduction of many spurious edges introduced by eyelids, eyelashes, and iris patterns while at the same time retaining those edges caused by the true iris boundary.

The function $F(\nabla(I(x, y)))$ can be evaluated on an unwrapped image as shown in Fig. 5.20. The transformation of an elliptical coordinate system into a circular system is:

$$
\left\{\begin{array}{l}
\left(x-x_{i 0}\right) \cos \phi+\left(y-y_{i 0}\right) \sin \phi=a r \cos \theta^{\prime} \\
-\left(x-x_{i 0}\right) \sin \phi+\left(y-y_{i 0}\right) \cos \phi=b r \sin \theta^{\prime},
\end{array}\right.
$$

where $\theta^{\prime}=\left(\theta-\phi^{\prime}\right)$ and $\phi^{\prime}=\arctan \left(\frac{a}{b} \tan \phi\right)$. The angle $\phi^{\prime}$ is used to map the three o'clock point on the ellipse in the three o'clock point on the circle assuming $\theta=0$. Fig. 5.19 is an illustration of this procedure. Then the normal direction to the ellipse described by equation

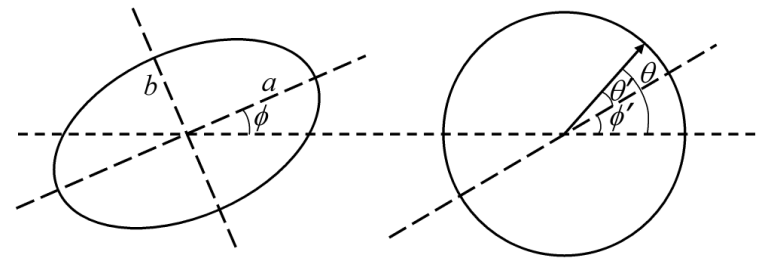

Figure 5.19: A rotated ellipse (left) and its representation in a circular coordinate system.

(5.3) will approximately correspond to the vertical direction in the unwrapped image denoted as $U$. The function $F(\nabla(I(x, y)))$ is then evaluated on the unwrapped image $U$ displayed in the polar coordinate system $(r, \theta)$ :

$$
F\left(\left[U_{\theta}, U_{r}\right]\right)= \begin{cases}U_{r} & U_{r}>0,\left|U_{r}\right| \gg\left|U_{\theta}\right|, \\ 0 & \text { elsewhere }\end{cases}
$$

where $U_{\theta}$ and $U_{r}$ are the gradients of image $U$ in $\theta$ and $r$ directions, respectively.

The polar representation of the iris is further used to efficiently find the boundary of the iris $B\left(r, x_{i 0}, y_{i 0}\right)$. We do not search for a complete boundary that excludes eyelids, eyelashes and other occlusions. We are interested in finding the regions of the iris containing the boundary between iris and sclera only. Once detected, this boundary determines the parameters of the ellipse to be fitted into the iris boundary. Consider the following example. The iris image in Fig. 5.20 is partitioned into 5 regions. The regions E1, E3, and E5 contain 
the boundary between iris and sclera. The other two regions contain boundaries between iris and eyelids and eyelashes. The corresponding unwrapped iris is shown on the right panel in Fig. 5.20. It is easy to see that the parameters of the ellipse to be fitted in the iris can be found by detecting the edges in the regions E1, E3, and E5.

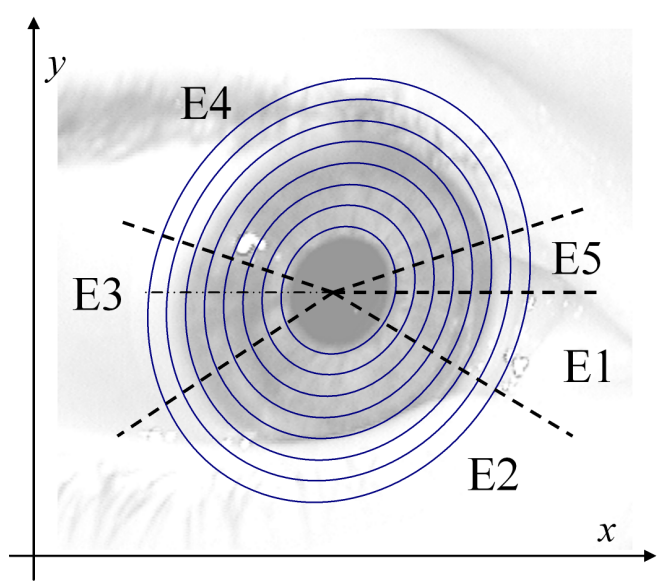

(a)

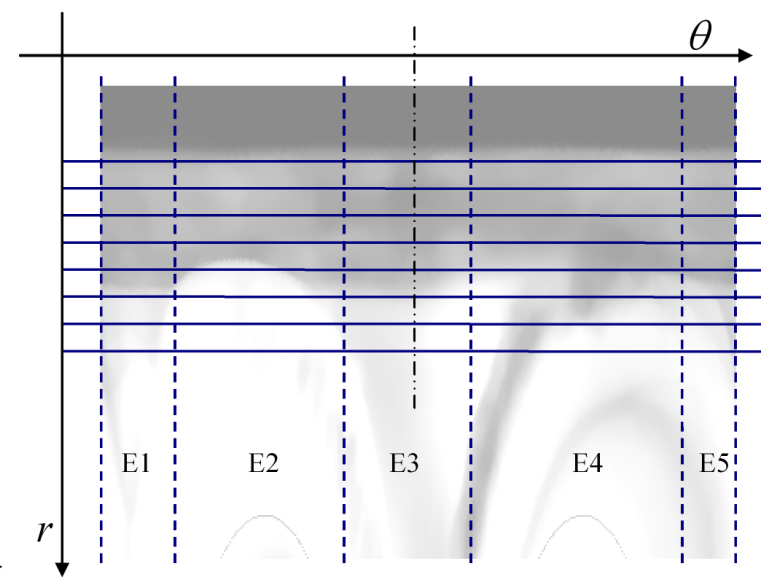

(b)

Figure 5.20: (a) The original image showing an off-angle iris and (b) the same iris transformed into the circular coordinate system and unwrapped. The regions E1, E3, and E5 contain the true boundary between the iris and sclera and are used to find the parameters of the ellipse to be fitted into the iris.

\section{The Off Center Penalty}

Although the center of the pupil and the center of the iris are known to be non-coaxial, we assume that they are located close to one another. To compensate for this effect we involve an off center penalty term $\xi\left(x_{i 0}, y_{i 0}\right)$ defined as

$$
\xi\left(x_{i 0}, y_{i 0}\right)=\frac{1}{1+\frac{\min (a, b)}{\max (a, b)} \frac{\left(x_{i 0}-x_{p 0}\right)^{2}+\left(y_{i 0}-y_{p 0}\right)^{2}}{d_{\max }^{2}}},
$$

where the maximum off center distance $d_{\max }$ is a predetermined quantity. In our experiment we set it to $0.4 \times \max (a, b)$. The penalty term involves ellipse parameters $a$ and $b$, since the distance between pupil center and iris center increases as the off-angle value increases. After 
substituting $\xi\left(x_{i 0}, y_{i 0}\right)$ into (5.4), the equation (5.4) becomes:

$$
\max _{r,\left(x_{i 0}, y_{i 0}\right)}\left[\xi\left(x_{i 0}, y_{i 0}\right) \frac{1}{L\left(r, x_{i 0}, y_{i 0}\right)} \oint_{B\left(r, x_{i 0}, y_{i 0}\right)} F(\nabla(I(x, y))) d s\right] .
$$

Fig. 5.21 demonstrates the relationship between the off center distance and the off angle value using an error bar plot. We used the WVU Off-angle dataset. Note that off center penalty is smaller for off-angle cases compared with frontal view cases because of the scale factor $\frac{\min (a, b)}{\max (a, b)}$.

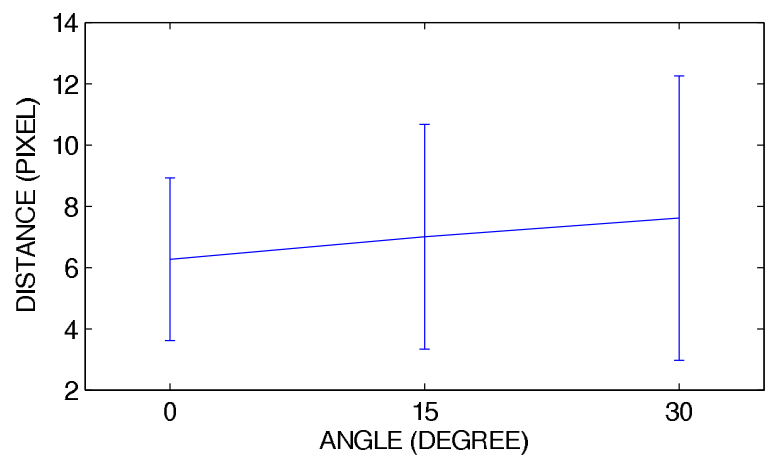

Figure 5.21: The relationship between the off center distance in pixels and the off angle in degrees.

\section{Contrast Compensation}

Besides heavy occlusion, the uneven illumination of the iris may cause an "offset" during the estimation of the iris boundary, since all points on the boundary are treated equally. An example of the iris is shown in Fig. 5.22, where the gradient difference along the iris boundary, resulting from an uneven illumination, caused the left edge of the iris to be more pronounced compared to the right edge. This problem can be easily solved by normalizing the gradients before fitting an ellipse. Note that at this stage, the pupil is segmented. Therefore, the iris images can be unwrapped with respect to the pupil center. The right and left portions of the unwrapped iris are then considered separately. The iris boundaries are detected separately too. The summation of the gradient values along the boundary on each side is used to calculate the weights to balance the segmentation. To achieve the correct 
segmentation of the iris we scale the right and left boundaries of the iris by estimated weights that balance the contribution of the boundaries into the complete ellipse fitted into the iris. The details of the contrast balancing procedure are illustrated in Fig. 5.23.

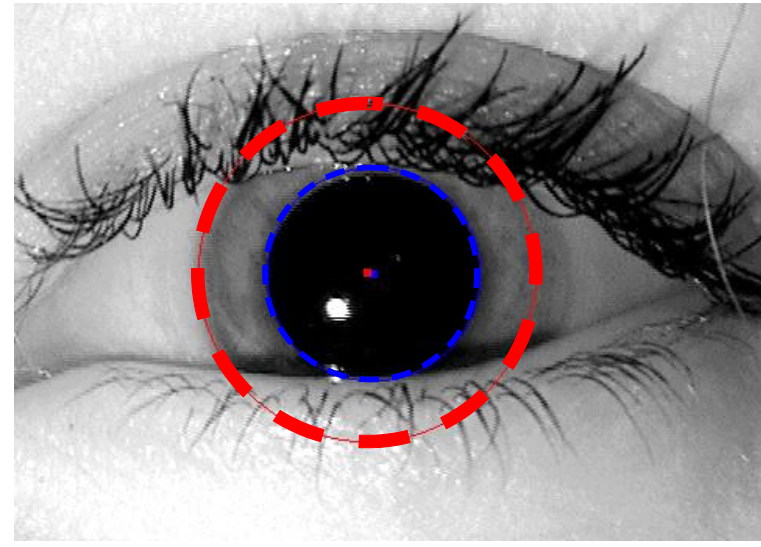

(a)

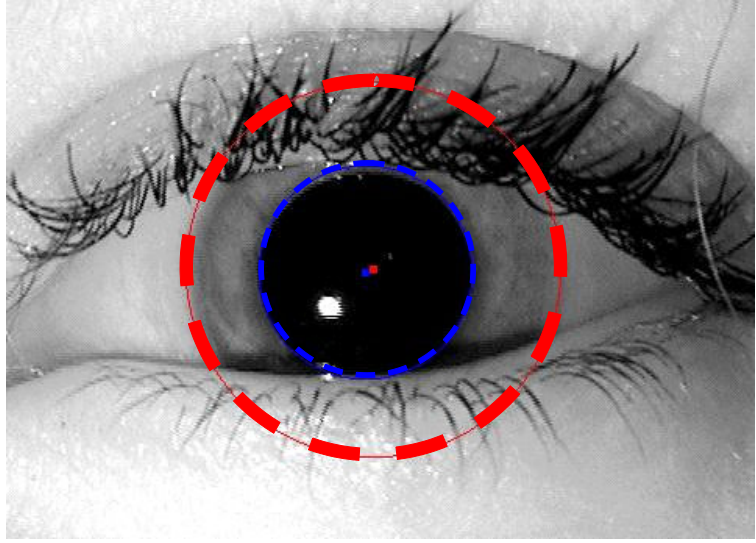

(b)

Figure 5.22: The result of segmentation (a) before contrast balancing and (b) after application of contrast balancing.

\subsubsection{Occlusion Estimation}

The variability of the eyelashes, not only in terms of their position, but also in terms of the direction and intensity, makes occlusion estimation difficult. In this work we implement an occlusion estimation method based on edge detection. This method may not work well on heavily blurred eyelashes and eyelids. For eyelashes, it also can not ensure that all eyelashes are included in the occlusion mask.

Prior to estimating occlusions we apply an adaptive illumination compensation technique. Most of unclear edges due to eyelids are caused by an uneven illumination. An illumination compensation procedure results in brightening shadowed areas and increasing the overall contrast along edges [101]. We involve a Quotient Image-based method [102], [103] to achieve invariance to illumination.

The process of estimating occlusions is further illustrated in Fig. 5.24. The main steps include: detection of horizontal edges (Fig. 5.24-b), smearing of detected edges (Fig. 5.24-c), selection of the iris area based on the earlier obtained segmentation result (Fig. 5.24-d and 


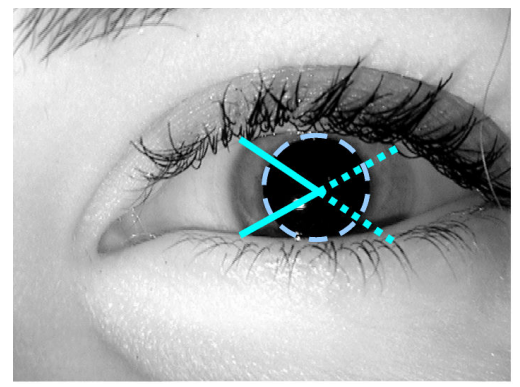

(a)

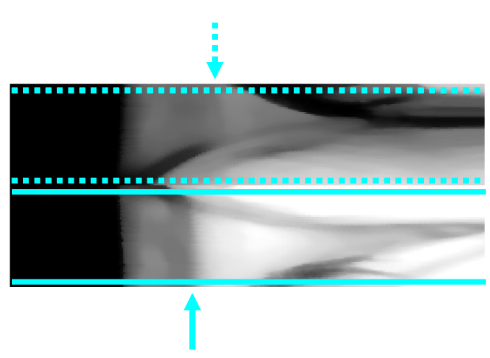

(c)

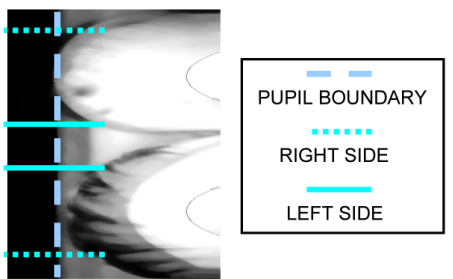

(b)

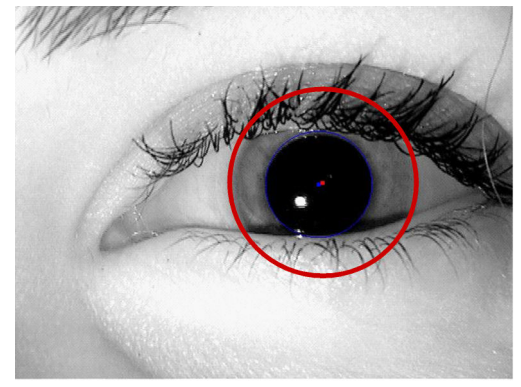

(d)

Figure 5.23: Contrast balancing procedure: (a) the original image with results of pupil segmentation imposed onto the image, (b) the unwrapped image, (c) the iris boundaries are detected separately for left and right portions of the iris, (d) the result of the final iris detection with different contrast compensation weights added on the both sides.

5.24-e) and refinement of the estimated mask based on the connectivity information (Fig. $5.24-\mathrm{f})$.

To detect horizontal edges, we implement a few steps. We first convolve a horizontal Sobel mask with the iris image. Let $b_{y}$ be the result of the convolution. We further compare $b_{y}{ }^{2}$ with the square of the sensitivity threshold $\lambda$ at every pixel location $\left(n_{1}, n_{2}\right)$. If $b_{y}\left(n_{1}, n_{2}\right)^{2}>\lambda^{2}$ and if it is a local maximum, then a horizontal edge point is detected at position $\left(n_{1}, n_{2}\right)$. The parameter $\lambda$ has to be adjusted for different datasets. The smaller the value of $\lambda$, the higher sensitivity of the algorithm to low contrast edges.

Fig. 5.25 illustrates two cases. In the first case, the occlusion mask is estimated without application of illumination compensation technique. The second case presents the results of occlusion estimation after illumination invariance is achieved. 


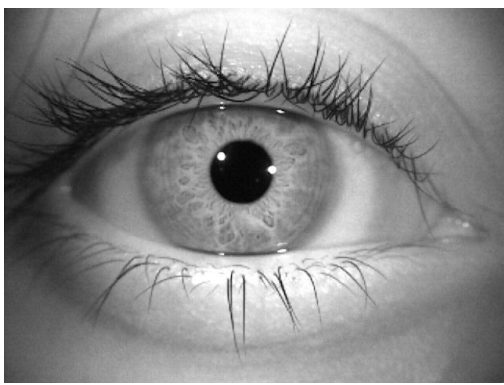

(a)

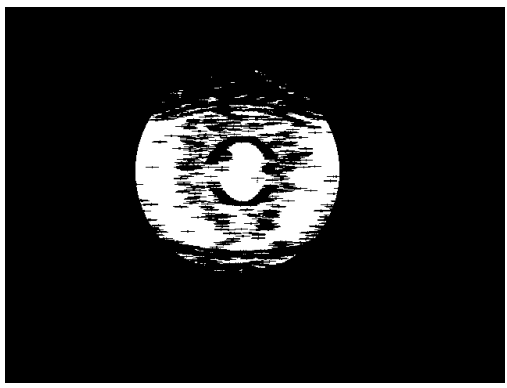

(d)

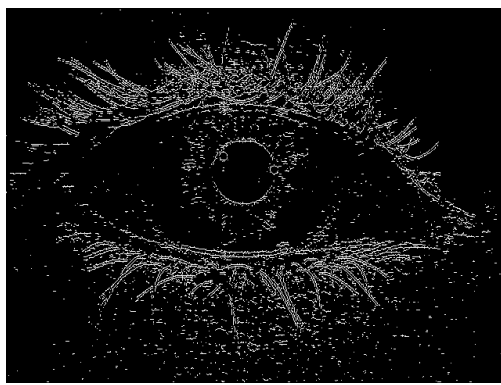

(b)

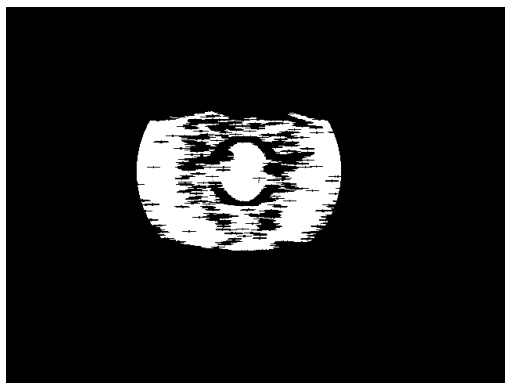

(e)

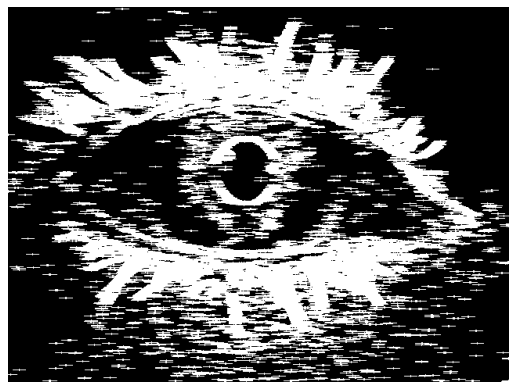

(c)

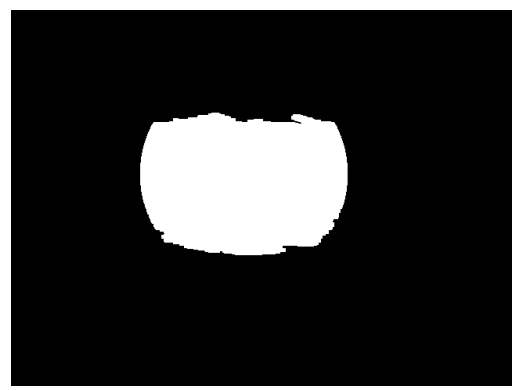

(f)

Figure 5.24: The evolution of the occlusion mask: (a) the original image, (b) the result of the initial edge detection, (c) the edge information after smearing, (d) the result of combination of the mask and the outcome of iris segmentation, (e) the area selected based on connectivity information, and (f) the final result.

\subsubsection{Unwrapping}

To unwrap the iris, we involve a technique similar to Camus and Wildes's technique [79]. Both iris and pupil are described by ellipses. The boundaries are sampled and mapped into circles as described in Section 5.3.3. The ellipse describing the pupil boundary is given by

$$
\left\{\begin{aligned}
x_{p}(\theta)= & a_{p} \cos \left(\theta-\phi_{p}^{\prime}\right) \cos \phi_{p} \\
& -b_{p} \sin \left(\theta-\phi_{p}^{\prime}\right) \sin \phi_{p}+x_{p 0} \\
y_{p}(\theta)= & a_{p} \cos \left(\theta-\phi_{p}^{\prime}\right) \sin \phi_{p} \\
& +b_{p} \sin \left(\theta-\phi_{p}^{\prime}\right) \cos \phi_{p}+y_{p 0}
\end{aligned}\right.
$$




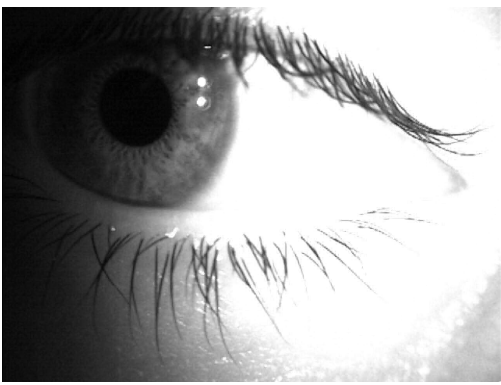

(a)

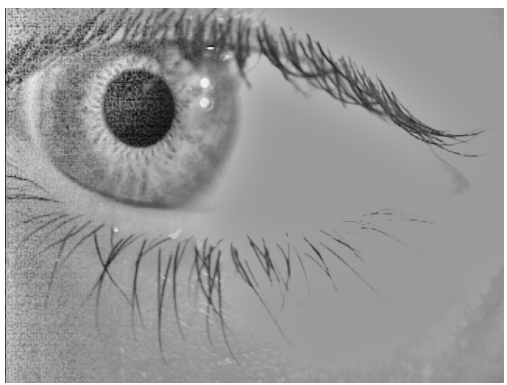

(d)

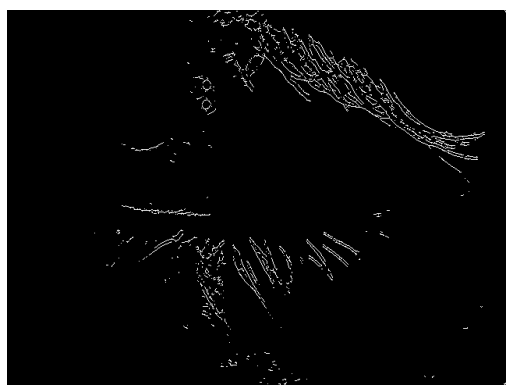

(b)

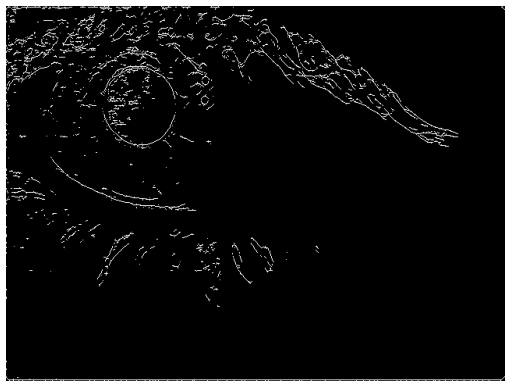

(e)

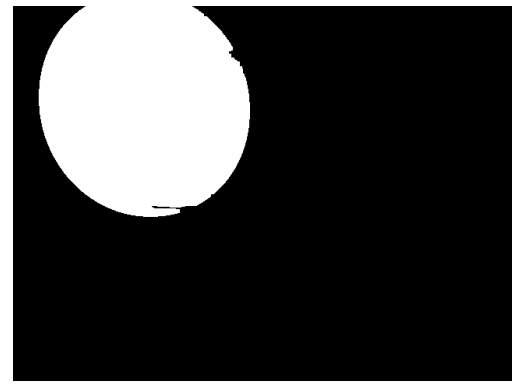

(c)

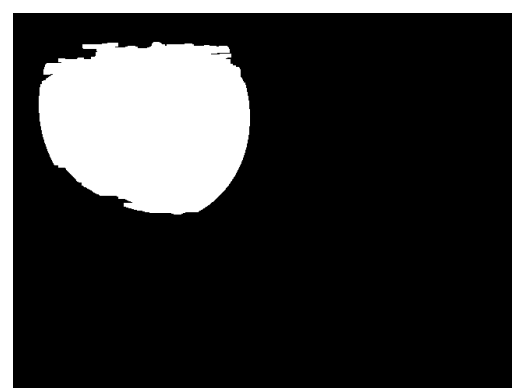

(f)

Figure 5.25: The evolution of the occlusion mask: (a) the original image, (b) the result of the initial edge detection, (c) occlusion mask obtained without illumination preprocessing, (d) image after illumination compensation, (e) the result of horizontal edge detector, (f) occlusion mask obtained after application of illumination compensation.

where $\left(x_{p 0}, y_{p 0}, a_{p}, b_{p}, \phi_{p}\right)$ are the parameters of the ellipse. The iris boundary is described by the ellipse with the parameters $\left\{x_{i 0}, y_{i 0}, a_{i}, b_{i}, \phi_{i}\right\}$ :

$$
\left\{\begin{array}{rl}
x_{i}(\theta)= & a_{i} \cos \left(\theta-\phi_{i}^{\prime}\right) \cos \phi_{i} \\
& -b_{i} \sin \left(\theta-\phi_{i}^{\prime}\right) \sin \phi_{i}+x_{i 0} \\
y_{i}(\theta)= & a_{i} \cos \left(\theta-\phi_{i}^{\prime}\right) \sin \phi_{i} \\
& +b_{i} \sin \left(\theta-\phi_{i}^{\prime}\right) \cos \phi_{i}+y_{i 0}
\end{array} .\right.
$$

The mapping of the point $\left(x^{\prime}, y^{\prime}\right)$ into $(\theta, r)$-point in the pseudo-polar coordinate system is described by:

$$
\left\{\begin{array}{l}
x^{\prime}(\theta, r)=(1-r) x_{p}(\theta)+r x_{i}(\theta) \\
y^{\prime}(\theta, r)=(1-r) y_{p}(\theta)+r y_{i}(\theta)
\end{array},\right.
$$

where $0<r \leq 1,\left(x_{p}, y_{p}\right)$ is a sample point on the pupil boundary, and $\left(x_{i}, y_{i}\right)$ is a sample point on the iris boundary. Finally, the pixel intensity is interpolated using a cubic interpolation method to generate the unwrapped iris. 


\subsection{Performance Evaluation}

The segmentation algorithm is applied to near-IR imagery from a number of public databases. The performance of the algorithm is compared with the performance of two other segmentation algorithms.

\subsubsection{Databases and Parameters}

To analyze the robustness of the proposed segmentation algorithm we evaluate four datasets: CASIA III INT (INT stands for interval), ICE, WVU, and WVU Off-Angle.

CASIA III includes three subsets which are labeled as Interval, Lamp and Twins. It contains 22,051 iris images from more than 700 subjects. The number of images per class varies. As 8 bit gray-level Joint Photographic Experts Group (JPEG) format is used, some compression artifacts in the images can be observed, especially for Interval subset. The multiple specular reflections located inside of the pupil area is another characteristic feature of Interval subset. The resolution of Interval subset images is $320 \times 280$. The resolution of all other images is $640 \times 480$. More information can be found from their website.

ICE dataset includes total 2953 images from 132 individuals. The number of images per class varies. The images were saved using $640 \times 480$ resolution gray-scale Tagged Image File Format (TIFF).

The WVU data consists of 2453 images, representing 359 different classes captured by an OKI-IrisPass hand-held device at a resolution of $640 \times 480$. The number of acquisitions per eye ranged from 2 to 17.

The WVU Off-Angle (WVU-OA) data consists of 560 images, representing 140 different classes captured at a resolution of $720 \times 576$. Each class has 2 frontal images, 1 image at $15^{\circ}$, and 1 image at $30^{\circ}$.

Iris databases used in our experiments have distinct resolution, optical characteristics, and noise. Based on our observation, iris images from ICE dataset have relatively uniform intensity values in the area of pupil excluding the regions of specular reflections. The boundaries between the regions of specular reflection and the pupil are relatively clear except a few cases of heavily blurred images. Iris images from WVU dataset have a larger variance 
of the pixel intensities within the pupil area and a large number of images characterized by low contrast. Iris images from WVU-OA dataset are generally of good quality. CASIA III "interval" directory is characterized by complex specular reflections. Based on analyzed characteristics of images from different datasets, we identified a number of parameters of the segmentation algorithm that need to be adjusted for improved segmentation. The main parameters include:

- Maximum pupil intensity value $\tau$ (introduced in section 5.3.1). This is the maximum value of pupil intensity around the area affected by specular reflections.

- A threshold $\gamma_{1}$ (introduced in section 5.3.1). This is a threshold used to detect regions of specular reflections within the area of pupil.

- A threshold $\gamma_{2}$ (introduced in section 5.3.1). This is a threshold used to detect specularities in the area outside the pupil.

- A parameter $\lambda$ used to modify sensitivity of the horizontal edge detector (introduced in section 5.3.4). This parameter is used in the process of occlusion estimation. The parameter is adjusted based on the average image contrast of a dataset.

Table 5.1 summarizes the estimated parameters $\tau, \gamma_{1}, \gamma_{2}$, and $\lambda$ for the imagery from four databases.

Table 5.1: Parameter settings for different datasets

\begin{tabular}{|c|c|c|c|c|}
\hline & $\tau$ & $\gamma_{1}$ & $\gamma_{2}$ & $\lambda$ \\
\hline CASIA III INT & 100 & 100 & 240 & 4 \\
\hline ICE & 50 & 50 & 240 & 4 \\
\hline WVU & 50 & 50 & 240 & 4 \\
\hline WVU-OA & 50 & 50 & 240 & 2.3 \\
\hline
\end{tabular}

\subsubsection{Visual Evaluation}

Since the ground truth is not available, we appeal to a subjective (visual) evaluation as shown in Fig. 5.26. We select two criteria as a measure of visual evaluation: the maximum 
offset of the pupil boundary, $\epsilon_{p}$, and the maximum offset of the iris boundary $\epsilon_{i}$. The two offsets will be compared with the minimum distance between the iris boundary and pupil, $\min _{d}$. All segmentation results will be placed in four categories:

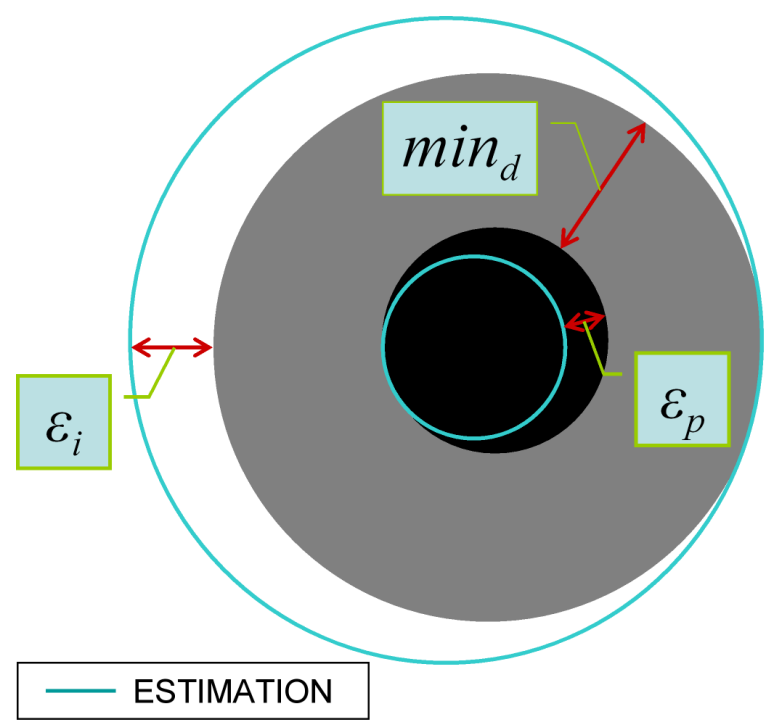

Figure 5.26: Criteria used for visual evaluation of the proposed segmentation algorithm.

Good: $\epsilon_{p} / \min _{d}<5 \%$ and $\epsilon_{i} / \min _{d}<10 \%$. There is no clear boundary offset between segmentation curves and true iris or pupil boundary. The error of the occlusion estimation is less than $12.5 \%$ of unmasked area.

Fair: $\left(5 \% \leq \epsilon_{p} / \min _{d}<10 \%\right.$ and $\epsilon_{i} /$ min $\left._{d}<20 \%\right)$ or $\left(\epsilon_{p} / \min _{d}<10 \%\right.$ and $10 \% \leq$ $\left.\epsilon_{i} / \min _{d}<20 \%\right)$. The error of the occlusion estimation is less than $25 \%$ of unmasked area.

Poor: $\left(10 \% \leq \epsilon_{p} / \min _{d}<20 \%\right.$ and $\left.\epsilon_{i} / \min _{d}<35 \%\right)$ or $\left(\epsilon_{p} / \min _{d}<20 \%\right.$ and $20 \% \leq$ $\left.\epsilon_{i} / \min _{d}<35 \%\right)$. The error of the occlusion estimation is larger than $25 \%$ of unmasked area.

Bad: Everything else.

Examples of good, fair, poor, and bad segmentation are shown in Fig. 5.27. Fig. 5.28 


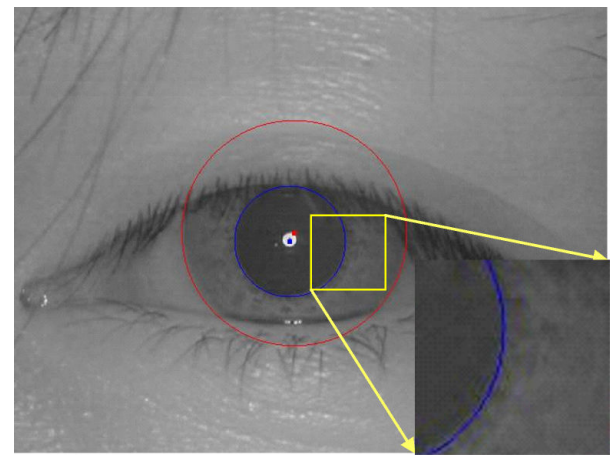

(a)

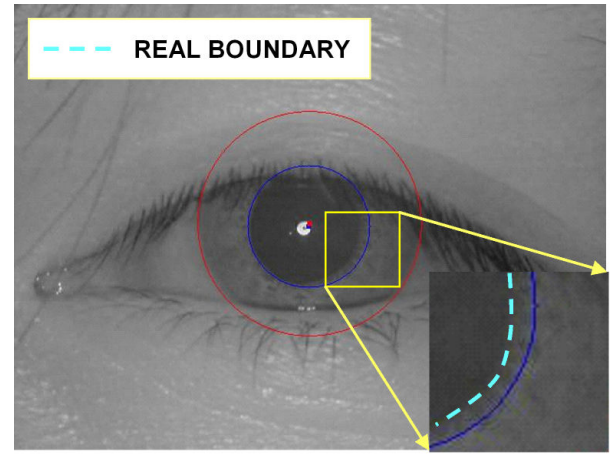

(c)

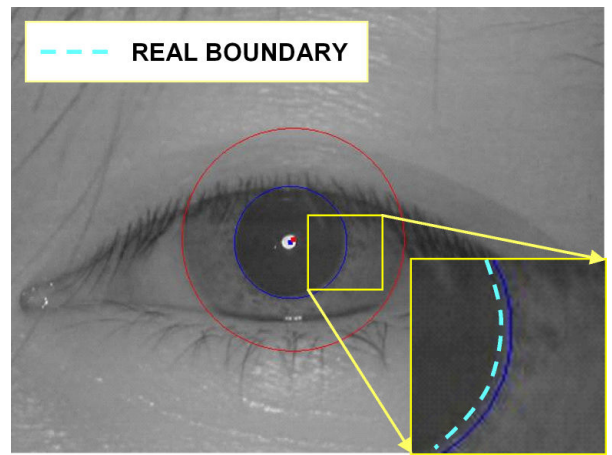

(b)

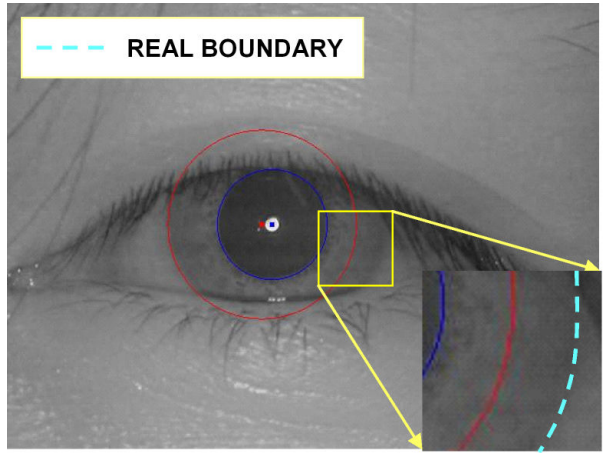

(d)

Figure 5.27: Examples of: (a) good, (b) fair, (c) poor, and (d) bad segmentations.

displays two examples of correctly segmented iris images from WVU and WVU-OA datasets.

We compare our segmentation method against two well established algorithms, Libor Masek's [45], [41] and Camus and Wildes's [79] algorithms. Masek's algorithm is publicly available and its modified $\mathrm{C}++$ version was used as a baseline for the ICE phase I (ICE 2005) competition. We use our own implementation of Camus and Wildes's algorithm described in [79]. Table 5.2 compares the success rate in segmentation of the three algorithms. Overall, the proposed algorithm significantly outperforms the other algorithms. Other algorithms have some natural limitations, such as circle fitting for Masek's implementation which does not perform well on off-angle images. However, a few cases can be found when the algorithms used for performance comparison outperform our algorithm. Fig. 5.29 presents an example, where a simple edge-based circle fitting produces better results than our algorithm. This is an example of heavily occluded iris region. 

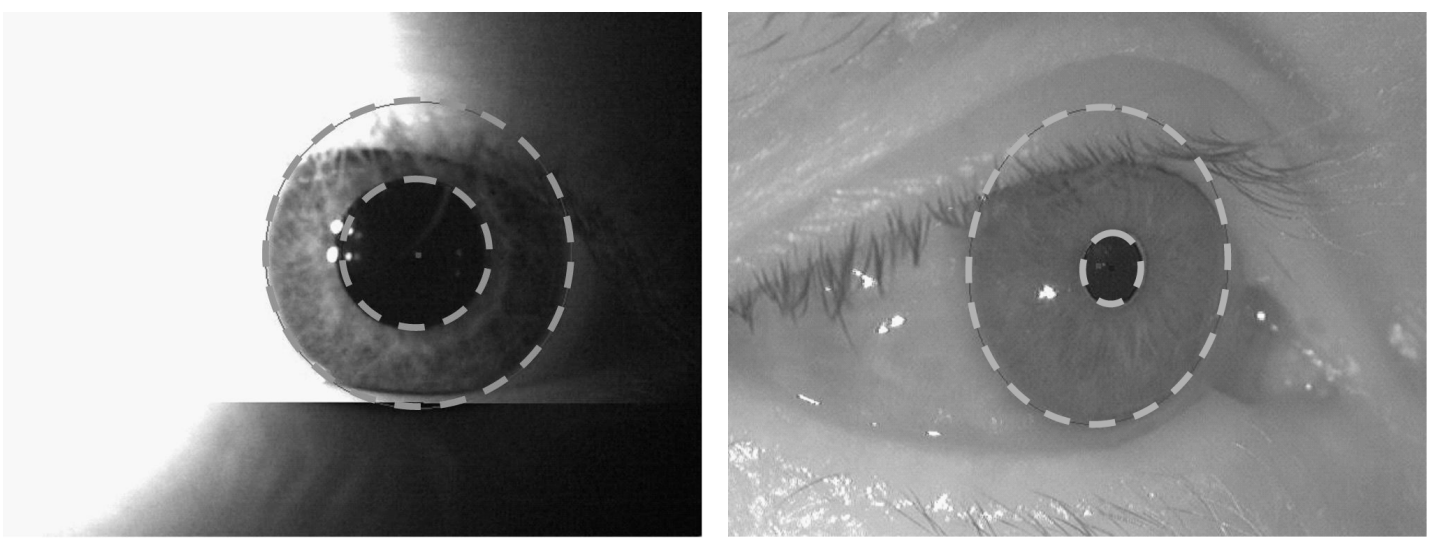

Figure 5.28: Examples of correctly segmented iris images from WVU and WVU-OA datasets.

Table 5.2: Success rate in segmentation

\begin{tabular}{|c|c|c|c|}
\hline & Masek & Camus and Wildes & Proposed \\
\hline CASIA III INT & $88.89 \%$ & $92.39 \%$ & $99.06 \%$ \\
\hline ICE & $91.20 \%$ & $90.79 \%$ & $98.95 \%$ \\
\hline WVU & $64.77 \%$ & $85.24 \%$ & $97.92 \%$ \\
\hline WVU-OA & $71.43 \%$ & $70.00 \%$ & $99.82 \%$ \\
\hline
\end{tabular}

Table 5.3 contains the results of manual evaluation of the segmentation performance of the proposed algorithm. Note that combined "good" and "fair" results provide us with the

Table 5.3: The results of manual performance evaluation

\begin{tabular}{|c|c|c|c|c|c|c|}
\hline Dataset & Correct=Good+Fair & Good & Fair & Poor & Bad & Poor+Bad \\
\hline CASIA III INT & $99.06 \%$ & $96.31 \%$ & $2.75 \%$ & $0.49 \%$ & $0.45 \%$ & $0.94 \%$ \\
\hline ICE & $98.95 \%$ & $91.80 \%$ & $7.15 \%$ & $0.17 \%$ & $0.88 \%$ & $1.05 \%$ \\
\hline WVU & $97.92 \%$ & $90.05 \%$ & $7.87 \%$ & $0.86 \%$ & $1.22 \%$ & $2.08 \%$ \\
\hline WVU-OA & $99.82 \%$ & $93.39 \%$ & $6.43 \%$ & $0.18 \%$ & $0 \%$ & $0.18 \%$ \\
\hline
\end{tabular}

percentage of correctly segmented iris images.

\subsubsection{Recognition Performance}

Fig. 5.30 illustrates ROC performance of our segmentation algorithm compared with the baseline algorithm used in the ICE 2005 competition. Both algorithms use Libor Masek's encoding algorithm. The difference is in segmentation. The baseline algorithm is described 


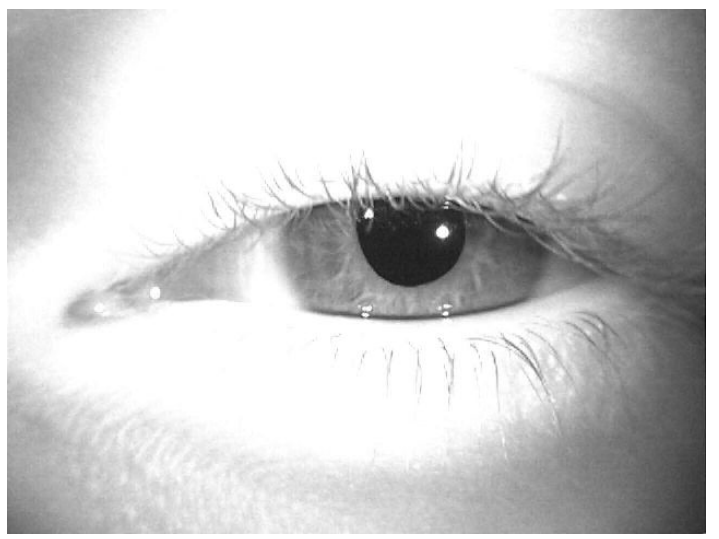

(a)

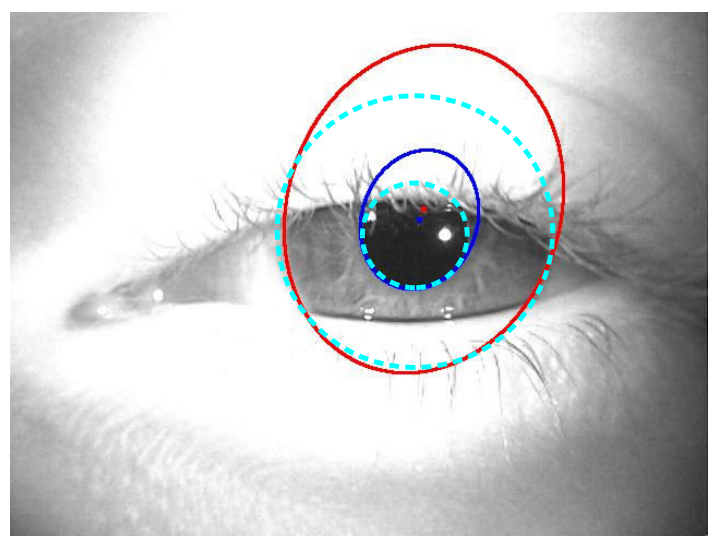

(b)

Figure 5.29: Segmentation of occluded iris images: (a) the original image (from WVU dataset), (b) circular fit due to a simple edge-based approach (dashed line) and elliptical fit due to our algorithm (solid line).

in [45], [41]. The performance of other ICE 2005 participants is not shown, since we do not analyze performance of encoding techniques. For the right eye test, our algorithm achieves $97.95 \%$ verification rate (VR) at $0.1 \%$ false accept rate (FAR), while the baseline algorithms achieves $85.5 \%$. For the left eye test, our algorithm achieves $96.85 \%$ VR at $0.1 \%$ FAR, while the baseline algorithms achieves $85.45 \%$. Note the substantial improvement in recognition performance due to application of our segmentation algorithm adapted to deal with nonideal iris imagery.

The speed of the three algorithms was evaluated using a personal computer equipped with Intel ${ }^{\circledR}$ Pentium ${ }^{\circledR}$ IV $3 \mathrm{GHz}$ processor and $2 \mathrm{~GB}$ of RAM. All segmentation techniques were implemented in Matlab. Implementation of our method relied on functions from Statistics and Image Processing Toolboxes. Optimization of the proposed segmentation method was not a focus of this work. Therefore, the results are provided for non-optimized version of our method. On the contrary, implementation of Camus and Wildes's algorithm relies on multiresolution approach (we used functions from a multiresolution Toolbox in Matlab [104] to reproduce Camus and Wildes's algorithm). The running time of the methods and algorithms is summarized in Table 5.4. Camus and Wildes's algorithm is more efficient when applied to high resolution (large in size) images. The proposed algorithm performs relatively well, especially if we notice that our algorithm is not optimized. 


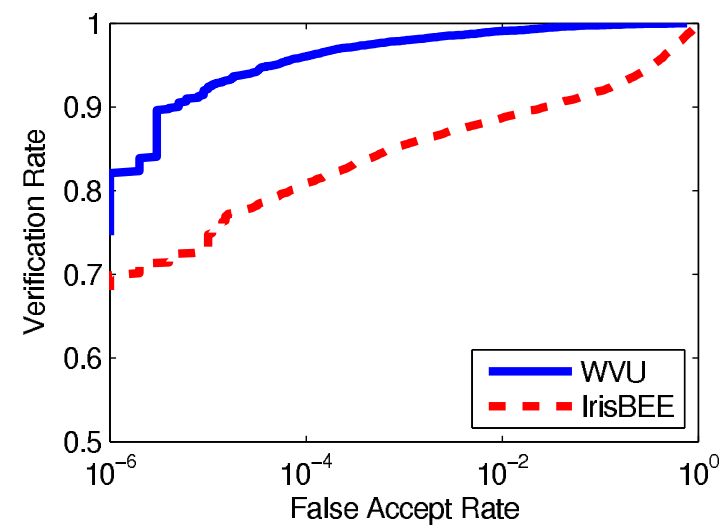

(a)

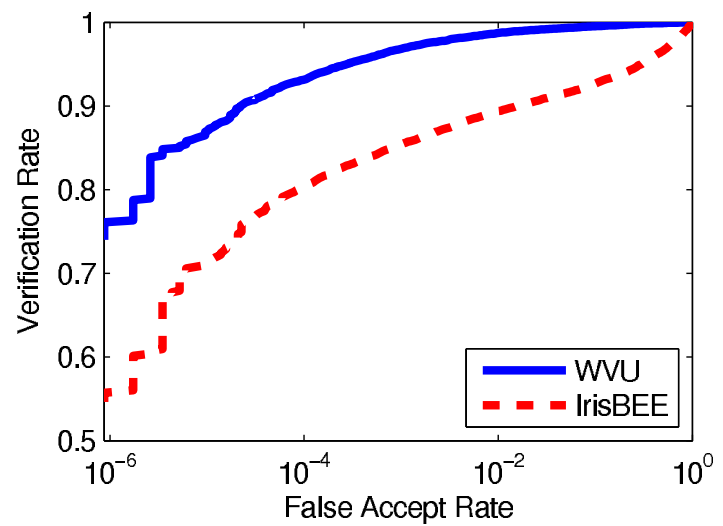

(b)

Figure 5.30: ROC performance of (a) right and (b) left eye for ICE data. IrisBEE is a version of Masek's algorithm. IrisBEE composed of segmentation, encoding, and matching algorithms was used as a baseline algorithm in ICE 2005. The solid ROC curves on the left and right panels in the figure are obtained using the segmentation algorithm proposed in this chapter and encoding and matching algorithms of the IrisBEE.

Table 5.4: Complexity (resolution vs. running time (in seconds))

\begin{tabular}{|c|c|c|c|c|c|}
\hline & Masek & Camus and Wildes & $\begin{array}{c}\text { Proposed } \\
\text { (step size 1) }\end{array}$ & $\begin{array}{c}\text { Proposed } \\
\text { (step size 2) }\end{array}$ & $\begin{array}{c}\text { Proposed } \\
\text { (step size 3) }\end{array}$ \\
\hline $640 \times 480$ & 439 & 69 & 138 & 108 & 101 \\
\hline $480 \times 360$ & 324 & 42 & 85 & 70 & 67 \\
\hline $320 \times 240$ & 89 & 27 & 34 & 30 & 30 \\
\hline $160 \times 120$ & 18 & 10 & 10 & 9 & 9 \\
\hline
\end{tabular}

The proposed non-optimized segmentation procedure may appear to be slow when the algorithm operates on high resolution images. This inefficiency is due to filtering operations and is due to using an exhaustive search to select the best candidate for the iris center. In a database such as ICE 2005 database the off-center distance can reach up to 11 pixels. For the search over a circular area this results in about 380 iris center candidates to consider. The distribution of the off-center distances for ICE 2005 can be found in Fig. 5.31. An example of the image with a large off-center distance is shown in Fig. 5.32. This procedure can be sped up considerably by involving multi-resolution analysis similar to the one adopted by Camus and Wildes.

We made the first attempt to speed up our segmentation procedure by applying a hierarchical search algorithm, where prospective centers are sampled roughly at first, then the 


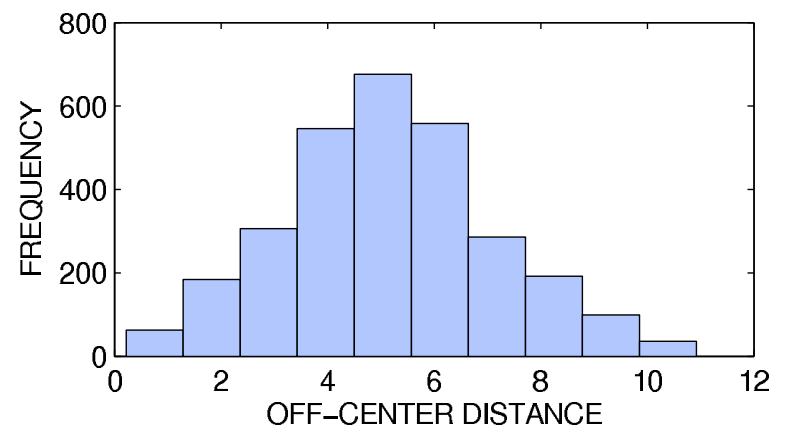

Figure 5.31: The distribution of the off-center distances for ICE dataset.

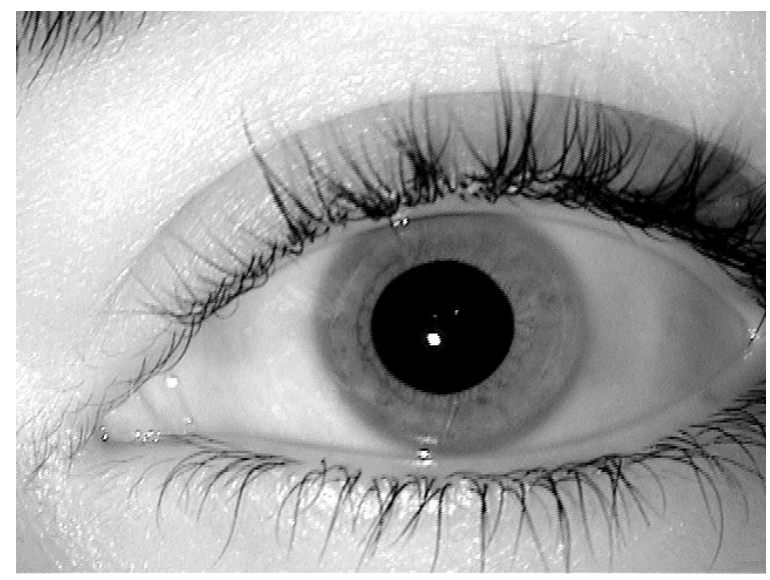

Figure 5.32: An example of large off-center distance image from ICE dataset (243265.tiff).

search is refined. The last three columns in Table 5.4 are the speed of exhaustive approach and optimized approach due to simply changing the search step to two and three, respectively. The reduction of running time due to the initial optimization is clearly observed. Since our segmentation procedure relies on a large number of enhancement and image processing procedures, changing the search step in searching the iris center may not be sufficient. We believe that applying multi-resolution analysis similar to the one adopted by Camus and Wildes will result in a better solution.

\subsubsection{Automatic Algorithm for Evaluating the Precision}

If segmentation is not performed at a certain precision, the error of segmentation will further propagate and will be amplified during the proceeding processing, encoding, and 
matching steps. This emphasizes a critical need in designing robust iris segmentation algorithms and together with it a need of automatic algorithms evaluating the precision (accuracy) of iris segmentation. Automatic algorithm evaluating the precision of segmentation plays important role for two reasons: (1) it can be placed into a feedback loop to enforce another run of segmentation algorithm that may include more sophisticated steps for high precision segmentation and (2) the outcome of this evaluation can be treated as a quality factor and thus can be used to design a quality driven adaptive iris recognition system.

To design an automatic algorithm capable of evaluating the precision of iris segmentation is not an easy task. Moreover, it is a questionable task, since no ground truth for correct iris segmentation is available. While it is almost impossible to design a perfect automatic algorithm for segmentation quality check, we can design an automatic algorithm which rules out iris images with wrong segmentation.

The algorithm that we propose relies on the following tests: (1) The pupil (relative to the image resolution) has to be of sufficient size (in pixels) for it to be well segmented. Therefore, we can use a threshold-based test that checks the size of the pupil. (2) The second test analyzes estimated boundaries of the iris and pupil. If boundaries are well estimated, then the cumulative gradient along the boundary takes a large value. (3) The third test evaluates the relationship among intensities of the pupil, iris, and sclera. Excluding occluded portions of the iris, the average intensity value of the pupil is traditionally smaller then the average intensity value of the iris. The average intensity value of the iris is traditionally smaller than the average intensity value of the sclera.

These tests can be easily performed if the region of segmented iris is expanded beyond its boundaries by $10 \%$ in the direction towards the center of the pupil and by $20 \%$ in the direction of the sclera. Then both the edge information and intensity information can be concluded on by using image regions adjacent to the pupil and iris boundaries.

To evaluate the cumulative intensity gradient along the estimated pupil and iris boundaries, we unwrap the image. First, a band covering the estimated pupil boundary (Fig. 5.33 (d)) is analyzed. For each horizontal pixel in unwrapped image we evaluate the intensity gradient along the vertical direction. If a gradient value for one of vertical pixels exceeds a specified threshold, we will say that at this location (horizontal location) the boundary 
is detected (Fig. 5.33 (e)). We further count the number of horizontal locations with the gradient exceeding the threshold and divide by the total number of horizontal pixels in the unwrapped iris. A similar evaluation is performed for the iris boundary (Fig. 5.33 (f)). Since in majority of cases the limbic boundary is not sufficiently sharp, a vertically compressed version is used in place of the original unwrapped image to detect the edge (Fig. $5.33(\mathrm{~g})$ ). Denote by $\Omega_{p}$ and $\Omega_{i}$ the percentage of horizontal pixels with the gradient exceeding the prespecified threshold where $\Omega_{p}$ is for the pupil boundary and $\Omega_{i}$ is for the limbic boundary. However, occluded parts will not be taken into account. The larger the values of these parameters, the better are the estimates of the boundaries. The procedure is illustrated in Fig. 5.33. The decisions made by the automatic test are summarized below:

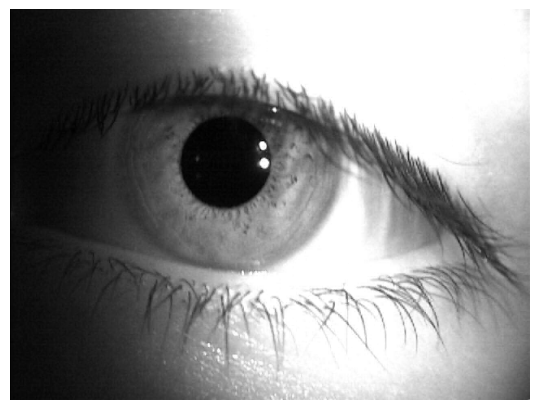

(a)

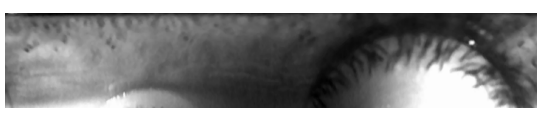

(b)

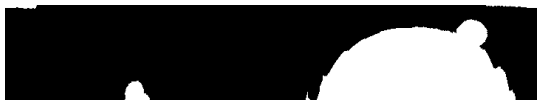

(c)

$(d)$

(e)

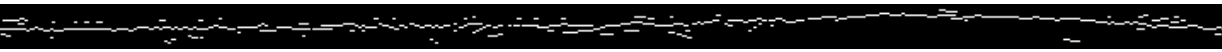

$(f)$

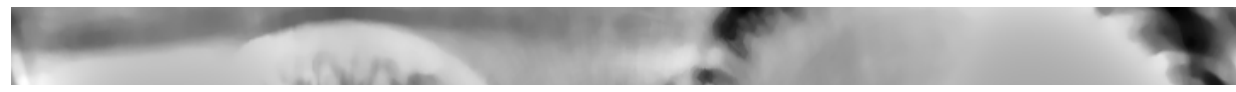

$(g)$

Figure 5.33: The steps performed by the automatic algorithm for evaluation of precision of iris segmentation: (a) the original image, (b) the unwrapped template, (c) the unwrapped mask, (d) the extended portion of unwrapped iris on the pupil side, (e) the result of edge detection on the pupil side, (f) the extended portion of unwrapped iris on the sclera side, (g) the result of edge detection on the sclera side. 
- Correct: $1 \geq \Omega_{p}>0.8$ and $1 \geq \Omega_{i}>0.7$.

- Incorrect: Otherwise.

The two decisions can be further subdivided into four categories, good and fair (resulting in correct segmentation) and poor and bad (resulting into incorrect segmentation), by refining the criteria.

The parameter $\Omega_{i}$ can also be used to evaluate the precision of occlusion estimation. The occlusion estimation is labeled as "wrong" if $\Omega_{i}<0.85$. For the example in Fig. 5.33, $\Omega_{p}=0.9781$ and $\Omega_{i}=0.9883$. In this case, our automatic algorithm concludes that the image in Fig. 5.33 is well segmented and has correctly estimated occlusion mask.

Table 5.5 summarizes the results of application of our automatic algorithm to evaluate the precision of iris segmentation performed by our robust segmentation algorithm. Table

Table 5.5: Summary of decisions made by automatic algorithm

\begin{tabular}{|c|c|c|c|}
\hline Dataset & Correct & Incorrect & Occlusion \\
\hline CASIA III INT & $96.73 \%$ & $3.27 \%$ & $88.63 \%$ \\
\hline ICE & $92.31 \%$ & $7.69 \%$ & $90.25 \%$ \\
\hline WVU & $95.97 \%$ & $4.03 \%$ & $92.38 \%$ \\
\hline WVU-OA & $96.96 \%$ & $3.04 \%$ & $91.43 \%$ \\
\hline
\end{tabular}

5.6 presents the results of the manual evaluation. From Tables 5.5 and 5.6 we may conclude

Table 5.6: Summary of decisions made by manual evaluation

\begin{tabular}{|c|c|c|c|}
\hline Dataset & Correct & Incorrect & Occlusion \\
\hline CASIA III INT & $99.06 \%$ & $0.94 \%$ & $97.74 \%$ \\
\hline ICE & $98.95 \%$ & $1.05 \%$ & $92.79 \%$ \\
\hline WVU & $97.92 \%$ & $2.08 \%$ & $91.97 \%$ \\
\hline WVU-OA & $99.82 \%$ & $0.18 \%$ & $93.75 \%$ \\
\hline
\end{tabular}

that there are only a few images where the automatic and manual evaluations disagree.

Table 5.7 presents a summary of the percentages of false alarms and false positives. The false alarms are percentages of all images that are classified as incorrectly segmented using automatic algorithm and correctly segmented using manual evaluation. The false positives are percentages of all images that are classified as correctly segmented by the automatic 
algorithm and classified as incorrectly segmented through the manual evaluation. Note that the ICE dataset has a relatively large percentage of false positives compared to other databases. This is due to a large number of blurred images. However, the overall performance comparison is very satisfying. A more precise threshold setting for better segmentation will result in a lower false positive rate, but will increase the false alarm rate.

Table 5.7: Automatic vs. manual

\begin{tabular}{|c|c|c|c|}
\hline Dataset & False Alarm & False Positive & $\begin{array}{c}\text { False Alarm } \\
\text { on Occlusion }\end{array}$ \\
\hline CASIA III INT & $2.41 \%$ & $0.23 \%$ & $9.42 \%$ \\
\hline ICE & $6.64 \%$ & $0 \%$ & $5.72 \%$ \\
\hline WVU & $2.69 \%$ & $0.73 \%$ & $3.67 \%$ \\
\hline WVU-OA & $2.86 \%$ & $0 \%$ & $3.39 \%$ \\
\hline
\end{tabular}

With respect to occlusion, false alarm is defined as the percentage of images that are classified through manual evaluation as images with good segmentation and classified by the automatic algorithm as wrong occlusion. The occlusion false alarm is presented in the third column of Table 5.7. The high percentage values on the dataset CASIA III INT are due to iris images with partially low contrast between the iris and sclera.

\subsection{Conclusions and Future Work}

A methodology for robust iris segmentation designed specifically for non-ideal irises has been proposed. This methodology utilizes shape, intensity, and location information for pupil/iris localization. An ellipse based model is used to contour the estimated boundaries for pupil and iris regions which demonstrates robustness to non-frontal iris images. A contrast balancing technique is introduced to reliably detect iris boundary under the condition of uneven illumination. The occlusion mask is carefully estimated using robust edge detector. We have evaluated four distinct datasets, ideal and non-ideal, in order to demonstrate the robustness of our algorithm. We have compared our results to that of Masek and Camus and Wildes. Using all datasets, we achieve an average increase in segmentation performance of $17.1 \%$ over the latter segmentation methodologies. Optimization of the speed of the 
algorithm was beyond the scope of this chapter.

An automatic algorithm evaluating the precision of segmentation is also proposed as a necessary component of the iris segmentation algorithm. Since any automatic iris segmentation algorithm faces challenging images that result in poor or bad segmentation, the purpose of an automatic algorithm evaluating the precision of segmentation is to block these images from sending them for further processing and recognition.

In the future we plan to address a number of problems that we observed while performing this work. We intend to address the issue of the speed of our algorithm. The goal is to make implementation to perform online.

We would also like to explore new methods and processing tools described in the literature on iris segmentation and iris recognition. For example, application of active contours as a tool for a potentially more precise segmentation is a natural extension to our current work. We will use ellipse fitting as an initial guess. 


\section{Chapter 6}

\section{Iris Encoding}

In majority of iris recognition systems, segmentation of an iris image is followed by encoding. Daugman's iris recognition algorithm introduced in early 90s and later undergoing continuous refinements remains potentially the most efficient and scalable in iris field. In this chapter, we present several extensions from the original iris encoding concept.

\subsection{Local Ordinal Binary Extension}

In this section, we design and test an algorithm that can be used both individually and as a natural extension scheme to Gabor wavelet-based algorithm. It is based on the local ordinal information extracted from original unfiltered images. This scheme holds a number of promises: (1) it is robust with respect to a number of nonidealities in iris images and (2) because of the binary nature of the local ordinal information this scheme can be flawlessly integrated into the traditional filter-based recognition systems. The proposed scheme was extensively tested individually and when combined with Gabor wavelet-based approach.

\subsubsection{Introduction}

Among existing iris recognition techniques, Daugman's 2D Gabor wavelet-based encoding algorithm $[8,46,94]$ remains the most popular and efficient technique for a frontal view high quality iris imagery. However, since introduction of new research directions such as iris at a distance and non-ideal iris the capabilities of Daugman's algorithms applied to non-ideal 
iris images were questioned. While most researchers focus on designing novel preprocessing, iris unwrapping, and encoding procedures for non-ideal iris (including Daugman himself), in this work we show that by combining Daugman's approach with ordinal binary coding at the matching score level leads to considerable recognition gain, efficiency and robustness of a traditional Gabor wavelet-based algorithm.

Local Binary Pattern (LBP) technique $[105,106]$, a subcase of ordinal binary encoding, was previously introduced as an iris encoding technique by Sun et al. [26]. While Sun et al. propose to use LBP as an individual encoding technique, our work pursues different goals. The primary goal of our work is to explore the possibility of use local information contained in a neighborhood of a pixel in images to improve performance of Gabor wavelet-based techniques. This information can be used to refine computation of Hamming Distances between images and make the combined algorithm more noise resistant compared to the traditional Gabor wavelet-based approaches.

\subsubsection{Ordinal Binary Coding and Combining Methodology}

Consider two iris templates $I$ and $I^{\prime}$ encoded to provide two binary iris codes $\mathbf{C}_{\text {Gabor }}^{I}$ and $\mathbf{C}_{G a b o r}^{I^{\prime}}$. To evaluate match or non-match using a traditional Gabor wavelet-based approach, the codes have to be initially aligned, and the total Hamming Distance (HD) normalized by the number of unoccluded pixels common to two iris codes is calculated. The alignment part as well as matching part can be easily refined. Since iris code contains information about both real and imaginary parts, for a certain pixel pair, for example, the pixel $p_{0}$ from templates $I$ and the pixel $p_{0}^{\prime}$ from templates $I^{\prime}$, HD can only take three possible values: 0 , 1 and 2 , since we have 2 bits to describe a value of each pixel. The two bits for pixel $p_{0}$ are $\Re_{p_{0}}$ and $\Im_{p_{0}}$. The two bits for pixel $p_{0}^{\prime}$ are $\Re_{p_{0}^{\prime}}$ and $\Im_{p_{0}^{\prime}}$. To provide an example we list all possible combinations and values that HD can take in Table 6.1. From here the normalized HD for each pixel pair can take only three values: $0,0.5$ and 1 . It can be interpreted as following:

0: Two pixels with their surroundings are similar.

0.5: The relation between two pixels is uncertain. 
1: Two pixels with their surroundings are different.

After analyzing this observation, we raised the following question. Would we benefit from refining the value of HD further? Our experimental analysis that we will present further has shown that using 3 bits or more by itself will not result in performance improvement. However, combination of refined HD and binary code that uses ordinal information contained in pixel values considerably improve the recognition performance of the Gabor wavelet-based approach.

Table 6.1: All possible Hamming distance outcomes for a pair of pixels $p_{0}$ and $p_{0}^{\prime}$ using log-Gabor binary encoding scheme

\begin{tabular}{|c|c|c|c|c|}
\hline HD & $\Re_{p_{0}}=0$ & $\Re_{p_{0}}=0$ & $\Re_{p_{0}}=1$ & $\Re_{p_{0}}=1$ \\
\cline { 1 - 5 } Normalized HD & $\Im_{p_{0}}=0$ & $\Im_{p_{0}}=1$ & $\Im_{p_{0}}=0$ & $\Im_{p_{0}}=1$ \\
\hline$\Re_{p_{0}^{\prime}}=0$ & 0 & 1 & 1 & 2 \\
$\Im_{p_{0}^{\prime}}=0$ & 0 & $\mathbf{0 . 5}$ & $\mathbf{0 . 5}$ & 1 \\
\hline$\Re_{p_{0}^{\prime}}=0$ & 1 & 0 & 2 & 1 \\
$\Im_{p_{0}^{\prime}}=1$ & $\mathbf{0 . 5}$ & 0 & 1 & $\mathbf{0 . 5}$ \\
\hline$\Re_{p_{0}^{\prime}}=1$ & 1 & 2 & 0 & 1 \\
$\Im_{p_{0}^{\prime}}=0$ & $\mathbf{0 . 5}$ & 1 & 0 & $\mathbf{0 . 5}$ \\
\hline$\Re_{p_{0}^{\prime}}=1$ & 2 & 1 & 1 & 0 \\
$\Im_{p_{0}^{\prime}}=1$ & 1 & $\mathbf{0 . 5}$ & $\mathbf{0 . 5}$ & 0 \\
\cline { 2 - 5 } & & & &
\end{tabular}

Given an image, consider an eight neighborhood of a pixel. The idea behind ordinal binary encoding is to make use of local information contained in neighboring pixel values. The total ordinal information can be expressed either using relative rank information or using pairwise ordinal relationship. We can represent relative relationship of pixels within 8-neighborhood using a $9 \times 9$ binary matrix. We provide an example in Fig. 6.1. An entry at $i$ th row and $j$ th column in the matrix will be set to one if the value of row head $p_{i-1}$ is larger than the column head $p_{j-1}$ and it will be set to zero if $p_{i-1}$ is equal to or smaller than the column head $p_{j-1}$. No values are usually assigned to the diagonal elements since the rank relationship for the same pixel is meaningless. Because of the symmetry of the matrix, the entire pixel relationship in 8-neighborhood is contained in the upper right (or lower left) triangle. Now, given a rank matrix, we can associate a 36-bit binary code generated using ordinal information with each pixel. 


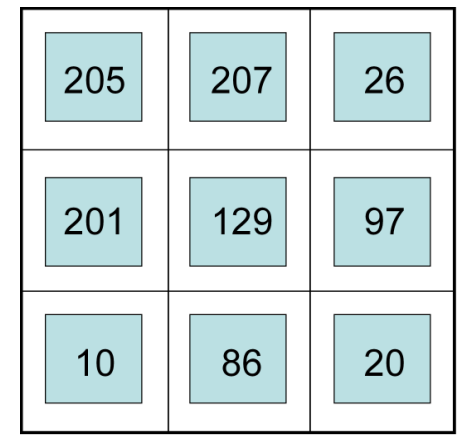

(a)

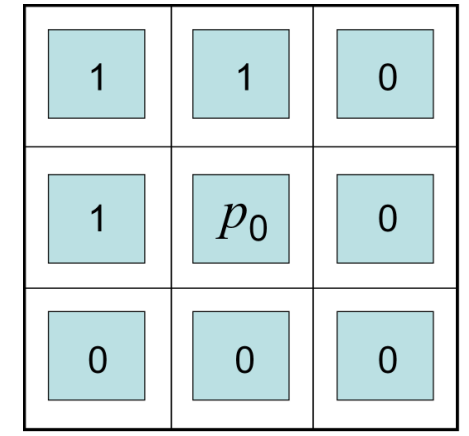

(b)

\begin{tabular}{|c|c|c|c|c|c|c|c|c|c|}
\hline$>$ & $\begin{array}{ll}P_{0} \\
(129) \\
\end{array}$ & $\begin{array}{l}P_{200} \\
2059 \\
\end{array}$ & $\begin{array}{l}P_{2} \\
(207)\end{array} \mid$ & $\begin{array}{l}P_{3} \\
(26) \\
\end{array}$ & $\begin{array}{l}P_{4} \\
\text { (2011) } \\
\end{array}$ & \begin{tabular}{|l|l|}
$P_{5}$ \\
$(97)$ \\
\end{tabular} & $\begin{array}{c}P_{6} \\
(10) \\
\end{array}$ & $\begin{array}{l}{ }_{(87} \\
886)\end{array}$ & $\begin{array}{l}P_{8} \\
(20) \\
\end{array}$ \\
\hline 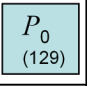 & & 0 & 0 & 1 & 0 & 1 & 1 & 1 & 1 \\
\hline \begin{tabular}{|l|}
$P_{1}$ \\
$2055)$ \\
\end{tabular} & 1 & & 0 & 1 & 1 & 1 & 1 & 1 & 1 \\
\hline$\left[\begin{array}{l}P_{2} \\
2027\end{array}\right]$ & 1 & 1 & & 1 & 1 & 1 & 1 & 1 & 1 \\
\hline \begin{tabular}{|l}
$P_{3}$ \\
(26) \\
\end{tabular} & 0 & 0 & 0 & & 0 & 0 & 1 & 0 & 1 \\
\hline \begin{tabular}{|l|}
$P_{4}$ \\
2011) \\
\end{tabular} & 1 & 0 & 0 & 1 & & 1 & 1 & 1 & 1 \\
\hline \begin{tabular}{|l}
$P_{5}$ \\
$(97)$ \\
\end{tabular} & 0 & 0 & 0 & 1 & 0 & & 1 & 1 & 1 \\
\hline \begin{tabular}{|l}
$\begin{array}{l}P_{6} \\
(10)\end{array}$ \\
\end{tabular} & 0 & 0 & 0 & 0 & 0 & 0 & & 0 & 0 \\
\hline \begin{tabular}{|l|}
$P_{7}$ \\
$(86)$ \\
\end{tabular} & 0 & 0 & 0 & 1 & 0 & 0 & 1 & & 1 \\
\hline $\begin{array}{l}P_{8} \\
200\end{array}$ & 0 & 0 & 0 & 0 & 0 & 0 & 1 & 0 & \\
\hline
\end{tabular}

(c)

Figure 6.1: LBP vs. LFOBC: (a) the intensity values of pixel $p_{0}$ and its neighbors, (b) LBP code: $\underline{110} \underline{10} \underline{000}$, (c) LFOBC code: $\underline{11010000} \underline{1000000} \underline{000000} \underline{11010} \underline{0000} \underline{000} \underline{11} \underline{0}$, or $\underline{00101111} 0111111 \underline{111111} 00101 \underline{1111} 111 \underline{00} \underline{1}$. 
The described above encoding procedure is called local full ordinal binary code (LFOBC) in this work. It is similar to local binary pattern (LBP) when parameters are selected to be $\mathrm{P}=8, \mathrm{R}=1.0$. Here $\mathrm{P}$ is the number of neighbors and $\mathrm{R}$ is the radius of the circle where the neighbors are sampled. If the value of the center pixel is subtracted from the values of the neighbors, the local binary pattern can be calculated by considering only the signs of the differences $[105,106]$. The comparison of LBP and LFOBC methods is presented in Fig. 6.2. From this specific example, it is clear that LBP code is presented by the first column of the LFOBC code if the lower left triangle is considered, or the inverse (1 to 0 and 0 to 1 ) of the first row of the LFOBC code if the upper right triangle is selected.

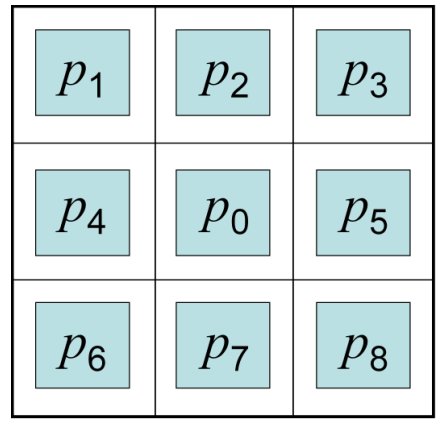

(a)

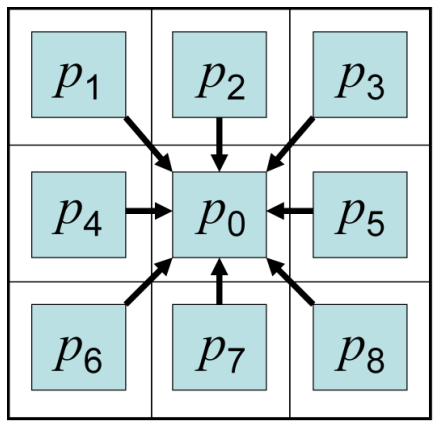

(b)

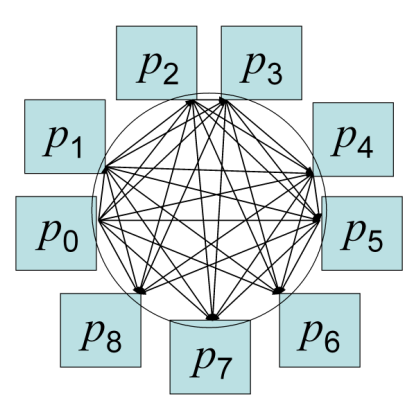

(c)

Figure 6.2: LBP vs. LFOBC: (a) the pixel $p_{0}$ and its neighbors, (b) the calculation of LBP, (c) the calculation of LFOBC.

As we mentioned earlier, LFOBC may be easily combined with Gabor wavelet-based encoding. We do this when we compute the normalized HD for each pixel pair after performing the alignment of two iris codes. While we keep unchanged zeros and ones that normalized HD per pixel can take, when HD takes value 0.5 (see Table 6.1 for illustration), we replace it with a refined value of normalized HD. It provides us with a possibility to make a soft decision. The normalized HD of LBP codes for a pair of pixels, $\mathbf{C}_{L B P}^{p_{0}}, \mathbf{C}_{L B P}^{p_{0}^{\prime}}$, is calculated as

$$
\mathbf{D}_{L B P}=\frac{\operatorname{XOR}\left(\mathbf{C}_{L B P}^{p_{0}}, \mathbf{C}_{L B P}^{p_{0}^{\prime}}\right)}{8},
$$

where XOR is the binary exclusive OR operation. The normalized HD of LFOBC codes for a pair of pixels, $\mathbf{C}_{L F O B C}^{p_{0}}, \mathbf{C}_{L F O B C}^{p_{0}^{\prime}}$, is calculated as

$$
\mathbf{D}_{L F O B C}=\frac{\operatorname{XOR}\left(\mathbf{C}_{L F O B C}^{p_{0}}, \mathbf{C}_{L F O B C}^{p_{0}^{\prime}}\right)}{36} .
$$


Table 6.2 provides details on calculation of the normalized HD.

Table 6.2: The new normalized Hamming distance calculation scheme for a pair of pixels $p_{0}$ and $p_{0}^{\prime}$ seamlessly integrate the ordinal binary codes into the log-Gabor binary code

\begin{tabular}{|c|c|c|c|c|}
\hline $\begin{array}{c}\text { Normalized } \\
\text { HD }\end{array}$ & $\begin{array}{l}\Re_{p_{0}}=0 \\
\Im_{p_{0}}=0\end{array}$ & $\begin{array}{l}\Re_{p_{0}}=0 \\
\Im_{p_{0}}=1\end{array}$ & $\begin{array}{l}\Re_{p_{0}}=1 \\
\Im_{p_{0}}=0\end{array}$ & $\begin{array}{l}\Re_{p_{0}}=1 \\
\Im_{p_{0}}=1\end{array}$ \\
\hline $\begin{array}{l}\Re_{p_{0}^{\prime}}=0 \\
\Im_{p_{0}^{\prime}}=0\end{array}$ & 0 & $\begin{array}{l}\mathrm{D}_{L F O B C} \\
\text { or } \mathrm{D}_{L B P}\end{array}$ & $\begin{array}{l}\mathrm{D}_{L F O B C} \\
\text { or } \mathbf{D}_{L B P}\end{array}$ & 1 \\
\hline $\begin{array}{l}\Re_{p_{0}^{\prime}}=0 \\
\Im_{p_{0}^{\prime}}=1\end{array}$ & $\begin{array}{l}\mathbf{D}_{L F O B C} \\
\text { or } \mathrm{D}_{L B P}\end{array}$ & 0 & 1 & $\begin{array}{l}\mathrm{D}_{L F O B C} \\
\text { or } \mathrm{D}_{L B P}\end{array}$ \\
\hline $\begin{array}{l}\Re_{p_{0}^{\prime}}=1 \\
\Im_{p_{0}^{\prime}}=0\end{array}$ & $\begin{array}{l}\mathrm{D}_{L F O B C} \\
\text { or } \mathrm{D}_{L B P}\end{array}$ & 1 & 0 & $\begin{array}{l}\mathrm{D}_{L F O B C} \\
\text { or } \mathrm{D}_{L B P}\end{array}$ \\
\hline $\begin{array}{l}\Re_{p_{0}^{\prime}}=1 \\
\Im_{p_{0}^{\prime}}=1\end{array}$ & 1 & $\begin{array}{l}\mathrm{D}_{L F O B C} \\
\text { or } \mathrm{D}_{L B P}\end{array}$ & $\begin{array}{l}\mathbf{D}_{L F O B C} \\
\text { or } \mathbf{D}_{L B P}\end{array}$ & 0 \\
\hline
\end{tabular}

Since both a Gabor wavelet-based binary code and a local ordinal binary informationbased code relies on local information in iris images, the estimated occlusion mask can be easily modified for use in the combined encoding scheme.

In this work, we rely on Libor Masek's implementation [41,45] of the log-Garbor filter based iris algorithm. We use it as a basis for our developments, since there is no publicly available version of Daugman's algorithm.

To conclude on the performance of the proposed methods, we carry a number of experiments.

\subsubsection{Simulated Camera Effects}

To evaluate the resistance of the proposed combined scheme to nonidealities in images, we synthetically degrade images by adding simulated effects. The details on simulation of camera and environmental effects that can be observed in iris images can be found in $[58,70]$.

Three effects including noise, out-of-focus blur and motion blur are individually simulated. We apply these effects to three types of patterns: random patterns, natural textures and unwrapped iris templates. For consistency we normalize all images and templates to be of size $[20 \times 240]$. The performance of different methods was evaluated using the "decidabil- 
ity" metric $d^{\prime}[8]$ given by

$$
d^{\prime}=\frac{\left|\mu_{1}-\mu_{2}\right|}{\sqrt{\frac{\sigma_{1}^{2}+\sigma_{2}^{2}}{2}}},
$$

where $\mu_{1}$ and $\mu_{2}$ are the mean of genuine and imposter HD distances, and $\sigma_{1}$ and $\sigma_{2}$ are the variances of genuine and imposter HD distances. We generated 100 random noisy patterns using uniform distributed intensity values from 0 to 255 with independent intensity values from pixel to pixel. We selected 100 textures from Brodatz dataset of natural textures [43]. For each selected image, the top left corner of the size $[20 \times 240]$ is selected. We also selected 100 unwrapped iris templates obtained by processing images from CASIA database [76]. Examples of the images and templates are provided in Fig. 6.3.

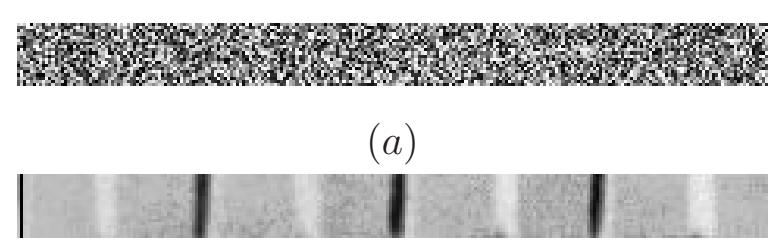

(b)

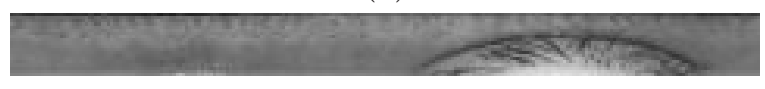

(c)

Figure 6.3: Testing templates: (a) random pattern, (b) natural image, (c) iris template.

\section{Noise}

First, we evaluate the robustness of the proposed schemes to additive noise. We simulate random noise that can be observed in images by adding a realization of a uniformly or Gaussian distributed random variable at five distinct levels parameterized by five distinct values of standard deviation (from 14.7 to 73.6 taken with the step 14.7). The intensities in generated noisy images were truncated to lie in the range $[0,255]$. The results of the experiments are summarized in Tables 6.3, 6.4 and 6.5. We can see that local ordinal encoding methods are robust to the noise for local patterns with high variance, such as random patterns, but relatively weak for smooth patterns, such as natural textures. LogGabor-based methods perform well on natural patterns but, as observed, are not robust in the presence of high level noise. It can be also observed that the proposed combined scheme 
including log-Gabor-based technique and the local ordinal code performs relatively well in the presence of noise.

Table 6.3: Recognition capabilities of five encoding techniques in the presence of random noise (case of uniform distributed noise and random textures

\begin{tabular}{|c|c|c|c|c|c|}
\hline \multirow{2}{*}{$d^{\prime}$} & \multicolumn{5}{|c|}{ Standard Deviation of the Noise } \\
\cline { 2 - 6 } & 14.7 & 29.5 & 44.2 & 58.9 & 73.6 \\
\hline \multicolumn{5}{|c|}{ Uniform } \\
\hline log-Gabor only & 50.4 & 33.5 & 28.0 & 17.0 & 12.6 \\
\hline LBP only & 94.2 & 49.4 & 53.5 & 23.7 & 17.8 \\
\hline LFOBC only & 105.8 & 52.2 & 60.6 & 24.5 & 18.4 \\
\hline log-Gabor+LBP & 55.7 & 38.3 & 37.5 & 20.4 & 15.3 \\
\hline log-Gabor+LFOBC & 54.9 & 38.0 & 37.3 & 20.4 & 15.3 \\
\hline \multicolumn{5}{|c|}{ Gaussian } \\
\hline log-Gabor only & 50.2 & 37.7 & 28.4 & 21.5 & 16.4 \\
\hline LBP only & 94.5 & 71.3 & 54.7 & 41.3 & 32.4 \\
\hline LFOBC only & 105.7 & 80.5 & 61.2 & 45.9 & 35.7 \\
\hline log-Gabor+LBP & 55.6 & 47.3 & 37.7 & 28.9 & 22.3 \\
\hline log-Gabor+LFOBC & 54.9 & 46.9 & 37.5 & 29.0 & 22.3 \\
\hline
\end{tabular}

\section{Out-of-focus Blur}

In this work, the out-of-focus blur is simulated using circular averaging filters [107]. We involve five defocus levels, from 1 to 5 , corresponding to the radius, $r$, measured in pixels of the averaging filter. Including the original image, each class has total 6 blurred copies. The results of performance evaluation for this case are presented in Table 6.6. It is easy to see that out-of-focus blur reduces the contrast of the image. This effect substantially degrades the performance of individual ordinal information-based methods as the level of blur increases. Natural images are less sensitive to out-of-focus blur compared to randomly generated images. The combined log-Gabor and LFOBC encoding do not result in substantial improvement because of loss of the local ordinal information.

\section{Motion Blur}

In this work, we consider only linear motion blur. This effect is simulated by modeling two parameters: direction of smear, denote it by $\theta$, and pixel-smear length amount while 
Table 6.4: Recognition capabilities of five encoding techniques in the presence of random noise (case of uniform distributed noise and Brodatz textures)

\begin{tabular}{|c|c|c|c|c|c|}
\hline \multirow{2}{*}{$d^{\prime}$} & \multicolumn{5}{|c|}{ Standard Deviation of the Noise } \\
\cline { 2 - 6 } & 14.7 & 29.5 & 44.2 & 58.9 & 73.6 \\
\hline \multicolumn{5}{|c|}{ Uniform } \\
\hline log-Gabor only & 4.92 & 3.64 & 3.04 & 2.67 & 2.40 \\
\hline LBP only & 2.84 & 2.31 & 2.10 & 1.98 & 1.88 \\
\hline LFOBC only & 2.99 & 2.45 & 2.25 & 2.15 & 2.09 \\
\hline log-Gabor+LBP & 5.11 & 3.75 & 3.15 & 2.78 & 2.50 \\
\hline log-Gabor+LFOBC & 5.17 & 3.78 & 3.17 & 2.80 & 2.52 \\
\hline \multicolumn{6}{|c|}{ Gaussian } \\
\hline log-Gabor only & 4.94 & 3.69 & 3.09 & 2.75 & 2.52 \\
\hline LBP only & 2.90 & 2.35 & 2.11 & 1.95 & 1.87 \\
\hline LFOBC only & 3.06 & 2.48 & 2.26 & 2.12 & 2.07 \\
\hline log-Gabor+LBP & 5.14 & 3.80 & 3.19 & 2.84 & 2.63 \\
\hline log-Gabor+LFOBC & 5.20 & 3.84 & 3.21 & 2.86 & 2.64 \\
\hline
\end{tabular}

the width of the Point Spread Function (PSF) is kept constant, 1 pixel wide [107]. In our experiments, we fix the direction parameter $\theta$ at 45 degrees. Motion strength, from 1 to 5, corresponds to the length of the blur in pixels. For each class we generated 5 blurred copied of undistorted image. The dependence of the d-prime $\left(d^{\prime}\right)$ measure on the increased level of motion blur for five distinct encoding techniques is summarized in Table 6.7. The effect of motion blur on performance is not as pronounced as the effect of out-of focus blur.

\subsubsection{Recognition Performance}

To evaluate recognition capabilities of different encoding schemes we involve a subset of CASIA-3 dataset [76]. A subset of 750 images from 75 classes (10 images per class) were selected from "CASIA-IrisV3-Interval" subdirectory. This subdirectory contains images in JPEG format. We used a broad range angles from +15 degree to -15 degree to compensate for the head tilt. All iris templates are normalized to be of size $[20 \times 240]$.

The ROC curves for different encoding schemes are shown in Fig. 6.4. To further compare the performance of the encoding schemes we summarize the values of $d^{\prime}$ and EER in Table 6.8. The results indicate that the performance can be considerably improved by introducing additional bits for ambiguous pixel pairs by using local ordinal relationship information. For 
Table 6.5: Recognition capabilities of five encoding techniques in the presence of random noise (case of uniform distributed noise and iris textures)

\begin{tabular}{|c|c|c|c|c|c|}
\hline \multirow{2}{*}{$d^{\prime}$} & \multicolumn{5}{|c|}{ Standard Deviation of the Noise } \\
\cline { 2 - 6 } & 14.7 & 29.5 & 44.2 & 58.9 & 73.6 \\
\hline \multicolumn{5}{|c|}{ Uniform } \\
\hline log-Gabor only & 3.85 & 1.77 & 0.82 & 0.33 & 0.05 \\
\hline LBP only & 2.52 & 1.15 & 0.67 & 0.39 & 0.29 \\
\hline LFOBC only & 2.59 & 1.31 & 0.81 & 0.52 & 0.41 \\
\hline log-Gabor+LBP & 4.17 & 2.58 & 1.71 & 1.14 & 0.79 \\
\hline log-Gabor+LFOBC & 4.17 & 2.59 & 1.73 & 1.16 & 0.82 \\
\hline \multicolumn{6}{|c|}{ Gaussian } \\
\hline log-Gabor only & 3.87 & 1.86 & 0.89 & 0.40 & 0.12 \\
\hline LBP only & 2.54 & 1.24 & 0.67 & 0.40 & 0.29 \\
\hline LFOBC only & 2.61 & 1.38 & 0.82 & 0.54 & 0.41 \\
\hline log-Gabor+LBP & 4.15 & 2.67 & 1.77 & 1.20 & 0.87 \\
\hline log-Gabor+LFOBC & 4.16 & 2.68 & 1.79 & 1.23 & 0.90 \\
\hline
\end{tabular}

Table 6.6: Performance comparison for out-of-focus blur

\begin{tabular}{|c|c|c|c|}
\hline$d^{\prime}$ & 1 & 3 & 5 \\
\hline log-Gabor only & 3.5457 & 6.4886 & 5.1957 \\
\hline LBP only & 1.2150 & 2.7057 & 1.6291 \\
\hline LFOBC only & 1.3735 & 2.8741 & 1.7449 \\
\hline log-Gabor+LBP & 3.2299 & 6.3206 & 4.1057 \\
\hline log-Gabor+LFOBC & 3.2258 & 6.3881 & 4.1549 \\
\hline
\end{tabular}

stand alone scenario as well as in combination with the log-Gabor code, LFOBC slightly outperforms LBP according to $d^{\prime}$. However, as shown in the rows 4 and 5 in Table 6.8, LFOBC has a larger $d^{\prime}$ but also a larger value of EER compared to the LBP when they were combined with the log-Gabor code. The complexity, both for the purpose of storage and for the purpose of computational efficiency, 36 bits vs. 8 bits, may be a point of trade-off of performance vs. complexity.

\subsection{Median Filter Based Iris Encoding Technique}

Iris-based human recognition is very attractive because of the high accuracy achievable. However, existing encoding methods are unable to handle iris images acquired when the 
Table 6.7: Performance comparison for motion blur

\begin{tabular}{|c|c|c|c|}
\hline$d^{\prime}$ & 1 & 3 & 5 \\
\hline log-Gabor only & 5.1394 & 12.2236 & 9.5115 \\
\hline LBP only & 3.1893 & 5.2554 & 3.5827 \\
\hline LFOBC only & 3.7063 & 6.0333 & 3.8453 \\
\hline log-Gabor+LBP & 6.1813 & 15.2305 & 6.7801 \\
\hline log-Gabor+LFOBC & 6.5999 & 15.6932 & 6.7893 \\
\hline
\end{tabular}

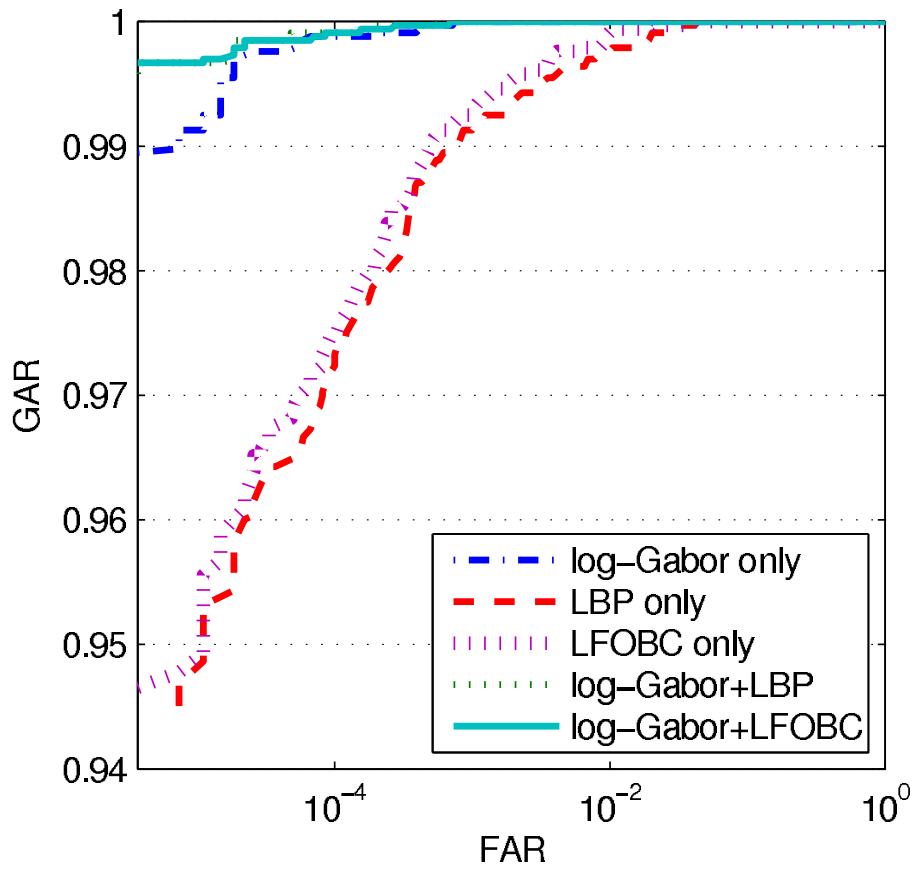

Figure 6.4: Performance comparison of different coding schemes for CASIA-3 dataset.

ambient lighting is non-uniform. In this section we propose a novel encoding technique which can handle images acquired under such conditions. The method is based on 2D median filters, which are commonly used as denoising tools in image processing domain, and uses their nonlinear characteristics to generate binary codes from gray scale iris images.

\subsubsection{Introduction}

Both the 2D Gabor filter and the 1D log-Gabor filter are directional. For the 2D Gabor filter the sinusoidal part determines the dominant direction. Different choices of the direction will result in different recognition performance, although banks of different filters can solve 
Table 6.8: Performance comparison of five encoding schemes using d-prime $\left(d^{\prime}\right)$ and EER

\begin{tabular}{|c|c|c|}
\hline & $d^{\prime}$ & EER \\
\hline log-Gabor only & 7.7129 & $5.4 \times 10^{-4}$ \\
\hline LBP only & 5.2041 & 0.0042 \\
\hline LFOBC only & 5.3768 & 0.0037 \\
\hline log-Gabor+LBP & 8.5204 & $3.0 \times 10^{-4}$ \\
\hline log-Gabor+LFOBC & 8.6560 & $3.1 \times 10^{-4}$ \\
\hline
\end{tabular}

this problem to some degree. Generally the filters are applied in a horizontal direction in the unwrapped image (i.e. circumferentially in the original image).

Based on our observation Gabor filtering techniques are not very robust to uneven illumination since illumination change is treated largely as signal rather than noise. Ma et al. [13] try to deal with non-uniform brightness by subtracting the local mean. But low illumination will also cause low contrast in addition to low intensity values. Although they employ a subsequent histogram equalization based image enhancement, this may amplify the noise at the same time as it increases contrast. This is one of the main reasons why we propose a median filter based encoding technique: the image enhancement and image encoding can be performed at the same time.

There are a variety of other techniques which have been used to generate binary iris codes. These include modified log-Gabor filters [9], non-local comparisons [10], dyadic wavelet transforms [13], derivative-of-Gaussians [12], Laplacian-of-Gaussian filters [12], [11], discrete cosine transforms [14], wavelet packets [15], [16], [17], and quotient thresholding techniques [18]. Our method falls into the local ordinal measures category [10] and is similar to the thresholding approach of [18]. This method used local histogram equalization to compensate non-uniform illumination, and adaptive quotient thresholding to keep the ratio between the foreground and background constant. Based on experiments, they found that the best decision ratio was 0.33 . Yet we believe local histogram equalization is not necessary if an adaptive threshold can be determined for each pixel. Thus in our approach a large window median filter is used to compensate for non-uniform illumination instead, as will be described below. 


\subsubsection{Proposed Algorithm}

The goal is to encode the unwrapped iris image so that the resulting binary pattern is unique for each individual's iris. Furthermore, because Hamming distance is used to measure similarity, patterns with approximately half 0's and half 1's are preferred. This prompted us to examine median filters since, statistically, the median is designed to separate the data exactly in half.

\section{D Median Filters}

2D median filtering is a nonlinear operation. Each pixel value in an image is replaced by the median value computed in a local neighborhood around the pixel. In image processing this is more effective than convolution in simultaneously reducing noise while preserving edges [99]. For unwrapped iris images, the neighborhood should wrap around at the boundaries (i.e. the right side is connected to the left side in a ring).

The block diagram of median filtering based encoding can be seen in Fig. 6.5. The original image is shown at the top of the plot and results with different window sizes are shown below. The size of the local neighborhood is identified in the lower right corner of each image. It is clear that median filters with a larger window sizes capture the gross illumination variations better than filters with a smaller window size.

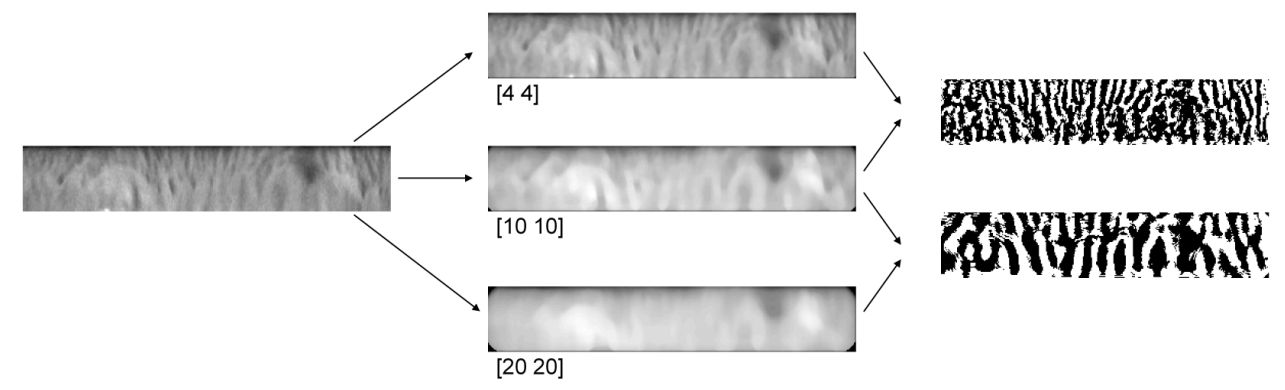

(a)

(b)

(c)

Figure 6.5: The block diagram of median filtering based encoding: (a) the original iris pattern; (b) 2D median filter results; and (c) the binary code generated from the filtered results.

The encoding method introduced here is fairy intuitive and simple. First a median filtered result with a small window size $m \times m$ is generated to capture small intensity variations. Next 
another one using bigger window size, $n \times n$ where $n>m$, is generated to compensate for brightness differences. The difference between is a less noisy and highly distinctive feature. Thus the comparison result between the two at each pixel can be used as binary code for this image.

\section{Scale Selection}

For optimal performance we have selected three particular window sizes as mentioned in Fig. 6.5. This choice comes mainly from visual inspection and experimentation. A variety of settings may give similar results. For small window sizes (total pixel count less than 20), even dimensions are recommended since otherwise a clearly unequal distribution of 0 s and 1s may appear. When the resolution of unwrapped images is $64 \times 360$ we found that the combination of [4 4$]$ and $\left[\begin{array}{ll}10 & 10\end{array}\right]$ captured the smaller texture patterns (e.g. fibers), while the combination of [10 10] and [20 20] captured the larger texture patterns (e.g. bundles, crypts).

In many cases a smoothing procedure applied to the original image before encoding can enhance the recognition performance by reducing the effects of noise. The unwrapped image may optionally be smoothed using an averaging filter over a square block of $9 \times 9$ pixels (applied with wraparound). Floating point rather than integer intensity values are preferred for the smoothed image.

\subsection{Cancelable Iris Biometric}

A person only has two irises. If his pattern is stolen he quickly runs out of alternatives. Thus methods that protect the true iris pattern need to be adopted in practical biometric applications. In particular, it is desirable to have a system that can generate a new unique pattern if the one being used is lost, or generate different unique patterns for different applications to prevent cross-matching. For backwards compatibility, these patterns should look like plausible irises so they can be handled with the same processing tools. However, they should also non-invertibly hide the true biometric so it is never exposed, or even stored. In this section four such cancelable biometric methods are proposed that work with conventional 
iris recognition systems, either at the unwrapped image level or at the binary iris code level.

\subsubsection{Introduction}

Iris is a strong biometric in term of recognition performance, both theoretically and empirically. However, traditional iris biometric schemes are weak with respect to privacy and certain aspects of security. Although the digital encoding of an iris pattern can be encrypted, if the encryption is ever broken the true biometric is "lost" forever. Or, through the use of a trojan horse type attack, the true biometric can be harvested at the matching stage when the system has already decrypted it. If the true iris is disclosed, it can conceivably used in a replay attack to break into the system. Even worse, the same true iris could possibly be used to break into other, unrelated systems that were also keyed to the person's iris. This is a security problem, for sure, and may also be a liability problem in case of consequential damages. In as much as the same iris can be used to illicitly gain access to multiple databases, such database crossmatching may also be performed for gathering business intelligence or by court order. The individual would have far more privacy if each of the databases was keyed to incompatible "fake" irises derived from the true iris of the user.

In our new "cancelable" iris scheme, the user's true pattern never has to leave the client computer. After the necessary biometrics preprocessing, such as segmentation and encoding, the unwrapped iris pattern image or iris code is intentionally distorted using a non-invertible transform. These new versions of the iris are secure because the original iris pattern can not be recovered even from the stored (or transmitted) representation. They are also cancelable because another totally different pattern or code can be generated by the transform procedure by simply supplying a different set of distortion parameters. In this way one or two irises can be multiplied into thousands of different virtual irises.

Cancelable biometrics has been proposed by Ratha et al. [108] and has proved very successful in fingerprint biometric [109]. There are other related bodies of work that try to provide similar benefits for irises. Davida et al. used hash functions to protect sensitive user information [110]. Juels et al. introduced a fuzzy commitment scheme that can be applied to iris codes [111]. Chong et al. proposed two iris verification schemes which take cancelable 
biometrics into account [112] [113]. However in their work they emphasize the performance improvement that can be achieved rather than the cancelability of the iris biometric. Hao et al. tried to combine standard cryptographic techniques with iris codes to enhance the total security level of the system [114]. But the actual iris biometric is still vulnerable if the

decoding is done at the server. If, instead, only the reproduced key $\hat{K}$ will be sent to the server, then the security is at a similar level to just the password key. If both the key and the iris biometric are used, the false reject rate will increase since both the recovered key and Hamming match distance are subject to noise from the iris pattern.

\subsubsection{Methodology}

New cancelable iris methods operate on the unwrapped image or the resulting binary feature vector. The first two methods use only the information from the biometric itself, while the second two methods use additional external random information for distortion. In each case the transforms used are non-invertible and revocable. The first criterion is essential to security, while the second guards against loss of identity and there can be more than one biometrics application.

\section{Registration-free Transforms}

The first method, called GRAY-COMBO, involves shifting and combining rows in the unwrapped iris image. This illustrated in Fig. 6.6. Note that this is just a sketch of the idea; in real system more rows and columns would be used. First we shift rows circularly in the horizontal direction using random offsets (part of the transform key). Then two randomly selected rows (another part of the key) are combined together using an operator like addition or multiplication. A single row can conceivably be used in more than one combination, although in the example here (and in the later discussion of the performance) each row only is used only once. A similar transform can alternatively be applied to the binary iris code. This method, referred to as BIN-COMBO, is illustrated in Fig. 6.7. The row shifting is similar, but the combination operation needs to be changed to XOR or XNOR. Both these method are non-invertible: all original pixel intensities or bit strings are damaged. So the 
original rows cannot be recovered. Note that, like the addition or multiplication used in GRAY-COMBO, XOR is also not invertible if one knows only the result (e.g. 0 can come from 1 XOR 1 or from 0 XOR 0 ). Also note that neither of these methods requires the iris to be put into some known canonical orientation as the row shifts will be the same no matter how the captured iris image is rotated. This is why we call these transforms registration-free. Furthermore these methods have the added advantage that the same back-end processing and matching tools can continue to be used. The one problem with these two methods is that the amount of valid iris area generally decreases. In an iris image, often a substantial portion of the pattern is occluded by eyelids or eyelashes. When shifting the rows for these two transforms the validity mask must also be shifted. The masks from two rows are then combined so that the portion of the transformed image that is valid will be smaller than the original. The invalid area can be doubled, or even worse, if a highly occluded row is used several times. Smaller shift values will generally invalidate less of the image than larger shifts, but this sacrifices some key strength. So these methods are most useful when good quality (low occlusion) images are available.

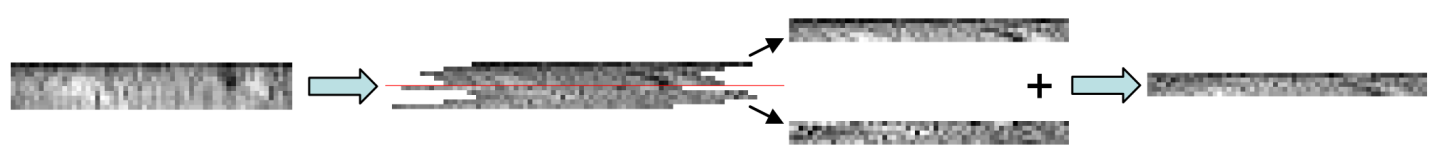

(a)

(b)

(c)

(d)

Figure 6.6: The block diagram of GRAY-COMBO: (a) the original iris pattern; (b) random shifting; (c) random split (in this case, just split to the top half and the bottom half); and (d) combine together (using plus here).

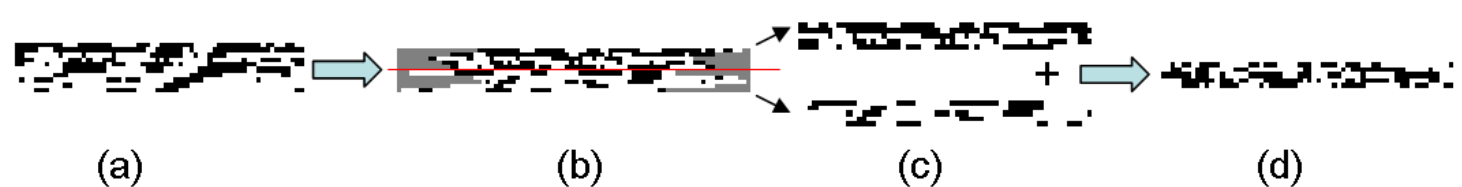

Figure 6.7: The block diagram of BIN-COMBO: (a) the original iris pattern; (b) random shifting; (c) random split (in this case, just split to the top half and the bottom half); and (d) combine together (using XOR here). 


\section{Salting Approach}

Another way of generating a cancelable iris is by mixing in a totally artificial pattern. This can be pure random noise, a randompattern with iris-like texture, or a synthetic iris pattern [115]. For gray-scale unwrapped irises typically the two images are combined pixelwise using either addition or multiplication. This method, GRAY-SALT, is illustrated in Fig. 6.8. A similar technique can also be applied to the final binary iris code. This BIN-SALT method has the advantage that, for legacy systems, the iris code is often more accessible than the unwrapped image is. Typically it combines the original iris code with the key pattern using XOR (again, non-invertible) as shown in Fig. 6.9. Unlike the COMBO methods, there is no shrinking of the valid iris area since the key pattern that is being mixed in is valid everywhere. However these methods suffer from an alignment problem. If the fixed pattern is applied at the wrong rotation it will not generate a signature similar to what as was enrolled (with a different orientation). Thus the matcher needs to try all plausible rotations of the original iris before applying this transform. Note that, since the position of the key is fixed, it is only the query image or code that needs to be aligned in order to find the best match.

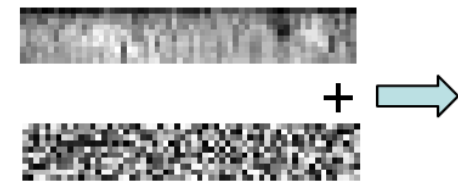

(a)

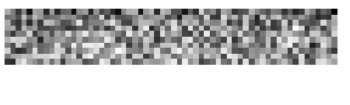

(b)

Figure 6.8: The block diagram of GRAY-SALT: the original iris pattern and the random noise pattern are combined together to generate the new cancelable iris pattern.

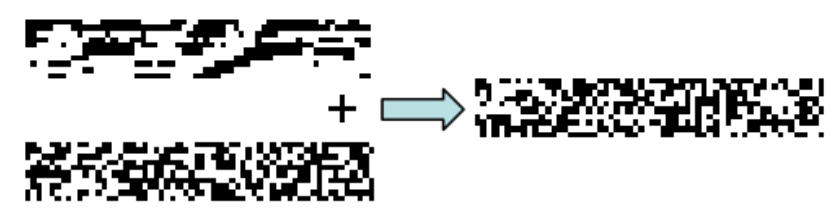

(a)

(b)

Figure 6.9: The block diagram of BIN-SALT: the original iris code and the random binary noise pattern are combined together to generate the new cancelable iris code.

Although only a non-invertibly transformed version of the iris needs to be stored, the 
alignment condition means that the true iris (and the key) need to be submitted to the matcher. This opens the possibility that one or both might be stolen over the network or, assuming encrypted transmission, after decrypting at the server. Thus extra secure effort needs to be taken.

\subsection{Conclusions}

Three different approaches to improve the performance of the iris biometric system from the aspect of encoding were introduced in this chapter. They are a local ordinal binary extension, median filter based binary iris code and "cancelable iris biometric" which can protect the private iris information. 


\section{Chapter 7}

\section{Adaptive Biometric Authentication}

By combining quality measures (discussed in Ch. 4) and an encoding algorithm, we can arrive to a number of methods to improve the performance of an iris biometric system. In this chapter, three methods to improve the performance of biometric matchers based on vectors of quality measures associated with biometric samples are described. The first two methods select samples and matching scores based on predicted values of Quality of Sample (QS) index (defined here as d-prime $d^{\prime}$ ) and Confidence in matching Scores (CS), respectively. The third method treats quality measures as weak but useful features for discrimination between genuine and imposter matching scores. The unifying theme for the three methods consists of a nonlinear mapping between quality measures and the predicted values of QS, CS, and combined quality measures and matching scores, respectively. The proposed methodology is generic and is suitable for any biometric modality. The experimental results reported show significant performance improvements for all the three methods when applied to iris biometrics.

\subsection{Introduction}

The most common definition of biometric sample quality is at the image or signal level. A quality checking block is introduced into every biometric system to ensure that enrolled image/signals have sufficient quality to be further processed.

Many recent biometric systems extract a vector of quality measures. The components of 
a vector of quality measures, however, rarely carry equal weight in terms of their relationship to the performance of the matcher. In practical applications (such as US Visit program), it is required to keep a single biometric quality measure in order to decide if biometric samples are suitable for further processing and matching. Research questions should thus be concerned with (i) what quality measures to use; (ii) how to combine multiple quality measures into a single quality index without losing the information that the vector of quality measures contains; and (iii) how to use this vector to improve performance of biometric systems? This chapter addresses all those questions.

Most of the quality based matchers described in the literature involve biometric sample quality at the matching stage by concatenating matching scores due to the original matcher and quality measures. These matchers are known as Q-stack classifiers [116, 117]. In spite of the fundamental theory presented in these works in support of Q-stack classifiers, the improvement of performance is marginal, if at all (see [118], [116]). More noticeable improvements are reported for Q-stack classifiers operating on multiple algorithms or multiple matchers $[117,118]$.

This chapter suggests several methods on the use of biometric sample quality to improve the performance of a single matcher. It targets two main applications for quality measures: 1) to improve performance of a matcher by predicting its QS index or CS score and using them to decide if the underlying biometric sample should be retained or discarded 2) to design a nonlinear matcher that treats a vector of quality measures as a set of weak features.

The remainder of the chapter is organized as follows. Sec. 7.2 describes the three proposed methods. Sec. 7.3 describes the data sets used and presents experimental results. Sec. 7.4 summarizes the contributions.

\subsection{Methodology}

This chapter advocates the use of predictive tools for the design of quality enhanced matchers. The tools proposed predict a set of quality measures and scores. In each of the three methods described below, the functional relationship between vectors of quality measures and the predicted (estimated) measures is not known and has to be modeled. The 
modeling problem is stated as a multivariate regression problem:

$$
Y=f\left(X_{1}, \ldots, X_{K}\right),
$$

where $f$ is a multivariate adaptive mapping, variable $Y$ is the estimated output variable, i.e. that characterizes the overall quality, confidence in matching score, or quality enhanced decision, and $X_{1}, \ldots, X_{K}$ is a vector of $K$ input (predictive) variables, such as a vector of quality measures for a biometric sample or a concatenated vector of quality measures and matching scores. Since the true relationship between the input and output variables is not known, it is estimated using a set of labeled training data. The multivariate adaptive mapping $f(\cdot)$ can be implemented using a variety of multivariate functions and systems. The results reported are obtained using a feed forward neural network (FFNN) (see Sec. 3).

\subsubsection{Quality of Sample (QS)}

Here we suggests a single quality index characterizing the overall quality of a biometric sample. Consider a set of biometric samples. Associated with these data are vectors of quality measures. For example, for iris biometrics a matcher may be designed to implement Hamming Distance (HD), while the quality vector may be composed of ten quality measures: 1) iris segmentation score, 2) pupil segmentation score, 3) blur, 4) illumination, 5) dilation, 6) interlacing, 7) lighting, 8) occlusion, 9) off-angle and 10) pixel count (see [119] for details). These data can be used to design a single quality index by fusing the entries of the vector. The main design requirement is that the values of the single quality index must be related to the performance of the original matcher.

We propose to use d-prime $\left(d^{\prime}\right)$ index as the combined quality index. This index is related to the relative entropy, which is asymptotically related to the performance of the matcher. Since the true distributions, means and variances of matching scores are not known, they are estimated using available labeled data. All labeled data are subdivided into two nonoverlapping sets: training set and testing set. $Q S_{A}$, the overall quality index of sample $A$, is

$$
Q S_{A}=\frac{\mid m(\text { Imp. Scores })_{A}-m(\text { Gen. Scores })_{A} \mid}{\sqrt{\operatorname{var}(\text { Imp. Scores })_{A}+\operatorname{var}(\text { Gen. Scores })_{A}}},
$$


where $m(\cdot)_{A}$ and $\operatorname{var}(\cdot)_{A}$ are the sample mean and sample variance of genuine and imposter scores formed by involving the sample $A$.

Using the labeled training set $Q S$ is estimated for every biometric sample. This requires that a set of genuine matching scores and a set of imposter matching scores involving the same biometric sample be formed. For unlabeled biometric samples this task becomes almost impossible. However, having quality vectors associated with each biometric sample makes it possible to predict the $Q S$ of unlabeled data. The $Q S$ can be obtained as the output parameter of a nonlinear multivariate adaptive mapping applied to a vector of quality measures (input parameters). Let $\mathbf{Q}_{A}=\left[Q_{A, 1}, \ldots, Q_{A, K}\right]^{T}$ be a vector of $K$ quality measures characterizing a biometric sample $A$. The superscript $T$ indicates the transpose operation. Let $f_{Q S}(\cdot)$ be a nonlinear multivariate adaptive mapping that maps a vector of quality measure $\mathbf{Q}_{A}$ into the quality index $Q S_{A}$. Let $\hat{f}_{Q S}(\cdot)$ be its estimated version. Then $Q S_{A}$ is

predicted as $Q S_{A}=\hat{f}_{Q S}\left(\mathbf{Q}_{A}\right)$. The predicted value of $Q S$ can be then used to decide if the underlying biometric sample should be retained or discarded to improve the performance of the original matcher.

\subsubsection{Confidence in Scores (CS)}

The second method evaluates the confidence level assigned to matching scores associated with a pair of biometric samples. The confidence in genuine and imposter scores (CS) is defined for an iris recognition system:

$$
\begin{gathered}
C S_{G}= \begin{cases}0, & H D_{G}<Q\left(H D_{G}\right)_{x}, \\
-\frac{H D_{G}-Q\left(H D_{G}\right)_{x}}{Q\left(H D_{G}\right)_{y}-Q\left(H D_{G}\right)_{x}}, & \text { otherwise, }\end{cases} \\
C S_{I}= \begin{cases}0, & H D_{I}>Q\left(H D_{I}\right)_{1-x}, \\
-\frac{Q\left(H D_{I}\right)_{1-x}-H D_{I}}{Q\left(H D_{I}\right)_{1-x}-Q\left(H D_{I}\right)_{1-y}}, & \text { otherwise, }\end{cases}
\end{gathered}
$$

where $Q\left(H D_{G}\right)_{x}$ and $Q\left(H D_{I}\right)_{y}$ are the quantile points at the quantile $x$ and $y$ for genuine and imposter scores, respectively. The levels of the quantiles were optimized empirically. The values resulting in significantly improved verification performance are $x=0.7$ and $y=0.9$. Fig. 7.1 illustrates genuine and imposter distributions typical for HDs. 
The CS of biometric samples is predicted using a nonlinear adaptive mapping and vectors of quality measures (input parameters). The nonlinear adaptive mapping is trained using a set of labeled data in the form of vectors of quality measures and the corresponding CS values obtained using equations (7.3) and (7.4). At the testing stage the CS value is predicted based on vectors of quality measures only. This information is used to keep or discard the corresponding matching score in order to improve the performance of the original matcher. The procedure of predicting the CS of matching scores between two biometric samples $A$

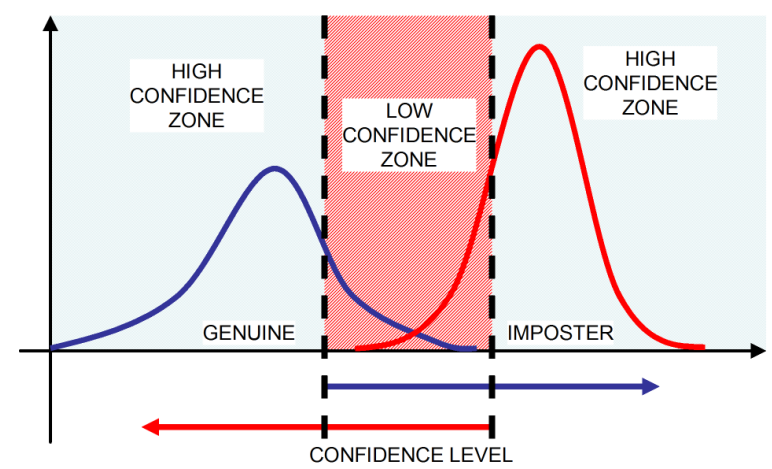

Figure 7.1: Illustration of the Confidence in Scores (CS).

and $B$ is $C S_{A B}=\hat{f}_{C S}\left(\mathbf{Q}_{A}, \mathbf{Q}_{B}\right)$, where $\hat{f}_{C S}(\cdot)$ is a multivariate adaptive mapping (FFNN in our case) estimated using training data.

\subsubsection{Quality Sample and Template Features (QST)}

The third method suggests to treat quality vectors as weak features that can be combined with biometric template features. Let $\mathbf{Q}_{A}$ and $\mathbf{C}_{A}$ be a vector of $K$ quality measures and a template vector associated with a biometric sample $A$. Then the extended template, denote it by $\mathbf{F}_{A}$, of the sample $A$ is the vector $\mathbf{F}_{A}=\left[\mathbf{Q}_{A}^{T}, \mathbf{C}_{A}^{T}\right]^{T}$.

When two biometric samples $A$ and $B$ are compared, the distance (or similarity) between $\mathbf{C}_{A}$ and $\mathbf{C}_{B}$ will be saved as a matching score $M S_{A B}$, while the quality vectors $\mathbf{Q}_{A}$ and $\mathbf{Q}_{B}$ will be treated as extra dimensions that may improve performance of the original matcher, provided these dimensions contain sufficient discriminative information. As shown in Fig. 7.2 the input to the high dimensional classifier is a vector $\left[\mathbf{Q}_{A}^{T}, \mathbf{Q}_{B}^{T}, M S_{A B}\right]^{T}$. The output of the classifier is a decision made by the classifier. The decision is a binary valued variable 


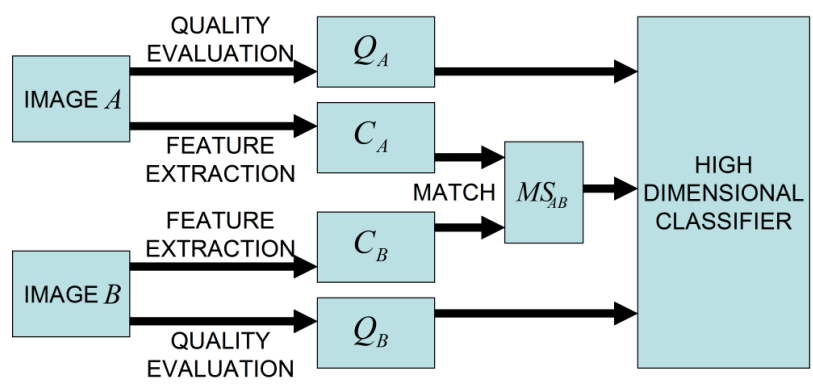

Figure 7.2: Combining quality vectors and templates.

corresponding to $\{$ Genuine, Imposter $\}$. The high dimensional classifier is implemented using a nonlinear adaptive mapping. Denote $\eta_{A, B}$ as the output variable predicted using vectors of the quality measures and the matching score of the biometric samples $A$ and $B$. Then the prediction procedure is described as $\eta_{A, B}=\hat{f}_{Q S T}\left(\mathbf{Q}_{A}^{T}, \mathbf{Q}_{B}^{T}, M S_{A B}\right)$, where $\hat{f}_{Q S T}$ is an estimated version of $f_{Q S T}$.

\subsection{Experimental Results}

The results illustrate the feasibility and utility of our enhanced biometric matchers for iris biometric. We briefly describe the data and experiments below.

All experiments were performed using ICE 2005 dataset [74]. The matching procedures followed a modified log-Gabor implementation by Masek [41] refined by the authors. 26, 867 genuine matching scores and 4,331,761 imposter matching scores from 2, 953 samples were generated.

\subsubsection{Neural Network}

The nonlinear mapping is implemented using Feed Forward Neural Network (FFNN). Training data are assigned labels according to the functional use of the mapping and a set of input and output parameters. The final design is achieved by trading off the complexity and the performance of the network with two hidden layers. For the iris experiments, the first hidden layer of the FFNN is composed of 16 neurons while the second layer is composed of 2 neurons. The training data are divided randomly in two subsets: a learning subset composed of $60 \%$ of training data and a validation subset made of remaining $40 \%$ of data. 
The training process stops when the mean square error drops below $10^{-4}$. The experimental results described below are obtained using codes from the Neural Network Toolbox in $M A T L A B^{T M}$.

\subsubsection{QS evaluation}

The performance of the QS method is evaluated by randomly selecting 1, 500 iris images from the ICE 2005 dataset to form the training set. The remaining 1,453 iris images are used to form the testing set. The $Q S$ of unlabeled images from the testing set is predicted based on the quality vector (quality factors 1 through 10) and by using a FFNN trained on labeled samples. During performance evaluation, unlabeled images with the value of predicted $Q S$ above a preset quantile are retained. Fig. 7.3 displays three Receiver Operating Characteristic (ROC) curves parameterized by zero, $10 \%$ and $40 \%$ quantile levels. The ROC curve marked as "original" is parameterized by zero quantile level, which means that no poor quality biometric samples were discarded. Note that by discarding only $10 \%$ of iris images with the low predicted $Q S$ index, a considerable performance improvement can be achieved. Fig. 7.4 displays a box plot of the Equal Error Rate (EER) as a function of the quantile used to select iris samples with high $Q S$ value. It is a summary of ten independent trials, where training and testing data are sampled at random. It can be observed that regardless of the composition of training and testing data, removing iris images characterized by low predicted $Q S$ improves matching performance of the original matcher. The higher the value of $Q S$, the better the performance is.

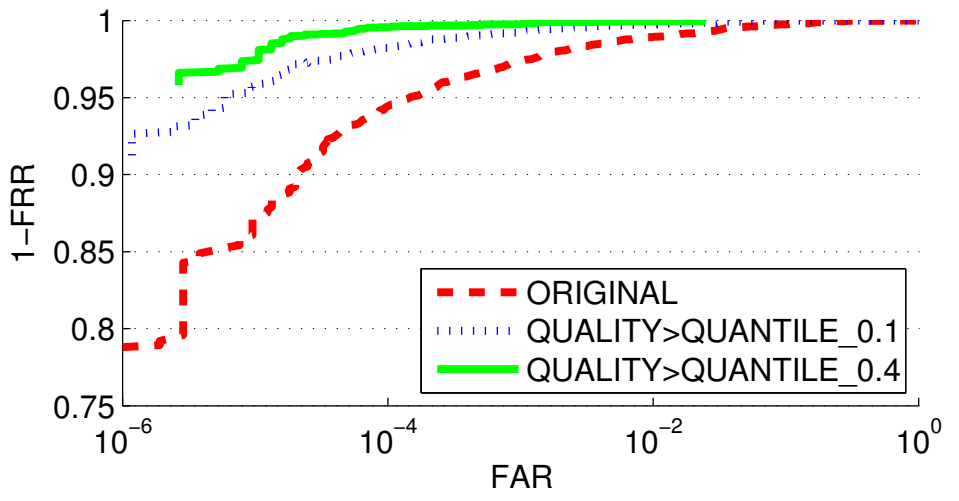

Figure 7.3: Performance improvement achieved by selecting only images with high QS values. 


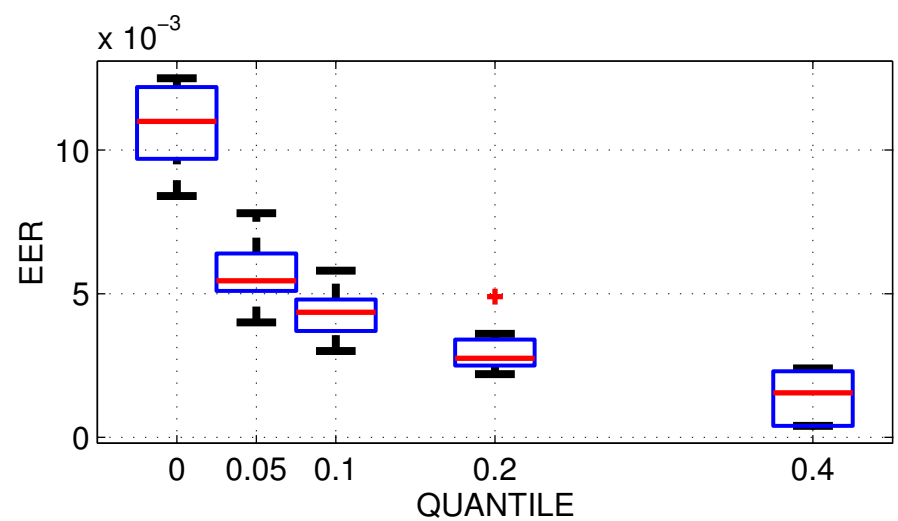

Figure 7.4: Performance improvement by selecting only images with a quality value larger than a certain quantile.

\subsubsection{CS Evaluation}

To assess the performance of the CS method, 20,000 genuine matching scores, 200, 000 matching imposter scores and the vectors of quality measures associated with iris images were used to train a FFNN. The remaining data were used for testing. The matcher was designed to be symmetric with respect to quality vectors, that is, if $\mathbf{Q}_{A}$ and $\mathbf{Q}_{B}$ are two vectors of quality measures associated with iris image $A$ and $B$, training included both the pair $\left(\mathbf{Q}_{A}, \mathbf{Q}_{B}\right)$ and the pair $\left(\mathbf{Q}_{B}, \mathbf{Q}_{A}\right)$ and the associated matching score. The testing experiment is similar to the experiment of the previous subsection with the difference that pairs of quality vectors are used to predict the $C S$ values.

Fig. 7.5 shows three ROC curves: the original curve, the curve formed from iris data with the predicted CS values exceeding $20 \%$ quantile and the curve formed from iris data with the predicted CS values exceeding 50\% quantile. Performance improves when low confidence matching scores are discarded. Fig. 7.6 summarizes the results of ten trials. Again, training set is formed by randomly sampling iris images from a larger set. The trends and results are consistent.

\subsubsection{QST Evaluation}

The matcher is now a FFNN trained and tested as follows. During training the label "1" is assigned to all genuine vectors on the input and the label "-1" is assigned to all imposter vectors on the input to the neural network. During testing the output label is predicted 


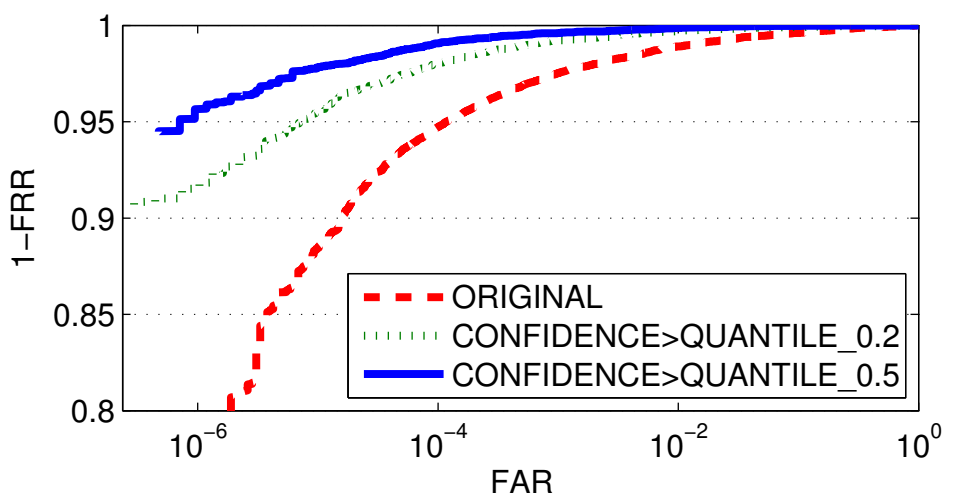

Figure 7.5: Performance improvement achieved by selecting only matching scores with high CS values.

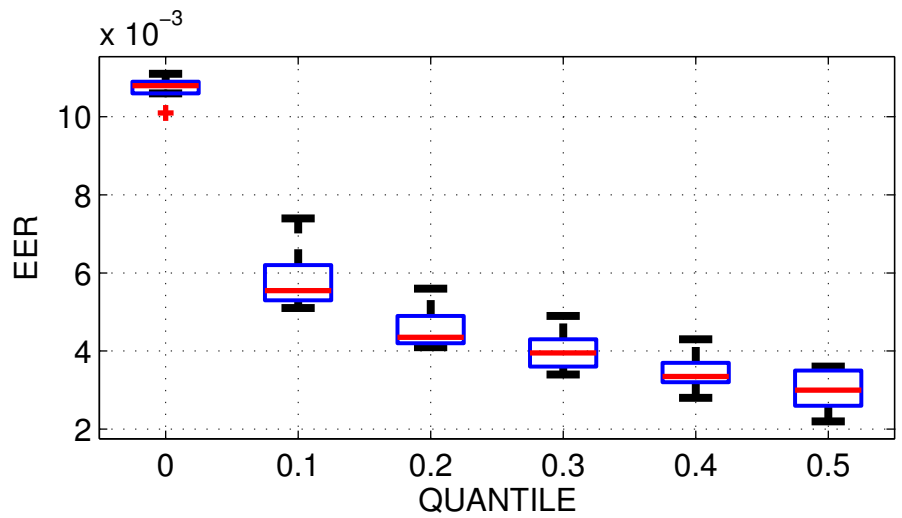

Figure 7.6: Performance improvement by selecting only matching scores with a confidence level higher than a certain quantile.

based on the input vector of quality metrics and the original matching score. The output label in this case is a real number. The high dimensional classifier makes decision in favor of Genuine class if the output label is close to 1. It decides in favor of Imposter, if the output label is closer to -1 . When the decision threshold varies, the performance of the high dimensional classifier is characterized by the ROC curve.

To assess the performance of the QST method 20,000 genuine scores and 200,000 imposter scores and associated quality vectors were involved in training. The remaining vectortriplets were used for testing. The success of the QST method depends on the data selected for training and testing. A single trial out of set of 20 trials resulted in a perfect separation of genuine and imposter matching scores. In the other cases the $\%$ improvement was between $20 \%$ and $35 \%$. The results of first five trials are shown in Table 7.1. In columns 2 and 3 it displays the values of $E E R$ without/with quality factors. In columns 4 and 5 the table 
displays the values of False Reject Rate (FRR) evaluated at 0.001 False Accept Rate (FAR).

Table 7.1: Performance with/without quality factors

\begin{tabular}{|c|c|c|c|c|}
\hline & $\begin{array}{c}\text { Original } \\
\text { EER }\end{array}$ & $\begin{array}{c}\text { New } \\
\text { EER }\end{array}$ & $\begin{array}{c}\text { Original FRR } \\
\text { at 0.001 FAR }\end{array}$ & $\begin{array}{c}\text { New FRR } \\
\text { at 0.001 FAR }\end{array}$ \\
\hline Trial 1 & 0.0107 & 0.0065 & 0.0251 & 0.0197 \\
\hline Trial 2 & 0.0110 & 0.0076 & 0.0246 & 0.0186 \\
\hline Trial 3 & 0.0109 & 0.0072 & 0.0251 & 0.0192 \\
\hline Trial 4 & 0.0109 & 0.0068 & 0.0249 & 0.0193 \\
\hline Trial 5 & 0.0106 & 0.0086 & 0.0244 & 0.0229 \\
\hline
\end{tabular}

\subsection{Conclusions}

Three new methods for matching iris biometrics using quality metrics are proposed. The methods are adaptive and use nonlinear mappings for making predictions on quality of the sample, confidence of the matching score and classification result of the combined quality-template feature set. The experimental results reported illustrate the importance of predictive and selective integration of quality measures for biometric authentication and show significant advantages compared to existing methods. Our future research will focus on (i) designing and analyzing quality based multi-modal fusion schemes; and (ii) developing the concept of quality at different processing levels in biometric systems. 


\section{Chapter 8}

\section{Future Directions and Preliminary Work}

While in the literature iris recognition systems are presented as complete systems (from data collection to performance validation), practical applications require additional substantial tuning and adaption. In this chapter, we illustrate this with two examples. Two applications will be studied and preliminary results will be reported.

\subsection{Active Iris Recognition}

In this section, a concept of active iris recognition is introduced and its feasibility is demonstrated. By involving an industrial robotic arm in the process of iris image acquisition, the iris capture device will have in total $6+$ degrees of freedom to deal with moving subjects at a distance. The subject will be in focus for most of time as the capture volume is increased. Therefore high quality iris images are ensured. This concept can be adopted to applications such as airport surveillance or security check point.

\subsubsection{Introduction}

Iris recognition from a distance and in unconstrained environment is a challenging problem. To ensure a sufficient resolution of an iris image, a high zoom lens must be used. The main limitation of a high zoom lens is its small capture volume. The view angle is narrow and the depth-of-field (in-focus range) is small. The object can quickly get out of view or 
out of focus. Motion blur can also be observed in these images.

Two companies have recently provided solutions to this complex problem: Sarnoff [6] and GE [120]. Sarnoff uses a booth to keep the person in the view horizontally. Two or more cameras are vertically aligned together to make the system suitable for different people with different heights. Multiple shots are taken to make sure that at least one in-focus image is acquired. GE uses stereo imaging to find the position of the target. As soon as the system locates the head, a PZT camera with IR illumination will track iris area and acquire iris images.

Both solutions are robust and reliable solutions. However, there is always room for improvement. For Sarnoff system, only one user can pass the booth at a time, even with multiple cameras. A person who wants to pass needs to cooperate to ensure reliable identification. For the GE system, a person also needs to cooperate to ensure that the PTZ camera is in the required position and is able to capture the iris. The PZT camera normally has a small aperture, which is not suitable to capture clear images if the experimental environment is not ideal. The ability to process two or more users at the same time is also limited.

The main idea of this R\&D is to add extra degrees of freedom to the system. Therefore the throughput of the system is expected to increase together with the quality of acquired iris images. Those extra degrees of freedom are introduced by involving a robot in the system. A high-end iris capture camera, which has a large aperture but a relatively small depth-of-field, will be mounted on the tip of the robotic arm. The adaptively controlled movements of the robotic arm will increase the capture volume of the system and will also compensate for the distortions due to the relative motion introduced by the moving target. The motion blur and the defocus blur are expected to be reduced to minimum. This system also puts minimal constraints on users. A mother can hold hands of her children while they are passing the hallway. They will keep moving and following the signs, and at the same time their iris information will be captured. 


\subsubsection{Scenarios}

The implementation depends on an application, such as surveillance at airports or iris recognition for the people in the car at the security check point. The hardware setup for these two scenarios will be explained in detail. However, similar setups can be used for other applications.

\section{Airport Surveillance}

The setup for this application is not very complex. A stereo camera, camera network or a 3D scanner will be mounted on the ceiling to search for the possible subjects, walking or standing individuals. Each detected subject will be marked for iris capture. Two or more 7-joint robotic arms will be placed side by side to form a cooperative pair or team, respectively. The number of robotic arms will be equal to the number of subjects the system can track. Each robotic arm will be equipped with a high zoom iris capture camera equipped with IR illumination source. The focal length of the lens will be fixed. Each robotic arm will follow its subject (the head of the user, for example). The camera will always be positioned such that it faces the subject from the same distance. The subject will be always in focus as it is inside of enlarged capture volume. Because the camera will move at the same speed as the subject moves, the motion blur will be reduced to the minimum level.

To make the capture more user-friendly, a proper layout and equipment setup are required. An L-shaped corner is preferred. Cameras will be placed behind a one-way pass glasses. There will be information signs (sounds can be used too) on the wall to attract the view of the users. An illustration is provided in Fig. 8.1. The gray color box inside of the figure shows the capture volume without using the robotic arm. And the larger blue box marks the increased capture volume due to use of robotic arms.

\section{Iris Recognition at a Security Check Point}

To use the iris recognition technology at a security check point, such as the gate entrance of a high security military facility during rush hours, is challenging, since every passenger 


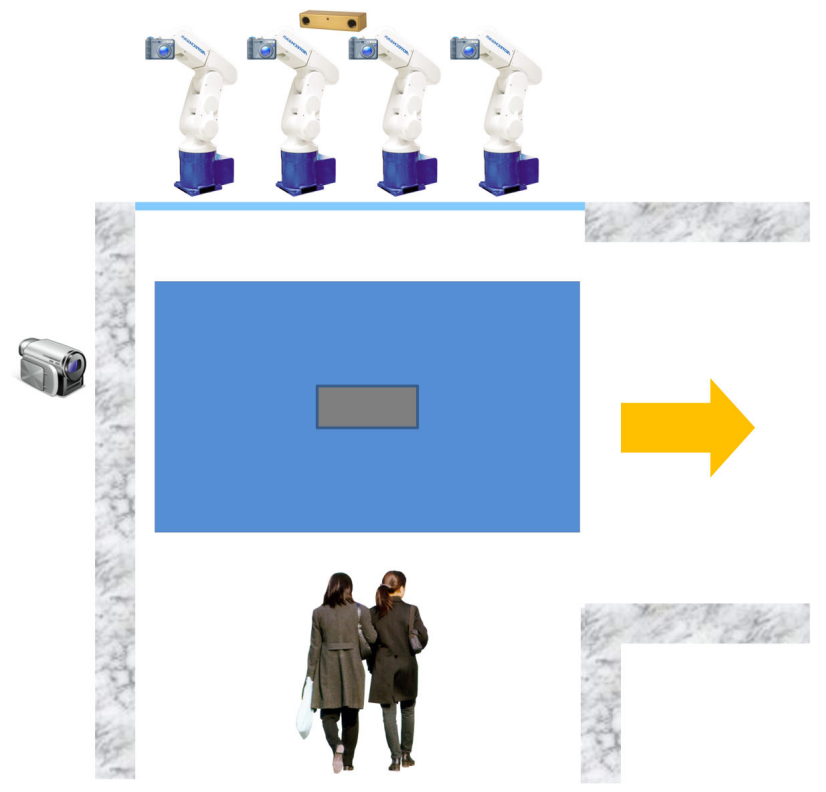

Figure 8.1: The setup at an airport.

would be required to come out for an iris scan. This problem can be solved using active iris recognition, which ensures increased processing throughput. The setup is illustrated in Fig. 8.2. Obviously, this application requires minimum subject's cooperation.

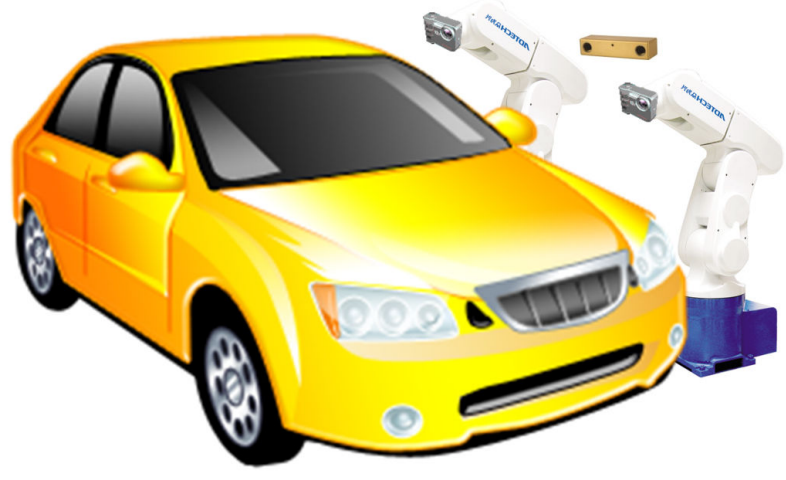

Figure 8.2: A potential setup at a security check point.

\subsubsection{Methodology}

In this section, only the first scenario will be discussed. The second scenario is less complex and its implementation is more straightforward because subjects are expected to 
cooperate.

\section{D Scanner}

The first step is to take a 3D measurement of a subject. There are a lot of existing technologies for extracting 3D information. For example, a 3D laser scanner can be used to acquire a 3D point cloud. Some models also can capture color intensity information at the same time. High speed 3D laser scanner, however, is very expensive. A much cheaper solution is to involve stereo imaging. There are some existing commercial stereo vision products, such as Bumblebee ${ }^{\circledR}$ series from Point Grey [121], which are calibrated at high precision. A single camera solution using structured light is also fast and cheap. However, the strobe light can be very irritating for subjects. It may also damage the collected Near-Infrared (NIR) data.

For current prototype development, we involved Kinect ${ }^{\circledR}$ from Microsoft which is designed for the game console XBOX 360 [122]. Kinect relies on a 3D scan technology called "Light Coding ${ }^{T M}$ " which extracts 3D information with the aid of a continuously-projected NIR structured light [123]. A monochrome CMOS sensor reads the coded light back, and then a processor calculates the depth image of the scene. The functionality of Kinect ${ }^{\circledR}$ is illustrated in Fig. 8.3.

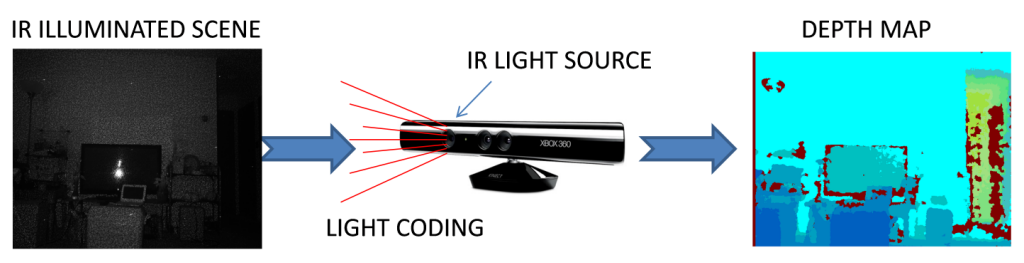

Figure 8.3: The technology used in the Kinect ${ }^{\circledR}$.

\section{Background Estimation}

Background estimation is the first step to track subjects. In our implementation, 3D scanner is mounted on stable platforms. No registration or alignment is necessary between 
frames. 100 frames are used to estimate the background. For a 3D depth map, each pixel intensity value corresponds to a distance measurement in a certain direction. Median values along the time axis for 100 frames are regarded as the "true" distance measurement of the background. The color image of the scene, and the estimated background are provided in Fig. 8.4.
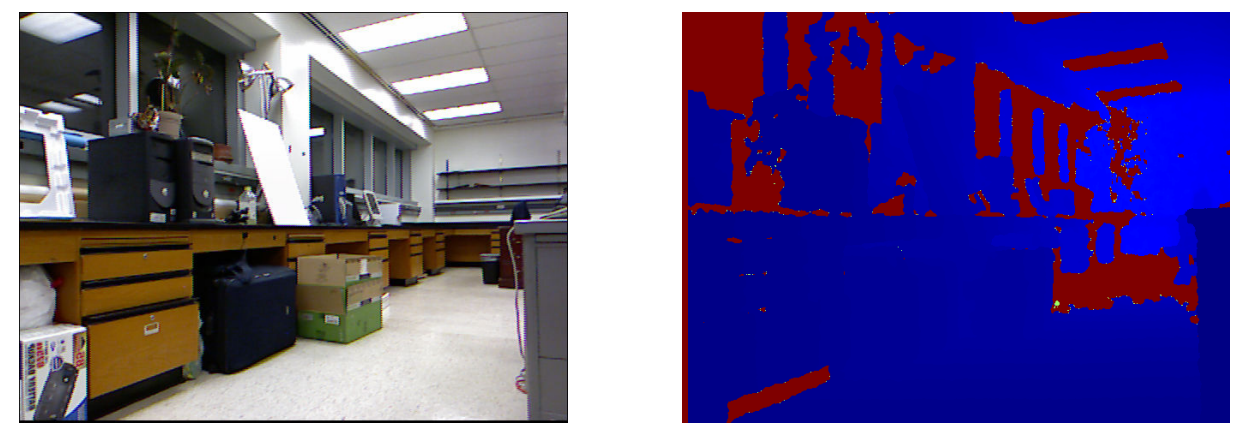

Figure 8.4: The background (a) and its corresponding depth map (b).

\section{Background Subtraction}

The calculated depth map is noisy and so is the foreground mask. To get the clear outline of the subject, the binary foreground mask is further refined: 1) several "erode" morphological operations are applied to remove isolated noisy areas; 2) while true subject areas are also affected, remained "1"s are used as seeds to find the original subject boundary. The procedure is illustrated in Fig. 8.5.

\section{Head Detection}

Single Subject For a standing person, the head is the highest part of the body. And it is one end of vertical body axis: an imaginary longitudinal line through the center of the body. Now, mass center of each foreground subject will be detected. Then from this mass center, all candidates of "head location" will be evaluated based on the height and the horizontal location. The one with the highest score will be treated as the true head. The procedure is illustrated in Fig. 8.6. Once the location of the head is determined on the 2D image plane, 


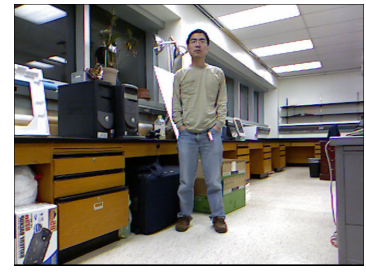

(a)

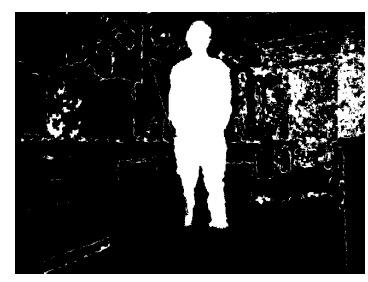

(d)

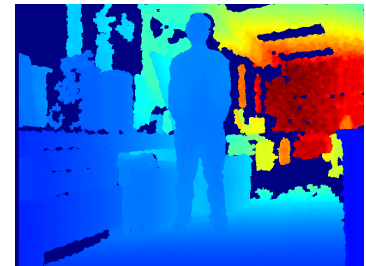

(b)

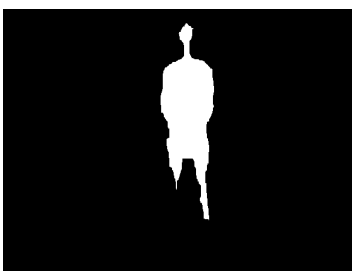

(e)

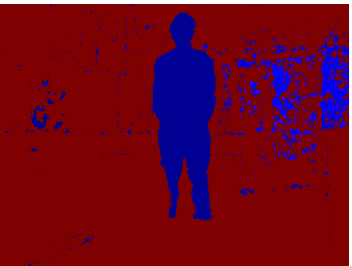

(c)

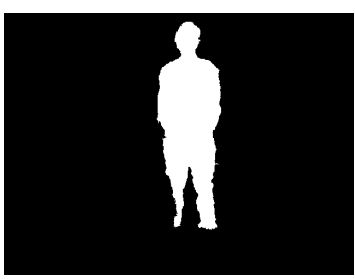

(f)

Figure 8.5: Noise removal: (a) the color scene image, (b) the original depth map, (c) differences from the trained background, (d) the original foreground mask, (e) eroded mask where only parts of true subject are left and (f) the final foreground mask.

the real world coordinates of the head can be calculated based on system parameters of the 3D scanner.

Separable Subjects It is possible that two subjects walk along together. However, in most cases, they are still separable. The weak (narrow) connections between them can be easily detected. Based on the experiment, the length of 200 pixels was selected as the threshold to identify narrow connections. When a column has less foreground pixels than the threshold, the whole vertical line will be cleared. Once subjects are separated at the weak connection point, they will be treated as different subjects by the algorithm. Then the head location will be detected separately for each subject using the algorithm introduced in 8.1.3. The foreground mask before and after the separation are illustrated in Fig. 8.7 (c) and $(\mathrm{d})$.

Overlaps It is possible that one subject is heavily occluded by others. They are not separable in the 2D binary foreground mask because the overlapped area is thicker than the cutting threshold. However, in this case, the distance difference between those two subjects must be thicker than a body and is obvious enough to be detected. The outline of subjects 


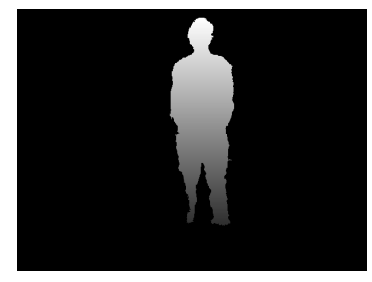

(a)

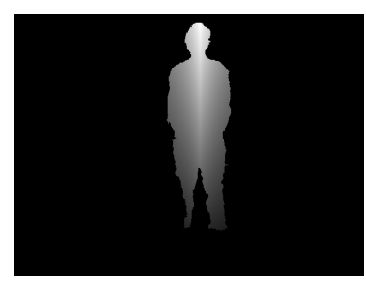

(d)

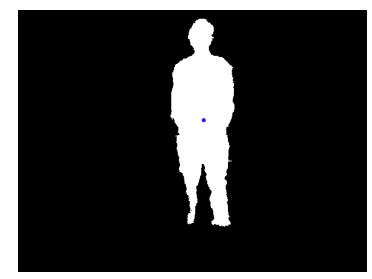

(b)

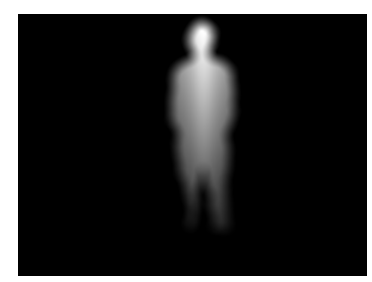

(e)

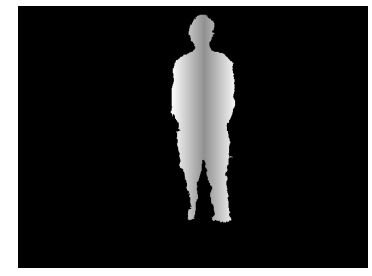

(c)

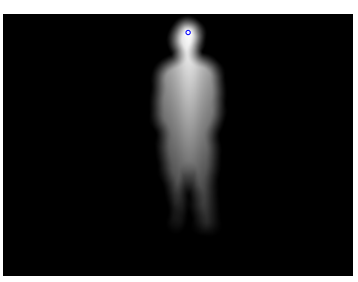

(f)

Figure 8.6: Head detection: (a) the initial score based on the height, (b) the center of mass, (c) the penalty for the off-center, (d) the combination of (a) and (c), (e) the penalty for the edges and (f) the final head(forehead) candidate.

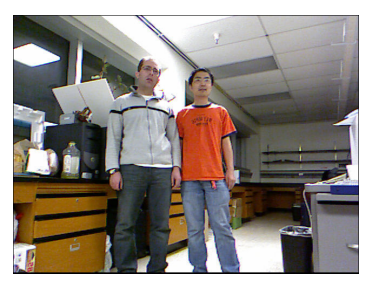

(a)

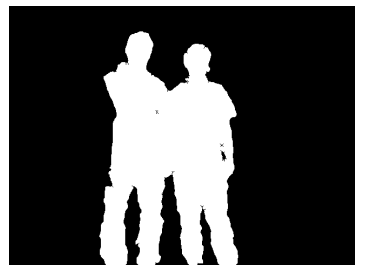

(c)

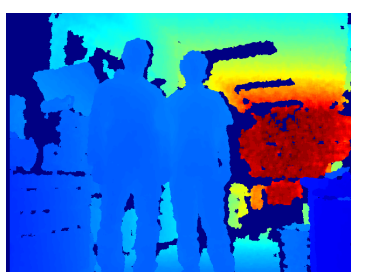

(b)

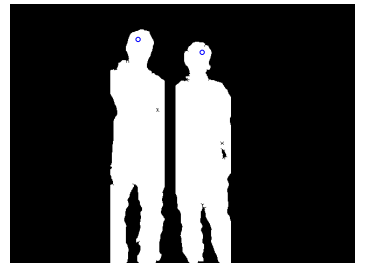

(d)

Figure 8.7: Head detection when there are connections: (a) the color scene image, (b) the original depth map, (c) the narrow connection between two subjects and (d) separating subjects using vertical cuts. 


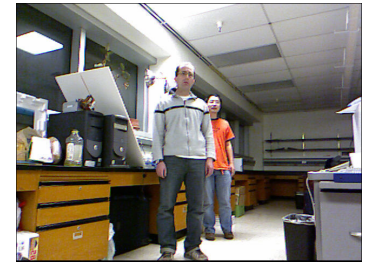

(a)

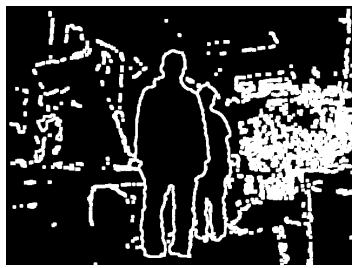

(d)

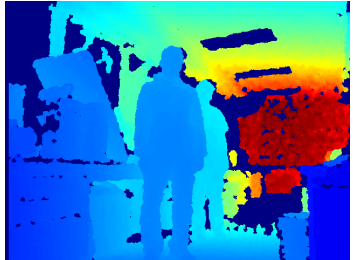

(b)

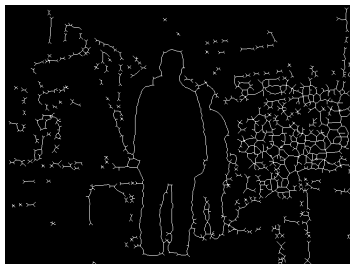

(e)

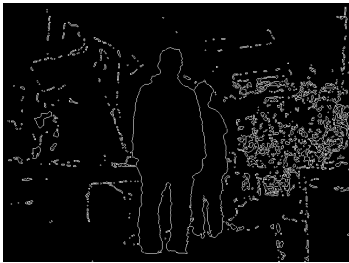

(c)

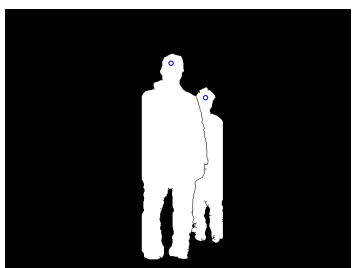

(f)

Figure 8.8: Head detection when there are overlaps: (a) the color scene image, (b) the original depth map, (c) all clear distance changes in the scene, (d) the expanded selection, (e) the "skeleton" and (f) the final successful head detection result.

can be estimated using "edge" detection algorithms on the depth map. While those edges may not be continuous because of noise (Fig. 8.7 (c)). The binary edge mask is expanded to make separated edges connected to each other. However, it is now too thick to be used. Instead of expanded edge mask (Fig. $8.7(\mathrm{~d})$ ), the "skeleton" of it (Fig. $8.7(\mathrm{e})$ ) is used. The final detection result is provided in (Fig. 8.7 (f)).

Face Detection For more complex situations, face detection can be used to ensure that all heads will be detected. Just like how Kinect is build, a color camera can be aligned with the 3D scanner. With the color image sequence, face detection algorithms can be used to detect the location of the head. To extract the 3D location of the head, the color camera can be perfectly aligned with the 3D scanner to make the registration between the color view and the depth map straightforward. A public available face detection code is tested on the color image captured by the Kinect [124]. The face detection result is presented in Fig. 8.9 where face candidates are marked out using green boxes. The false alarm (the wrong candidate) in this image can be easily eliminated using the foreground mask extracted from the depth map in Sec. 8.1.3. 


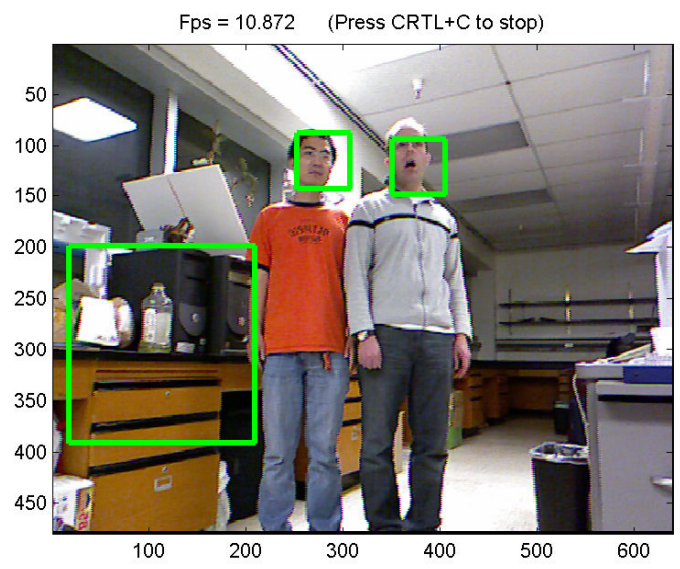

Figure 8.9: The face detection result.

\section{Model for the Robotic Arm}

A 6-link Puma robot model and corresponding control algorithm available online are used for the demonstration of the proposed concept [125]. In real implementations, if a high zoom NIR camera needs to be mounted at the end of the robotic arm for the iris data collection, then an extra degree of freedom needs to be added at the end of the arm for the camera angle adjustment. Not just Puma, most other 6 or 7 joints industrial robots with an advanced controller can fulfil requirements of active iris recognition applications.

\section{Control of the Robot}

All robotic arms have to finish their own tracking task based on the real world coordinates calculated by the 3D scanner. While multiple independent robotic arms attempt to perform concurrent operations in a limited working space, advanced collision avoidance algorithms, such as dynamic interference zones, are needed to protect robotic arms.

The data collection site is well designed to make sure the subject faces the camera most of the time. As the distance between the camera and the subject is relatively long for the size of the robot, all what robot can do is to keep the relative position between the camera and the head same. The compensation of the head pitch or yaw is impossible when the subject is far away because the robot is fixed to the base. Two irises of the same subject will be captured at the same time. 
To make sure the robot can follow the movement of the head smoothly, the moving speed of the head needs to be calculated and predicted. This compensation can reduce the motion blur to the minimum level in the captured iris image.

\section{Live Demo}

The live demo is a simple implementation of active iris recognition. As illustrated in Fig. 8.10, in this demo, a Kinect ${ }^{\circledR}$ will be used to capture the 3D scene depth video. The 3D depth map will be processed using MATLAB to extract the subject. The $\mathrm{x}, \mathrm{y}, \mathrm{z}$ coordinate information of the head will be further detected and sent to the robot control subsystem for a motion planning. The robotic arm will then start to execute the actions according to the plan and follow the move of the head. A snapshot of the interface is provided in Fig. 8.11 where blue dots indicates the trajectory of the camera.

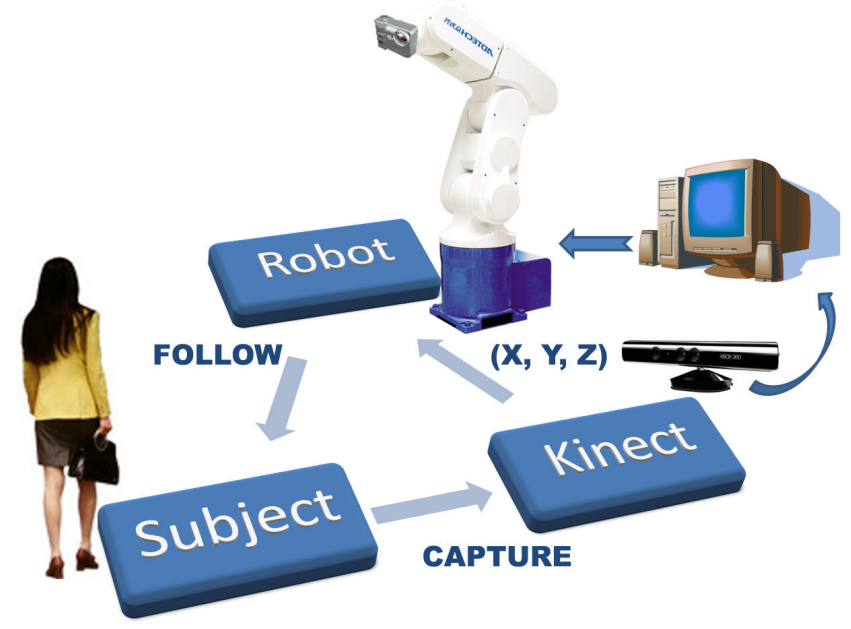

Figure 8.10: The diagram of the demo.

\subsubsection{Discussions and Future Work}

The main concept here is using a robot to follow the subject for high quality image acquisition. Kinect is just one of many methods to track the person. Compared with PZT (Pan Zoom Tilt) cameras, for a short distance, the camera at the end of robot arm not only can keep the subject in focus, but also keep the subject in front-view position in most of 


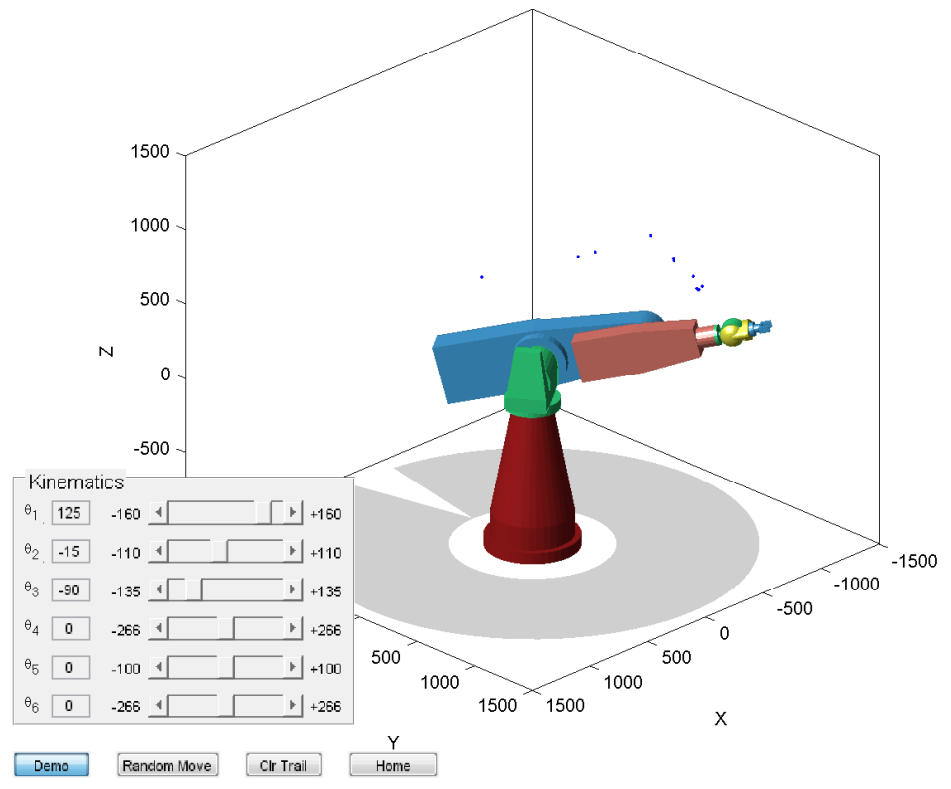

Figure 8.11: The robotic arm is following the position of the head in the live demo.

time. The cost is high, but is still reasonable for the benefit the system can achieve. As the concern of the vibration, it is true that moving cameras will not be suitable for long distance image capturing. This project is designed for a working distance of 1.5-3 Meters. While the subject is closer, under the same illumination condition, a faster shutter speed can be used. Furthermore, a heavy robot and a light camera will be used to reduce the level of the sway. All those assumptions from the preliminary result will be verified in the next stage when the real robot arm is used.

It is proposed that an active iris recognition system can be implemented at a reasonable cost with a gain of better quality images than existing "iris on the move" implementations. In the future, a more complex prototype system with real robots will be built for a real scenario demonstration. 


\subsection{Social Connections for Improved Performance of Biometric Systems}

For security applications and for recognition in close proximity, iris biometric is the most promising biometric technology demonstrating extremely high recognition capabilities. However, its coverage and scalability is limited resulting in inability to cover billions of people. A traditional approach to tackle this problem is to involve a multi-biometric system. An alternative and also complimentary approach to involving multiple modalities for very large population coverage is to take into account social connections of involved individuals. In this section, social connections for improved performance of biometric systems are discussed in both identification and verification scenarios. Experimental results involving novel models and applications demonstrate substantial performance improvements.

\subsubsection{Introduction}

Iris biometric is the most promising biometric technology for security and personal applications due to its extremely high recognition capabilities. However, it is still not good enough to cover billions of people. One of possible solutions proposed in the literature in the past is multibiometrics [126] [127]. It can improve the performance of biometric systems and therefore increase the population coverage.

Multibiometric systems need multiple sources, and they can be multi-sensor, multialgorithm, multi-instance, multi-sample, multimodal and hybrid [127]. Multiple sources can be fused at five different levels: sensor, feature, score, rank and decision [128]. However, the total complexity of the system and overall computational and processing cost will be high, and it is not easy to expand the existing unibiometric system to a multibiometric system.

An alternative and also complimentary approach to involving multiple instances, even with multiple modalities or hybrid for very large population coverage, is to take into account a social network. For example, local police officers never have problems to identify or verify a local person because of a local social network, which they take into account to recognize a person. A small scale network is illustrated in Fig. 8.12. If two or more trustworthy persons or witnesses provide the same information that can be used to identify a suspect, then it 


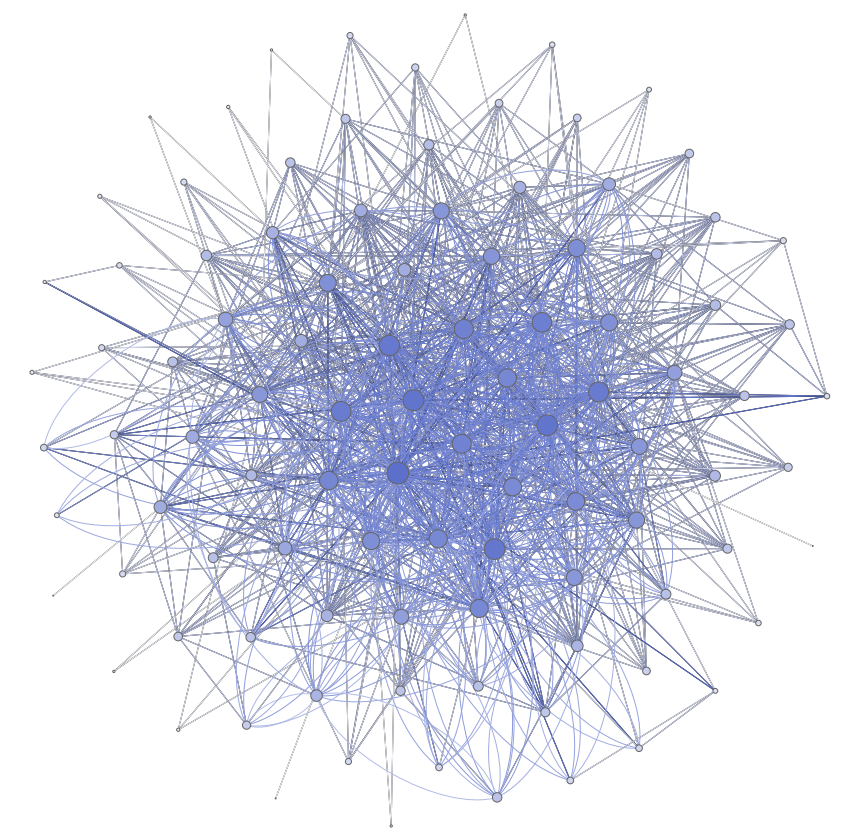

Figure 8.12: The visualization of a small social network.

will be highly probable that the suspect is the person they believe he is.

Another example where local social network could be useful is the Department of Motor Vehicles (DMV). When a local resident applies for a driver license, he/she has to provide a proof of residency. If he/she fails to do this because he/she just shares apartment with another person, and the other person paid all bills in the past 6 months, then he/she can ask his/her roommate for a referral to prove the residency. Actually, in some cases, social network itself can identify individuals [129]. In this work, no manual (subject-based) identification is performed. However, the idea how DMV uses the local social network will be adopted.

The most similar work exist is "Facial Recognition With Social Network Aiding" from Google [130]. When they try to identify a person in a picture, they select the rank 1 candidate based on not only facial recognition metrics of visual similarity but also the social connection metrics. Basically, information obtained from other pictures of the identified person, will help to identify persons in an image query. In this work, both identification and verification scenarios are considered. In addition, performance is evaluated using a large scale realistic dataset. Note that the idea introduced in this chapter is not limited to iris biometric only. It also can be applied to face, fingerprint, and other biometric modalities. 


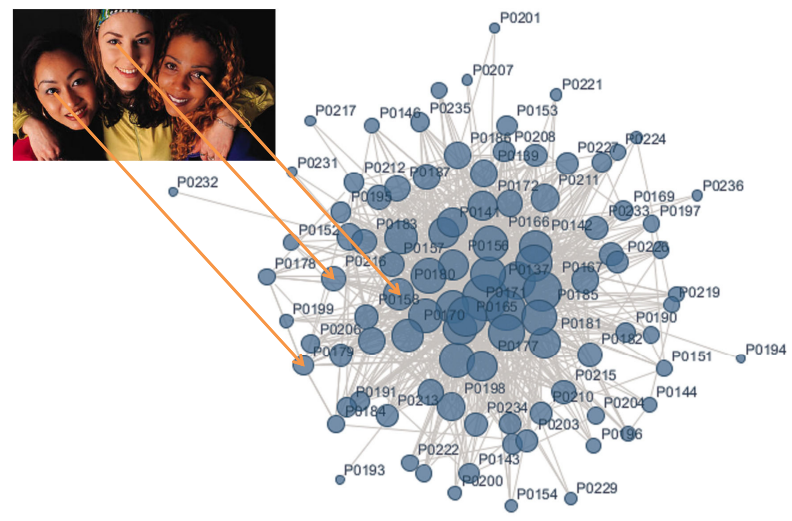

Figure 8.13: The social network match will support the results of biometric identification.

\section{Identification}

In Europe iris biometric is used for an accelerated check in of the passengers at the airports. Iris surveillance application can be easily added for searching suspects or fugitives. This databases with millions of iris images are viewed as large databases. The performance of involved identification algorithms may drop if the size of the database continues increasing. Normally once a threshold is set, only individuals who passed that threshold will be considered as candidates for an unknown identity. If we have a social network map, we may have verified connections between passengers. Just like how Google patent uses social connections, we can reduce the number of candidates further down because the passengers who fly on the same day or take the same air plane are possibly related. The rough idea is illustrated in Fig. 8.13.

\section{Verification}

To explain how a local social network can be used for a biometric verification application, let's consider procedure of creating a account at a bank. A person $A$ wants to apply for a credit card, and the bank wants to verify the identity of the person. Based on the personal information which $A$ provides, the bank extracts the biometric information and makes a comparison. However the single verification result may not be reliable due to a low quality of the query image or due to the presence of many similar patterns in the database. If the iris image is captured at the client's home via a web camera, poor or unconstrained image 
capturing environment may result in a false negative. Even with a constrained capture environment, for a large population application, a high matching score can not represent a genuine match since there may be too many similar irises in the database. For example, a lot of Asian people have clear distinct iris pattern around the pupil area.The remaining 80 percent of iris area is covered by a flat low frequency pattern. This flat area has low uniqueness, and influences the False Accept Rate. Therefore, for this type of irises, even with a pretty high matching score, there is still a high possibility that it is an imposter. Then a potential solution, just like it is used by DMV, is to ask for referrals. Individual $A$ can mention individual $B$ to the bank as a referral. $B$ can be one of family members, or one of his best friends. If $A$ can get enough referrals to provide a sufficient support via biometrics verifications, then the credit card application can be processed. Unlike identification scenario, there are no need to save those connections in the database because the social connections are provided by the user or the client. The privacy concern is reduced to minimum.

\subsubsection{Methodology}

All social connection information has to be saved in a database together with biometric information in identification scenario. However it is not necessary in verification scenario. Two kinds of applications are introduced separately in this section.

\section{Identification}

Large scale identification of a person using biometrics can be a challenging problem because there may be too many similar enrolments. Therefore, soft data related to the query biometrics may be very helpful at this point. Information may be in the form of records about location, time, and biometrics which are collected at the same time. If an extra biometric, such as full face or an image of the other eye, is collected from the same person, then a muti-biometric approach can be used for identification. If data are from another person, then the possible social connection between them may be useful.

In a biometric database with records about social connections in it, a number of query entries is submitted to identify a person. The first step of the identification process is to check if there are social connections between those candidates. Initially the candidates for 


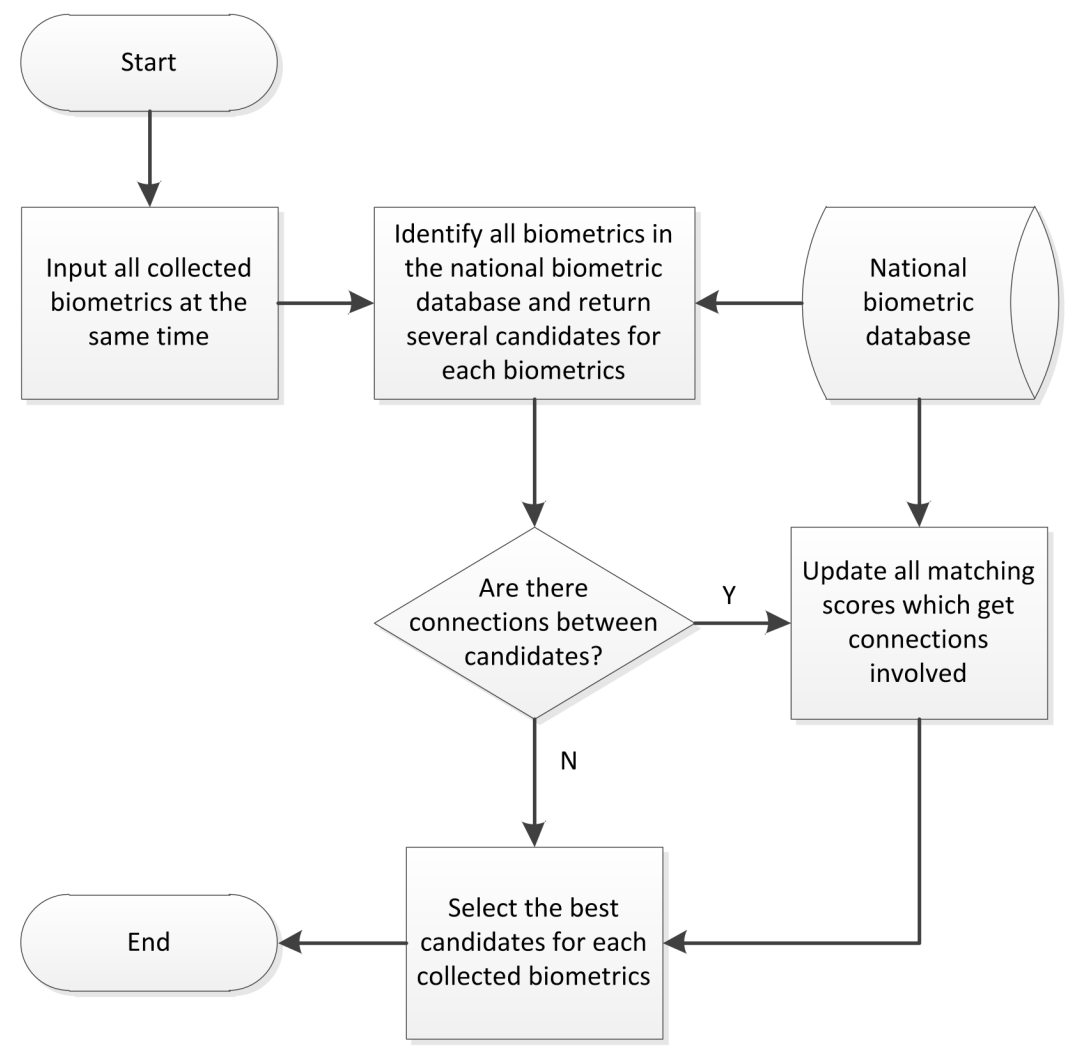

Figure 8.14: The flow chart of a biometric verification system relying on a social network.

each query image will be sorted based on their matching scores, which are used as initial data. A matched social connection from two different query entries will put a little bit more weight to two corresponding candidates. Their ranks in their groups may change because of these extra weights. Multiple connections will give more weights to the related candidates. All matching scores between the query biometrics and the candidates will be updated based on the newly assigned weights. Finally the decision will be made based on the updated scores. Note that this procedure will identify a group of people in place of a single person. The flow chart summarizing the whole identification procedure is provided in Fig. 8.14.

The theoretical motivation behind this approach is that the possibility of an imposter score passing the verification threshold is much lower than those of genuine scores. Then the possibility where a person $A$ 's imposter, which successfully passed the verification threshold, also knows $A$ 's friend $B$ is even smaller. Even more, for a large population, the possibility where two "good" imposters know each other is negligible. 


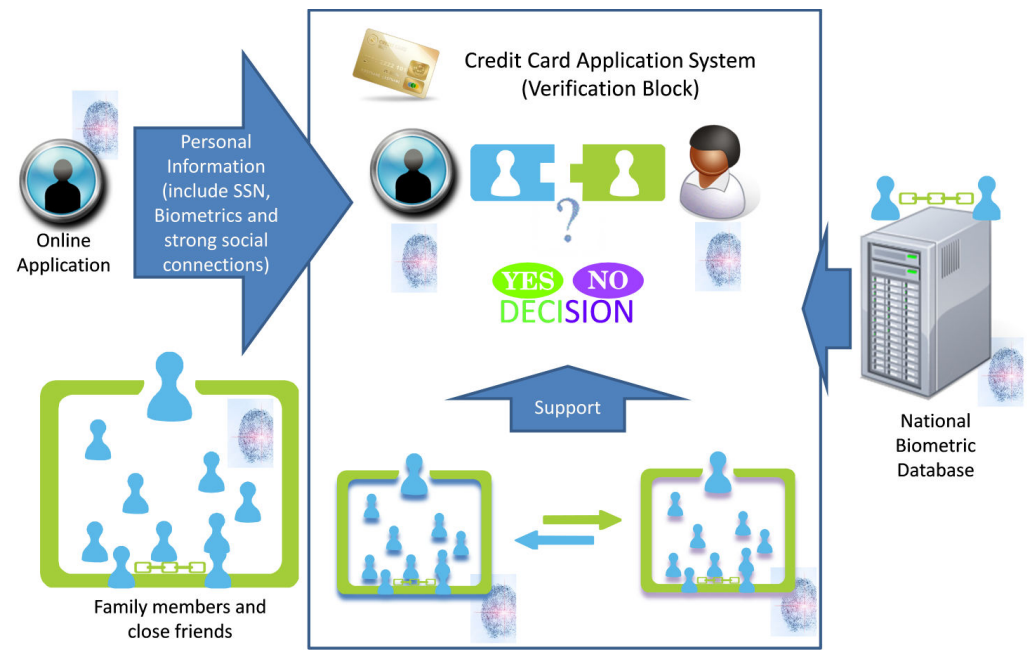

Figure 8.15: The credit card application procedure.

\section{Verification}

When a user attempts to get verified using the expanded biometric system described in Sec. 8.2.1, an assistance and support may be needed from his family members or friends if his/her biometric features are not strong enough. Just as shown in Fig. 8.15, his/her support group will provide their personal ID and biometric information to support the applicant.

The support from family members and friends will result in an improved matching score in the extended biometric system. The performance, uniqueness and the social "credit score" will determine how much support each person can give. A flow chart outlining this idea is presented in Fig. 8.16.

\subsubsection{Experiments}

As mentioned earlier, the method introduced in Sec. 8.2.2 is applicable to all biometric modalities, iris used in this chapter is only an example. A large database of synthetic iris codes is generated, and matching scores in the form of Hamming Distance(HD) for the synthetic data are evaluated. 


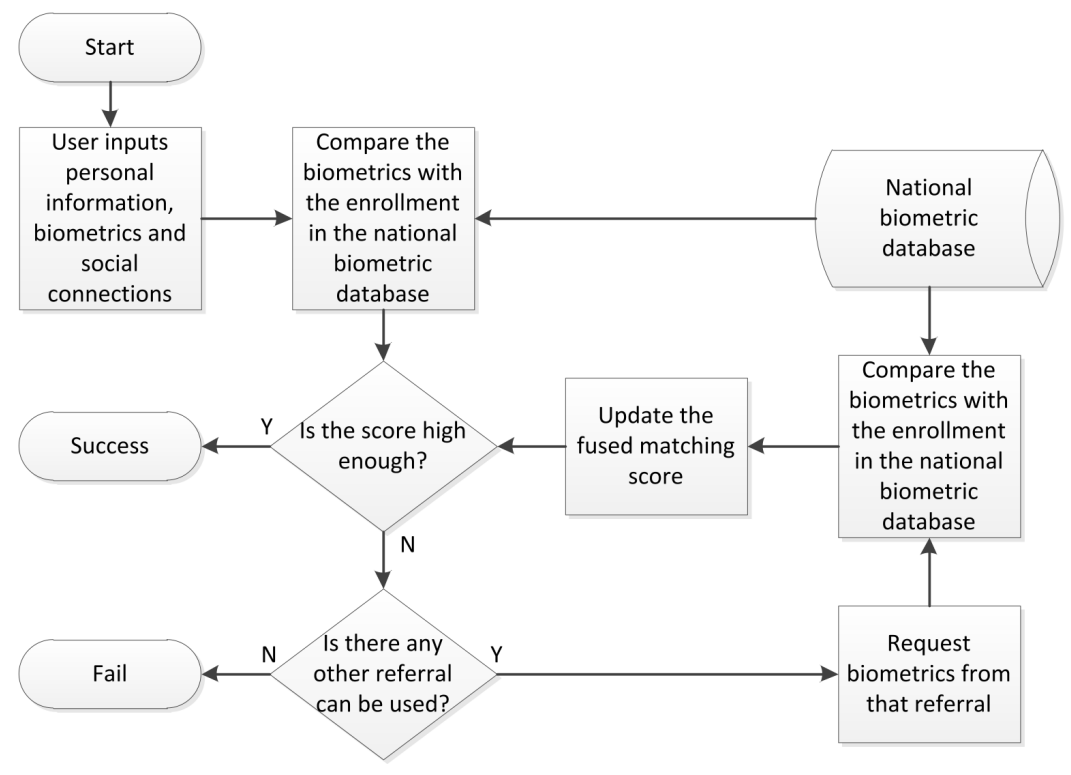

Figure 8.16: The flow chart of a social network assisted biometric verification.

\section{Simulated Data}

Based on the statistic data from Daugman's work [8], a 37000 user database with one enrolled iris code and one query iris code per iris class, was build using Matlab. The iris code has 256 independent bits. The degree of freedom is slightly higher than 249, which is estimated from real data. The variation of genuine score is simulated using Gamma distributed noise. From an original 256 bit iris code, certain number of bits will be flipped to generate a "noisy" code as the enrolment. Query code is generated in the same way. In our experiment, the parameters of gamma distribution are obtained experimentally to achieve the desired genuine distribution. Every query code is compared with every enrolment code. Therefore a $37000 * 37000$ similarity matrix is calculated from this dataset of synthetic iriscodes. Experimental mean and standard deviation of genuine scores are: 0.1131 and 0.0702 . Experimental mean and standard deviation of imposter scores are: 0.4579 and 0.0196 . The single point measure $d^{\prime}$ equals to 6.6874 .

\section{Identification}

For a large dataset, matching query and enrolment images may not result in the first rank matching performance. An imposter image which happens to have a "correct" noise 


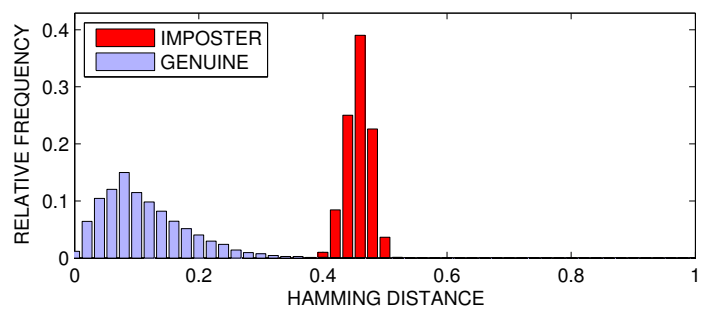

Figure 8.17: The distribution of genuine and imposter scores obtained using 20,000 classes.

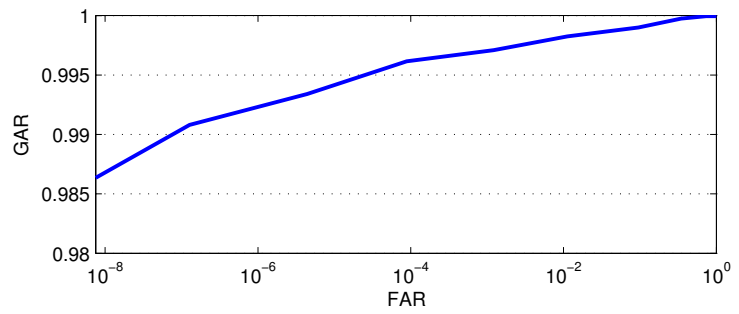

Figure 8.18: The Receiver Operating Characteristic(ROC) curve based on 20,000 classes.

pattern, for example, a heavy occlusion, may result in the rank 1 match. When identities are unknown, there are 3 possible outcomes for a certain verification threshold: 1) no one passed the threshold, 2) only one class passed the threshold, and 3) there are more than one classes passed the threshold. Those 3 cases can be divided further to 7 different cases when identities are discovered. They are illustrated in Fig. 8.19. In case 3, because more than one candidate passed the threshold there will be a ambiguity to solve. To find the true candidate, extra information is needed. When a set of anonymous biometrics collected at the same time need to be identified, it is highly possible that they are connected. The connections are provided or proved by other information sources, such as Federal Bureau of Investigation. With the social network information, a simple solution will be boosting the scores with one or more "connection" matches.

As an example, email communication network from Enron will be used to simulate the network connections [131]. Each node (person) in this network will be assigned a random iris code. Then the performance improvement can be demonstrated by the number of solved ambiguities.

For 36,692 classes, we find that 36,261 classes have genuine scores below the threshold 


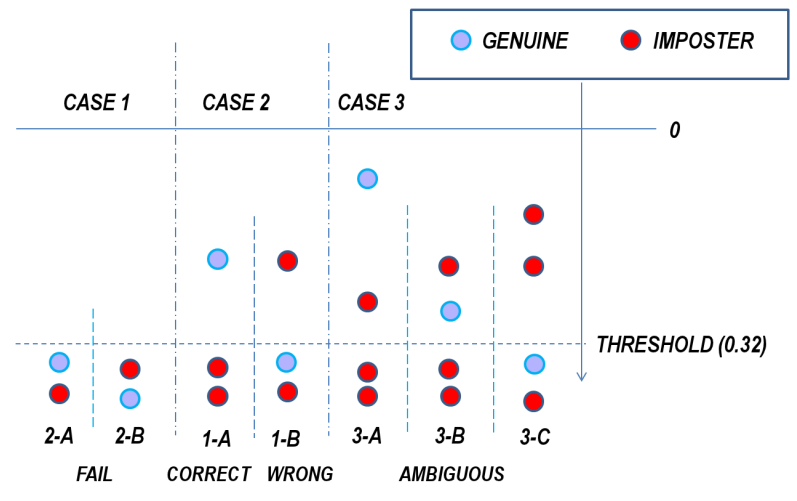

Figure 8.19: The 3 possible outcomes in the identification scenario.

set at 0.32 . Within the set of imposters, only 21 imposter scores exceed the same threshold along with genuine scores. Luckily all passed imposter scores are larger than corresponding genuine ones. However, other than that, totally, 179 classes has a larger genuine score than imposter scores. The performance is summarized in Table 8.1. While the size of the database is increasing, it is possible that at certain point, there are smaller imposter scores than corresponding genuine ones and also smaller than the identification system threshold, such as 0.32 . Here, clear relations among the query images can be used to select the true genuine identity.

Table 8.1: Number of classes for each case

\begin{tabular}{|c|c|c|c|c|c|c|c|}
\hline Threshold & $1-\mathrm{A}$ & $1-\mathrm{B}$ & $2-\mathrm{A}$ & $2-\mathrm{B}$ & $3-\mathrm{A}$ & $3-\mathrm{B}$ & $3-\mathrm{C}$ \\
\hline 0.32 & 252 & 179 & 36240 & 0 & 21 & 0 & 0 \\
\hline
\end{tabular}

Intuitively, if we want to prove that involvement of a social network improves the performance, we need to show that involvement of a social network corrects wrong matches and resolves ambiguities. More specificity, when both genuine and imposter scores are smaller than the threshold, it should be high possibility that genuine one still will be selected does not matter if it is larger or smaller than the imposter one. Here we assume that the imposters are uniformly distributed. It means, for a certain class, the possibilities to be its imposter are same for all other classes. In this chapter, only pairwise relations $\left(P_{1}-P_{2}\right)$ will be analyzed. More complex models can be designed and verified in a similar way. 
Case 2-A $\left(P_{1}\right)$ vs. Case 3 - $\mathbf{A}$ or 3 -B $\left(P_{2}\right)$ In this case, if a class $P_{1}$ can be identified correctly without any ambiguity, then the ambiguity will not be solved only if one of $P_{2}$ 's imposter is also related to $P_{1}$.

Case 2-B or 3-C $\left(P_{1}\right)$ vs. Case 3 -A or $3-\mathrm{B}\left(P_{2}\right)$ If a class $P_{1}$ is identified incorrectly, then the possible error will be caused by small imposter scores which nothing can be done, or one of $P_{2}$ 's imposter also has a relation with wrong $P_{1}$. In this case, proposed method will degrade the performance. However, the possible damage caused by the proposed method is extremely small because of the low possibility.

Case 3-A or 3-B $\left(P_{1}\right)$ vs. Case 3-A or 3-B $\left(P_{2}\right) \quad$ In this case, the additional error will be caused by that one of $P_{1}^{\prime}$ 's imposter $P_{1}^{\prime}$ also has a relation with $P_{2}$ 's imposter $P_{2}^{\prime}$.

As stated earlier, each node of the network will be assigned an iris code. Then the frequency of each scenario can be counted. To evaluate the average performance, node-iriscode mapping was re-arranged 100 times. The frequency of their occurrence are summarized in Table 8.2. When social connections get involved, totally 48,168 ambiguities are correctly solved.

Table 8.2: The average frequencies for different scenarios (threshold: 0.32)

\begin{tabular}{|c|c|c|c|c|c|c|c|}
\hline Case & $1-\mathrm{A}$ & $1-\mathrm{B}$ & $2-\mathrm{A}$ & $2-\mathrm{B}$ & $3-\mathrm{A}$ & $3-\mathrm{B}$ & $3-\mathrm{C}$ \\
\hline $3-\mathrm{A}$ & 0 & 0 & 481.54 & 0 & 0.14 & 0 & 0 \\
\hline $3-\mathrm{B}$ & 0 & 0 & 0 & 0 & 0 & 0 & 0 \\
\hline
\end{tabular}

\section{Verification}

Biometric systems can operate at different security level depending on applications. For example,s credit card application needs a more stringent verification threshold than a checking account balance check. In this chapter, it is assumed that all files in the company were broadly partitioned into 8 classes. Each class has a iris biometric verification threshold to protect the files. Higher security requests a more stringent threshold. A set of well designed thresholds are provided in Table 8.3. 
Table 8.3: Thresholds for different classes

\begin{tabular}{|c|c|c|c|c|c|c|c|c|}
\hline Class & 1 & 2 & 3 & 4 & 5 & 6 & 7 & 8 \\
\hline Threshold & 0.32 & 0.2 & 0.1 & 0.075 & 0.05 & $1 / 40$ & $1 / 80$ & $1 / 160$ \\
\hline
\end{tabular}

Mesh Network To simplify the selection, mesh structure network will be considered first. In a mesh network, all nodes will be treated similarly.In a company, the president of the company has the same security status as all regular employees. When a person $A$ needs a referral to boost his/her verification performance, $A$ can ask his/her colleges or friends for help. The boosting power provided by a referral is determined based on his/her 1) performance and 2) uniqueness. The performance $I_{t h}$ index here is the clearance level which the referral can directly reach. The uniqueness $R$ is evaluated in a closed system using the ordering rank of the claimed genuine score when the query image is compared with all enrolments. The boosting power $P$ will be multiplied with the original matching score, and is calculated using formula (8.1):

$$
P=\alpha^{I_{\text {th }}} * \beta^{1 / R}
$$

where $\alpha$ and $\beta$ are constants. For iris biometrics, $\alpha=0.97$ and $\beta=0.9$.

The performance is tested on a simulated community structure. Any selected person may randomly select friends as referrals. With more and more referrals, how low the adjusted matching score can reach is analyzed and the result is presented in Fig. 8.20.

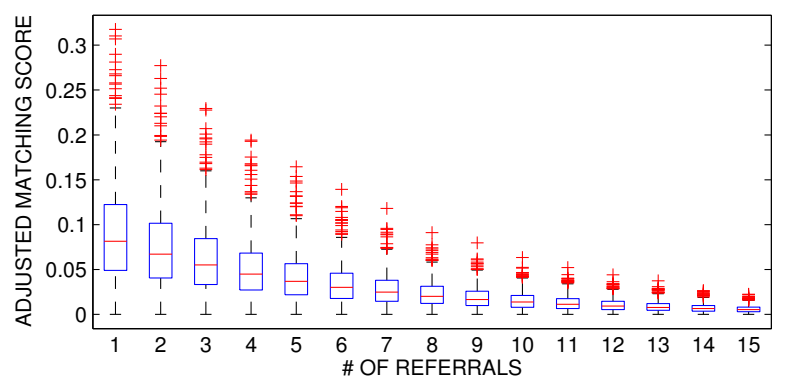

Figure 8.20: The trend of decreasing matching scores when the number of referrals increases.

How many friends they need to reach a certain security level is plotted in Fig. 8.21. 


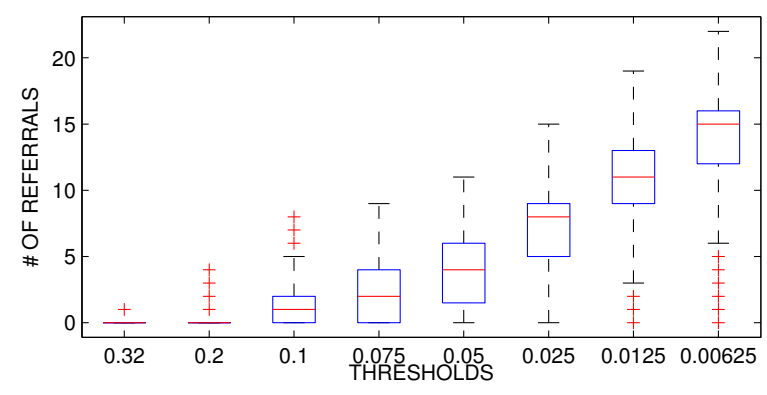

Figure 8.21: The trend of increasing number of referrals when the verification threshold is decreasing.

Hierarchy Network In a 1099 people company, all employees have a grade. Top ranked level E(5) President have two level D(4) Directors. Each Director has four level C(3) Senior Managers. All Senior Managers supervise eight level B(2) Managers. Every 16 level A(1) normal employees form an office, and it is supervised by a Manager. The hierarchical structure is illustrated in Fig. 8.22, and a visualization of the whole network can be found in Fig. 8.23 .

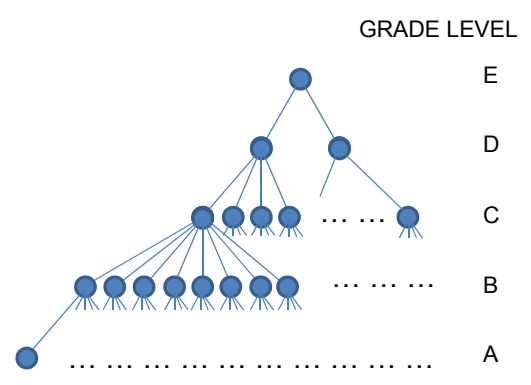

Figure 8.22: The hierarchical structure in the company.

Table 8.4: Number of people at each level

\begin{tabular}{|c|c|c|c|c|c|}
\hline Grade Levels & $\mathrm{E}$ & $\mathrm{D}$ & $\mathrm{C}$ & $\mathrm{B}$ & $\mathrm{A}$ \\
\hline$\#$ & 1 & 2 & $2^{*} 4=8$ & $8^{*} 8=64$ & $64^{*} 16=1024$ \\
\hline
\end{tabular}

When a person A asking for a referral, A's direct supervisor B, and all lower level employees under B's supervision are the only people to ask for referral. The boosting power provided by a referral now is determined based on his/her 1) performance; 2) uniqueness 


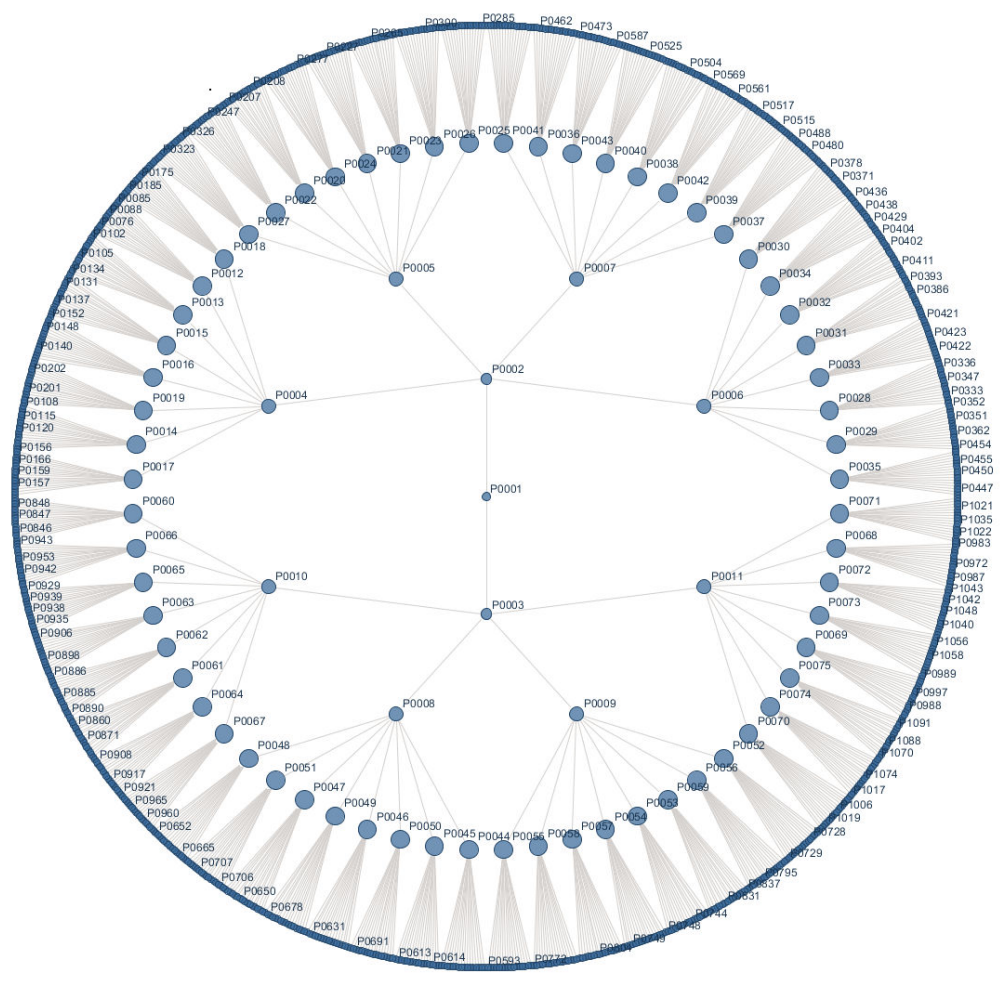

Figure 8.23: The plot of the hierarchy network.

and 3) grade level $G$. The performance and the uniqueness are the same as before. Higher grade level officers will provide larger boosting power because they have a little bit more rank advantage. They can be regarded as having a higher "credit score". Of course the direct supervisor is the best choice when someone is looking for a referral. In this kind of network, the boosting power $P$ is calculated using formula (8.2):

$$
P=\alpha^{I_{t h}} * \beta^{1 / R} * \gamma^{G}
$$

where $\alpha, \beta$ and $\gamma$ are constants. For iris biometrics, $\gamma=0.97$.

The average performance on 1,000 trails for 1,099 classes is summarized in Table 8.5. And for Class 5, the symmetric error bars that are two standard deviation units in length is illustrated in Fig. 8.24. As we can see, Level D achieved the best performance when they are able to find referrals because presidents can not find a higher level referrals than themselves. 
Table 8.5: The average number of referrals needed for each scenario

\begin{tabular}{|c|c|c|c|c|c|}
\hline Class & Level E & Level D & Level C & Level B & Level A \\
\hline 1 & 0.0130 & 0.0100 & 0.0107 & 0.0097 & 0.0100 \\
\hline 2 & 0.1370 & 0.1045 & 0.1206 & 0.1239 & 0.1445 \\
\hline 3 & 0.8250 & 0.7075 & 0.8194 & 0.9513 & 1.2203 \\
\hline 4 & 1.2190 & 1.0820 & 1.2703 & 1.5264 & 2.0345 \\
\hline 5 & 1.9750 & 1.7870 & 2.0871 & 2.5745 & 3.5343 \\
\hline 6 & 3.5160 & 3.2555 & 3.8207 & 4.7015 & $6.6081(0.0 \%)$ \\
\hline 7 & 5.2310 & 4.9835 & 5.8869 & 7.1557 & $9.8393(1.8 \%)$ \\
\hline 8 & 7.0020 & 6.9705 & 8.1457 & 10.0482 & $12.1028(22.6 \%)$ \\
\hline
\end{tabular}

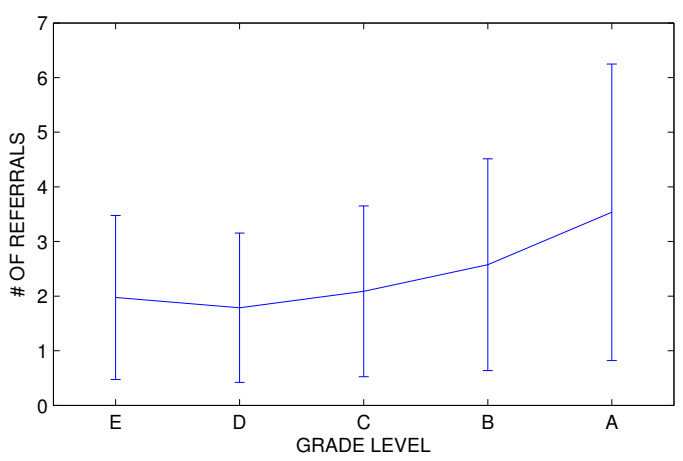

Figure 8.24: The number of referrals needed for each grade level to access class 5 files.

\subsubsection{Discussions}

The social information is used differently in identification and verification scenarios. Identification: FBI (Federal Bureau of Investigation) may already have the social connection information available when they try to identify a fugitive, and they just want a precise identification. Verification: it is not necessary to save the social connection information into the system at anytime.

It is demonstrated that social information can be used to increase the population coverage of the existing biometrics systems. More precise identification performance can be achieved and more complex verification settings can be adopted. Iris biometrics is used to demonstrate the proposed model. 


\section{Chapter 9}

\section{Conclusion}

In this dissertation, various components of iris recognition system are studied in depth. The problems of iris quality evaluation, cross spectral iris recognition, non-ideal iris segmentation, iris encoding, performance prediction and iris image generations are formulated. Novel solutions to these problems are designed, and the performance of developed algorithms is analyzed.

\subsection{Contributions}

The main novelties and contributions of this dissertation are:

- A novel method to generate a large scale realistic database of iris images is developed. It provides an option to compare efficiency, limitations, and capabilities of newly designed iris recognition algorithms through their testing on a large scale data set of generated irises (Ch. 2) [115].

- A cross-spectral iris matching method for comparison of iris images in color range with iris images in Near-Infrared (NIR) range is proposed. Both visual inspection of the predicted image and the verification performance indicate that the adaptive mapping linking NIR image and color image is a potential solution to the problem of matching NIR images vs. color images in practice (Ch. 3) [132].

- A number of iris quality measures are designed to evaluate quality of iris images and iris video frames. This set of newly developed quality measures can be 
used to improve the matching performance of iris recognition systems (Ch. 4) [119].

- A robust quality-based iris segmentation method is developed. It has the capability to reliably segment non-ideal imagery that is simultaneously affected with such factors as specular reflection, blur, lighting variation, occlusion and off-angle of images (Ch. 5) [133].

- An encoding technique using local ordinal information is introduced. It is robust with respect to a number of nonidealities in iris images and it can be flawlessly integrated into the traditional filter-based recognition systems for a better performance (Ch. 6) [59].

- An encoding technique using $2 \mathrm{D}$ median filter is designed. It can handle iris images acquired under non-uniform ambient lighting, and can be used as an useful complementary encoding technique in hierarchical iris recognition systems (Ch. 6) $[134]$.

- An unique "cancelable iris biometric" solution is proposed. Both registrationfree transforms and salting procedures can solve the privacy and security issues at the same time (Ch. 6) [135].

- Three methods to improve the performance of biometric matchers based on vectors of quality measures associated with biometric samples are developed. Significant performance improvements can be achieved from three different ways when a good set of quality measures is available (Ch. 7) [136].

- A method to increase iris capture volume for acquisition of iris on the move from a distance is introduced. This concept can be adopted to applications such as airport surveillance or security check point (Ch. 8).

- A method to improve performance of biometric systems due to available soft data in the form of links and connections in a relevant social network is proposed. It is an alternative and also complimentary approach to involving multiple modalities for very large population coverage applications (Ch. 8). 


\section{References}

[1] Wikipedia, "Iris recognition," http://en.wikipedia.org/wiki/Iris_recognition, accessed December 05, 2011.

[2] Clyde W. Oyster, The Human Eye Structure and Function, Sinauer Associates, 1999.

[3] John Daugman and Cathryn Downing, "Epigenetic randomness, complexity and singularity of human iris patterns," Proceedings of the Royal Society (London): B, vol. 268, no. 1477, pp. 1737-1740, 2001.

[4] John Daugman, "Iris recognition," American Scientist, vol. 89, pp. 326-333, JulyAugust 2001.

[5] Leonard Flom and Aran Safir, "Iris recognition system," United States Patent Application No.: US19850703312 19850220 , February 1987.

[6] James R. Matey, Oleg Naroditsky, Keith Hanna, Ray Kolczynski, Dominick LoIacono, Shakuntala Mangru, Michael Tinker, Thomas Zappia, and Wenyi Y. Zhao, "Iris on the move $^{T M}$ : Acquisition of images for iris recognition in less constrained environments," Proceedings of the IEEE, vol. 94, no. 11, pp. 1936-1947, November 2006.

[7] Hugo Proença and Luís A. Alexandre, "Ubiris: A noisy iris image database," in Proceedings of International Conference on Image Analysis and Processing (ICIAP'05), 2005, vol. 1, pp. 970-977.

[8] John Daugman, "How iris recognition works," IEEE Transactions on Circuits and Systems for Video Technology, vol. 14, no. 1, pp. 21-30, January 2004.

[9] Peng Yao, Jun Li, Xueyi Ye, Zhenquan Zhuang, and Bin Li, "Iris recognition algorithm using modified log-gabor filters," in Proceedings of the 18th International Conference on Pattern Recognition (ICPR'06), Washington, DC, USA, August 2006, vol. 4, pp. 461-464, IEEE Computer Society.

[10] Zhenan Sun, Tieniu Tan, and Yunhong Wang, "Robust encoding of local ordinal measures: A general framework of iris recognition," in Springer LNCS 308\%: Biometric Authentication, 2004, pp. 270-282.

[11] Chenhong Lu and Zhaoyang Lu, "Efficient iris recognition by computing discriminable textons," in International Conference on Neural Networks and Brain, October 2005, vol. 2, pp. 1164-1167. 
[12] Chia-Te Chou, Sheng-Wen Shih, Wen-Shiung Chen, and Victor W. Cheng, "Iris recognition with multi-scale edge-type matching," in Proceedings of the 18th International Conference on Pattern Recognition (ICPR'06), Washington, DC, USA, August 2006, vol. 4, pp. 545-548, IEEE Computer Society.

[13] Li Ma, Tieniu Tan, Yunhong Wang, and Dexin Zhang, "Efficient iris recognition by characterizing key local variations," IEEE Transactions on Image Processing, vol. 13, no. 6, pp. 739-750, June 2004.

[14] Donald Monro, Soumyadip Rakshit, and Dexin Zhang, "Dct-based iris recognition," IEEE Transactions on Pattern Analysis and Machine Intelligence, vol. 29, no. 4, pp. 586-595, April 2007.

[15] Emine Krichen, M. Anouar Mellakh, Sonia Garcia-Salicetti, and Bernadette Dorizzi, "Iris identification using wavelet packets," in Proceedings of the 17th International Conference on Pattern Recognition (ICPR'04), Washington, DC, USA, August 2004, vol. 4, pp. 335-338, IEEE Computer Society.

[16] Florence Rossant, Manuel Torres Eslave, Thomas Ea, Frederic Amiel, and Amara Amara, "Iris identification and robustness evaluation of a wavelet packets based algorithm," in International Conference on Image Processing, September 2005, pp. 2589-2594.

[17] Erik Rydgren, Thomas Ea, Frederic Amiel, Florence Rossant, Amara Amara, and Carmen Sanchez-Reillo, "Iris features extraction using wavelet packets," in International Conference on Image Processing, 2004, pp. II:861-864.

[18] Peeranat Thoonsaengngam, Kittipol Horapong, Somying Thainimit, and Vutipong Areekul, "Efficient iris recognition using adaptive quotient thresholding," in Proceedings of International Conference on Biometrics (ICB'06), January 2006, pp. 472-478.

[19] Onsy Abdel Alim and Maha Sharkas, "Iris recognition using discrete wavelet transform and artificial neural net," in Proceedings of the 46th IEEE International Midwest Symposium on Circuits and Systems (MWSCAS'03), December 2003, vol. 1, pp. 337340.

[20] Jain Jang, Kang Ryoung Park, Jinho Son, and Yillbyung Lee, "A study on multi-unit iris recognition," in International Conference on Control, Automation, Robotics and Vision, December 2004, vol. 2, pp. 1244-1249.

[21] Ya ping Huang, Si wei Luo, and En yi Chen, "An efficient iris recognition system," in International Conference of Machine Learning and Cybernetics, November 2002, vol. 1, pp. 450-454.

[22] Vivekanand Dorairaj, Natalia A. Schmid, and Gamal Fahmy, "Performance evaluation of iris based recognition system implementing PCA and ICA encoding techniques," in SPIE 5779: Biometric Technology for Human Identification II, 2005, vol. 5779, pp. $51-58$. 
[23] Byungjun Son, Hyunsuk Won, Gyundo Kee, and Yillbyung Lee, "Discriminant iris feature and support vector machines for iris recognition," in International Conference on Image Processing, 2004, vol. 2, pp. 865-868.

[24] Chia Te Chu and Ching-Han Chen, "High performance iris recognition based on lda and lpcc," in 17th International Conference on Tools with Artificial Intelligence, 2005, pp. $417-421$.

[25] Ching-Han Chen and Chia Te Chu, "Low complexity iris recognition based on wavelet probabilistic neural networks," in International Joint Conference on Neural Networks, 2005, pp. 1930-1935.

[26] Zhenan Sun, Tieniu Tan, and Xianchao Qiu, "Graph matching iris image blocks with local binary pattern," in Proceedings of International Conference on Biometrics (ICB'06), January 2006, pp. 366-372.

[27] Robert W. Ives, Anthony J. Guidry, and Dolores M. Etter, "Iris recognition using histogram analysis," in Thirty-Eighth Asilomar Conference on Signals, Systems, and Computers, November 2004, pp. I:562-566.

[28] Institute of Automation, Chinese Academy of Sciences, China, "CASIA iris database (ver. 1.0)," http://biometrics.idealtest.org/dbDetailForUser.do?id= 1, accessed December 05, 2011.

[29] Malaysia Multimedia University, "Malaysia multimedia university iris database," http://pesona.mmu.edu.my/ ccteo/, accessed December 05, 2011.

[30] Michal Dobeš and Libor Machala, "UPOL iris database," http://phoenix.inf .upol . cz/iris/, accessed December 05, 2011.

[31] University of Bath, "BATH iris database (free version)," http://www. smartsensors . co.uk/information/bath-iris-image-database/, accessed December 05, 2011.

[32] Anthony J. Mansfield and James L. Wayman, "Best practices in testing and reporting performance of biometric devices," http://www.npl.co.uk/upload/pdf/ biometrics_bestprac_v2_1.pdf, accessed December 05, 2011.

[33] James Wayman, Anil Jain, Davide Maltoni, and Dario Maio, Biometric Systems: Technology, Design, and Performance Evaluation, Springer-Verlag, New York, 1 edition, December 2004.

[34] Jiali Cui, Yunhong Wang, Junzhou Huang, Tieniu Tan, and Zhenan Sun, "An iris image synthesis method based on pca and super-resolution," in Proceedings of the 17th International Conference on Pattern Recognition (ICPR'04), Washington, DC, USA, August 2004, vol. 4, pp. 471-474, IEEE Computer Society.

[35] Sarvesh Makthal and Arun Ross, "Synthesis of iris images using markov random fields," in Proceedings of the 13th European Signal Processing Conference (EUSIPCO'05), Antalya, Turkey, September 2005. 
[36] Aaron Lefohn, Brian Budge, Peter Shirley, Richard Caruso, and Erik Reinhard, "An ocularist's approach to human iris synthesis," IEEE Computer Graphics and Applications, vol. 23, no. 6, pp. 70-75, November / December 2003.

[37] Lakin Wecker, Faramarz Samavati, and Marina Gavrilova, "Iris synthesis: a reverse subdivision application," in Proceedings of the 3rd International Conference on Computer Graphics and Interactive Techniques in Australasia and South East Asia (GRAPHITE'05), Dunedin, New Zealand, November, pp. 121-125.

[38] Miles Research, "Iris pigmentation research info," http://www.milesresearch.com/ iris/, accessed December 05, 2011.

[39] Miles Research, "Iris images from film camera," http://www.milesresearch.com/ download/exampleirisimages.ppt, accessed December 05, 2011.

[40] Farida Sharan, Iridology - a complete guide to diagnosing through the iris and to related forms of treatment, HarperCollins, Hammersmith, London, October 1992.

[41] Libor Masek and Peter Kovesi, "MATLAB source code for a biometric identification system based on iris patterns," 2003.

[42] Anuj Srivastava, "Stochastic models for capturing image variability," IEEE Signal Processing Magazine, vol. 19, no. 5, pp. 63-76, September 2002.

[43] "Brodatz textures database," http://www.ux.uis.no/ tranden/brodatz.html, accessed December 05, 2011.

[44] Manik Varma and Andrew Zisserman, "Filter banks matlab code," http://www. robots.ox.ac.uk/ vgg/research/texclass/filters.html, accessed December 05, 2011.

[45] Libor Masek, "Recognition of human iris patterns for biometric identification," Bachelor's Dissertation, The School of Computer Science and Software Engineering, The University of Western Australia, 2003.

[46] John Daugman, "High confidence visual recognition of persons by a test of statistical independence," IEEE Transactions on Pattern Analysis and Machine Intelligence, vol. 15, no. 11, pp. 1148-1161, November 1993.

[47] The MathWorks, Inc., MATLAB help.

[48] Hugo Proença, Sílvio Filipe, Ricardo Santos, João Oliveira, and Luís A. Alexandre, "The UBIRIS.v2: A database of visible wavelength images captured on-the-move and at-a-distance," IEEE Transactions on Pattern Analysis and Machine Intelligence, vol. 32, no. 8, pp. 1529-1535, August 2010.

[49] MicroImages, Inc., "Introduction to hyperspectral imaging," http://www. microimages.com/getstart/pdf/hyprspec.pdf, accessed December 05, 2011.

[50] James M. Ellis, "Searching for oil seeps and oil-impacted soil with hyperspectral imagery," Earth Observation Magazine, pp. 25-28, January 2001. 
[51] Gary Shaw and Dimitris Manolakis, "Signal processing for hyperspectral image exploitation," IEEE Signal Processing Magazine, vol. 19, no. 1, pp. 12-16, January 2002 .

[52] Zhihong Pan, Glenn Healey, Manish Prasad, and Bruce Tromberg, "Face recognition in hyperspectral images," IEEE Transactions on Pattern Analysis and Machine Intelligence, vol. 25, no. 12, pp. 1552-1560, December 2003.

[53] Robert K. Rowe, Kristin A. Nixon, and Stephen P. Corcoran, "Multispectral fingerprint biometrics," in Information Assurance Workshop, 2005. IAW '05. Proceedings from the Sixth Annual IEEE SMC, 2005, pp. 14-20.

[54] Robert K. Rowe, Umut Uludag, Meltem Demirkus, Sulan Parthasaradhi, and Anil K. Jain, "A multispectral whole-hand biometric authentication system," in Biometrics Symposium, 200\%, September 2007, pp. 1-6.

[55] Mark J. Burge and Matthew K. Monaco, "Multispectral iris fusion for enhancement, interoperability, and cross wavelength matching," 2009, vol. 7334, p. 73341D, SPIE.

[56] Mark J. Burge and Matthew K. Monaco, "Multispectral iris fusion for enhancement and interoperability," United States Patent Application 20090279790, November 2009.

[57] Christopher Boyce, Arun Ross, Matthew Monaco, Lawrence Hornak, and Xin Li, "Multispectral iris analysis: A preliminary study," in Computer Vision and Pattern Recognition Workshop on Biometrics(CVPRW), June 2006, pp. 51-59.

[58] Jinyu Zuo, Nathan D. Kalka, and Natalia A. Schmid, "A robust iris segmentation procedure for unconstrained subject presentation," in Proceedings of 2006 Biometric Consortium Conference (BCC'06), September 2006.

[59] Jinyu Zuo and Natalia A. Schmid, "On a local ordinal binary extension to Gabor wavelet-based encoding for improved iris recognition," in Proceedings of 2007 Biometric Consortium Conference (BCC'07), September 2007.

[60] "Biometric quality workshop, National Institute of Standards and Technology (NIST)," http://www.nist.gov/itl/iad/ig/bio_quality_wkshopi.cfm, March 8-9 2006.

[61] "Biometric quality workshop II, National Institute of Standards and Technology (NIST)," http://www.nist.gov/itl/iad/ig/bio_quality_wkshopii.cfm, November 7-8 2007.

[62] Kevin W. Bowyer, Karen Hollingsworth, and Patrick J. Flynn, "Image understanding for iris biometrics: A survey," Journal of Computer Vision and Image Understabding, vol. 110, pp. 281-307, 2007.

[63] Xiaodong Zhu, Yuanning Liu, Xing Ming, and Qingliang Cui, "A quality evaluation method of iris images sequence based on wavelet coefficients in "region of interest"," in CIT '04: Proceedings of the The Fourth International Conference on Computer and Information Technology, Washington, DC, USA, 2004, pp. 24-27, IEEE Computer Society. 
[64] Yi Chen, Sarat C. Dass, and Anil K. Jain, "Localized iris image quality using 2-D wavelets," in Proceedings of International Conference on Biometrics (ICB'06), January 2006, pp. 373-381.

[65] Li Ma, Tieniu Tan, Yunhong Wang, and Dexin Zhang, "Personal identification based on iris texture analysis," IEEE Transactions on Pattern Analysis and Machine Intelligence, vol. 25, no. 12, pp. 1519-1533, 2003.

[66] GuangHua Zhang and Marcos Salganicoff, "Method of measuring the focus of close-up images of eyes," September 1999.

[67] Byung Jun Kang and Kang Ryoung Park, "A study on iris image restoration," in International Conference on Audio- and Video-Based Biometric Person Authentication, 2005, pp. 31-40.

[68] Craig Belcher and Yingzi Du, "Information distance based selective feature clarity measure for iris recognition," in Proc. of the SPIE Symposium on Defense and Security. Conf. on Human Identification Technology IV., Orlando, FL, April 2007, vol. 6494, pp. 64940 E1-E12.

[69] Nathan D. Kalka, "Image quality assessment for iris biometric," M.S. thesis, West Virginia University, Morgantown, WV, USA, April 2005.

[70] Nathan D. Kalka, Jinyu Zuo, Natalia A. Schmid, and Bojan Cukic, "Image quality assessment for iris biometric," in SPIE 6202: Biometric Technology for Human Identification III, April 2006, vol. 6202, pp. 6202:D1-D11.

[71] National Institute of Standards and Technology (NIST), "Iris challenge evaluation," http://www.nist.gov/itl/iad/ig/ice.cfm, accessed December 05, 2011.

[72] National Institute of Standards and Technology (NIST), "Multiple biometric grand challenge," http://www.nist.gov/itl/iad/ig/mbgc.cfm, accessed December 05, 2011.

[73] Jinyu Zuo and Natalia A. Schmid, "An automatic algorithm for evaluating the precision of iris segmentation," in International Conference on Biometrics: Theory, Applications, and Systems (BTAS'08), September 2008.

[74] Xiaomei Liu, Kevin W. Bowyer, and Patrick J. Flynn, "Experiments with an improved iris segmentation algorithm," in Fourth IEEE Workshop on Automatic Identification Technologies (AutoID), Buffalo, NY, October 2005, pp. 118-123.

[75] Natalia A. Schmid and Francesco Nicolo, "A method for selecting and ranking quality metrics for optimization of biometric recognition systems," in IEEE Computer Society Conference on Computer Vision and Pattern Recognition Workshops (CVPR Workshops 2009) Accepted, June 2009, pp. 1-8.

[76] Center for Biometric Authentication and Testing, Institute of Automation, Chinese Academy of Sciences, China, "CASIA iris database (ver. 3.0)," http://biometrics. idealtest.org/dbDetailForUser.do?id=3, accessed December 05, 2011. 
[77] Arun Ross, Simona Crihalmeanu, Rohin Govindarajan, Lawrence A. Hornak, and Stephanie A. C. Schuckers, "A centralized web-enabled multimodal biometric database," in Proceedings of Biometric Consortium Conference (BCC'04), Crystal City, VA, USA, September 2004.

[78] Center for Identification Technology Research, "WVU off-angle iris database," http: //www.citer.wvu.edu, accessed December 05, 2011.

[79] Theodore A. Camus and Richard P. Wildes, "Reliable and fast eye finding in close-up images," in Proceedings of the 16th International Conference on Pattern Recognition (ICPR'02), Washington, DC, USA, August 2002, vol. 1, pp. 389-394, IEEE Computer Society.

[80] Hugo Proença and Luís A. Alexandre, "Iris segmentation methodology for noncooperative recognition," vol. 153, no. 2, pp. 199-205, April 2006.

[81] Richard P. Wildes, "Iris recognition: An emerging biometric technology," Proceedings of the IEEE, vol. 85, no. 9, pp. 1348-1363, September 1997.

[82] Qi-Chuan Tian, Quan Pan, Yong-Mei Cheng, and Quan-Xue Gao, "Fast algorithm and application of Hough transform in iris segmentation," in Proceedings of the 3rd International Conference on Machine Learning and Cybernetics, August 2004, vol. 7, pp. 3977-3980.

[83] Craig Fancourt, Luca Bogoni, Keith Hanna, Yanlin Guo, Richard Wildes, Naomi Takahashi, and Uday Jain, "Iris recognition at a distance," in Proceedings of International Conference on Audio- and Video-Based Biometric Person Authentication (AVBPA'05), 2005, pp. 1-13.

[84] Joaquim de Mira Jr. and Joceli Mayer, "Image feature extraction for application of biometric identification of iris - a morphological approach," in Proceedings of XVI Brazilian symposium on Computer Graphics and Image Processing (SIBGRAPI'03), October 2003, pp. 391-398.

[85] Eric Sung, Xilin Chen, Jie Zhu, and Jie Yang, "Towards non-cooperative iris recognition systems," in Proceedings of the 7th International Conference on Control, Automation, Robotics and Vision (ICARCV'02), December 2002, vol. 2, pp. 990-995.

[86] Jinyu Zuo, Nalini K. Ratha, and Jonathan H. Connell, "A new approach for iris segmentation," in IEEE Computer Society Conference on Computer Vision and Pattern Recognition Workshops (CVPR Workshops 2008), June 2008, pp. 1-6.

[87] Aditya Abhyankar, Lawrence Hornak, and Stephanie Schuckers, "Off-angle iris recognition using bi-orthogonal wavelet network system," in Proceedings of the 4th IEEE Workshop on Automatic Identification Technologies (AutoID), Buffalo, NY, October 2005, pp. 239-244.

[88] Niladri B. Puhan, Natarajan Sudha, and Xudong Jiang, "Robust eyeball segmentation in noisy iris images using Fourier spectral density," in 6th International Conference on Information, Communications and Signal Processing, December 2007, pp. 1-5. 
[89] Shrinivas Pundlik, Damon L. Woodard, and Stanley T. Birchfield, "Non-ideal iris segmentation using graph cuts," in IEEE Computer Society Conference on Computer Vision and Pattern Recognition Workshops (CVPR Workshops 2008), January 2008, pp. 1-6.

[90] Amjad Zaim, Mahmoud K. Quweider, Jeff Scargle, Juan Iglesias, and Lappoon R. Tang, "A robust and accurate segmentation of iris images using optimal partitioning," in Proceedings of the 18th International Conference on Pattern Recognition (ICPR'06), Washington, DC, USA, August 2006, vol. 4, pp. 578-581, IEEE Computer Society.

[91] Wai-Kin Kong and David Zhang, "Detecting eyelash and reflection for accurate iris segmentation," International Journal of Pattern Recognition and Artificial Intelligence, vol. 17, no. 6, pp. 1025-1034, 2003.

[92] XiaoFu He and PengFei Shi, "A novel iris segmentation method for hand-held capture device," in Proceedings of International Conference on Biometrics (ICB'06), January 2006, pp. 479-485.

[93] Junzhou Huang, Yunhong Wang, Jiali Cui, and Tieniu Tan, "Noise removal and impainting model for iris image," in International Conference on Image Processing, October 2004, pp. 2:869-872.

[94] John Daugman, "Probing the uniqueness and randomness of iriscodes: Results from 200 billion iris pair comparisons," Proceedings of the IEEE, vol. 94, no. 11, pp. 19271935, November 2006.

[95] John Daugman, "New methods in iris recognition," IEEE Transactions on Systems, Man, and Cybernetics, Part B, vol. 37, no. 5, pp. 1167-1175, September 2007.

[96] Glenn Shafer, A Mathematical Theory of Evidence, Princeton University Press, Princeton, NJ, 1976.

[97] Tony F. Chan, Jianhong Shen, and Luminita Vese, "Variational PDE models in image processing," in Notices of the American Mathematical Society, January 2003, vol. 50, pp. $14-26$.

[98] Robert J. Serfling, Approximation Theorems of Mathematical Statistics, WileyInterscience, 1980.

[99] Jae S. Lim, Two-Dimensional Signal and Image Processing, Prentice Hall, Englewood Cliffs, NJ, 1990.

[100] Purva Burve and Xin Li, "Robust preprocessing techniques for nonideal iris recognition," in Proceedings of Biometric Consortium Conference 2005 (BCC'05), Virginia, USA, September 2005.

[101] Haitao Wang, Stan Z. Li, and Yangsheng Wang, "Face recognition under varying lighting conditions using self quotient image," in Proceedings of the 6th IEEE International Conference on Automatic Face and Gesture Recognition (FGR'04), May 2004, pp. 819-824. 
[102] Amnon Shashua and Tammy Riklin-Raviv, "The quotient image: class-based rerendering and recognition with varying illuminations," Trans. on Pattern Analysis and Machine Intelligence, vol. 23, no. 2, pp. 129-139, 2001.

[103] Tammy Riklin-Raviv and Amnon Shashua., "The quotient image: class based recognition and synthesis under varying illumination," in Proceedings of Conference on Computer Vision and Pattern Recognition, 1999, pp. 566-571.

[104] E. P. Simoncelli, "matlabPyrTools," http://www.cns.nyu.edu/ lcv/software.php, accessed December 05, 2011.

[105] Timo Ojala, Matti Pietikäinen, and Topi Mäenpää, "A generalized local binary pattern operator for multiresolution gray scale and rotation invariant texture classification," in Proceedings of Second International Conference on Advances in Pattern Recognition, Rio de Janeiro, Brazil, 2001, pp. 397-406.

[106] Timo Ojala, Matti Pietikäinen, and Topi Mäenpää, "Multiresolution gray-scale and rotation invariant texture classification with local binary patterns," IEEE Transactions on Pattern Analysis and Machine Intelligence, vol. 24, no. 7, pp. 971-987, July 2002.

[107] Andreas E. Savakis and H. Joel Trussell, "Blur identification by residual spectral matching," IEEE Transactions on Image Processing, vol. 2, no. 2, pp. 141-151, Apr 1993.

[108] Nalini K. Ratha, Jonathan H. Connell, and Ruud M. Bolle, "Enhancing security and privacy in biometrics-based authentication systems," IBM Systems Journal, vol. 40, no. 3, pp. 614-634, 2001.

[109] Nalini K. Ratha, Sharat Chikkerur, Jonathan H. Connell, and Ruud M. Bolle, "Generating cancelable fingerprint templates," IEEE Transactions on Pattern Analysis and Machine Intelligence, vol. 29, no. 4, pp. 561-572, 2007.

[110] George I. Davida, Yair Frankel, and Brian J. Matt, "On enabling secure applications through off-line biometric identification," in IEEE Symposium on Security and Privacy, 1998, pp. 148-157.

[111] Ari Juels and Martin Wattenberg, "A fuzzy commitment scheme," in ACM Conference on Computer and Communications Security, April 1999, pp. 28-36.

[112] Siew Chin Chong, Andrew Beng Jin Teoh, and David Chek Ling Ngo, "Iris authentication using privatized advanced correlation filter," in Proceedings of International Conference on Biometrics (ICB'06), January 2006, pp. 382-388.

[113] Siew Chin Chong, Andrew Beng Jin Teoh, and David Chek Ling Ngo, "High security iris verification system based on random secret integration," vol. 102, no. 2, pp. 169$177,2006$.

[114] Feng Hao, Ross Anderson, and John Daugman, "Combining crypto with biometrics effectively," IEEE Transactions on Computers, vol. 55, no. 9, pp. 1081-1088, September 2006 . 
[115] Jinyu Zuo, Natalia A. Schmid, and Xiaohan Chen, "On generation and analysis of synthetic iris images," IEEE Transactions on Information Forensics and Security, vol. 2, no. 1, pp. 77-90, March 2007.

[116] Krzysztof Kryszczuk and Andrzej Drygajlo, "Improving classification with classindependent quality measures: Q-stack in face verification," in Proc. of ICB 200\%, 2007, pp. 1124-1133.

[117] Karthik Nandakumar, Yi Chen, Sarat C. Dass, and Anil K. Jain, "Likelihood ratiobased biometric score fusion," IEEE Trans. on PAMI, vol. 30, no. 2, pp. 342-347, 2008.

[118] Hartwig Fronthaler, Klaus Kollreider, Josef Bigun, Julian Fierrez-Aguilar, Fernando Alonso-Fernandez, Javier Ortega-Garcia, and Joaquin Gonzalez-Rodriguez, "Fingerprint image-quality estimation and its application to multialgorithm verification," IEEE Trans. on IFS, vol. 3, no. 2, pp. 331-338, 2008.

[119] Jinyu Zuo and Natalia A. Schmid, "Global and local quality measures for NIR iris video," in Proc. of the 2009 IEEE CVPR Workshops, Miami, FL, June 2009, pp. 1-6.

[120] Frederick W. Wheeler, A. G. Amitha Perera, Gil Abramovich, Bing Yu, and Peter H. $\mathrm{Tu}$, "Stand-off iris recognition system," in 2nd IEEE International Conference on Biometrics: Theory, Applications and Systems (BTAS 2008), September 2008, pp. $1-7$.

[121] Point Grey Research, Inc., "Products and services: Stereo vision products," http: //www.ptgrey.com/products/stereo.asp, accessed December 05, 2011.

[122] Microsoft, "Introducing Kinect for Xbox 360," http://www .xbox.com/en-US/kinect, accessed December 05, 2011.

[123] PrimeSense Ltd., "What is the primesensor ${ }^{T M}$ depth acquisition technology?," http: //primesense.360.co.il/?p=535, accessed December 05, 2011.

[124] Sebastien Paris, "Face detection toolbox," http://www.mathworks.com/ matlabcentral/fileexchange/24092-face-detection-toolbox, accessed December 05, 2011.

[125] Don Riley, "3D Puma robot demo," http://www.mathworks.com/matlabcentral/ fileexchange/14932-3d-puma-robot-demo, accessed December 05, 2011.

[126] Arun A. Ross, Karthik Nandakumar, and Anil K. Jain, Handbook of Multibiometrics (International Series on Biometrics), Springer-Verlag New York, Inc., Secaucus, NJ, USA, 2006.

[127] Anil K. Jain and Arun Ross, "Multibiometric systems," Commun. ACM, vol. 47, pp. 34-40, January 2004.

[128] Arun Ross, "An introduction to multibiometrics," in Proceeding of the 15th European Signal Processing Conference. (EUSIPCO), Poznan, Poland, September 2007, pp. 1-6. 
[129] Arvind Narayanan and Vitaly Shmatikov, "De-anonymizing social networks," Security and Privacy, IEEE Symposium on, vol. 0, pp. 173-187, 2009.

[130] David Petrou, Andrew Rabinovich, and Hartwig Adam, "Facial recognition with social network aiding," International Patent Application No.: PCT/US2010/044771, June 2010 .

[131] Jure Leskovec, Jon Kleinberg, and Christos Faloutsos, "Graphs over time: densification laws, shrinking diameters and possible explanations," in Proceedings of the eleventh ACM SIGKDD international conference on Knowledge discovery in data mining, New York, NY, USA, 2005, KDD '05, pp. 177-187, ACM.

[132] Jinyu Zuo, Francesco Nicolo, and Natalia A. Schmid, "Cross spectral iris matching based on predictive image mapping," in 2010 Fourth IEEE International Conference on Biometrics: Theory Applications and Systems (BTAS), September 2010, pp. 1-5.

[133] Jinyu Zuo and Natalia A. Schmid, "On a methodology for robust segmentation of nonideal iris images," IEEE Transactions on Systems, Man, and Cybernetics, Part B: Cybernetics, vol. 40, no. 3, pp. 703-718, June 2010.

[134] Jinyu Zuo, Nalini K. Ratha, and Jonathan H. Connell, "Median filter based iris encoding technique," in International Conference on Biometrics: Theory, Applications, and Systems (BTAS'08), September 2008.

[135] Jinyu Zuo, Nalini K. Ratha, and Jonathan H. Connell, "Cancelable iris biometric," in Proceedings of the 19th International Conference on Pattern Recognition (ICPR'08), December 2008.

[136] Jinyu Zuo, Francesco Nicolo, Natalia A. Schmid, and Harry Wechsler, "Adaptive biometric authentication using nonlinear mappings on quality measures and verification scores," in 2010 17th IEEE International Conference on Image Processing (ICIP), September 2010, pp. 4077-4080.

Note: The author has received permission from IEEE to include articles published by the author in IEEE publications (IEEE retains copyright of the published articles included in this dissertation). 